\title{
On Low-Dimensional Solvmanifolds
}

\author{
Christoph Bock
}

MSC 2000: Primary 53C30, 57T15; Secondary 57R17

\section{Introduction}

In this note we want to study compact homogeneous spaces $G / \Gamma$, where $G$ is a connected and simply-connected Lie group and $\Gamma$ a discrete subgroup in $G$. It is well known that the existence of such a $\Gamma$ implies the unimodularity of the Lie group $G$. Recall that a Lie group $G$ is called unimodular if for all $X \in \mathfrak{g}$ holds $\operatorname{tr}$ ad $X=0$, where $\mathfrak{g}$ denotes the Lie algebra of $G$.

If we further demand $G / \Gamma$ to be symplectic (when $G$ is even-dimensional), a result of Chu [11] shows that $G$ has to be solvable.

Therefore, we regard compact quotients of connected and simply-connected solvable Lie groups by discrete subgroups, so called solvmanifolds.

First, we recall the definition of nilpotent and solvable groups resp. Lie algebras.

(i) Let $G$ be group and denote its neutral element by $e$. We define the $d e$ rived series $\left(D^{(k)} G\right)_{k \in \mathbb{N}}$, descending series $\left(G^{(k)}\right)_{k \in \mathbb{N}}$ and ascending series $\left(G_{(k)}\right)_{k \in \mathbb{N}}$ of subgroups in $G$ inductively as follows:

$$
\begin{gathered}
D^{(0)} G:=G^{(0)}:=G, \\
D^{(k)} G:=\left[D^{(k-1)} G, D^{(k-1)} G\right], \quad G^{(k)}:=\left[G, G^{(k-1)}\right], \\
G_{(0)}=\{e\}, \quad G_{(k)}:=\left\{g \in G \mid[g, G] \subset G_{(k-1)}\right\} .
\end{gathered}
$$

$G$ is called nilpotent if there exists $k_{0} \in \mathbb{N}$ such that $G^{\left(k_{0}\right)}=\{e\}$.

$G$ is called solvable if there exists $k_{0} \in \mathbb{N}$ such that $D^{\left(k_{0}\right)} G=\{e\}$.

(ii) Given a Lie algebra $\mathfrak{g}$, one defines the derived, descending and ascending series of subalgebras in $\mathfrak{g}$ via

$$
\begin{gathered}
D^{(0)} \mathfrak{g}:=\mathfrak{g}^{(0)}:=\mathfrak{g} \\
D^{(k)} \mathfrak{g}:=\left[D^{(k-1)} \mathfrak{g}, D^{(k-1)} \mathfrak{g}\right], \quad \mathfrak{g}^{(k)}:=\left[\mathfrak{g}, \mathfrak{g}^{(k-1)}\right] \\
\mathfrak{g}_{(0)}=\{0\}, \quad \mathfrak{g}_{(k)}:=\left\{X \in \mathfrak{g} \mid[X, \mathfrak{g}] \subset \mathfrak{g}_{(k-1)}\right\}
\end{gathered}
$$


and calls $\mathfrak{g}$ nilpotent resp. solvable if its ascending resp. derived series becomes trivial for $k_{0}$ large enough.

We collect some properties in the following proposition. The parts which are not obvious can be found in [59, Section 3.18].

\section{Proposition 1.0.1.}

(i) The subgroups arising in the derived, descending and ascending series of a group are normal. Moreover, they are closed and simply-connected Lie subgroups in the case of a connected and simply-connected Lie group.

(ii) The subalgebras arising in the derived, descending and ascending series of a Lie algebra are ideals.

(iii) A Lie group is nilpotent resp. solvable if and only if its Lie algebra is nilpotent resp. solvable.

We shall examine whether certain solvmanifolds are formal, symplectic, Kähler or Lefschetz. Therefore, we give a brief introduction of these notions.

\section{$1.1 \quad$ Formality}

A differential graded algebra $(D G A)$ is a graded $\mathbb{R}$-algebra $A=\bigoplus_{i \in \mathbb{N}} A^{i}$ together with an $\mathbb{R}$-linear map $d: A \rightarrow A$ such that $d\left(A^{i}\right) \subset A^{i+1}$ and the following conditions are satisfied:

(i) The $\mathbb{R}$-algebra structure of $A$ is given by an inclusion $\mathbb{R} \hookrightarrow A^{0}$.

(ii) The multiplication is graded commutative, i.e. for $a \in A^{i}$ and $b \in A^{j}$ one has $a \cdot b=(-1)^{i \cdot j} b \cdot a \in A^{i+j}$.

(iii) The Leibniz rule holds: $\forall_{a \in A^{i}} \forall_{b \in A} d(a \cdot b)=d(a) \cdot b+(-1)^{i} a \cdot d(b)$

(iv) The map $d$ is a differential, i.e. $d^{2}=0$.

Further, we define $|a|:=i$ for $a \in A^{i}$.

The $i$-th cohomology of a $D G A(A, d)$ is the algebra

$$
H^{i}(A, d):=\frac{\operatorname{ker}\left(d: A^{i} \rightarrow A^{i+1}\right)}{\operatorname{im}\left(d: A^{i-1} \rightarrow A^{i}\right)} .
$$

If $\left(B, d_{B}\right)$ is another DGA, then a $\mathbb{R}$-linear map $f: A \rightarrow B$ is called morphism if $f\left(A^{i}\right) \subset B^{i}, f$ is multiplicative, and $d_{B} \circ f=f \circ d_{A}$. Obviously, any such $f$ induces a homomorphism $f^{*}: H^{*}\left(A, d_{A}\right) \rightarrow H^{*}\left(B, d_{B}\right)$. A morphism of differential graded algebras inducing an isomorphism on cohomology is called quasi-isomorphism. 
Definition 1.1.1. A DGA $(\mathcal{M}, d)$ is said to be minimal if

(i) there is a graded vector space $V=\left(\bigoplus_{i \in \mathbb{N}_{+}} V^{i}\right)=\operatorname{Span}\left\{a_{k} \mid k \in I\right\}$ with homogeneous elements $a_{k}$, which we call the generators,

(ii) $\mathcal{M}=\bigwedge V$,

(iii) the index set $I$ is well ordered, such that $k<l \Rightarrow\left|a_{k}\right| \leq\left|a_{l}\right|$ and the expression for $d a_{k}$ contains only generators $a_{l}$ with $l<k$.

We shall say that $(\mathcal{M}, d)$ is a minimal model for a differential graded algebra $\left(A, d_{A}\right)$ if $(\mathcal{M}, d)$ is minimal and there is a quasi-isomorphism of DGAs $\rho:(\mathcal{M}, d) \rightarrow\left(A, d_{A}\right)$, i.e. it induces an isomorphism $\rho^{*}: H^{*}(\mathcal{M}, d) \rightarrow H^{*}\left(A, d_{A}\right)$ on cohomology.

The importance of minimal models is reflected by the following theorem, which is taken from Sullivan's work [55, Section 5].

Theorem 1.1.2. A differential graded algebra $\left(A, d_{A}\right)$ with $H^{0}\left(A, d_{A}\right)=\mathbb{R}$ possesses a minimal model. It is unique up to isomorphism of differential graded algebras.

We quote the existence-part of Sullivan's proof, which gives an explicit construction of the minimal model. Whenever we are going to construct such a model for a given algebra in this article, we will do it as we do it in this proof.

Proof of the existence. We need the following algebraic operations to "add" resp. "kill" cohomology.

Let $(\mathcal{M}, d)$ be a DGA. We "add" cohomology by choosing a new generator $x$ and setting

$$
\widetilde{\mathcal{M}}:=\mathcal{M} \otimes \bigwedge(x),\left.\quad \tilde{d}\right|_{\mathcal{M}}=d, \quad \tilde{d}(x)=0,
$$

and "kill" a cohomology class $[z] \in H^{k}(\mathcal{M}, d)$ by choosing a new generator $y$ of degree $k-1$ and setting

$$
\widetilde{\mathcal{M}}:=\mathcal{M} \otimes \bigwedge(y),\left.\quad \tilde{d}\right|_{\mathcal{M}}=d, \quad \tilde{d}(y)=z .
$$

Note that $z$ is a polynomial in the generators of $\mathcal{M}$.

Now, let $\left(A, d_{A}\right)$ a DGA with $H^{0}\left(A, d_{A}\right)=\mathbb{R}$. We set $\mathcal{M}_{0}:=\mathbb{R}, d_{0}:=0$ and $\rho_{0}(x)=x$.

Suppose now $\rho_{k}:\left(\mathcal{M}_{k}, d_{k}\right) \rightarrow\left(A, d_{A}\right)$ has been constructed so that $\rho_{k}$ induces isomorphisms on cohomology in degrees $\leq k$ and a monomorphism in degree $(k+1)$.

"Add" cohomology in degree $(k+1)$ to get morphism of differential graded algebras $\rho_{(k+1), 0}:\left(\mathcal{M}_{(k+1), 0}, d_{(k+1), 0}\right) \rightarrow\left(A, d_{A}\right)$ which induces an isomorphism $\rho_{(k+1), 0}^{*}$ on cohomology in degrees $\leq(k+1)$. Now, we want to make the induced map $\rho_{(k+1), 0}^{*}$ injective on cohomology in degree $(k+2)$. 
We "kill" the kernel on cohomology in degree $(k+2)$ (by non-closed generators of degree $(\mathrm{k}+1))$ and define $\rho_{(k+1), 1}:\left(\mathcal{M}_{(k+1), 1}, d_{(k+1), 1}\right) \rightarrow\left(A, d_{A}\right)$ accordingly. If there are generators of degree one in $\left(\mathcal{M}_{(k+1), 0}, d_{(k+1), 0}\right)$ it is possible that this killing process generates new kernel on cohomology in degree $(k+2)$. Therefore, we may have to "kill" the kernel in degree $(k+2)$ repeatedly.

We end up with a morphism $\rho_{(k+1), \infty}:\left(\mathcal{M}_{(k+1), \infty}, d_{(k+1), \infty}\right) \rightarrow\left(A, d_{A}\right)$ which induces isomorphisms on cohomology in degrees $\leq(k+1)$ and a monomorphism in degree $(k+2)$. Set $\rho_{k+1}:=\rho_{(k+1), \infty}$ and $\left(\mathcal{M}_{k+1}, d_{k+1}\right):=\left(\mathcal{M}_{(k+1), \infty}, d_{(k+1), \infty}\right)$.

Inductively we get the minimal model $\rho:(\mathcal{M}, d) \rightarrow\left(A, d_{A}\right)$.

A minimal model $\left(\mathcal{M}_{M}, d\right)$ of a connected smooth manifold $M$ is a minimal model for the de Rahm complex $(\Omega(M), d)$ of differential forms on $M$. The last theorem implies that every connected smooth manifold possesses a minimal model which is unique up to isomorphism of differential graded algebras.

Now, we are able to introduce the notion of formality. Endowed with the trivial differential, the cohomology of a minimal DGA is a DGA itself, and therefore it also possesses a minimal model. In general, this two minimal models need not to be isomorphic.

A minimal differential graded algebra $(\mathcal{M}, d)$ is called formal if there is a morphism of differential graded algebras

$$
\psi:(\mathcal{M}, d) \rightarrow\left(H^{*}(\mathcal{M}, d), d_{H}=0\right)
$$

that induces the identity on cohomology.

This means that $(\mathcal{M}, d)$ and $\left(H^{*}(\mathcal{M}, d), d_{H}=0\right)$ share their minimal model. The following theorem gives an equivalent characterisation.

Theorem 1.1.3 ([48, Theorem 1.3.1]). A minimal model $(\mathcal{M}, d)$ is formal if and only if we can write $\mathcal{M}=\bigwedge V$ and the space $V$ decomposes as a direct sum $V=C \oplus N$ with $d(C)=0, d$ is injective on $N$, and such that every closed element in the ideal $I(N)$ generated by $N$ in $\bigwedge V$ is exact.

This allows us to give a weaker version of the notion of formality.

Definition 1.1.4. A minimal model $(\mathcal{M}, d)$ is called $s$-formal, $s \in \mathbb{N}$, if we can write $\mathcal{M}=\bigwedge V$ and for each $i \leq s$ the space $V^{i}$ generated by generators of degree $i$ decomposes as a direct sum $V^{i}=C^{i} \oplus N^{i}$ with $d\left(C^{i}\right)=0, d$ is injective on $N^{i}$ and such that every closed element in the ideal $I\left(\bigoplus_{i \leq s} N^{i}\right)$ generated by $\bigoplus_{i \leq s} N^{i}$ in $\bigwedge\left(\bigoplus_{i \leq s} V^{i}\right)$ is exact in $\bigwedge V$.

Obviously, formality implies $s$-formality for every $s$.

The following theorem is an immediate consequence of the last definition. 
Theorem 1.1.5. Let $(\mathcal{M}, d)$ be a minimal model, where $\mathcal{M}=\bigwedge V, V=C \oplus N$ with $d(C)=0$ and $d$ is injective on $N$.

Assume that there exist $r, s \in \mathbb{N}_{+}, n \in N^{r}$ and $x \in \bigwedge\left(\bigoplus_{i \leq s} V^{i}\right)$ such that holds

$$
\forall_{c \in C^{r}}(n+c) x \text { is closed and not exact. }
$$

Then $(\mathcal{M}, d)$ is not $\max \{r, s\}$-formal.

A connected smooth manifold is called formal (resp. $s$-formal) if its minimal model is formal (resp. $s$-formal).

The next theorem shows the reason for defining $s$-formality: in certain cases $s$-formality is sufficient for a manifold to be formal.

Theorem 1.1.6 ([21, Theorem 3.1]). Let $M$ be a connected and orientable compact smooth manifold of dimension $2 n$ or $(2 n-1)$.

Then $M$ is formal if and only if it is $(n-1)$-formal.

Example ([21, Corollary 3.3]).

(i) Every connected and simply-connected compact smooth manifold is 2-formal.

(ii) Every connected and simply-connected compact smooth manifold of dimension seven or eight is formal if and only if it is 3-formal.

Proposition 1.1.7 ([21, Lemma 2.11]). Let $M_{1}, M_{2}$ be connected smooth manifolds. They are both formal (resp. s-formal) if and only if $M_{1} \times M_{2}$ is formal (resp. s-formal).

An important tool for detecting non-formality is the concept of Massey products: As we shall see below, the triviality of the Massey products is necessary for formality.

Let $(A, d)$ be a differential graded algebra and $a_{i} \in H^{p_{i}}(A), p_{i}>0,1 \leq i \leq 3$, satisfying $a_{j} \cdot a_{j+1}=0$ for $j=1,2$. Take elements $\alpha_{i}$ of $A$ with $a_{i}=\left[\alpha_{i}\right]$ and write $\alpha_{j} \cdot \alpha_{j+1}=d \xi_{j, j+1}$ for $j=1,2$. The (triple-)Massey product $\left\langle a_{1}, a_{2}, a_{3}\right\rangle$ of the classes $a_{i}$ is defined as

$$
\left[\alpha_{1} \cdot \xi_{2,3}+(-1)^{p_{1}+1} \xi_{1,2} \cdot \alpha_{3}\right] \in \frac{H^{p_{1}+p_{2}+p_{3}-1}(A)}{a_{1} \cdot H^{p_{2}+p_{3}-1}(A)+H^{p_{1}+p_{2}-1}(A) \cdot a_{3}} .
$$

Remark. The definition of the triple-Massey product as an element of a quotient space is well defined, see e.g. [48, Section 1.6].

The next lemma shows the relation between formality and Massey products.

Lemma 1.1.8 ([48, Theorem 1.6.5]). For any formal minimal differential graded algebra all Massey products vanish. 
Corollary 1.1.9. If the de Rahm complex $(\Omega(M), d)$ of a smooth manifold $M$ possesses a non-vanishing Massey product, then $M$ is not formal.

Fernández and Muñoz considered in [22] the geography of non-formal compact manifolds. This means they examined whether there are non-formal compact manifolds of a given dimension with a given first Betti number. They obtained the following theorem:

Theorem 1.1.10. Given $m \in \mathbb{N}_{+}$and $b \in \mathbb{N}$, there are compact oriented $m$ dimensional smooth manifolds with $b_{1}=b$ which are non-formal if and only if one of the following conditions holds:

(i) $m \geq 3$ and $b \geq 2$,

(ii) $m \geq 5$ and $b=1$,

(iii) $m \geq 7$ and $b=0$.

\subsection{Symplectic, Kähler and Lefschetz manifolds}

The main examples of formal spaces are Kähler manifolds. By definition, a Kähler manifold possesses a Riemannian, a symplectic and a complex structure that are compatible in a sense we are going to explain now.

Recall that a symplectic manifold is a pair $(M, \omega)$, where $M$ is a $(2 n)$-dimensional smooth manifold and $\omega \in \Omega^{2}(M)$ is a closed 2-form on $M$ such that $\omega$ is non-degenerate, i.e. $w_{p}^{n} \neq 0$ for all $p \in M$.

\section{Definition 1.2.1.}

(i) An almost complex structure on an even-dimensional smooth manifold $M$ is a complex structure $J$ on the tangent bundle $T M$.

(ii) Let $M, J$ be as in (i) and $\omega \in \Omega^{2}(M)$ a non-degenerate 2-form on M. The 2 -form $\omega$ is called compatible with $J$ if the bilinear form $\langle\ldots, \ldots\rangle$ given by

$$
\forall_{p \in M} \forall_{v, w \in T_{p} M}\langle v, w\rangle=\omega(v, J w)
$$

defines a Riemannian metric on $M$.

(iii) An almost complex structure $J$ on $M$ as in (i) is called integrable if there exists an atlas $\mathcal{A}_{M}$ on $M$ such that

$$
\forall_{u \in \mathcal{A}_{M}} \forall_{p \in \operatorname{Range}(u)} \quad d_{p} u \circ J_{p}=J_{0} \circ d_{p} u: T_{p} M \rightarrow \mathbb{R}^{2 n},
$$

where

$$
J_{0}=\left(\begin{array}{cc}
0 & -I d \\
I d & 0
\end{array}\right) .
$$

$J$ is called complex structure for $M$. 
(iv) A Kähler manifold is a symplectic manifold $(M, \omega)$ with a complex structure $J$ on M such that $\omega$ is compatible with $J$.

If one wants to show that a given almost complex structure is not integrable, it may be hard to disprove the condition (iii) of the last definition. But in [45], Newlander and Nirenberg proved their famous result that an almost complex structure $J$ on a smooth manifold $M$ is integrable if and only if $N_{J} \equiv 0$, where the Nijenhuis tensor $N_{J}$ is defined as

$$
N_{J}(X, Y)=[J X, J Y]-J[J X, Y]-J[X, J Y]-[X, Y]
$$

for all vector fields $X, Y$ on $M$.

For a time, it was not clear whether every symplectic manifold was not in fact Kählerian. Meanwhile, many examples of non-Kählerian symplectic manifolds are known. The first such was given by Thurston in 1976 - the so-called KodairaThurston manifold, see [56].

The difficulty to prove non-existence of any Kähler structure is obvious. Nowadays, two easily verifiable necessary conditions for Kähler manifolds are known. First, we have the main theorem from the work [14] of Deligne, Griffiths, Morgan and Sullivan.

Theorem 1.2.2 ([14, p. 270]). Compact Kähler manifolds are formal.

In order to prove that his manifold is not Kählerian, Thurston used another method. His manifold has first Betti number equal to three and the Hodge decomposition for Kähler manifolds implies that its odd degree Betti numbers have to be even, see e.g. [27, pp. 116 and 117]. This is even satisfied for every Hard Lefschetz manifold.

We say that a symplectic manifold $\left(M^{2 n}, \omega\right)$ is Lefschetz if the homomorphism

$$
\begin{aligned}
& L^{k}: \quad H^{n-k}(M, \mathbb{R}) \quad \longrightarrow H^{n+k}(M, \mathbb{R}) \\
& {[\alpha] \quad \longmapsto \quad\left[\alpha \wedge \omega^{k}\right]}
\end{aligned}
$$

is surjective for $k=n-1$. If $L^{k}$ is surjective for $k \in\{0, \ldots, n-1\}$, then $(M, \omega)$ is called Hard Lefschetz.

Note that for compact $M$ the surjectivity of $L^{k}$ implies its injectivity.

Obviously, the Lefschetz property depends on the choice of the symplectic form. It may be possible that a smooth manifold $M$ possesses two symplectic forms $\omega_{1}, \omega_{2}$ such that $\left(M, \omega_{1}\right)$ is Lefschetz and $\left(M, \omega_{2}\right)$ not. But as mentioned above, the existence of such an $\omega_{1}$ has the following consequence that is purely topological.

Theorem 1.2.3. The odd degree Betti numbers of a Hard Lefschetz manifold are even. 
Proof. Let $\left(M^{2 n}, \omega\right)$ be a symplectic manifold satisfying the Lefschetz property. We us the same idea as in [27, p. 123]. For each $i \in\{0, \ldots, n-1\}$ one has a non-degenerated skew-symmetric bilinear form

$$
\begin{aligned}
& H^{2 i+1}(M, \mathbb{R}) \times H^{2 i+1}(M, \mathbb{R}) \longrightarrow \mathbb{R}, \\
& ([\alpha],[\beta]) \quad \longmapsto\left[\alpha \wedge \beta \wedge \omega^{n-2 i-1}\right]
\end{aligned}
$$

i.e. $H^{2 i+1}(M, \mathbb{R})$ must be even-dimensional.

Obviously, this also proves the next corollary.

Corollary 1.2.4. The first Betti number of a Lefschetz manifold is even.

Finally, the following shows that the statement of the last theorem holds for Kähler manifolds:

Theorem 1.2.5 ([27, p. 122]). Compact Kähler manifolds are Hard Lefschetz.

\section{Nilmanifolds}

We give a brief review of known results about a special kind of solvmanifolds, namely nilmanifolds. For the study non-formal symplectic manifolds, nilmanifolds form one of the best classes. On the one hand, the non-toral nilmanifolds introduce a geometrical complexity, while on the other hand their homotopy theory is still amenable to study. In particular, their minimal models are very easy to calculate and we shall see that each non-toral nilmanifold is non-formal.

A nilmanifold is a compact homogeneous space $G / \Gamma$, where $G$ is a connected and simply-connected nilpotent Lie group and $\Gamma$ a lattice in $G$, i.e. a discrete co-compact subgroup.

Example. Every lattice in the abelian Lie group $\mathbb{R}^{n}$ is isomorphic to $\mathbb{Z}^{n}$. The corresponding nilmanifold is the $n$-dimensional torus.

In contrast to arbitrary solvable Lie groups, there is an easy criterion for nilpotent ones which enables one to decide whether there is a lattice or not.

Recall that the exponential map exp: $\mathfrak{g} \rightarrow G$ of a connected and simplyconnected nilpotent Lie group is a diffeomorphism. We denote its inverse by $\log : G \rightarrow \mathfrak{g}$.

Theorem 2.1 ([50, Theorem 2.12]). A simply-connected nilpotent Lie group $G$ admits a lattice if and only if there exists a basis $\left\{X_{1}, \ldots, X_{n}\right\}$ of the Lie algebra $\mathfrak{g}$ of $G$ such that the structure constants $C_{i j}^{k}$ arising in the brackets

$$
\left[X_{i}, X_{j}\right]=\sum_{k} C_{i j}^{k} X_{k}
$$

are rational numbers.

More precisely we have: 
(i) Let $\mathfrak{g}$ have a basis with respect to which the structure constants are rational. Let $\mathfrak{g}_{\mathbb{Q}}$ be the vector space over $\mathbb{Q}$ spanned by this basis.

Then, if $\mathcal{L}$ is any lattice of maximal rank in $\mathfrak{g}$ contained in $\mathfrak{g}_{\mathbb{Q}}$, the group generated by $\exp (\mathcal{L})$ is a lattice in $G$.

(ii) If $\Gamma$ is a lattice in $G$, then the $\mathbb{Z}$-span of $\log (\Gamma)$ is a lattice $\mathcal{L}$ of maximal rank in the vector space $\mathfrak{g}$ such that the structure constants of $\mathfrak{g}$ with respect to any basis contained in $\mathcal{L}$ belong to $\mathbb{Q}$.

For a given lattice $\Gamma$ in a connected and simply-connected nilpotent Lie group $G$, the subset $\log (\Gamma)$ need not to be an additive subgroup of the Lie algebra $\mathfrak{g}$.

Example. Consider the nilpotent Lie group $G:=\left\{\left(\begin{array}{lll}1 & x & z \\ 0 & 1 & y \\ 0 & 0 & 1\end{array}\right) \mid x, y, z \in \mathbb{R}\right\}$. Its Lie algebra is $\mathfrak{g}:=\left\{\left(\begin{array}{lll}0 & x & z \\ 0 & 0 & y \\ 0 & 0 & 0\end{array}\right) \mid x, y, z \in \mathbb{R}\right\}$, and the logarithm is given by

$$
\log \left(\left(\begin{array}{ccc}
0 & x & z \\
0 & 0 & y \\
0 & 0 & 0
\end{array}\right)\right)=\left(\begin{array}{ccc}
1 & x & z-x y \\
0 & 1 & y \\
0 & 0 & 1
\end{array}\right)
$$

The set of integer matrices contained in $G$ forms a lattice $\Gamma$ in $G$ and

$$
\log (\Gamma)=\left\{\left(\begin{array}{lll}
0 & a & c \\
0 & 0 & b \\
0 & 0 & 0
\end{array}\right) \mid a, b \in \mathbb{Z},(a b \equiv 0(2) \Rightarrow c \in \mathbb{Z}),\left(a b \equiv 1(2) \Rightarrow c \in \frac{1}{2} \mathbb{Z}\right)\right\}
$$

is not a subgroup of $\mathfrak{g}$.

If $\Gamma$ is a lattice such that $\log (\Gamma)$ is a subgroup of the Lie algebra, we call $\Gamma$ a lattice subgroup.

Note that in the context of general Lie groups the name "lattice subgroup" has a different meaning, namely that $G / \Gamma$ has a finite invariant measure. For nilpotent groups and discrete $\Gamma$, the latter is the same as to require that $\Gamma$ is a lattice.

Theorem 2.2 ([12, Theorem 5.4.2]). Let $\Gamma$ be a lattice in a connected and simplyconnected nilpotent Lie group.

(i) $\Gamma$ contains a lattice subgroup of finite index.

(ii) $\Gamma$ is contained as a subgroup of finite index in a lattice subgroup.

For later uses, we quote the following two results. 
Proposition 2.3 ([12, Lemma 5.1.4 (a)]). Let $G$ be a locally compact group, $H$ a closed normal subgroup and $\Gamma$ a discrete subgroup of $G$. Moreover, denote by $\pi: G \rightarrow G / H$ the natural map.

If $\Gamma \cap H$ is a lattice in $H$, and $\Gamma$ is a lattice in $G$, then $\pi(\Gamma)$ is a lattice in $G / H$ and $\Gamma H=H \Gamma$ is a closed subgroup of $G$.

Theorem 2.4 ([12, p. 208]). Let $G$ be a connected and simply-connected nilpotent Lie group with lattice $\Gamma$ and $k \in \mathbb{N}$.

Then $\Gamma \cap D^{(k)} G, \Gamma \cap G^{(k)}$ resp. $\Gamma \cap G_{(k)}$ are lattices in $D^{(k)} G, G^{(k)}$ resp. $G_{(k)}$. Note, $G_{(1)}$ is the center $Z(G)$ of $G$.

We have seen that it is easy to decide if there is a lattice in a given connected and simply-connected nilpotent Lie group, i.e. if it induces a nilmanifold. Moreover, nilmanifolds have very nice properties which will be described now. Below, we shall see that these properties are not satisfied for general solvmanifolds.

Note that we can associate a DGA to each Lie algebra $\mathfrak{g}$ as follows:

Let $\left\{X_{1}, \ldots, X_{n}\right\}$ be a basis of $\mathfrak{g}$ and denote by $\left\{x_{1}, \ldots, x_{n}\right\}$ the dual basis of $\mathfrak{g}^{*}$. The Chevalley-Eilenberg complex of $\mathfrak{g}$ is the differential graded algebra $\left(\bigwedge \mathfrak{g}^{*}, \delta\right)$ with $\delta$ given by

$$
\delta\left(x_{k}\right)=-\sum_{i<j} C_{i j}^{k} x_{i} \wedge x_{j},
$$

where $C_{i j}^{k}$ are the structure constants of $\left\{X_{1}, \ldots, X_{n}\right\}$.

Theorem 2.5 ([46, [48, Theorem 2.1.3]). Let $G / \Gamma$ be a nilmanifold and denote by $\Omega_{l . i .}(G)$ the vector space of left-invariant differential forms on $G$.

Then the natural inclusion $\Omega_{l . i .}(G) \rightarrow \Omega(G / \Gamma)$ induces an isomorphism on cohomology.

Moreover, the minimal model of $G / \Gamma$ is isomorphic to the Chevalley-Eilenberg complex of the Lie algebra of $G$.

Corollary 2.6. Any nilmanifold satisfies $b_{1} \geq 2$.

Proof. Let $\mathfrak{g}$ be a nilpotent Lie algebra. By [61, Theorem 7.4.1] we have $H^{1}\left(\bigwedge \mathfrak{g}^{*}, \delta\right) \cong \mathfrak{g} /[\mathfrak{g}, \mathfrak{g}]$. By [16] any nilpotent Lie algebra $\mathfrak{g}$ satisfies the inequality $\operatorname{dim} \mathfrak{g} /[\mathfrak{g}, \mathfrak{g}] \geq 2$ which then implies $b_{1}(\mathfrak{g}) \geq 2$. Hence the claim follows from the preceding theorem.

We now quote some results that show that it is easy to decide whether a nilmanifold is formal, Kählerian or Hard Lefschetz.

Theorem 2.7 ([29, Theorem 1]). A nilmanifold is formal if and only if it is a torus.

Theorem 2.8 ([48, Theorem 2.2.2]). If a nilmanifold is Kählerian, then it is a torus. 
This theorem follows from Theorem 2.7. Another proof was given by Benson and Gordon in [4]. In fact they proved the following:

Theorem 2.9 ([4, pp. 514 et seq.]). A symplectic non-toral nilmanifold is not Lefschetz.

Corollary 2.10. A symplectic nilmanifold is Hard Lefschetz if and only if it is a torus, independent of the special choice of the symplectic form.

\section{Solvmanifolds in general}

A solvmanifold is a compact homogeneous space $G / \Gamma$, where $G$ is a connected and simply-connected solvable Lie group and $\Gamma$ a lattice in $G$, i.e. a discrete co-compact subgroup.

Remark. It is important to note that there is a more general notion of solvmanifold, namely a compact quotient of a connected and simply-connected solvable Lie group by a (possibly non-discrete) closed Lie subgroup (see [2]), but we are only considering solvmanifolds as in the last definition. Sometimes, such are called special solvmanifolds in the literature.

By [48, Theorem 2.3.11], a solvmanifold in our sense is necessary parallelisable. E.g. the Klein bottle (which can be written as compact homogeneous space of a three-dimensional connected and simply-connected solvable Lie group) is not a solvmanifold covered by our definition.

Obviously, every nilmanifold is also a solvmanifold. But most solvmanifolds are not diffeomorphic to nilmanifolds: Every connected and simply connected solvable Lie group is diffeomorphic to $\mathbb{R}^{m}$ (see e.g. [59]), hence solvmanifolds are aspherical and their fundamental group is isomorphic to the considered lattice. Each lattice in a nilpotent Lie group must be nilpotent. But in general, lattices in solvable Lie group are not nilpotent and therefore the corresponding solvmanifolds are not nilmanifolds.

The fundamental group plays an important role in the study of solvmanifolds.

Theorem 3.1 ([50, Theorem 3.6]). Let $G_{i} / \Gamma_{i}$ be solvmanifolds for $i \in\{1,2\}$ and $\varphi: \Gamma_{1} \rightarrow \Gamma_{2}$ an isomorphism.

Then there exists a diffeomorphism $\Phi: G_{1} \rightarrow G_{2}$ such that

(i) $\left.\Phi\right|_{\Gamma_{1}}=\varphi$,

(ii) $\forall_{\gamma \in \Gamma_{1}} \forall_{p \in G_{1}} \Phi(p \gamma)=\Phi(p) \varphi(\gamma)$.

Corollary 3.2. Two solvmanifolds with isomorphic fundamental groups are diffeomorphic. 
The study of solvmanifolds meets with noticeably greater obstacles than the study of nilmanifolds. Even the construction of solvmanifolds is considerably more difficult than is the case for nilmanifolds. The reason is that there is no simple criterion for the existence of a lattice in a connected and simply-connected solvable Lie group.

We shall quote some necessary criteria.

Proposition 3.3 ([38, Lemma 6.2]). If a connected and simply-connected solvable Lie group admits a lattice then it is unimodular.

Theorem 3.4 ([40], 48, Theorem 3.1.2]). Let $G / \Gamma$ be a solvmanifold that is not a nilmanifold and denote by $N$ the nilradical of $G$.

Then $\Gamma_{N}:=\Gamma \cap N$ is a lattice in $N, \Gamma N=N \Gamma$ is a closed subgroup in $G$ and $G /(N \Gamma)$ is a torus. Therefore, $G / \Gamma$ can be naturally fibred over a non-trivial torus with a nilmanifold as fiber:

$$
N / \Gamma_{N}=(N \Gamma) / \Gamma \longrightarrow G / \Gamma \longrightarrow G /(N \Gamma)=T^{k}
$$

This bundle will be called the Mostow bundle.

\section{Remark.}

(i) The structure group action of the Mostow bundle is given by left translations

$$
N \Gamma / \Gamma_{0} \times N \Gamma / \Gamma \longrightarrow N \Gamma / \Gamma,
$$

where $\Gamma_{0}$ is the largest normal subgroup of $\Gamma$ which is normal in $N \Gamma$. (A proof of the topological version of this fact can be found in [54, Theorem I.8.15]. The proof for the smooth category is analogous.)

(ii) A non-toral nilmanifold $G / \Gamma$ fibers over a non-trivial torus with fibre a nilmanifold, too, since $\Gamma \cap[G, G]$ resp. $\operatorname{im}(\Gamma \rightarrow G /[G, G])$ are lattices in $[G, G] \operatorname{resp} . G /[G, G]$, see above.

In view of Theorem 3.4, we are interested in properties of the nilradical of a solvable Lie group. The following proposition was first proved in [41].

Proposition 3.5. Let $G$ be a solvable Lie group and $N$ its nilradical.

Then $\operatorname{dim} N \geq \frac{1}{2} \operatorname{dim} G$.

Proof. Denote by $\mathfrak{n} \subset \mathfrak{g}$ the Lie algebras of $N \subset G$ and by $\mathfrak{n}_{\mathbb{C}} \subset \mathfrak{g}_{\mathbb{C}}$ their complexifications. Note that $\mathfrak{g}_{\mathbb{C}}$ is solvable with nilradical $\mathfrak{n}_{\mathbb{C}}$, so from [59, Corollary 3.8.4] it follows that $\mathfrak{n}_{\mathbb{C}}=\left\{X \in \mathfrak{g}_{\mathbb{C}}\left|a_{\mid}\right|_{\left[\mathfrak{g}_{\mathbb{C}}, \mathfrak{g}_{\mathbb{C}}\right]}\right.$ nilpotent $\}$. Therefore, since $\operatorname{ad}: \mathfrak{g}_{\mathbb{C}} \rightarrow \operatorname{Aut}\left(\left[\mathfrak{g}_{\mathbb{C}}, \mathfrak{g}_{\mathbb{C}}\right]\right)$ is a representation of $\mathfrak{g}_{\mathbb{C}}$ in $\left[\mathfrak{g}_{\mathbb{C}}, \mathfrak{g}_{\mathbb{C}}\right]$, by Lie's theorem (e.g. [59, Theorem 3.7.3]) there exist $\lambda_{1}, \ldots, \lambda_{k} \in \mathfrak{g}_{\mathbb{C}}^{*}$ such that $\mathfrak{n}_{\mathbb{C}}=\bigcap_{i=1}^{k} \operatorname{ker} \lambda_{i}$, where $k:=\operatorname{dim}_{\mathbb{C}}\left[\mathfrak{g}_{\mathbb{C}}, \mathfrak{g}_{\mathbb{C}}\right]$. 
A straightforward calculation shows $\operatorname{dim}_{\mathbb{C}} \bigcap_{i=1}^{k} \operatorname{ker} \lambda_{i} \geq \operatorname{dim}_{\mathbb{C}} \mathfrak{g}_{\mathbb{C}}-k$, so we have proven: $\operatorname{dim}_{\mathbb{C}} \mathfrak{n}_{\mathbb{C}} \geq \operatorname{dim}_{\mathbb{C}} \mathfrak{g}_{\mathbb{C}}-\operatorname{dim}_{\mathbb{C}}\left[\mathfrak{g}_{\mathbb{C}}, \mathfrak{g}_{\mathbb{C}}\right]$.

Because $\mathfrak{g}_{\mathbb{C}}$ is solvable, we get by [59, Corollary 3.8.4] that $\left[\mathfrak{g}_{\mathbb{C}}, \mathfrak{g}_{\mathbb{C}}\right] \subset \mathfrak{n}_{\mathbb{C}}$ and hence $\operatorname{dim}_{\mathbb{C}} \mathfrak{n}_{\mathbb{C}} \geq \operatorname{dim}_{\mathbb{C}} \mathfrak{g}_{\mathbb{C}}-\operatorname{dim}_{\mathbb{C}} \mathfrak{n}_{\mathbb{C}}$, i.e.

$$
2 \operatorname{dim}_{\mathbb{C}} \mathfrak{n}_{\mathbb{C}} \geq \operatorname{dim}_{\mathbb{C}} \mathfrak{g}_{\mathbb{C}}
$$

The proposition now follows from $\operatorname{dim}_{\mathbb{R}} \mathfrak{g}=\operatorname{dim}_{\mathbb{C}} \mathfrak{g}_{\mathbb{C}}$ and $\operatorname{dim}_{\mathbb{R}} \mathfrak{n}=\operatorname{dim}_{\mathbb{C}} \mathfrak{n}_{\mathbb{C}}$

In some cases, we will be able to apply the next theorem to the situation of Theorem 3.4. It then gives a sufficient condition for the Mostow bundle to be a principal bundle.

Theorem 3.6. Let $G$ be a connected and simply-connected solvable Lie group and $\Gamma$ a lattice in $G$. Suppose that $\{e\} \neq H \varsubsetneqq G$ is a closed normal abelian Lie subgroup of $G$ with $H \subset N(\Gamma)$, the normalizer of $\Gamma$. (For example the latter is satisfied if $H$ is central.) Assume further that $\Gamma_{H}:=\Gamma \cap H$ is a lattice in $H$.

Then $H / \Gamma_{H}=H \Gamma / \Gamma$ is a torus and

$$
H / \Gamma_{H} \longrightarrow G / \Gamma \longrightarrow G / H \Gamma
$$

is a principal torus bundle over a solvmanifold.

Proof. By assumption, $H$ is a closed normal abelian subgroup of $G$ and $\Gamma_{H}$ is a lattice in $H$. We have for $h_{1} \gamma_{1}, h_{2} \gamma_{2} \in H \Gamma$ with $h_{i} \in H, \gamma_{i} \in \Gamma$ the equivalence

$$
\left(h_{1} \gamma_{1}\right)^{-1}\left(h_{2} \gamma_{2}\right) \in \Gamma \Leftrightarrow h_{1}^{-1} h_{2} \in \Gamma_{H},
$$

i.e. $H / \Gamma_{H}=H \Gamma / \Gamma$. Therefore, Proposition 2.3 implies that (1) is a fibre bundle whose fibre is clearly a torus and its base a solvmanifold. The structure group action is given by the left translations

$$
H \Gamma / \Gamma_{0} \times H \Gamma / \Gamma \longrightarrow H \Gamma / \Gamma,
$$

where $\Gamma_{0}$ is the largest normal subgroup of $\Gamma$ which is normal in $H \Gamma$. (This can be seen analogous as in Remark (i) on page 12.) Since $H$ is contained in $N(\Gamma)=\left\{g \in G \mid g \Gamma g^{-1}=\Gamma\right\}$, we have for each $h \in H$ and $\gamma, \gamma_{0} \in \Gamma$

$$
(h \gamma) \gamma_{0}(h \gamma)^{-1}=h \gamma \gamma_{0} \gamma^{-1} h^{-1} \in h \Gamma h^{-1}=\Gamma,
$$

i.e. $\Gamma=\Gamma_{0}$ and the theorem follows.

We have seen that the Chevalley-Eilenberg complex associated to a nilmanifold is its minimal model. In this respect, arbitrary solvmanifolds differ in an essential way from nilmanifolds. However, in the special case of a solvmanifold which is the quotient of completely solvable Lie group, one has an access to the minimal model. 
Definition 3.7. Let $G$ be a Lie group with Lie algebra $\mathfrak{g}$.

(i) $G$ and $\mathfrak{g}$ are called completely solvable if the linear map ad $X: \mathfrak{g} \rightarrow \mathfrak{g}$ has only real root 1 for all $X \in \mathfrak{g}$.

(ii) If $G$ is simply-connected and exp: $\mathfrak{g} \rightarrow G$ is a diffeomorphism, then $G$ is called exponential .

Remark. In the literature a connected and simply-connected Lie group is sometimes called exponential if the exponential map is surjective. This is weaker than our definition.

A nilpotent Lie group or algebra is completely solvable, and it is easy to see that completely solvable Lie groups or algebras are solvable. Moreover, the two propositions below show that simply-connected completely solvable Lie groups are exponential, and exponential Lie groups are solvable. Note that the second proposition is simply a reformulation of results of Saitô and Dixmier.

Proposition 3.8 ([49, Theorem 2.6.3]). Any exponential Lie group is solvable.

Proposition 3.9. A connected and simply-connected solvable Lie group $G$ with Lie algebra $\mathfrak{g}$ is exponential if and only if the linear map ad $X: \mathfrak{g} \rightarrow \mathfrak{g}$ has no purely imaginary roots for all $X \in \mathfrak{g}$.

Proof. Let $G$ be a solvable Lie group. By [51, Théorème II.1], ad $X$ has no purely imaginary roots for all $X \in \mathfrak{g}$ if and only if the exponential map is surjective. If this is the case, [51, Théorème I.1] implies that the exponential map is even bijective. For solvable Lie groups, the statement " $\left(1^{\circ}\right) \Leftrightarrow\left(2^{\circ}\right)$ " of [17. Théorème 3] says that this is equivalent to the exponential map being a diffeomorphism.

Let a lattice in a connected and simply-connected solvable Lie group be given. Then Theorem 3.4 stated that its intersection with the nilradical is a lattice in the nilradical. In the case of completely solvable Lie groups, we have an analogue for the commutator.

Proposition 3.10 ([26, Proposition 1]). Let $G$ be a connected and simply-connected completely solvable Lie group and $\Gamma$ a lattice in $G$.

Then $[\Gamma, \Gamma]$ is a lattice in $[G, G]$. In particular, $\Gamma \cap[G, G]$ is a lattice in $[G, G]$.

We formulate the result that enables us to compute the minimal model of solvmanifolds which are built by dividing a lattice out of a completely solvable group. The main part of the next theorem is due to Hattori [33].

\footnotetext{
${ }^{1} \mathrm{By}$ a root of a linear map, we mean a (possibly non-real) root of the characteristic polynomial.
} 
Theorem 3.11. Let $G / \Gamma$ be a solvmanifold. Denote by $\left(\bigwedge \mathfrak{g}^{*}, \delta\right)$ the ChevalleyEilenberg complex of $G$ and recall that $\mathfrak{g}^{*}$ is the set of left-invariant differential 1 -forms on $G$. Then the following holds:

(i) The natural inclusion $\left(\bigwedge \mathfrak{g}^{*}, \delta\right) \rightarrow(\Omega(G / \Gamma), d)$ induces an injection on cohomology.

(ii) If $G$ is completely solvable, then the inclusion in (i) is a quasi-isomorphism, i.e. it induces an isomorphism on cohomology. Therefore, the minimal model $\mathcal{M}_{G / \Gamma}$ is isomorphic to the minimal model of the Chevalley-Eilenberg complex.

(iii) If $\operatorname{Ad}(\Gamma)$ and $\operatorname{Ad}(G)$ have the same Zariski closure.2, then the inclusion in (i) is a quasi-isomorphism.

Proof. (i) is [48, Theorem 3.2.10] and (iii) is [50, Corollary 7.29].

ad (ii): Denote the mentioned inclusion by $i:\left(\bigwedge \mathfrak{g}^{*}, \delta\right) \rightarrow(\Omega(G / \Gamma), d)$. By Hattori's Theorem (see [48, p. 77]), this is a quasi-isomorphism. It remains to show that the minimal model $\rho:\left(\mathcal{M}_{C E}, \delta_{C E}\right) \rightarrow\left(\bigwedge \mathfrak{g}^{*}, \delta\right)$ of $\left(\bigwedge \mathfrak{g}^{*}, \delta\right)$ is the minimal model of $(\Omega(G / \Gamma), d)$. Since the minimal model is unique and the map $i \circ \rho:\left(\mathcal{M}_{C E}, \delta_{C E}\right) \rightarrow(\Omega(G / \Gamma), d)$ is a quasi-isomorphism, this is obvious.

There are examples where the inclusion in (i) in the last theorem is not a quasi-isomorphism: Consider the Lie group $G$ which is $\mathbb{R}^{3}$ as a manifold and whose Lie group structure is given by

$(s, a, b) \cdot(t, x, y)=(s+t, \cos (2 \pi t) a-\sin (2 \pi t) b+x, \sin (2 \pi t) a+\cos (2 \pi t) b+y)$.

$G$ is not completely solvable and one calculates for its Lie algebra $b_{1}(\mathfrak{g})=1$. $G$ contains the abelian lattice $\Gamma:=\mathbb{Z}^{3}$ and $G / \Gamma$ is the 3 -torus which has $b_{1}=3$.

We have seen in the last section that the first Betti number of a nilmanifold is greater than or equal to two. For arbitrary solvmanifolds this is no longer true. Below, we shall see various examples of solvmanifolds with $b_{1}=1$. The following corollary shows that $b_{1}=0$ cannot arise.

Corollary 3.12. Any solvmanifold satisfies $b_{1} \geq 1$.

Proof. Let $\mathfrak{g}$ be a solvable Lie algebra. As in the nilpotent case we have $b_{1}\left(\bigwedge \mathfrak{g}^{*}, \delta\right)=\operatorname{dim} \mathfrak{g} /[\mathfrak{g}, \mathfrak{g}]$, and $\operatorname{dim} \mathfrak{g} /[\mathfrak{g}, \mathfrak{g}] \geq 1$ by solvability. The claim now follows from Theorem 3.11 (i).

\footnotetext{
${ }^{2} \mathrm{~A}$ basis for the Zariski topology on $\mathrm{GL}(m, \mathbb{C})$ is given by the sets

$$
U_{p}:=\mathrm{GL}(m, \mathbb{C}) \backslash p^{-1}(\{0\}),
$$
}

where $p$ : $\mathrm{GL}(m, \mathbb{C}) \cong \mathbb{C}^{\left(m^{2}\right)} \rightarrow \mathbb{C}$ ranges over polynomials. 
To end this section, we shortly discuss the existence problem for Kähler structures on solvmanifolds. The only Kählerian nilmanifolds are tori, but in the general context we have the hyperelliptic surfaces, which are non-toral Kählerian solvmanifolds, see Section 6 below. ([48, Theorem 3.4.1] states the only Kählerian solvmanifolds in dimension four are tori. This is not correct, as first noted by Hasegawa in [32].) Benson and Gordon [5] conjectured in 1990 that the existence of a Kähler structure on a solvmanifold $G / \Gamma$ with $G$ completely solvable forces $G / \Gamma$ to be toral and this is true. In fact, Hasegawa proved in the first half of this decade the following:

Theorem 3.13 ([32]). A solvmanifold $G / \Gamma$ is Kählerian if and only if it is a finite quotient of a complex torus which has a structure of a complex torus bundle over a complex torus.

If $G$ is completely solvable, then $G / \Gamma$ is Kählerian if and only if it is a complex torus.

In later sections we shall see that neither the Hard Lefschetz property nor formality is sufficient for an even-dimensional solvmanifold to be Kählerian.

\section{Semidirect products}

In later sections we shall try to examine low-dimensional solvmanifolds. Concerning this, a first step is to use the known classification of the (connected and simply-connected) low-dimensional solvable Lie groups. Most of them have the structure of semidirect products. In order to define this notion, we recall the construction of the Lie group structure of the group of Lie group automorphisms of a simply-connected Lie group in the following theorem. It collects results that can be found in [60, pp. 117 et seq.].

\section{Theorem 4.1.}

(i) Let $\mathfrak{h}=\left(|\mathfrak{h}|=\mathbb{R}^{h},[\ldots, \ldots]\right)$ be an h-dimensional Lie algebra. Then the set $\mathrm{A}(\mathfrak{h})$ of Lie algebra isomorphisms of $\mathfrak{h}$ is a closed Lie subgroup of the automorphism group Aut $(|\mathfrak{h}|)$ of the $h$-dimensional vector space $|\mathfrak{h}|$. The Lie algebra of $\mathrm{A}(\mathfrak{h})$ is

$$
\mathfrak{d}(\mathfrak{h})=\{\varphi \in \operatorname{End}(|\mathfrak{h}|) \mid \varphi \text { derivation with respect to }[\ldots, \ldots]\} .
$$

(ii) Let $H$ be a connected and simply-connected Lie group with neutral element $e$ and Lie algebra $\mathfrak{h}$. The Lie group structure of $\mathrm{A}(H)$, the group of Lie group automorphisms of $H$, is given by the following group isomorphism:

$$
\mathrm{A}(H) \longrightarrow \mathrm{A}(\mathfrak{h}), f \longmapsto d_{e} f
$$


Moreover, if $H$ is exponential, its inverse is the map

$$
\mathrm{A}(\mathfrak{h}) \longrightarrow \mathrm{A}(H), \varphi \longmapsto \exp ^{H} \circ \varphi \circ \log ^{H} .
$$

For given (Lie) groups $G, H$ and a (smooth) action $\mu: G \times H \rightarrow H$ by (Lie) group automorphisms, one defines the semidirect product of $G$ and $H$ via $\mu$ as the (Lie) group $G \ltimes_{\mu} H$ with underlying set (manifold) $G \times H$ and group structure defined as follows:

$$
\forall_{\left(g_{1}, h_{1}\right),\left(g_{2}, h_{2}\right) \in G \times H}\left(g_{1}, h_{1}\right)\left(g_{2}, h_{2}\right)=\left(g_{1} g_{2}, \mu\left(g_{2}^{-1}, h_{1}\right) h_{2}\right)
$$

Note that for $(g, h) \in G \ltimes_{\mu} H$ we have $(g, h)^{-1}=\left(g^{-1}, \mu\left(g, h^{-1}\right)\right)$.

If the action $\mu$ is trivial, i.e. $\forall_{g \in G, h \in H} \mu(g, h)=h$, one obtains the ordinary direct product. In the case of Lie groups $G$ and $H$, the exponential map $\exp ^{G \times H}$ is known to be the direct product of $\exp ^{G}$ and $\exp ^{H}$. If the action is not trivial, the situation becomes a little more complicated:

Theorem 4.2. Let $G, H$ be connected Lie groups and $\mu: G \times H \rightarrow H$ a smooth action by Lie group automorphisms. Denote the Lie algebras of $G$ and $H$ by $\mathfrak{g}$ and $\mathfrak{h}$ and let $\phi:=\left(d_{e_{G}} \mu_{1}\right): \mathfrak{g} \rightarrow \mathfrak{d}(\mathfrak{h})$, where $\mu_{1}: G \rightarrow \mathrm{A}(\mathfrak{h})$ is given by $\mu_{1}(g)=d_{e_{H}} \mu(g, \ldots)=\operatorname{Ad}_{g}^{G \ltimes \mu H}$.

(i) The Lie algebra of $G \ltimes_{\mu} H$ is $\mathfrak{g} \ltimes_{\phi} \mathfrak{h}$. This Lie algebra is called semidirect product of $\mathfrak{g}$ and $\mathfrak{h}$ via $\phi$. Its underlying vector space is $\mathfrak{g} \times \mathfrak{h}$ and the bracket for $\left(X_{1}, Y_{1}\right),\left(X_{2}, Y_{2}\right) \in \mathfrak{g} \times \mathfrak{h}$ is given by

$$
\left[\left(X_{1}, Y_{1}\right),\left(X_{2}, Y_{2}\right)\right]=\left(\left[X_{1}, X_{2}\right]_{\mathfrak{g}},\left[Y_{1}, Y_{2}\right]_{\mathfrak{h}}+\phi\left(X_{1}\right)\left(Y_{2}\right)-\phi\left(X_{2}\right)\left(Y_{1}\right)\right) .
$$

In the sequel we shall identify $X \equiv(X, 0)$ and $Y \equiv(0, Y)$.

(ii) For $(X, Y) \in \mathfrak{g} \ltimes_{\phi} \mathfrak{h}$ one has $\exp ^{G \ltimes_{\mu} H}((X, Y))=\left(\exp ^{G}(X), \gamma(1)\right)$, where $\gamma: \mathbb{R} \rightarrow H$ is the solution of

$$
\dot{\gamma}(t)=\left(d_{e_{H}} R_{\gamma(t)}\right)\left(\exp ^{A(\mathfrak{h})}\left(-\left.t \operatorname{ad}(X)\right|_{\mathfrak{h}}\right)(Y)\right), \quad \gamma(0)=e_{H} .
$$

Here $R_{a}$ denotes the right translation by an element $a \in H$.

Proof. The proof of (i) can be found in [59]. We give a proof of (ii). Given a Lie group homomorphism $f$ between Lie groups, we denote its differential at the neutral element by $f_{*}$.

For $\left(g_{0}, h_{0}\right),(g, h) \in G \ltimes_{\mu} H$ we have $R_{\left(g_{0}, h_{0}\right)}(g, h)=\left(R_{g_{0}}(g), R_{h_{0}}\left(\mu\left(g_{0}^{-1}, h\right)\right)\right.$, and this yields for $(X, Y) \in \mathfrak{g} \ltimes_{\phi} \mathfrak{h}$

$$
\left(R_{\left(g_{0}, h_{0}\right)}\right)_{*}((X, Y))=\left(\left(R_{g_{0}}\right)_{*}(X),\left(R_{h_{0}}\right)_{*}\left(\mu_{1}\left(g_{0}^{-1}\right)(Y)\right)\right) .
$$


Since $\left(\gamma_{1}(t), \gamma_{2}(t)\right):=\exp ^{G \ltimes_{\mu} H}(t(X, Y))$ is the integral curve through the identity of both the right- and left-invariant vector fields associated to $(X, Y)$, the last equation implies that $\left(\gamma_{1}(t), \gamma_{2}(t)\right)$ is the solution of the following differential equations:

$$
\begin{array}{rlrl}
\gamma_{1}(0) & =e_{G}, & \dot{\gamma}_{1}(t)=\left(R_{\gamma_{1}(t)}\right)_{*}(X), \\
\gamma_{2}(0)=e_{H}, & \dot{\gamma}_{2}(t)=\left(R_{\gamma_{2}(t)}\right)_{*}\left(\mu_{1}\left(\gamma_{1}(-t)\right)(Y)\right) .
\end{array}
$$

$\gamma_{1}(t)=\exp ^{G}(t X)$ is the solution of (2), and this implies

$$
\mu_{1}\left(\gamma_{1}(-t)\right)=\left.\operatorname{Ad}_{\gamma_{1}(-t)}^{G \ltimes_{\mu} H}\right|_{\mathfrak{h}}=\exp ^{A(\mathfrak{h})}\left(-\left.t \operatorname{ad}(X)\right|_{\mathfrak{h}}\right),
$$

i.e. (3) is equivalent to $\gamma_{2}(0)=e_{H}, \dot{\gamma}_{2}(t)=\left(R_{\gamma_{2}(t)}\right)_{*}\left(\exp ^{A(\mathfrak{h})}\left(-\left.t \operatorname{ad}(X)\right|_{\mathfrak{h}}\right)(Y)\right)$. So the theorem is proven.

A connected and simply-connected solvable Lie group $G$ with nilradical $N$ is called almost nilpotent if it can be written as $G=\mathbb{R} \ltimes_{\mu} N$. Moreover, if $N$ is abelian, i.e. $N=\mathbb{R}^{n}$, then $G$ is called almost abelian.

Let $G=\mathbb{R} \ltimes_{\mu} N$ be an almost nilpotent Lie group. Since $N$ has codimension one in $G$, we can consider $\mu$ as a one-parameter group $\mathbb{R} \rightarrow \mathrm{A}(N)$. By Theorem 4.1, there exists $\varphi \in \mathfrak{d}(\mathfrak{n})$ with

$$
\forall t \in \mathbb{R} \mu(t)=\exp ^{N} \circ \exp ^{A u t(|\mathfrak{n}|)}(t \varphi) \circ \log ^{N} .
$$

Choosing a basis of $|\mathfrak{n}|$, we can identify $\operatorname{Aut}(|\mathfrak{n}|)$ with a subset of $\mathfrak{g l}(n, \mathbb{R})$ and get

$$
\forall_{t \in \mathbb{R}} d_{e}(\mu(t)) \in \exp ^{G L(n, \mathbb{R})}(\mathfrak{g l}(n, \mathbb{R})) .
$$

Note, if $N$ is abelian, the exponential map $\exp ^{N}: \mathfrak{n} \rightarrow N$ is the identity. These considerations make it interesting to examine the image of $\exp ^{G L(n, \mathbb{R})}$.

Theorem $4.3\left(\left[47\right.\right.$, Theorem 6]). $M$ is an element of $\exp ^{G L(n, \mathbb{R})}(\mathfrak{g l}(n, \mathbb{R}))$ if and only if the real Jordan form of $M$ contains in the form of pairs the blocks belonging to real negative eigenvalues $\lambda_{i}^{-}$, whenever there exist real negative eigenvalues $\lambda_{i}^{-}$ of $M$. I.e. the block belonging to such $a \lambda_{i}^{-}$is of the following form

$$
\bigoplus_{j=1}^{n_{i}}\left(\begin{array}{cc}
J_{n_{i j}} & 0 \\
0 & J_{n_{i j}}
\end{array}\right)
$$

with

$$
J_{n_{i j}}=\left(\begin{array}{cccc}
\lambda_{i}^{-} & 1 & & 0 \\
& \lambda_{i}^{-} & \ddots & \\
& & \ddots & 1 \\
0 & & & \lambda_{i}^{-}
\end{array}\right) \in \mathrm{M}\left(n_{i j}, n_{i j} ; \mathbb{R}\right)
$$


We are now going to derive some facts that follow from the existence of a lattice in an almost nilpotent Lie group.

Theorem 4.4 ([57]). Let $G=\mathbb{R} \ltimes_{\mu} N$ be an almost nilpotent and completely solvable Lie group containing a lattice $\Gamma$.

Then there is a one-parameter group $\nu: \mathbb{R} \rightarrow \mathrm{A}(N)$ such that $\nu(k)$ preserves the lattice $\Gamma_{N}:=\Gamma \cap N$ for all $k \in \mathbb{Z} . \Gamma$ is isomorphic to $\mathbb{Z} \ltimes_{\nu} \Gamma_{N}$ and $G / \Gamma$ is diffeomorphic to $\left(\mathbb{R} \ltimes{ }_{\nu} N\right) /\left(\mathbb{Z} \ltimes_{\nu} \Gamma_{N}\right)$.

Moreover, there are $t_{1} \in \mathbb{R} \backslash\{0\}$ and an inner automorphism $I_{n_{1}} \in \mathrm{A}(N)$ such that $\nu(1)=\mu\left(t_{1}\right) \circ I_{n_{1}}$.

Proof. We know that $\Gamma_{N}$ is a lattice in $N$ and $\operatorname{im}(\Gamma \rightarrow G / N) \cong \Gamma / \Gamma_{N}$ is a lattice in $G / N \cong \mathbb{R}$. Therefore, $\Gamma / \Gamma_{N} \cong \mathbb{Z}$ is free, and the following exact sequence is split:

$$
\{1\} \longrightarrow \Gamma_{N} \longrightarrow \Gamma \longrightarrow \mathbb{Z} \longrightarrow\{0\}
$$

i.e. there is a group-theoretic section $s: \mathbb{Z} \rightarrow \Gamma$. [51, Théorème II.5] states that a group homomorphism from a lattice of completely solvable Lie group into another completely solvable Lie group uniquely extends to a Lie group homomorphism of the Lie groups. Hence, $s$ extends uniquely to a one-parameter group $s: \mathbb{R} \rightarrow G$. Therefore,

$$
\nu: \mathbb{R} \longrightarrow \mathrm{A}(N), \quad \nu(t)(n)=s(t) \cdot n \cdot s(t)^{-1}
$$

is a one-parameter group with $\forall_{k \in \mathbb{Z}} \nu(k)\left(\Gamma_{N}\right)=\Gamma_{N}$, the lattice $\Gamma$ is isomorphic to $\mathbb{Z} \ltimes_{\nu} \Gamma_{N}$ and $G / \Gamma$ is diffeomorphic to $\left(\mathbb{R} \ltimes_{\nu} N\right) /\left(\mathbb{Z} \ltimes_{\nu} \Gamma_{N}\right)$.

Let $\gamma_{1}:=s(1) \in\left(\Gamma \backslash \Gamma_{N}\right) \subset \mathbb{R} \ltimes_{\mu} N$. There are unique $t_{1} \in \mathbb{R} \backslash\{0\}, n_{1} \in N$ with $\gamma_{1}=t_{1} \cdot n_{1}$, where we identify $t_{1} \equiv\left(t_{1}, e_{N}\right) \in G$ and $n_{1} \equiv\left(0, n_{1}\right) \in G$. Since $G=\mathbb{R} \ltimes_{\nu} N$ and $G=\mathbb{R} \ltimes_{\mu} N$ with the same normal subgroup $N$ of $G$, one has for all $n \in N$

$$
\nu(1)(n)=\gamma_{1} \cdot n \cdot \gamma_{1}^{-1}=t_{1} \cdot n_{1} \cdot n \cdot n_{1}^{-1} \cdot t_{1}^{-1}=\mu\left(t_{1}\right)\left(n_{1} \cdot n \cdot n_{1}^{-1}\right)=\mu\left(t_{1}\right)\left(I_{n_{1}}(n)\right),
$$

from where the theorem follows.

Corollary 4.5. Let $G=\mathbb{R} \ltimes_{\mu} N$ be an almost nilpotent (not necessary completely solvable) Lie group containing a lattice $\Gamma$. Again, denote by $\Gamma_{N}:=\Gamma \cap N$ the induced lattice in the nilradical of $G$.

Then there exist $t_{1} \in \mathbb{R} \backslash\{0\}$, a group homomorphism $\nu: \mathbb{Z} \rightarrow \operatorname{Aut}\left(\Gamma_{N}\right)$, and an inner automorphism $I_{n_{1}}$ of $N$ such that $\Gamma \cong \mathbb{Z} \ltimes_{\nu} \Gamma_{N}$ and $\nu(1)=\mu\left(t_{1}\right) \circ I_{n_{1}}$.

If $G$ is almost abelian, then a basis transformation yields $\Gamma \cong t_{1} \mathbb{Z} \ltimes_{\left.\mu\right|_{\mathbb{Z}^{n}}} \mathbb{Z}^{n}$.

Proof. We argue as in the last proof. But we do not use [51, Théorème 5] and get only a group homomorphism $\nu: \mathbb{Z} \rightarrow \operatorname{Aut}\left(\Gamma_{N}\right)$ (defined on $\mathbb{Z}$ instead of $\mathbb{R}$ ). For general $N$, the calculation at the end of the proof implies the claim.

Since an abelian group has only one inner automorphism, in the almost abelian case this yields $\nu(1)=\left.\mu\left(t_{1}\right)\right|_{\Gamma_{N}}$, so $\nu$ can be extended to $\nu: \mathbb{R} \rightarrow \mathrm{A}\left(\mathbb{R}^{n}\right)$ via 
$\nu(t):=\mu\left(t \cdot t_{1}\right)$. Further, by Corollary 3.2, we have $\Gamma_{N} \cong \mathbb{Z}^{n}$.

Hence we have seen, that the existence of a lattice in an almost nilpotent Lie group implies that a certain Lie group automorphism must preserve a lattice in the (nilpotent) nilradical. The next theorem deals with such automorphisms.

Theorem 4.6. Let $N$ be a connected and simply-connected nilpotent Lie group with Lie algebra $\mathfrak{n}, f_{*} \in \mathrm{A}(\mathfrak{n})$, and $f:=\exp ^{N} \circ f_{*} \circ \log ^{N} \in \mathrm{A}(N)$, i.e. $d_{e} f=f_{*}$. Assume that $f$ preserves a lattice $\Gamma$ in $N$.

Then there exists a basis $\mathfrak{X}$ of $\mathfrak{n}$ such that $M_{\mathfrak{X}}\left(f_{*}\right) \in \mathrm{GL}(n, \mathbb{Z})$, where $M_{\mathfrak{X}}\left(f_{*}\right)$ denotes the matrix of $f_{*}$ with respect to $\mathfrak{X}$.

Moreover, if there are a one-parameter group $\mu: \mathbb{R} \rightarrow \mathrm{A}(N)$ and $t_{0} \neq 0$ such that $\mu\left(t_{0}\right)=f$, i.e. $d_{e}\left(\mu\left(t_{0}\right)\right)=f_{*}$, then $\operatorname{det}\left(d_{e}(\mu(\ldots))\right) \equiv 1$.

Proof. By Theorem 2.1 (ii),

$$
\mathcal{L}:=\left\langle\log ^{N}(\Gamma)\right\rangle_{\mathbb{Z}}=\left\{\sum_{i=1}^{m} k_{i} V_{i} \mid m \in \mathbb{N}_{+}, k_{i} \in \mathbb{Z}, V_{i} \in \log ^{N}(\Gamma)\right\}
$$

is a lattice in $\mathfrak{n}$. Therefore, there exists a basis $\mathfrak{X}=\left\{X_{1}, \ldots, X_{n}\right\}$ of $\mathfrak{n}$ such that $\mathcal{L}=\langle\mathfrak{X}\rangle_{\mathbb{Z}}$.

Since $f(\Gamma) \subset \Gamma$, we have $f_{*}\left(\log ^{N}(\Gamma)\right) \subset \log ^{N}(\Gamma)$. This implies $f_{*}(\mathcal{L}) \subset \mathcal{L}$ and hence, $M_{\mathfrak{X}}\left(f_{*}\right) \in \mathrm{GL}(n, \mathbb{Z})$.

Further, if $\mu\left(t_{0}\right)=f$ with $\mu, t_{0} \neq 0$ as in the statement of the theorem, then the map $\Delta:=\operatorname{det}_{\operatorname{cod}}(\mu(\ldots)):(\mathbb{R},+) \rightarrow(\mathbb{R} \backslash\{0\}, \cdot)$ is a continuous group homomorphism with $\Delta(0)=1$ and $\Delta\left(t_{0}\right)= \pm 1$, i.e. $\Delta \equiv 1$.

Remark. The basis $\mathfrak{X}$ in the last theorem has rational structure constants.

Obviously, a one-parameter group $\mu$ in the automorphism group of an abelian Lie group with $\mu\left(t_{0}\right)$ integer valued for $t_{0} \neq 0$ defines a lattice in $\mathbb{R} \ltimes_{\mu} \mathbb{R}^{n}$. It is easy to compute the first Betti number of the corresponding solvmanifold, as the next proposition will show. Before stating it, we mention that the situation becomes more complicated in the case of a non-abelian and nilpotent group $N$.

Let a one-parameter group $\mu: \mathbb{R} \rightarrow \mathrm{A}(N)$ be given and $t_{0} \neq 0$ such that $d_{e}\left(\mu\left(t_{0}\right)\right)$ is an integer matrix with respect to a basis $\mathfrak{X}$ of the Lie algebra $\mathfrak{n}$ of $N$. In general, this does not enable us to define a lattice in $\mathbb{R} \ltimes_{\mu} N$. But if $\Gamma_{N}:=\exp ^{N}\left(\langle\mathfrak{X}\rangle_{\mathbb{Z}}\right)$ is a lattice in $N$, i.e. $\Gamma_{N}$ is a lattice group, then this is possible.

Proposition 4.7. Let $\mu: \mathbb{R} \rightarrow \mathrm{SL}(n, \mathbb{R})$ be a one-parameter group such that $\mu(1)=\left(m_{i j}\right)_{i, j} \in \mathrm{SL}(n, \mathbb{Z})$.

Then $M:=\left(\mathbb{R} \ltimes_{\mu} \mathbb{R}^{n}\right) /\left(\mathbb{Z} \ltimes_{\mu} \mathbb{Z}^{n}\right)$ is a solvmanifold with

$$
\pi_{1}(M)=\left\langle e_{0}, e_{1}, \ldots, e_{n} \mid \begin{array}{c}
\forall i \in\{1, \ldots, n\} \\
\left.\forall_{i, j \in\{1, \ldots, n\}} e_{0} e_{i} e_{0}^{-1}=e_{i}^{m_{1 i}}, e_{j}\right]=1
\end{array}\right\rangle
$$

and $b_{1}(M)=n+1-\operatorname{rank}(\mu(1)-\mathrm{id})$. 
Proof. The statement about the fundamental group is clear. Therefore, we get

$$
\begin{aligned}
& H_{1}(M, \mathbb{Z})=\left\langle e_{0}, e_{1}, \ldots, e_{n}\right| \quad \forall_{i \in\{1, \ldots, n\}} e_{1}^{m_{1 i}} \cdots e_{i}^{m_{i i}-1} \cdots e_{n}^{m_{n i}}=1 \\
& \left.\forall_{i, j \in\{0, \ldots, n\}}\left[e_{i}, e_{j}\right]=1 \quad\right\rangle
\end{aligned}
$$

and this group is the abelianisation of

$$
\mathbb{Z} \oplus\left\langle e_{1}, \ldots, e_{n} \mid \forall_{i \in\{1, \ldots, n\}} e_{1}^{m_{1 i}} \cdots e_{i}^{m_{i i}-1} \cdots e_{n}^{m_{n i}}=1\right\rangle .
$$

Now, the proof of the theorem about finitely generated abelian groups (see e.g. [7]) shows $H_{1}(M, \mathbb{Z})=\mathbb{Z}^{n-k+1} \oplus \bigoplus_{i=1}^{k} \mathbb{Z}_{d_{i}}$, where $d_{1}, \ldots, d_{k} \in \mathbb{N}_{+}$denote the elementary divisors of $\mu(1)-\mathrm{id}$. The proposition follows.

We finally mention a result of Gorbatsevich. In view of Theorem 3.11 (iii), it enables us to compute the minimal model of a wide class of solvmanifolds which are discrete quotients of almost abelian Lie groups.

Theorem $4.8([26$, Theorem 4]). Let $\mu: \mathbb{R} \rightarrow \operatorname{SL}(n, \mathbb{R})$ be a one-parameter group such that $\mu(1)=\exp ^{S L(n, \mathbb{R})}(\dot{\mu}(0)) \in \mathrm{SL}(n, \mathbb{Z})$. Denote by $\lambda_{1}, \ldots, \lambda_{n}$ the (possibly not pairwise different) roots of $\dot{\mu}(0)$. Then $\Gamma:=\left(\mathbb{Z} \ltimes_{\mu} \mathbb{Z}^{n}\right)$ is a lattice in $G:=\left(\mathbb{R} \ltimes_{\mu} \mathbb{R}^{n}\right)$.

The Zariski closures of $\operatorname{Ad}(\Gamma)$ and $\operatorname{Ad}(G)$ coincide if and only if the number $\pi i$ is not representable as a linear combination of the numbers $\lambda_{k}$ with rational coefficients.

In distinction from the nilpotent case, criteria for the existence of a lattice in connected and simply-connected solvable Lie groups have rather cumbersome formulations. The criterion that we present is due to Auslander [2] and makes use of the concept of semisimple splitting.

Let $G$ be a connected and simply-connected Lie group. We call a connected and simply-connected solvable Lie group $G_{s}=T \ltimes_{\nu_{s}} N_{s}$ a semisimple splitting for $G$ if the following hold:

(i) $N_{s}$ is the nilradical of $G_{s}$ - the so called nilshadow of $G-$ and $T \cong \mathbb{R}^{k}$ for $k=\operatorname{dim} G_{s}-\operatorname{dim} N_{s}$

(ii) $T$ acts on $N_{s}$ via $\nu_{s}$ by semisimple automorphisms,

(iii) $G$ is a closed normal subgroup of $G_{s}$ and $G_{s}=T \ltimes_{\varpi} G$,

(iv) $N_{s}=Z_{N_{s}}(T) \cdot\left(N_{s} \cap G\right)$, where $Z_{N_{s}}(T)$ denotes the centralizer of $T$ in $N_{s}$.

This definition then implies (see e.g. [13, Lemma 5.2]) that $N_{s}$ is a connected and simply-connected nilpotent Lie group, $N=N_{s} \cap G$, and $G / N \cong T$. 
Theorem 4.9 ([13, Theorems 5.3 and 5.4]). Let $G$ be a connected and simplyconnected solvable Lie group. Then $G$ admits a unique semisimple splitting.

We shall not give the whole proof of this theorem that can be found in [13]. But we shortly describe the construction of the semisimple splitting. In order to do this, we recall the Jordan decomposition of certain morphisms:

Let $\varphi$ be an endomorphism of a finite-dimensional vector space over a field of characteristic zero. There is a unique Jordan sum decomposition

$$
\varphi=\varphi_{s}+\varphi_{n}, \quad \varphi_{s} \circ \varphi_{n}=\varphi_{n} \circ \varphi_{s},
$$

with $\varphi_{s}$ semisimple and $\varphi_{n}$ nilpotent. They are called respectively the semisimple part and the nilpotent part of $\varphi$. If $\varphi$ is an automorphism, it also has a unique Jordan product decomposition

$$
\varphi=\varphi_{s} \circ \varphi_{u}, \quad \varphi_{s} \circ \varphi_{u}=\varphi_{u} \circ \varphi_{s}
$$

with $\varphi_{s}$ semisimple and $\varphi_{u}$ unipotent; $\varphi_{s}$ is the same as in the sum decomposition and $\varphi_{u}=\mathrm{id}+\left(\varphi_{s}^{-1} \circ \varphi_{n}\right)$. The latter is called the unipotent part of $\varphi$.

Note, if $\varphi$ is a derivation resp. an automorphism of a Lie algebra, then the semisimple and the nilpotent resp. unipotent part of $\varphi$ are also derivations resp. automorphisms of the Lie algebra.

Now, let $G$ be a connected and simply-connected Lie group and $f: G \rightarrow G$ a Lie group automorphism.

Then $f_{*}:=d_{e} f$ is a Lie algebra automorphism which has a Jordan product decomposition $f_{*}=\left(f_{*}\right)_{s} \circ\left(f_{*}\right)_{u}=\left(f_{*}\right)_{u} \circ\left(f_{*}\right)_{s}$. The semisimple and unipotent part of $f$ are by definition the unique Lie group automorphisms $f_{s}, f_{u}: G \rightarrow G$ with $d_{e} f_{s}=\left(f_{*}\right)_{s}$ and $d_{e} f_{u}=\left(f_{*}\right)_{u}$.

Construction of the semisimple splitting. Let $G$ be a connected and simplyconnected solvable Lie group. Denote by $N$ the nilradical of $G$.

By [13, Proposition 3.3], there exists a connected and simply-connected nilpotent Lie subgroup $H$ of $G$ such that $G=H \cdot N$. Fix such an $H$ and consider the well-defined (!) action $\widetilde{\varpi}: H \rightarrow \mathrm{A}(G)$ given by $\widetilde{\varpi}(a)(h \cdot n):=h \cdot\left(\left.I_{a}\right|_{N}\right)_{s}(n)$, where $\left(\left.I_{a}\right|_{N}\right)_{s}$ is the semisimple part of the automorphism of $N$ which is obtained by conjugating every element of $N$ by $a$.

Define $T:=H /(N \cap H) \cong H N / N \cong G / N \cong \mathbb{R}^{k}$. Note that there is an action $\varpi$ of $T$ on $G$ making the following diagram commutative:

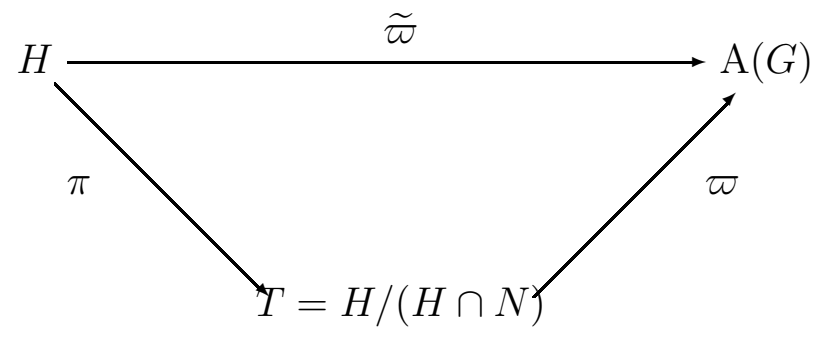


Set $G_{s}:=T \ltimes_{\varpi} G$.

One calculates that

$$
N_{s}:=\left\{\pi\left(h^{-1}\right) \cdot h \mid h \in H\right\} \cdot N=\left\{\left(\pi\left(h^{-1}\right), h \cdot n\right) \mid h \in H, n \in N\right\} \subset T \ltimes G=G_{s}
$$

is the nilradical of $G_{s}$. Furthermore, we have $T \cdot N_{s}=G_{s}$ and $T \cap N_{s}=\{e\}$. For $t \in T, h \in H, n \in N$ and every $h_{t} \in \pi^{-1}(\{t\})$ holds

$$
\begin{aligned}
\nu_{s}(t)\left(\pi\left(h^{-1}\right) \cdot(h \cdot n)\right) & :=t \cdot \pi\left(h^{-1}\right) \cdot(h \cdot n) \cdot t^{-1} \\
& =\pi\left(h^{-1}\right) \cdot\left(h \cdot \widetilde{\varpi}\left(h_{t}\right)(n)\right),
\end{aligned}
$$

i.e. $\nu_{s}(t)$ is a semisimple automorphism and $G_{s}=T \ltimes_{\nu_{s}} N_{s}$.

Remark. As usual, we denote the Lie algebras of the above Lie groups by the corresponding small German letters. In [18, Chapter III] can be found:

There exists a vector subspace $V$ of $|\mathfrak{g}|$ with $|\mathfrak{g}|=V \oplus|\mathfrak{n}|$ as vector spaces and $\forall_{A, B \in V} \operatorname{ad}(A)_{s}(B)=0$, where $\operatorname{ad}(A)_{s}$ denotes the semisimple part of $\operatorname{ad}(A)$.

Let $\mathfrak{v}$ be a copy of $V$, considered as abelian Lie algebra. Then the Lie algebra of the semisimple splitting for $G$ is $\mathfrak{g}_{s}=\mathfrak{v} \ltimes_{a d(\ldots)_{s}} \mathfrak{g}$, i.e.

$$
\forall(A, X),(B, Y) \in \mathfrak{g}_{s}[(A, X),(B, Y)]=\left(0,[X, Y]+\operatorname{ad}(A)_{s}(Y)-\operatorname{ad}(B)_{s}(X)\right),
$$

with nilradical $\mathfrak{n}_{s}=\left\{\left(-X_{V}, X\right) \mid X \in \mathfrak{g}\right\}$, where $X_{V}$ denotes the component of $X$ in $V$.

Now we state the announced criterion for the existence of lattices in solvable Lie groups.

Theorem 4.10 ([2, p. 248]). Let $G$ be a connected and simply-connected solvable Lie group with nilradical $N$ and semisimple splitting $G_{s}=T \ltimes_{\nu_{s}} N_{s}$, where $N_{s}$ is the nilshadow of $G$.

Then $G / N$ is contained as a subgroup in $G_{s} / N=T \times\left(N_{s} / N\right)$ and the projections $\pi_{1}: G / N \rightarrow T, \pi_{2}: G / N \rightarrow N_{s} / N$ are isomorphisms of abelian Lie groups.

Moreover, $G$ admits a lattice if and only if the following conditions are satisfied:

(i) There exists a basis $\mathfrak{X}:=\left\{X_{1}, \ldots, X_{n}, \ldots, X_{m}\right\}$ with rational structure constants of the Lie algebra $\mathfrak{n}_{s}$ of $N_{s}$ such that $\left\{X_{1}, \ldots, X_{n}\right\}$ is a basis of the Lie algebra $\mathfrak{n}$ of $N$.

We write $\mathfrak{n}_{s}(\mathbb{Q})$ for the rational Lie algebra $\langle\mathfrak{X}\rangle_{\mathbb{Q}}$ and $N_{s}(\mathbb{Q})$ for its image under the exponential map.

(ii) There exists a lattice subgroup $\Gamma_{T}$ of $T$ with $\Gamma_{T} \subset \pi_{1} \circ \pi_{2}^{-1}\left(N_{s}(\mathbb{Q}) / N\right)$ such that the natural action $\Gamma_{T} \rightarrow \mathrm{A}\left(\mathfrak{n}_{s}(\mathbb{Q})\right)$ is described by integer matrices in an appropriate basis of $\mathfrak{n}_{s}(\mathbb{Q})$. 


\section{Three-dimensional solvmanifolds}

The only one- and two-dimensional solvmanifolds are tori. Therefore, we begin our studies of low-dimensional solvmanifolds in dimension three.

Proposition 5.1 ([3]). Every 3-dimensional connected and simply-connected solvable non-nilpotent Lie group $G$ that possesses a lattice $\Gamma$ has a 2-dimensional nilradical. The Lie group can be written as $G=\mathbb{R} \ltimes_{\mu} \mathbb{R}^{2}$ and the lattice as $\Gamma=\mathbb{Z} \ltimes_{\mu} \mathbb{Z}^{2}$

Proof. This is a direct consequence of Proposition 3.5 and Corollary 4.5.

Theorem 5.2. A three-dimensional solvmanifold $G / \Gamma$ is non-formal if and only if $b_{1}(G / \Gamma)=2$. In this case, $G / \Gamma$ is diffeomorphic to a nilmanifold.

Proof. By Theorem 2.7, it suffices to consider the case when $G$ is solvable and non-nilpotent. The last proposition implies that there is a map $\nu: \mathbb{Z} \rightarrow \operatorname{SL}(2, \mathbb{Z})$ such that $\Gamma=\mathbb{Z} \ltimes_{\nu} \mathbb{Z}^{2}$.

If none of the roots of $\nu(1)$ equals 1 , Proposition 4.7 implies $b_{1}=1$, so $G / \Gamma$ is formal by Theorem 1.1.10.

Assume that $\nu(1)$ possesses the double root 1 . Then Proposition 4.7 implies $b_{1}=3$ if $\nu(1)$ is diagonalisable and $b_{1}=2$ if $\nu(1)$ is not diagonalisable.

Case A: $\nu(1)$ is diagonalisable

Recall that a solvmanifold is uniquely determined by its fundamental group. Therefore, we can assume $G=\mathbb{R} \ltimes_{\mu} \mathbb{R}^{2}$ and $\Gamma=\mathbb{Z} \ltimes_{\mu}\left\langle v_{1}, v_{2}\right\rangle_{\mathbb{Z}}$ with linearly independent $v_{1}, v_{2} \in \mathbb{R}^{2}$ and $\mu(t) \equiv \mathrm{id}$. In this case, $G / \Gamma$ is a torus which is formal.

Case B: $\nu(1)$ is not diagonalisable

In this case, we can assume $G=\mathbb{R} \ltimes_{\mu} \mathbb{R}^{2}$ as well as $\Gamma=\mathbb{Z} \ltimes_{\mu}\left\langle v_{1}, v_{2}\right\rangle_{\mathbb{Z}}$ with linearly independent $v_{1}, v_{2} \in \mathbb{R}^{2}$ and

$$
\mu(t)=\left(\begin{array}{ll}
1 & t \\
0 & 1
\end{array}\right)
$$

The Lie algebra $\mathfrak{g}=\langle T, X, Y \mid[T, Y]=X\rangle$ of $G$ is nilpotent, so $G / \Gamma$ is a nilmanifold with $b_{1}=2$. Therefore, it cannot be a torus and is not formal by Theorem 2.7.

In [3, Chapter III §3] the three-dimensional solvmanifolds which have no nilmanifold structure are examined. This, together with the last theorem, yields a "cohomological" classification of three-dimensional solvmanifolds.

Theorem 5.3. Every 3-dimensional solvmanifold $G / \Gamma$ is contained in Table 1 . In particular, $G / \Gamma$ is non-formal if and only if it is a non-toral nilmanifold. 
Table 1: 3-dimensional solvmanifolds

\begin{tabular}{|c|c|c|c|c|}
\hline \hline & $b_{1}(G / \Gamma)$ & $G / \Gamma$ formal & Nilmfd. $^{3}$ & c.s. $^{4}$ \\
\hline \hline a) & 3 & yes & Torus & yes \\
\hline b) & 2 & no & yes & yes \\
\hline c) & 1 & yes & no & yes \\
\hline d) & 1 & yes & no & no \\
\hline
\end{tabular}

Example. The torus $\mathbb{R}^{3} / \mathbb{Z}^{3}$ is a solvmanifold with $b_{1}=3$, and examples of 3-dimensional solvmanifolds with $b_{1}=2$ will be given in the next theorem.

For $i \in\{1,2\}$ consider the Lie groups $G_{i}=\mathbb{R} \ltimes_{\mu_{i}} \mathbb{R}^{2}$, where $\mu_{i}$ is given by $\mu_{1}(t)(x, y)=\left(e^{t} x, e^{-t} y\right), \mu_{2}(t)(x, y)=(\cos (t) x+\sin (t) y,-\sin (t) x+\cos (t) y)$.

$G_{1}$ is completely solvable and possesses the lattice

$$
\Gamma_{1}:=t_{1} \mathbb{Z} \ltimes_{\mu_{1}}\left\langle\left(\begin{array}{c}
1 \\
1
\end{array}\right),\left(\begin{array}{c}
\frac{18+8 \sqrt{5}}{7+3 \sqrt{5}} \\
\frac{2}{3+\sqrt{5}}
\end{array}\right)\right\rangle_{\mathbb{Z}},
$$

where $t_{1}=\ln \left(\frac{3+\sqrt{5}}{2}\right)$. Note that the following equation implies that $\Gamma_{1}$ really is a lattice

$$
\left(\begin{array}{cc}
e^{t_{1}} & 0 \\
0 & e^{-t_{1}}
\end{array}\right)=\left(\begin{array}{cc}
1 & \frac{18+8 \sqrt{5}}{7+3 \sqrt{5}} \\
1 & \frac{2}{3+\sqrt{5}}
\end{array}\right)\left(\begin{array}{cc}
0 & -1 \\
1 & 3
\end{array}\right)\left(\begin{array}{cc}
1 & \frac{18+8 \sqrt{5}}{7+3 \sqrt{5}} \\
1 & \frac{2}{3+\sqrt{5}}
\end{array}\right)^{-1}
$$

$G_{2}$ is not completely solvable and contains the lattice

$$
\Gamma_{2}=\pi \mathbb{Z} \ltimes_{\mu_{2}} \mathbb{Z}^{2}
$$

A short computation yields that the abelianisations of $\Gamma_{i}$ have rank one, i.e. we have constructed examples of type c) and d) in Table 1.

Theorem 5.4. Every lattice in the unique 3-dimensional connected and simplyconnected non-abelian nilpotent Lie group

$$
U_{3}(\mathbb{R}):=\left\{\left(\begin{array}{ccc}
1 & x & z \\
0 & 1 & y \\
0 & 0 & 1
\end{array}\right) \mid x, y, z \in \mathbb{R}\right\}
$$

is isomorphic to $\Gamma_{3, n}:=\Gamma_{3, n}(\mathbb{Z}):=\left\{\left(\begin{array}{ccc}1 & x & \frac{z}{n} \\ 0 & 1 & y \\ 0 & 0 & 1\end{array}\right) \mid x, y, z \in \mathbb{Z}\right\}$ with $n \in \mathbb{N}_{+}$.

Therefore, any three-dimensional nilmanifold with $b_{1}=2$ is of the form $U_{3}(\mathbb{R}) / \Gamma_{3, n}(\mathbb{Z})$.

$\Gamma_{3, n}(\mathbb{Z})$ is presented by $\left\langle e_{1}, e_{2}, e_{3}\right|\left[e_{1}, e_{2}\right]=e_{3}^{n}$ and $e_{3}$ central $\rangle$.

\footnotetext{
${ }^{3}$ possesses the structure of a solvmanifold as quotient of a nilpotent Lie group

${ }^{4}$ possesses the structure of a solvmanifold as quotient of a completely solvable Lie group
} 
Proof. $U_{3}(\mathbb{R})$ is the only connected and simply-connected non-abelian nilpotent Lie group of dimension three. By [3, Chapter III $\S 7$ ], each lattice in it is isomorphic to $\Gamma_{3, n}$. The other assertions follow trivially.

Sometimes, we shall write $(x, y, z)$ for the corresponding matrix in $U_{3}(\mathbb{R})$.

For later applications, we are going to determine the Lie group automorphisms and the one-parameter groups of $U_{3}(\mathbb{R})$. In order to do this, we start with the following proposition. Note that $Z(G)$ denotes the center of a group $G$.

\section{Proposition 5.5.}

(i) $\left[U_{3}(\mathbb{R}), U_{3}(\mathbb{R})\right]=Z\left(U_{3}(\mathbb{R})\right)=\{(0,0, z) \mid z \in \mathbb{R}\}, U_{3}(\mathbb{R}) / Z\left(U_{3}(\mathbb{R})\right) \cong \mathbb{R}^{2}$

(ii) Every Lie group homomorphism $f: U_{3}(\mathbb{R}) \rightarrow U_{3}(\mathbb{R})$ induces natural Lie group homomorphisms

$$
f_{Z}: Z\left(U_{3}(\mathbb{R})\right) \longrightarrow Z\left(U_{3}(\mathbb{R})\right)
$$

and

$$
\begin{array}{rlc}
\bar{f}: \quad U_{3}(\mathbb{R}) / Z\left(U_{3}(\mathbb{R})\right) & \longrightarrow & U_{3}(\mathbb{R}) / Z\left(U_{3}(\mathbb{R})\right) . \\
{[(x, y, 0)]=[(x, y, z)]} & \longmapsto & {[f((x, y, z))]=\left[\left(f_{1}(x, y, 0), f_{2}(x, y, 0), 0\right)\right]}
\end{array}
$$

$\bar{f}$ uniquely determines $f_{Z}$, and $\bar{f}$ is an automorphism if and only if $f$ is such.

(iii) Let $\gamma_{1}=\left(a_{1}, b_{1}, \frac{c_{1}}{n}\right), \gamma_{2}=\left(a_{2}, b_{2}, \frac{c_{2}}{n}\right) \in \Gamma_{3, n}$. Then there is a unique homomorphism $g: \Gamma_{3, n} \rightarrow \Gamma_{3, n}$ such that $g((1,0,0))=\gamma_{1}$ and $g((0,1,0))=\gamma_{2}$. Moreover, $g\left(\left(0,0, \frac{1}{n}\right)\right)=\left(0,0, \frac{1}{n}\left(a_{1} b_{2}-a_{2} b_{1}\right)\right)$.

One has $\Gamma_{3, n} / Z\left(\Gamma_{3, n}\right) \cong \mathbb{Z}^{2}$, and $g$ is an isomorphism if and only if

$$
\bar{g}: \Gamma_{3, n} / Z\left(\Gamma_{3, n}\right) \longrightarrow \Gamma_{3, n} / Z\left(\Gamma_{3, n}\right)
$$

is an isomorphism, i.e. $a_{1} b_{2}-a_{2} b_{1}= \pm 1$.

Proof. (i) is trivial.

ad (ii): Let $f: U_{3}(\mathbb{R}) \rightarrow U_{3}(\mathbb{R})$ be a Lie group homomorphism. Then

$$
f((0,0, z))=[f((z, 0,0)), f((0,1,0))] \in Z\left(U_{3}(\mathbb{R})\right),
$$

i.e. $f\left(Z\left(U_{3}(\mathbb{R})\right)\right) \subset Z\left(U_{3}(\mathbb{R})\right)$. Moreover, one has for $(a, b, c):=f((x, y, 0))$

$$
(a, b, 0)^{-1} \cdot(a, b, c)=(-a,-b, 0) \cdot(a, b, c)=(0,0,-a b+c) \in Z\left(U_{3}(\mathbb{R})\right),
$$

and therefore $[(a, b, 0)]=\bar{f}([(x, y, 0)])$. Now, (5) implies that $f_{Z}$ is uniquely determined by $\bar{f}$. 
Assume, $f$ is an isomorphism. Then (5) also holds for $f^{-1}$ and we get $f\left(Z\left(U_{3}(\mathbb{R})\right)\right)=Z\left(U_{3}(\mathbb{R})\right)$, i.e. $f_{Z}$ is an isomorphism of the additive group $\mathbb{R}$. Since $f$ is continuous, there exists $m \in \mathbb{R} \backslash\{0\}$ such that $f_{Z}((0,0, z))=(0,0, m z)$. Denote by $\left(f_{i j}\right)_{1 \leq i, j \leq 2}$ the matrix of $\bar{f}: \mathbb{R}^{2} \rightarrow \mathbb{R}^{2}$ with respect to the basis $\left\{\left(\begin{array}{l}1 \\ 0\end{array}\right),\left(\begin{array}{l}0 \\ 1\end{array}\right)\right\}$ of the vector space $\mathbb{R}^{2}$. One calculates

$$
\begin{aligned}
\left(0,0, \operatorname{det}\left(f_{i j}\right)\right)= & {\left[\left(f_{11}, f_{21}, 0\right),\left(f_{12}, f_{22}, 0\right)\right]=[f((1,0,0)), f((0,1,0))] } \\
& \stackrel{\underline{\underline{5}}}{=}(0,0, m),
\end{aligned}
$$

so $\bar{f}$ is an automorphism, since $m \neq 0$.

Conversely, if $\bar{f}$ is an automorphism, then the homomorphism $f_{Z}$ is given by $f_{Z}((0,0, z))=(0,0, \operatorname{det}(\bar{f}) z)$ which is even an automorphism. Therefore, the 5 -Lemma implies that $f$ is an automorphism.

ad (iii): Let $\gamma_{1}, \gamma_{2}$ be as in (iii). Then $\left[\gamma_{1}, \gamma_{2}\right]=\left(0,0, \frac{1}{n}\left(a_{1} b_{2}-a_{2} b_{1}\right)\right)^{n}$ and this implies the existence of the (unique) homomorphism $g$ with the mentioned properties.

If $g$ is an isomorphism, then $g\left(Z\left(\Gamma_{3, n}\right)\right)=Z\left(\Gamma_{3, n}\right)=\left\{\left(0,0, \frac{z}{n}\right) \mid z \in \mathbb{Z}\right\}$, and therefore $\left|a_{1} b_{2}-a_{2} b_{1}\right|=1$. Since the matrix of $\bar{g}$ has determinant $a_{1} b_{2}-a_{2} b_{1}, \bar{f}$ is an isomorphism.

Again, the converse is trivial.

Theorem 5.6. As a set, the group of Lie group automorphisms $\mathrm{A}\left(U_{3}(\mathbb{R})\right)$ equals $\mathrm{GL}(2, \mathbb{R}) \times \mathbb{R}^{2}$, the group law is given by

$$
\left.(A, a) \circ(B, b) \longmapsto\left(A B, \operatorname{det}(B) B^{-1} a+\operatorname{det}(A) b\right)\right),
$$

and for $f=\left(A=\left(\begin{array}{ll}\alpha & \beta \\ \gamma & \delta\end{array}\right),\left(\begin{array}{l}u \\ v\end{array}\right)\right) \in \mathrm{A}\left(U_{3}(\mathbb{R})\right)$ and $(x, y, z) \in U_{3}(\mathbb{R})$ we have

$$
\begin{aligned}
f((x, y, z))= & (\alpha x+\beta y, \gamma x+\delta y, \\
& \left.\operatorname{det}(A) z+\beta \gamma x y+\frac{\alpha \gamma}{2} x^{2}+\frac{\beta \delta}{2} y^{2}+u y-v x\right) .
\end{aligned}
$$

Proof. Let $f \in \mathrm{A}\left(U_{3}(\mathbb{R})\right)$ and $(x, y, z) \in U_{3}(\mathbb{R})$ be given. We have to show that there is $\left(\left(\begin{array}{ll}\alpha & \beta \\ \gamma & \delta\end{array}\right),\left(\begin{array}{l}u \\ v\end{array}\right)\right) \in \mathrm{GL}(2, \mathbb{R}) \times \mathbb{R}^{2}$ such that $f((x, y, z))$ satisfies (7). Then a short computation yields (6).

Let $\left(\begin{array}{cc}\alpha & \beta \\ \gamma & \delta\end{array}\right) \in \operatorname{GL}(2, \mathbb{R})$ be the matrix of $\bar{f}$ with respect to the canonical basis of $\mathbb{R}^{2}$. We showed in the last proof $f((0,0, z))=(0,0 \operatorname{det}(\bar{f}) z)$.

There exist smooth functions $f_{1}, f_{2}: \mathbb{R} \rightarrow \mathbb{R}$ with

$$
f((x, 0,0))=\left(\alpha x, \gamma x, f_{1}(x)\right)
$$




$$
f((0, y, 0))=\left(\beta y, \delta y, f_{2}(y)\right)
$$

We set $u:=f_{2}^{\prime}(0)$ and $v:=-f_{1}^{\prime}(0)$. The homomorphism property of $f$ implies

$$
\begin{aligned}
\frac{1}{h}\left(f_{1}(x+h)-f_{1}(x)\right) & =\frac{f_{1}(x)-f_{1}(0)}{h}+\alpha \gamma x, \\
\frac{1}{h}\left(f_{2}(y+h)-f_{2}(y)\right) & =\frac{f_{2}(y)-f_{2}(0)}{h}+\beta \delta y,
\end{aligned}
$$

and this yields

$$
\begin{aligned}
& f_{1}(x)=-v x+\frac{\alpha \gamma}{2} x^{2} \\
& f_{2}(y)=u y+\frac{\beta \delta}{2} y^{2} .
\end{aligned}
$$

Using $(x, y, z)=(0, y, 0)(x, 0,0)(0,0, z)$, one computes (7).

Corollary 5.7. $f=\left(A,\left(\begin{array}{l}u \\ v\end{array}\right)\right) \in \mathrm{A}\left(U_{3}(\mathbb{R})\right)$ with $A=\left(\begin{array}{cc}\alpha & \beta \\ \gamma & \delta\end{array}\right)$ lies on a oneparameter group of $\mathrm{A}\left(U_{3}(\mathbb{R})\right)$ if and only if $A$ lies one a one-parameter group of $G L(2, \mathbb{R})$.

$$
\text { If } \nu_{t}=\left(\begin{array}{cc}
\alpha_{t} & \beta_{t} \\
\gamma_{t} & \delta_{t}
\end{array}\right) \text { denotes a one-parameter group with } \nu_{1}=A \text {, then the map }
$$

$\mu_{t}: \mathbb{R} \rightarrow \mathrm{A}\left(U_{3}(\mathbb{R})\right)$ defined by

$$
\begin{aligned}
& \mu_{t}((x, y, z))=\left(\alpha_{t} x+\beta_{t} y, \gamma_{t} x+\delta_{t} y,\right. \\
& \underbrace{\left(\alpha_{t} \delta_{t}-\beta_{t} \delta_{t}\right)}_{=1} z+\beta_{t} \gamma_{t} x y+\frac{\alpha_{t} \gamma_{t}}{2} x^{2}+\frac{\beta_{t} \delta_{t}}{2} y^{2}+t u y-t v x)
\end{aligned}
$$

is a one-parameter group with $\mu_{1}=f$.

Proof. The only claim that is not obvious is the fact that $\mu_{t}$ defines a oneparameter group. Using $\nu_{t+s}=\nu_{t} \circ \nu_{s}$, this can be seen by a short calculation.

\section{Four-dimensional solvmanifolds}

As we have done in the three-dimensional case, we are going to give a "cohomological" classification of four-dimensional solvmanifolds. We shall consider all isomorphism classes of lattices that can arise in a four-dimensional connected and simply-connected solvable Lie group. The next proposition describes such lattices in the case of a non-nilpotent group.

Proposition 6.1. Every 4-dimensional connected and simply-connected solvable non-nilpotent Lie group $G$ that possesses a lattice $\Gamma$ has a 3-dimensional nilradical $N$ which is either $\mathbb{R}^{3}$ or $U_{3}(\mathbb{R})$. Therefore, $G / \Gamma$ fibers over $S^{1}$ (this is the Mostow 
bundle) and the Lie group can be written as $G=\mathbb{R} \ltimes_{\mu} N$. If $N$ is abelian, a basis transformation yields $\Gamma=\mathbb{Z} \ltimes_{\mu_{\mathbb{Z}^{3}}} \mathbb{Z}^{3}$. Otherwise, $\Gamma$ is isomorphic to $\mathbb{Z} \ltimes_{\nu} \Gamma_{3, n}$, where $\nu: \mathbb{Z} \rightarrow \operatorname{Aut}\left(\Gamma_{3, n}\right)$ is a group homomorphism with

$$
\begin{aligned}
\nu(1)\left(x, y, \frac{z}{n}\right)=\left(a_{1} x+a_{2} y, \quad b_{1} x+b_{2} y,\right. & a_{2} b_{1} x y+a_{1} b_{1} \frac{x(x-1)}{2}+a_{2} b_{2} \frac{y(y-1)}{2} \\
& +\frac{1}{n}\left(c_{1} x+c_{2} y+\left(a_{1} b_{2}-a_{2} b_{1}\right) z\right)
\end{aligned},
$$

where $c_{1}, c_{2}, \in \mathbb{Z}$, and $\left(\begin{array}{ll}a_{1} & a_{2} \\ b_{1} & b_{2}\end{array}\right) \in \mathrm{GL}(2, \mathbb{Z})$ is the matrix of $\overline{\nu(1)}$ with respect to the canonical basis of the $\mathbb{Z}$-module $\mathbb{Z}^{2}=\Gamma_{3, n} / Z\left(\Gamma_{3, n}\right)$. Moreover, $\bar{\nu}(1)$ lies on a one-parameter group $\mathbb{R} \rightarrow \mathrm{A}\left(U_{3}(\mathbb{R}) / Z\left(U_{3}(\mathbb{R})\right)\right)=\mathrm{GL}(2, \mathbb{R})$, i.e. $\bar{\nu}(1) \in \mathrm{SL}(2, \mathbb{R})$.

Proof. From [48, Theorem 3.1.10] follows $\operatorname{dim} N=3$ and $G=\mathbb{R} \ltimes_{\mu} N$. If $N$ is abelian, Corollary 4.5 implies that we can assume $\Gamma=\mathbb{Z} \ltimes_{\left.\mu\right|_{\mathbb{Z}^{3}}} \mathbb{Z}^{3}$.

Assume now that $N$ is not abelian, i.e. $N=U_{3}(\mathbb{R}) . \Gamma_{N}=\Gamma \cap N$ is a lattice in $N$ and by Theorem [5.4, we have $\Gamma_{N}=\Gamma_{3, n}$. By Corollary 4.5, there is a homomorphism $\nu: \mathbb{Z} \rightarrow \operatorname{Aut}\left(\Gamma_{3, n}\right)$ with $\Gamma \cong \mathbb{Z} \ltimes_{\nu} \Gamma_{3, n}$. Proposition 5.5(iii) implies that $\nu(1)$ is determined by $\left(a_{1}, b_{1}, \frac{c_{1}}{n}\right):=\nu(1)((1,0,0))$ and $\left(a_{2}, b_{2}, \frac{c_{2}}{n}\right):=$ $\nu(1)((0,1,0)) \in \Gamma_{3, n}$. Since $\left(x, y, \frac{z}{n}\right)=(0,1,0)^{y}(1,0,0)^{x}\left(0,0, \frac{1}{n}\right)^{z}$, a short computation yields the claimed formula for $\nu(1)\left(\left(x, y, \frac{z}{n}\right)\right)$.

Further, Corollary 5.7 implies that $\bar{\nu}(1)$ lies on a one-parameter group.

Theorem 6.2. Every 4-dimensional solvmanifold $G / \Gamma$ is contained in Table Q In particular, $G / \Gamma$ is non-formal if and only if it is a non-toral nilmanifold.

Table 2: 4-dimensional solvmanifolds

\begin{tabular}{|c|c|c|c|c|c|c|c|}
\hline \hline & $b_{1}(G / \Gamma)$ & $G / \Gamma$ formal & symplectic & complex & Kähler & Nilmfd. ${ }^{5}$ & c.s. $^{6}$ \\
\hline \hline a) & 4 & yes & yes & Torus & yes & Torus & yes \\
\hline b) & 3 & no & yes & PKS $^{7}$ & no & yes & yes \\
\hline c) & 2 & yes & yes & no & no & no & yes \\
\hline d) & 2 & yes & yes & HS $^{8}$ & yes & no & no \\
\hline e) & 2 & no & yes & no & no & yes & yes \\
\hline f) & 1 & yes & no & no & no & no & yes \\
\hline g) & 1 & yes & no & IS $^{9}$ & no & no & no \\
\hline h) & 1 & yes & no & IS $^{+10}$ & no & no & yes \\
\hline i) & 1 & yes & no & SKS & no & no & no \\
\hline
\end{tabular}

\footnotetext{
${ }^{5}$ possesses the structure of a solvmanifold as quotient of a nilpotent Lie group

${ }^{6}$ possesses the structure of a solvmanifold as quotient of a completely solvable Lie group

${ }^{7}$ PrimaryKodaira Surface

${ }^{8}$ Hyperelliptic Surface

${ }^{9}$ Inoue Surface of Type $\mathbf{S}^{\mathbf{0}}$

${ }^{10}$ Inoue Surface of Type $\mathbf{S}^{+}$

${ }^{11}$ Secondary Kodaira Surface
} 
Proof. Apart from the column on formality the theorem follows from works of Geiges [24] and Hasegawa [30]. (Attention: In [30] a more general notion of solvmanifold is used!)

A decomposable four-dimensional connected and simply-connected nilpotent Lie group is abelian or has a two-dimensional center. The only connected and simply-connected indecomposable nilpotent Lie group of dimension four has a two-dimensional commutator. By Propositions 2.4 and 2.3, the corresponding nilmanifolds have the structure of orientable $T^{2}$-bundles over $T^{2}$. (The orientability follows from the total spaces' orientability.)

From a result of Geiges [24, Theorems 1 and 3] follows that they are contained in Table 2, (Recall that a nilmanifold is formal if and only if it is a torus.) In particular, every four-dimensional nilmanifold is symplectic.

Now, we regard a lattice $\Gamma=\mathbb{Z} \ltimes_{\nu} \Gamma_{N}, \Gamma_{N} \in\left\{\mathbb{Z}^{3}, \Gamma_{3, n}(\mathbb{Z})\right\}$, in a Lie group $G=\mathbb{R} \ltimes_{\mu} N$ as in the last proposition.

We expand Hasegawa's argumentation in [30] by the aspect of formality and consider the "roots" of $\nu(1)$. Recall, Corollary 3.2 implies that a solvmanifold is determined by its fundamental group. Below, we shall use this fact several times.

Case A.: $\Gamma_{N}=\mathbb{Z}^{3}$

By Proposition 6.1, $\nu$ extends to a one-parameter group $\mathbb{R} \rightarrow \mathrm{SL}(3, \mathbb{R})$. Denote by $\lambda_{1}, \lambda_{2}, \lambda_{3} \in \mathbb{C}$ the roots of $\nu(1) \in \mathrm{SL}(3, \mathbb{Z})$, i.e. $\lambda_{1} \cdot \lambda_{2} \cdot \lambda_{3}=1$. We get from Theorem 4.3 and Lemma B.4 that the following subcases can occur:

A.1.) $\lambda_{1}, \lambda_{2}, \lambda_{3} \in \mathbb{R}_{+}$

A.1.1.) $\exists_{i_{0}} \lambda_{i_{0}}=1$ (w.l.o.g. $\lambda_{1}=1$ )

A.1.1.1.) $\lambda_{2}=\lambda_{3}=1$

A.1.1.2.) $\lambda_{2}=\lambda_{3}^{-1} \in \mathbb{R} \backslash\{1\}$

A.1.2.) $\forall_{i} \lambda_{i} \neq 1$

A.1.2.1.) $\nu(1)$ is diagonalisable

A.1.2.2.) $\nu(1)$ is not diagonalisable

A.2.) $\lambda_{1}=1, \lambda_{2}=\lambda_{3}=-1$ and $\nu(1)$ is diagonalisable

A.3.) $\exists_{i_{0}} \lambda_{i_{0}} \in \mathbb{C} \backslash \mathbb{R}$ (w.l.o.g. $\lambda_{2}=\overline{\lambda_{3}} \in \mathbb{C} \backslash \mathbb{R}$ and $\lambda_{1} \in \mathbb{R}_{+}$)

A.3.1.) $\lambda_{1}=1$

A.3.2.) $\lambda_{1} \neq 1$

Case A.1.1.1.: $\lambda_{1}=\lambda_{2}=\lambda_{3}=1$

If $\nu(1)$ is diagonalisable, then $G / \Gamma$ is clearly a torus. This is case a). If $\nu(1)$ is not diagonalisable we can assume $G=\mathbb{R} \ltimes_{\mu} \mathbb{R}^{3}$ and $\Gamma=\mathbb{Z} \ltimes_{\mu}\left\langle v_{1}, v_{2}, v_{3}\right\rangle_{\mathbb{Z}}$, where 
$\mu(t)$ is one of the following one-parameter groups

$$
\begin{aligned}
\exp \left(t\left(\begin{array}{lll}
0 & 0 & 0 \\
0 & 0 & 1 \\
0 & 0 & 0
\end{array}\right)\right) & =\left(\begin{array}{ccc}
1 & 0 & 0 \\
0 & 1 & t \\
0 & 0 & 1
\end{array}\right) \\
\exp \left(t\left(\begin{array}{ccc}
0 & 1 & -\frac{1}{2} \\
0 & 0 & 1 \\
0 & 0 & 0
\end{array}\right)\right) & =\left(\begin{array}{ccc}
1 & t & \frac{1}{2}\left(t^{2}-t\right) \\
0 & 1 & t \\
0 & 0 & 1
\end{array}\right) .
\end{aligned}
$$

In both cases $G / \Gamma$ is a 4 -dimensional nilmanifold and therefore symplectic. In the first case, we have a primary Kodaira surface with $b_{1}=3$, see [30, Section 2.2.3)]; in the second case the nilmanifold has $b_{1}=2$ and no complex structure, see [31, Example 2]. Being non-toral nilmanifolds, both are not formal and we get the cases b) and e).

Cases A.1.1.2. and A.1.2.1.: The $\lambda_{i}$ are positive and pairwise different or two of them are equal but $\nu(1)$ is diagonalisable. (The latter cannot happen by Lemma B.4.)

We can assume $G=\mathbb{R} \ltimes_{\mu} \mathbb{R}^{3}$ and $\Gamma=\mathbb{Z} \ltimes_{\mu}\left\langle v_{1}, v_{2}, v_{3}\right\rangle_{\mathbb{Z}}$ with linearly independent $v_{1}, v_{2}, v_{3} \in \mathbb{R}^{3}$, where $\mu(t)=\left(\begin{array}{ccc}\exp \left(t \ln \left(\lambda_{1}\right)\right) & 0 & 0 \\ 0 & \exp \left(t \ln \left(\lambda_{2}\right)\right) & 0 \\ 0 & 0 & \exp \left(t \ln \left(\lambda_{3}\right)\right)\end{array}\right)$. By

[31, Example 2], the solvmanifold $G / \Gamma$ does not admit a complex structure.

One computes the Lie algebra of $G$ as

$$
\mathfrak{g}=\left\langle T, X, Y, Z \mid[T, X]=\ln \left(\lambda_{1}\right) X,[T, Y]=\ln \left(\lambda_{2}\right) Y,[T, Z]=\ln \left(\lambda_{3}\right) Z\right\rangle
$$

which is completely solvable and non-nilpotent. Therefore, the minimal model of the Chevalley-Eilenberg complex is the minimal model of $G / \Gamma$.

If none of the roots $\lambda_{i}$ is one, we see by Proposition 4.7 that $b_{1}(G / \Gamma)=1$. Since $G / \Gamma$ is parallelisable, this implies $b_{2}(G / \Gamma)=0$, so the space cannot be symplectic. Further it is formal by Theorem 1.1.10. This is case f) in Table 2 .

If one of the roots is one (w.l.o.g. $\lambda_{1}=1$ ), we have $b_{1}(G / \Gamma)=2$ and the Chevalley-Eilenberg complex is

$$
\left(\bigwedge(\tau, \alpha, \beta, \gamma), d \tau=d \alpha=0, d \beta=-\ln \left(\lambda_{2}\right) \tau \wedge \beta, d \gamma=-\ln \left(\lambda_{3}\right) \tau \wedge \gamma\right)
$$

$\tau \wedge \alpha+\alpha \wedge \beta+\alpha \wedge \gamma-\beta \wedge \gamma$ defines a symplectic form on $G / \Gamma$. Further, one computes the first stage of the minimal model of the Chevalley-Eilenberg complex as

$$
\mathcal{M}^{\leq 1}=\bigwedge\left(x_{1}, x_{2}\right), d x_{i}=0 .
$$

Therefore, $G / \Gamma$ is 1 -formal and by Theorem 1.1.6 formal. This is case c) in Table 2 . 
Case A.1.2.2.: $\lambda_{i} \in \mathbb{R}_{+} \backslash\{1\}$ and $\nu(1)$ is not diagonalisable In this case two roots must be equal (w.l.o.g. $\lambda_{2}=\lambda_{3}$ ) and the third is different from the others, i.e. $\lambda_{1}=\frac{1}{\lambda_{2}^{2}} \neq \lambda_{2}$. Since the characteristic polynomial of $\nu(1)$ has integer coefficients, Lemma B.4 implies $\lambda_{2}= \pm 1$ and this is a contradiction.

Cases A.2. and A.3.1.: $\left.\lambda_{1}=1, \lambda_{2}=\overline{\lambda_{3}}=\exp (i \varphi) \in \mathbb{C} \backslash \mathbb{R}, \varphi \in\right] 0,2 \pi[$ We can assume $G=\mathbb{R} \ltimes_{\mu} \mathbb{R}^{3}$ and $\Gamma=\mathbb{Z} \ltimes_{\mu}\left\langle v_{1}, v_{2}, v_{3}\right\rangle_{\mathbb{Z}}$ with linearly independent $v_{1}, v_{2}, v_{3} \in \mathbb{R}^{3}$, where $\mu(t)=\left(\begin{array}{ccc}1 & 0 & 0 \\ 0 & \cos (t \varphi) & -\sin (t \varphi) \\ 0 & \sin (t \varphi) & \cos (t \varphi)\end{array}\right)$. Thus $G / \Gamma$ is a hyperelliptic surface (see [30, Section 3.3.]) which is Kählerian and has $b_{1}=2$. The Lie algebra of $G$ is not completely solvable and we are in case d).

Case A.3.2.: $\left.\lambda_{1} \neq 1, \lambda_{2}=\overline{\lambda_{3}}=\left|\lambda_{2}\right| \exp (i \varphi) \in \mathbb{C} \backslash \mathbb{R}, \varphi \in\right] 0,2 \pi[\backslash\{\pi\}$ We can assume $G=\mathbb{R} \ltimes_{\mu} \mathbb{R}^{3}$ and $\Gamma=\mathbb{Z} \ltimes_{\mu}\left\langle v_{1}, v_{2}, v_{3}\right\rangle_{\mathbb{Z}}$ with linearly independent $v_{1}, v_{2}, v_{3} \in \mathbb{R}^{3}$, where $\mu(t)=\left(\begin{array}{ccc}\lambda_{1}^{t} & 0 & 0 \\ 0 & \left|\lambda_{2}\right|^{t} \cos (t \varphi) & -\left|\lambda_{2}\right|^{t} \sin (t \varphi) \\ 0 & \left|\lambda_{2}\right|^{t} \sin (t \varphi) & \left|\lambda_{2}\right|^{t} \cos (t \varphi)\end{array}\right)$. Thus $G / \Gamma$ is a Inoue surface of type $S^{0}$ (see [30, Section 3.6.]), which is not symplectic and has $b_{1}=1$ (by Proposition 4.7, since 1 is no root of $\mu(1)$ ). By Theorem 1.1.10, $G / \Gamma$ is formal. The Lie algebra of $G$ is not completely solvable and this yields case g) of Table 2 .

Case B.: $\Gamma_{N}=\Gamma_{3, n}(\mathbb{Z})$

In this case we have a homomorphism $\nu: \mathbb{Z} \rightarrow \operatorname{Aut}\left(\Gamma_{3, n}(\mathbb{Z})\right)$. We shall write $N$ for $U_{3}(\mathbb{R})$ and $\Gamma_{N}$ for $\Gamma_{3, n}(\mathbb{Z})$. The automorphism $\nu(1)$ induces an automorphism $\bar{\nu}(1)$ of $\Gamma_{N} / Z\left(\Gamma_{N}\right)=\mathbb{Z}^{2}$ which lies by Proposition 6.1 on a one-parameter group $\mathbb{R} \rightarrow \mathrm{A}\left(U_{3}(\mathbb{R}) / \Gamma_{N}\right)=\mathrm{GL}(2, \mathbb{R})$. Denote the roots of $\bar{\nu}(1) \in \mathrm{GL}(2, \mathbb{Z})$ by $\widetilde{\lambda}_{1}, \widetilde{\lambda}_{2}$. Theorem 4.3 shows that the following cases are possible:

B.1.) $\widetilde{\lambda}_{1}, \widetilde{\lambda}_{2} \in \mathbb{R}_{+}$

$$
\begin{array}{ll}
\text { B.1.1.) } & \widetilde{\lambda}_{1}=\widetilde{\lambda}_{2}=1 \\
\text { B.1.2.) } & \widetilde{\lambda}_{1}=\widetilde{\lambda}_{2}^{-1} \neq 1
\end{array}
$$

B.2.) $\widetilde{\lambda}_{1}=\widetilde{\lambda}_{2}=-1$ and $\bar{\nu}(1)$ is diagonalisable

B.3.) $\widetilde{\lambda}_{1}=\overline{\widetilde{\lambda}_{2}} \in \mathbb{C} \backslash \mathbb{R}$

$\nu(1)$ also induces an automorphism $\nu(1)_{Z}$ of the center $Z\left(\Gamma_{N}\right)$ of $\Gamma_{N}$ which equals $\operatorname{det}(\bar{\nu}(1)) \cdot$ id $=$ id by Proposition 5.5)(iii).

Case B.1.1.: $\widetilde{\lambda}_{1}=\widetilde{\lambda}_{2}=1$

By [23, Lemma 1], we can assume $\bar{\nu}(1)=\left(\begin{array}{ll}1 & k \\ 0 & 1\end{array}\right) \in \operatorname{SL}(2, \mathbb{Z})$ with $k \in \mathbb{N}$. Then Proposition 6.1 yields $\nu(1)\left(\left(x, y, \frac{z}{n}\right)\right)=\left(x+k y, y, k \frac{y(y-1)}{2}+\frac{c_{1} x+c_{2} y+z}{n}\right)$ and this 
implies

$\Gamma=\mathbb{Z} \ltimes_{\nu} \Gamma_{3, n}=\left\langle e_{0}, \ldots, e_{3} \mid\left[e_{0}, e_{1}\right]=e_{3}^{c_{1}},\left[e_{2}^{-1}, e_{0}\right]=e_{1}^{k} e_{3}^{c_{2}},\left[e_{0}, e_{3}\right]=1,\left[e_{1}, e_{2}\right]=e_{3}^{n}\right\rangle$.

This is a discrete torsion-free nilpotent group, which can be embedded as a lattice in a connected and simply-connected nilpotent Lie group by [50, Theorem 2.18]. Since a solvmanifold is uniquely determined by its fundamental group, $G / \Gamma$ is diffeomorphic to a nilmanifold.

As at the beginning of the proof, we conclude that $G / \Gamma$ is the total space of a $T^{2}$-bundle over $T^{2}$ and occurs in our list. The quotient $G / \Gamma$ is of type b) if $k=0$ and of type e) if $k \neq 0$.

Case B.1.2.: $\widetilde{\lambda}_{1}=\widetilde{\lambda}_{2}^{-1} \in \mathbb{R}_{+} \backslash\{1\}$

We have $\bar{\nu}(1)=\left(\begin{array}{ll}a_{1} & a_{2} \\ b_{1} & b_{2}\end{array}\right) \in \mathrm{SL}(2, \mathbb{Z})$, and Proposition 6.1 implies

$$
\left.\begin{array}{rl}
\nu(1)\left(x, y, \frac{z}{n}\right)=\left(a_{1} x+a_{2} y, \quad b_{1} x+b_{2} y,\right. & a_{2} b_{1} x y+a_{1} b_{1} \frac{x(x-1)}{2}+a_{2} b_{2} \frac{y(y-1)}{2} \\
& +\frac{1}{n}\left(c_{1} x+c_{2} y+\left(a_{1} b_{2}-a_{2} b_{1}\right) z\right)
\end{array}\right)
$$

for certain $c_{1}, c_{2} \in \mathbb{Z}$.

Choose eigenvectors $\left(\begin{array}{c}v_{1} \\ v_{2}\end{array}\right),\left(\begin{array}{c}w_{1} \\ w_{2}\end{array}\right) \in \mathbb{R}^{2} \backslash\{0\}$ with respect to the eigenvalues $\widetilde{\lambda}_{1}$ resp. $\widetilde{\lambda}_{2}$ of ${ }^{\tau} \bar{\nu}(1)$ (where ${ }^{\tau}$ denotes the transpose). There exist $u_{1}, u_{2}, u_{3} \in \mathbb{R}$ such that for $\gamma_{i}:=\left(v_{i}, w_{i}, u_{i}\right), i \in\{1,2\}$, and $\gamma_{3}:=\left(0,0, u_{3}\right) \in U_{3}(\mathbb{R})$ we have

$$
\begin{gathered}
{\left[\gamma_{1}, \gamma_{2}\right]=\gamma_{3}^{n},} \\
\widetilde{\mu}(1)\left(\gamma_{1}\right)=\gamma_{1}^{a_{1}} \gamma_{2}^{b_{1}} \gamma_{3}^{c_{1}}, \widetilde{\mu}(1)\left(\gamma_{2}\right)=\gamma_{1}^{a_{2}} \gamma_{2}^{b_{2}} \gamma_{3}^{c_{2}},
\end{gathered}
$$

where $\widetilde{\mu}(t)((x, y, z))=\left(\exp \left(t \ln \left(\widetilde{\lambda}_{1}\right)\right) x, \exp \left(t \ln \left(\widetilde{\lambda}_{2}\right)\right) y, z\right)$.

Then $G / \Gamma$ is diffeomorphic to the solvmanifold $\widetilde{G} / \widetilde{\Gamma}$, where $\widetilde{G}=\mathbb{R} \ltimes_{\widetilde{\mu}} U_{3}(\mathbb{R})$ and $\widetilde{\Gamma}=\mathbb{Z} \ltimes_{\widetilde{\mu}}\left\langle\gamma_{1}, \gamma_{2}, \gamma_{3}\right\rangle$, i.e. $G / \Gamma$ is a Inoue surface of type $S^{+}$, see [30, Section 3.7]. The Lie algebra of $\widetilde{G}$,

$$
\widetilde{\mathfrak{g}}=\langle T, X, Y, Z \mid[T, X]=X,[T, Y]=-Y,[X, Y]=Z\rangle,
$$

is completely solvable and not nilpotent. Further, the knowledge of $\widetilde{\mathfrak{g}}$ implies $b_{1}(G / \Gamma)=1$. By Theorem 1.1.10, $G / \Gamma$ is formal. Therefore, this is a solvmanifold of type h) in Table 2 .

Case B.2.: $\widetilde{\lambda}_{1}=\widetilde{\lambda}_{2}=-1$ and $\bar{\nu}(1)$ is diagonalisable

[23, Lemma 1] implies that we can assume $\bar{\nu}(1)=\left(\begin{array}{cc}-1 & 0 \\ 0 & -1\end{array}\right) \in \mathrm{SL}(2, \mathbb{Z})$. So

Proposition 6.1 implies $\nu(1)\left(\left(x, y, \frac{z}{n}\right)\right)=\left(-x,-y, \frac{c_{1} x+c_{2} y+z}{n}\right)$ for certain integers $c_{1}, c_{2} \in \mathbb{Z}$. Moreover, $G / \Gamma$ is diffeomorphic to $\widetilde{G} / \widetilde{\Gamma}$, where $\widetilde{G}:=\mathbb{R} \ltimes_{\widetilde{\mu}} U_{3}(\mathbb{R})$,

$$
\widetilde{\mu}(t)((x, y, z))=\left(\cos (t \pi) x-\sin (t \pi) y, \sin (t \pi) x+\cos (t \pi) y, z+h_{t}(x, y)\right),
$$


$h_{t}(x, y)=\frac{1}{2} \sin (t \pi)\left(\cos (t \pi)\left(x^{2}-y^{2}\right)-2 \sin (t \pi) x y\right)$ and $\widetilde{\Gamma}=\mathbb{Z} \ltimes_{\widetilde{\mu}}\left\langle\gamma_{1}, \gamma_{2}, \gamma_{3}\right\rangle$ such that $\left[\gamma_{1}, \gamma_{2}\right]=\gamma_{3}^{n}, \widetilde{\mu}(1)\left(\gamma_{1}\right)=\gamma_{1}^{-1} \gamma_{3}^{c_{1}}$ and $\widetilde{\mu}(1)\left(\gamma_{2}\right)=\gamma_{2}^{-1} \gamma_{3}^{c_{2}}$. (Using the addition theorems for sin and cos, one calculates that $\widetilde{\mu}$ is a one-parameter group in $\mathrm{A}\left(U_{3}(\mathbb{R})\right)$.) By [30, Section 3.5], $\widetilde{G} / \widetilde{\Gamma}$ is a secondary Kodaira surface.

Obviously, the Lie algebra of $\widetilde{G}$ is not completely solvable and we cannot use its Chevalley-Eilenberg complex for computing $b_{1}(G / \Gamma)$. But since

$$
\Gamma=\left\langle e_{0}, \ldots, e_{3} \mid e_{0} e_{1} e_{0}^{-1}=e_{1}^{-1} e_{3}^{c_{1}}, e_{0} e_{2} e_{0}^{-1}=e_{2}^{-1} e_{3}^{c_{1}},\left[e_{0}, e_{3}\right]=1,\left[e_{1}, e_{2}\right]=e_{3}^{n}\right\rangle,
$$

we see $b_{1}(G / \Gamma)=\operatorname{rank} \Gamma_{a b}=1$ and $G / \Gamma$ belongs to the last row in Table 2 ,

Case B.3.: $\left.\widetilde{\lambda}_{1}={\widetilde{\lambda_{2}}}=\exp (i \varphi) \in \mathbb{C} \backslash \mathbb{R}, \varphi \in\right] 0,2 \pi[\backslash\{\pi\}$

This case is similar to the last one. We have $|\operatorname{tr} \bar{\nu}(1)| \leq\left|\widetilde{\lambda}_{1}\right|+\left|\widetilde{\lambda}_{2}\right|=2$ and $[23$, Lemma 1] implies that we can assume $\nu(1)$ to be $\left(\begin{array}{cc}0 & -1 \\ 1 & 0\end{array}\right)$ or $\pm\left(\begin{array}{cc}0 & -1 \\ 1 & 1\end{array}\right)$. In each case, one computes $b_{1}(G / \Gamma)=\operatorname{rank} \Gamma_{a b}=1$, as above. Moreover, one embeds a lattice $\widetilde{\Gamma}$ isomorphic to $\Gamma$ in the Lie group $\widetilde{G}:=\mathbb{R} \ltimes_{\widetilde{\mu}} U_{3}(\mathbb{R})$, where

$$
\widetilde{\mu}(t)((x, y, z))=\left(\cos (t \varphi) x-\sin (t \varphi) y, \sin (t \varphi) x+\cos (t \varphi) y, z+h_{t}(x, y)\right),
$$

$h_{t}(x, y)=\frac{1}{2} \sin (t \varphi)\left(\cos (t \varphi)\left(x^{2}-y^{2}\right)-2 \sin (t \varphi) x y\right)$. Again, $\widetilde{G} / \widetilde{\Gamma}$ is a secondary Kodaira surface and $G / \Gamma$ is an example for case i). For more details see [30, Section 3.5].

Below, we give examples for each of the nine types of four-dimensional solvmanifolds. The Lie algebras of the connected and simply-connected four-dimensional solvable Lie groups that admit lattices are listed in Table 8 in Appendix A.

Example. The following manifolds belong to the corresponding row in Table 2 ,

a) $\mathbb{R}^{4} / \mathbb{Z}^{4}$

b) $\left(\mathbb{R} \ltimes_{\mu_{b}} \mathbb{R}^{3}\right) /\left(\mathbb{Z} \ltimes_{\mu_{b}} \mathbb{Z}^{3}\right), \mu_{b}(t)=\left(\begin{array}{lll}1 & 0 & 0 \\ 0 & 1 & t \\ 0 & 0 & 1\end{array}\right)$

c) $\left(\mathbb{R} \ltimes_{\mu_{c}} \mathbb{R}^{3}\right) / \Gamma_{c}$ with

$$
\Gamma_{c}=\mathbb{Z} \ltimes_{\mu_{c}}\left\langle\left(\begin{array}{l}
1 \\
0 \\
0
\end{array}\right),\left(\begin{array}{l}
0 \\
1 \\
1
\end{array}\right),\left(\begin{array}{c}
0 \\
\frac{18+8 \sqrt{5}}{7+3 \sqrt{5}} \\
\frac{2}{3+\sqrt{5}}
\end{array}\right)\right\rangle_{\mathbb{Z}},
$$

$t_{1}=\ln \left(\frac{3+\sqrt{5}}{2}\right)$ and $\mu_{c}(t)=\left(\begin{array}{ccc}1 & 0 & 0 \\ 0 & e^{t t_{1}} & 0 \\ 0 & 0 & e^{-t t_{1}}\end{array}\right)$; the proof that this is really a solvmanifold is analogous to that in the example on page 25. 
d) $\left(\mathbb{R} \ltimes_{\mu_{d}} \mathbb{R}^{3}\right) /\left(\pi \mathbb{Z} \ltimes_{\mu_{d}} \mathbb{Z}^{3}\right), \mu_{d}(t)=\left(\begin{array}{ccc}1 & 0 & 0 \\ 0 & \cos (t) & -\sin (t) \\ 0 & \sin (t) & \cos (t)\end{array}\right)$

e) $\left(\mathbb{R} \ltimes_{\mu_{e}} \mathbb{R}^{3}\right) /\left(\mathbb{Z} \ltimes_{\mu_{e}} \mathbb{Z}^{3}\right), \mu_{e}(t)=\left(\begin{array}{ccc}1 & t & \frac{1}{2}\left(t^{2}-t\right) \\ 0 & 1 & t \\ 0 & 0 & 1\end{array}\right)$

f) Consider $A:=\left(\begin{array}{ccc}0 & 0 & 1 \\ 1 & 0 & -11 \\ 0 & 1 & 8\end{array}\right) \in \mathrm{SL}(3, \mathbb{Z})$. A has $X^{3}-8 X^{2}+11 X-1$ as characteristic polynomial which possesses three pairwise different real roots $t_{1} \approx 6,271, t_{2} \approx 1,631$ and $t_{3} \approx 0,098$. Therefore, $A$ is conjugate to $\mu_{f}(1)$, where $\mu_{f}(t)=\left(\begin{array}{ccc}e^{t} \ln \left(t_{1}\right) & 0 & 0 \\ 0 & e^{t \ln \left(t_{2}\right)} & 0 \\ 0 & 0 & e^{t \ln \left(t_{3}\right)}\end{array}\right)$, and this implies the existence of a lattice $\Gamma_{f}$ in the completely solvable Lie group $\mathbb{R} \ltimes_{\mu_{f}} \mathbb{R}^{3}$.

g) Let $A:=\left(\begin{array}{ccc}0 & 0 & 1 \\ 1 & 0 & -8 \\ 0 & 1 & 4\end{array}\right) \in \operatorname{SL}(3, \mathbb{Z})$. The characteristic polynomial of $A$ is $X^{3}-4 X^{2}+8 X-1$ which has three pairwise different roots $t_{1} \approx 0,134$ and $t_{2,3}=\left(1 / \sqrt{t_{1}}\right)(\cos (\varphi) \pm i \sin (\varphi)) \approx 1,933 \pm 1,935 i$. So $A$ is conjugate to $\mu_{g}(1)$, where $\mu_{g}(t)=\left(\begin{array}{ccc}e^{t \ln \left(t_{1}\right)} & 0 & 0 \\ 0 & e^{t \ln \left(\left|t_{2}\right|\right)} \cos (t \varphi) & -e^{t \ln \left(\left|t_{2}\right|\right)} \sin (t \varphi) \\ 0 & e^{t \ln \left(\left|t_{2}\right|\right)} \sin (t \varphi) & e^{t \ln \left(\left|t_{2}\right|\right)} \cos (t \varphi)\end{array}\right)$, and this implies the existence of a lattice $\Gamma_{g}$ in the Lie group $\mathbb{R} \ltimes_{\mu_{g}} \mathbb{R}^{3}$.

h) Using Theorem 2.1, one shows that

$$
\begin{aligned}
\gamma_{1} & :=\left(1,1,-\frac{1+\sqrt{5}}{3+\sqrt{5}}\right) \\
\gamma_{2} & :=\left(-\frac{2(2+\sqrt{5})}{3+\sqrt{5}}, \frac{1+\sqrt{5}}{3+\sqrt{5}},-\frac{11+5 \sqrt{5}}{7+3 \sqrt{5}}\right), \\
\gamma_{3} & :=(0,0, \sqrt{5})
\end{aligned}
$$

generate a lattice $\Gamma$ in $U_{3}(\mathbb{R})$ with $\left[\gamma_{1}, \gamma_{2}\right]=\gamma_{3}$ and $\gamma_{3}$ central.

Define the one-parameter group $\mu_{h}: \mathbb{R} \rightarrow \mathrm{A}\left(U_{3}(\mathbb{R})\right)$ by

$$
\mu_{h}(t)((x, y, z))=\left(e^{-t t_{1}} x, e^{t t_{1}} y, z\right),
$$

where $t_{1}:=\ln \left(\frac{3+\sqrt{5}}{2}\right)$. Then $\mu_{h}(1)$ preserves the lattice $\Gamma$ with

$$
\mu_{h}(1)\left(\gamma_{1}\right)=\gamma_{1}^{2} \gamma_{2}, \mu_{h}(1)\left(\gamma_{2}\right)=\gamma_{1} \gamma_{2}, \mu_{h}(1)\left(\gamma_{3}\right)=\gamma_{3}
$$

and therefore, $\mathbb{Z} \ltimes_{\mu_{h}} \Gamma$ is a lattice in $\mathbb{R} \ltimes_{\mu_{h}} U_{3}(\mathbb{R})$. 
i) Consider the Lie group $\widetilde{G}$ and the one-parameter group $\widetilde{\mu}$ of Case B.2 from the proof of the last theorem. Setting $\gamma_{1}=(1,0,0), \gamma_{2}=(0,1,0)$ as well as $\gamma_{3}=(0,0,1), n=1$ and $c_{1}=c_{2}=0$, one explicitly gets an example.

The manifolds of type c) show that formal spaces with the same minimal model as a Kähler manifold need not be Kählerian. This was proved by Fernández and Gray.

Theorem 6.3 ([19]). Let $M$ be one of the symplectic solvmanifolds of type $c)$ in the last theorem, i.e. $M$ is formal and possesses no complex structure. $M$ has the same minimal model as the Kähler manifold $T^{2} \times S^{2}$.

\section{$7 \quad$ Five-dimensional solvmanifolds}

We study the five-dimensional solvmanifolds by regarding lattices in the corresponding connected and simply-connected Lie groups. By Proposition 3.3, their Lie algebras have to be unimodular. These are listed in Appendix A.

\subsection{Nilpotent and decomposable solvable Lie algebras}

There are nine classes of nilpotent Lie algebras in dimension five, see Table 9. Each of them has a basis with rational structure constants. By Theorem 2.1, the corresponding connected and simply-connected Lie groups admit lattices and accordingly to Theorem 2.7, the associated nilmanifolds are formal if and only if they are tori. For $i \in\{4,5,6\}$ the connected and simply-connected nilpotent Lie group with Lie algebra $\mathfrak{g}_{5 . i}$ possesses the left-invariant contact form $x_{1}$ (where $x_{1}$ is dual to the basis element $X_{1} \in \mathfrak{g}_{\mathfrak{i}}$ as in Table 9). Therefore, the corresponding nilmanifolds are contact.

The eight classes of decomposable unimodular non-nilpotent solvable Lie algebras are listed in Table 10. Except for $\mathfrak{g}_{4.2} \oplus \mathfrak{g}_{1}$, the corresponding connected and simply-connected Lie groups admit lattices since both of their factors admit lattices.

Theorem 7.1.1. The connected and simply-connected Lie group $G_{4.2} \times \mathbb{R}$ with Lie algebra $\mathfrak{g}_{4.2} \oplus \mathfrak{g}_{1}$ possesses no lattice.

Proof. Write $G$ for $G_{4.2} \times \mathbb{R}$ and

$$
\mathfrak{g}=\left\langle X_{1}, \ldots, X_{5} \mid\left[X_{1}, X_{4}\right]=-2 X_{1},\left[X_{2}, X_{4}\right]=X_{2},\left[X_{3}, X_{4}\right]=X_{2}+X_{3}\right\rangle
$$

for its Lie algebra which has $\mathfrak{n}=\mathbb{R}_{X_{1}, X_{2}, X_{3}, X_{5}}^{4}$ as nilradical. Therefore, $G$ can be written as almost abelian Lie group $\mathbb{R} \ltimes_{\mu} \mathbb{R}^{4}$ with

$$
\mu(t)=\exp ^{G L(n, \mathbb{R})}\left(t \operatorname{ad}\left(X_{4}\right)\right)=\left(\begin{array}{cccc}
e^{2 t} & 0 & 0 & 0 \\
0 & e^{-t} & -t e^{-t} & 0 \\
0 & 0 & e^{-t} & 0 \\
0 & 0 & 0 & 1
\end{array}\right)
$$


By Corollary 4.5, the existence of a lattice in $G$ would imply that there is $t_{1} \in$ $\mathbb{R} \backslash\{0\}$ such that $\mu\left(t_{1}\right)$ is conjugate to an element of $\operatorname{SL}(4, \mathbb{Z})$. Clearly, the characteristic polynomial of $\mu\left(t_{1}\right)$ is $P(X)=(X-1) \widetilde{P}(X)$, where the polynomial $\widetilde{P}(X)=X^{3}-k X^{2}+l X-1 \in \mathbb{Z}[X]$ has the double root $e^{-t_{1}}$. Lemma B.4 then implies $e^{-t_{1}}=1$, i.e. $t_{1}=0$ which is a contradiction.

Proposition 7.1.2. If $\Gamma$ is a lattice in a five-dimensional completely solvable non-nilpotent connected and simply-connected decomposable Lie group $G$, then $G / \Gamma$ is formal.

Proof. Let $G, \Gamma$ be as in the proposition. As usual, we denote by $\mathfrak{g}$ the Lie algebra of $G$. We have $\mathfrak{g}=\mathfrak{h} \oplus k \mathfrak{g}_{1}$ with $k \in\{1,2\}$ and a certain $(5-k)$ dimensional completely solvable non-nilpotent Lie algebra $\mathfrak{h}$, see Tables 10 and 8. By completely solvability and Theorem 3.11 (ii), $G / \Gamma$ and the ChevalleyEilenberg complex of $\mathfrak{h} \oplus k \mathfrak{g}_{1}$ share their minimal model $\mathcal{M}$. The lower dimensional discussion above shows that for all $\mathfrak{h}$ which can arise in the decomposition of $\mathfrak{g}$ the algebras $\mathcal{M}_{\left(\bigwedge \mathfrak{h}^{*}, \delta_{\mathfrak{h}}\right)}$ and $\mathcal{M}_{\left(\bigwedge k \mathfrak{g}_{1}^{*}, \delta=0\right)}=\left(\bigwedge k \mathfrak{g}_{1}^{*}, \delta=0\right)$ are formal. This implies the formality of $\mathcal{M}=\mathcal{M}_{\left(\bigwedge \mathfrak{h}^{*}, \delta_{\mathfrak{h}}\right)} \otimes \mathcal{M}_{\left(\bigwedge k \mathfrak{g}_{1}^{*}, \delta=0\right)}$.

\subsection{Indecomposable non-nilpotent Lie algebras}

There are 19 classes of indecomposable non-nilpotent Lie algebras in dimension five which are unimodular. These are listed in Tables 11-14. Instead of the small German letters for the Lie algebras in the mentioned tables, we use capital Latin letters (with the same subscripts) for the corresponding connected and simply-connected Lie groups.

We want to examine which of them admit lattices and where appropriate, whether the quotients are formal. The non-existence proofs of lattices in certain almost abelian Lie groups below are taken from Harshavardhan's thesis [28]. Some of the existence proofs of lattices in certain almost abelian Lie groups are sketched in [28, pp. 29 and 30].

\section{Almost abelian algebras}

We now consider the almost abelian Lie groups $G_{5 . i}=\mathbb{R} \ltimes_{\mu_{i}} \mathbb{R}^{4}$. We write $\mu(t)=\mu_{i}(t)=\exp ^{G L(4, \mathbb{R})}\left(t \operatorname{ad}\left(X_{5}\right)\right)$, where $X_{5} \in \mathfrak{g}_{5 . i}$ is as in Table 11 $\left(X_{5}\right.$ depends on $i$ ). We know by Corollary 4.5, Theorem 4.6 and Proposition 4.7 that there is a lattice $\Gamma$ in $G_{5 . i}$ if and only if there exists $t_{1} \neq 0$ such that $\mu\left(t_{1}\right)$ is conjugate to $\widetilde{\mu}(1) \in \mathrm{SL}(4, \mathbb{Z})$ and $\Gamma=\mathbb{Z} \ltimes_{\widetilde{\mu}} \mathbb{Z}^{4}$. This will be used in the proof of the following propositions.

Methods to obtain integer matrices with given characteristic polynomial and necessary conditions for their existence are given in Appendix B. 
Proposition 7.2.1. Let $p, q, r \in \mathbb{R}$ with $-1 \leq r \leq q \leq p \leq 1$, pqr $\neq 0$ and $p+q+r=-1$. If the completely solvable Lie group $G_{5.7}^{p, q, r}$ admits a lattice and $M$ denotes the corresponding solvmanifold, then $M$ is formal, $b_{1}(M)=1$ and one of the following conditions holds:

(i) $b_{2}(M)=0$,

(ii) $b_{2}(M)=2$, i.e. $\left.r=-1, p=-q \in\right] 0,1[$ or

(iii) $b_{2}(M)=4$, i.e. $r=q=-1, p=1$.

Moreover, there exist $p, q, r$ as above satisfying (i), (ii) resp. (iii) such that $G_{5.7}^{p, q, r}$ admits a lattice.

Proof. We suppress the sub- and superscripts of $G$ and $\mathfrak{g}$.

a) Assume, there is a lattice in $G$ and denote the corresponding solvmanifold by $M$. Since $\mathfrak{g}$ is completely solvable, the inclusion of the Chevallier-Eilenberg complex $\left(\bigwedge\left(x_{1}, \ldots, x_{5}\right), \delta\right)$ into the forms on $M$ induces an isomorphism on cohomology. Moreover, the minimal model of $\left(\bigwedge\left(x_{1}, \ldots, x_{5}\right), \delta\right)$ is isomorphic to the minimal model of $M$.

$\delta$ is given by

$$
\delta x_{1}=-x_{15}, \delta x_{2}=-p x_{25}, \delta x_{3}=-q x_{35}, \delta x_{4}=-r x_{45}, \delta x_{5}=0 .
$$

(Here we write $x_{i j}$ for $x_{i} x_{j}$.) This implies $b_{1}(M)=1$.

One computes the differential of the non-exact generators of degree two in the Chevalley-Eilenberg complex as

$$
\begin{array}{lll}
\delta x_{12}=(1+p) x_{125}, & \delta x_{13}=(1+q) x_{135}, & \delta x_{14}=(1+r) x_{145} \\
\delta x_{23}=(p+q) x_{235}, & \delta x_{24}=(p+r) x_{245}, & \delta x_{34}=(q+r) x_{345}
\end{array}
$$

$-1 \leq r \leq q \leq p \leq 1, p q r \neq 0$ and $p+q+r=-1$ implies $p \neq-1$ and $q \neq-r$ and a short computation yields that either (i), (ii) or (iii) holds.

In each case, we determine the 2-minimal model, i.e. the minimal model up to generators of degree two and will see, that these generators are closed. By Definition 1.1.4, the minimal model then is 2-formal and Theorem 1.1.6 implies the formality of $M$.

If we are in case (i), the minimal model has one closed generator of degree one, and no generator of degree two.

If we are in case (ii), we have $r=-1, p=-q \in] 0,1[$,

$$
\begin{array}{ll}
\delta x_{12}=(1+p) x_{125} \neq 0, & \delta x_{13}=(1-p) x_{135} \neq 0, \quad \delta x_{14}=0, \\
\delta x_{23}=0, & \delta x_{24}=(p-1) x_{245} \neq 0, \quad \delta x_{34}=(-1-p) x_{345} \neq 0, \\
& H^{1}(M) \cong \quad\left\langle\left[x_{5}\right]\right\rangle, \\
& H^{2}(M) \cong\left\langle\left[x_{14}\right],\left[x_{23}\right]\right\rangle,
\end{array}
$$


and the 2-minimal model $\rho:\left(\bigwedge V^{\leq 2}, d\right) \rightarrow\left(\bigwedge\left(x_{1}, \ldots, x_{5}\right), \delta\right)$ is given by

$$
\begin{aligned}
& \rho(y)=x_{5}, \quad|y|=1, \quad d y=0 \\
& \rho\left(z_{1}\right)=x_{14}, \quad\left|z_{1}\right|=2, \quad d z_{1}=0 \text {; } \\
& \rho\left(z_{2}\right)=x_{23}, \quad\left|z_{2}\right|=2, \quad d z_{2}=0 .
\end{aligned}
$$

Note, further generators of degree $\leq 2$ do not occur, since $y^{2}=0$ (by graded commutativity) and $\rho\left(y z_{i}\right)$ is closed and non-exact. Here we use the construction of the minimal model that we have given in the proof of Theorem 1.1.2.

Case (iii) is similar to case (ii).

b) Now, we show that there are examples for each of the three cases. In case (i), we follow [28] and consider the matrix $\left(\begin{array}{cccc}1 & 0 & 0 & -2 \\ 1 & 2 & 0 & -3 \\ 0 & 1 & 3 & 5 \\ 0 & 0 & 1 & 2\end{array}\right)$. It suffices to show that there are $t_{1} \in \mathbb{R} \backslash\{0\},-1<r<q<p<1$ with $p q r \neq 0, p \neq-q, p \neq-r$, $q \neq-r$ and $p+q+r=-1$ such that

$$
\mu\left(t_{1}\right)=\exp ^{G L(4, \mathbb{R})}\left(t_{1} \operatorname{ad}\left(X_{5}\right)\right)=\left(\begin{array}{cccc}
e^{-t_{1}} & 0 & 0 & 0 \\
0 & e^{-p t_{1}} & 0 & 0 \\
0 & 0 & e^{-q t_{1}} & 0 \\
0 & 0 & 0 & e^{-r t_{1}}
\end{array}\right)
$$

is conjugate to the matrix above, which has $P(X)=X^{4}-8 X^{3}+18 X^{2}-10 X+1$ as characteristic polynomial. $P$ has four distinct roots $\lambda_{1}, \ldots, \lambda_{4}$ with $\lambda_{1} \approx 0,12$, $\lambda_{2} \approx 0,62, \lambda_{3} \approx 2,79$ and $\lambda_{4} \approx 4,44$. Define $t_{1}:=-\ln \left(\lambda_{1}\right)$ and $p, q, r$ by $e^{-p t_{1}}=\lambda_{2}, e^{-q t_{1}}=\lambda_{3}$ and $e^{-r t_{1}}=\lambda_{4}$. Then $t_{1}, p, q, r$ have the desired properties.

In case (ii), regard the matrix $\left(\begin{array}{cccc}0 & 0 & 0 & -1 \\ 1 & 0 & 0 & 10 \\ 0 & 1 & 0 & -23 \\ 0 & 0 & 1 & 10\end{array}\right)$ which is conjugate to $\mu\left(t_{1}\right)=\left(\begin{array}{cccc}e^{-t_{1}} & 0 & 0 & 0 \\ 0 & e^{-p t_{1}} & 0 & 0 \\ 0 & 0 & e^{p t_{1}} & 0 \\ 0 & 0 & 0 & e^{t_{1}}\end{array}\right)$ for $t_{1}=2 \ln \left(\frac{3+\sqrt{5}}{2}\right)$ and $p=\frac{1}{2}$ since both matrices have the same characteristic polynomial which has four distinct real roots.

In case (iii), regard the matrix $\left(\begin{array}{cccc}3 & 0 & -1 & 0 \\ 0 & 3 & 0 & -1 \\ 1 & 0 & 0 & 0 \\ 0 & 1 & 0 & 0\end{array}\right)$ which is conjugate to $\mu\left(t_{1}\right)=\left(\begin{array}{cccc}e^{-t_{1}} & 0 & 0 & 0 \\ 0 & e^{-t_{1}} & 0 & 0 \\ 0 & 0 & e^{t_{1}} & 0 \\ 0 & 0 & 0 & e^{t_{1}}\end{array}\right)$ for $t_{1}=\ln \left(\frac{3+\sqrt{5}}{2}\right)$ since both matrices have the 
same minimal polynomial by Proposition B.8 (ii).

We have seen that a non-formal solvmanifold is a non-toral nilmanifold in dimensions three and four. In higher dimensions this is no longer true as the following proposition shows:

Proposition 7.2.2. The completely solvable Lie group $G_{5.8}^{-1}$ admits a lattice.

Moreover, for each lattice $\Gamma$ the corresponding solvmanifold $M=G_{5.8}^{-1} / \Gamma$ has $b_{1}(M)=2$ and is not formal.

Proof. Again, we suppress the sub- and superscripts. $G$ admits a lattice since $\mu(t)=\exp ^{G L(4, \mathbb{R})}\left(t \operatorname{ad}\left(X_{5}\right)\right)=\left(\begin{array}{cccc}1 & -t & 0 & 0 \\ 0 & 1 & 0 & 0 \\ 0 & 0 & e^{-t} & 0 \\ 0 & 0 & 0 & e^{t}\end{array}\right)$ and $\left(\begin{array}{cccc}0 & 0 & 0 & -1 \\ 1 & 0 & 0 & 5 \\ 0 & 1 & 0 & -8 \\ 0 & 0 & 1 & 5\end{array}\right)$ are conjugated for $t_{1}=\ln \left(\frac{3+\sqrt{5}}{2}\right)$. Note that the transformation matrix $T \in \operatorname{GL}(4, \mathbb{R})$ with $T A T^{-1}=\mu\left(t_{1}\right)$ is

$$
T=\left(\begin{array}{cccc}
1 & 0 & -1 & -2 \\
\frac{1}{\ln \left(\frac{3+\sqrt{5}}{2}\right)} & \frac{1}{\ln \left(\frac{3+\sqrt{5}}{2}\right)} & \frac{1}{\ln \left(\frac{3+\sqrt{5}}{2}\right)} & \frac{1}{\ln \left(\frac{3+\sqrt{5}}{2}\right)} \\
-\frac{5+3 \sqrt{5}}{10} & -\frac{1}{\sqrt{5}} & \frac{5-3 \sqrt{5}}{10} & \frac{3}{2}-\frac{7}{2 \sqrt{5}} \\
\frac{-5+3 \sqrt{5}}{10} & \frac{1}{\sqrt{5}} & \frac{5+3 \sqrt{5}}{10} & \frac{3}{2}+\frac{7}{2 \sqrt{5}}
\end{array}\right) .
$$

Now, let $\Gamma$ be an arbitrary lattice in $G$. By completely solvability and Theorem 3.11 (ii), we get the minimal model of $M=G / \Gamma$ as the minimal model $\mathcal{M}$ of the Chevalley-Eilenberg complex $\left(\bigwedge \mathfrak{g}^{*}, \delta\right)$. The latter is given by

$$
\delta x_{1}=-x_{25}, \delta x_{2}=0, \delta x_{3}=-x_{35}, \delta x_{4}=x_{45}, \delta x_{5}=0,
$$

which implies $b_{1}(M)=2$. Further, the minimal model $\rho:(\bigwedge V, d) \rightarrow\left(\bigwedge \mathfrak{g}^{*}, \delta\right)$ must contain two closed generators $y_{1}, y_{2}$ which map to $x_{2}$ and $x_{5}$. Then we have $\rho\left(y_{1} y_{2}\right)=x_{25}=-\delta x_{1}$ and the minimal model's construction in the proof of Theorem 1.1.2 implies that there is another generator $u$ of degree one such that $\rho(u)=-x_{1}$ and $d u=y_{1} y_{2}$. Since $\rho\left(u y_{1}\right)=-x_{12}$ and $\rho\left(u y_{2}\right)=-x_{15}$ are closed and non-exact, there are no further generators of degree one in $V$. But this implies that $(u+c) y_{1}$ is closed and non-exact in $\mathcal{M}$ for each closed element $c$ of degree one. Using the notation of Theorem 1.1.5, we have $u \in N^{1}, y_{1} \in V^{1}$ and $\mathcal{M}$ is not formal.

Proposition 7.2.3. The completely solvable Lie group $G_{5.9}^{p,-2-p}, p \geq-1$, does not admit a lattice.

Proof. The first half of the proof is taken from [28]. Assume there is a lattice. $\mu(t)=\left(\begin{array}{cccc}e^{-t} & -t e^{-t} & 0 & 0 \\ 0 & e^{-t} & 0 & 0 \\ 0 & 0 & e^{-t p} & 0 \\ 0 & 0 & 0 & e^{t(2+p)}\end{array}\right)$ is conjugate to an element of $\operatorname{SL}(4, \mathbb{Z})$ for 
$t=t_{1} \neq 0$ and has roots $e^{-t_{1}}, e^{-t_{1}}, e^{-t_{1} p}$ and $e^{t_{1}(2+p)}$. By Proposition B.6. this can occur if and only if $p=-1$. Therefore, for the remainder of the proof we assume $p=-1$.

The Jordan form of $\mu\left(t_{1}\right)$ is $\left(\begin{array}{cccc}e^{-t} & 1 & 0 & 0 \\ 0 & e^{-t} & 0 & 0 \\ 0 & 0 & e^{t} & 0 \\ 0 & 0 & 0 & e^{t}\end{array}\right)$, i.e. the characteristic and the minimal polynomial of $\mu\left(t_{1}\right)$ are

$$
\begin{aligned}
P(X) & =\left(X-e^{-t_{1}}\right)^{2}\left(X-e^{t_{1}}\right)^{2} \\
& =X^{4}-2\left(e^{-t_{1}}+e^{t_{1}}\right) X^{3}+\left(e^{-2 t_{1}}+e^{2 t_{1}}+4\right) X^{2}-2\left(e^{-t_{1}}+e^{t_{1}}\right) X+1, \\
m(X) & =\left(X-e^{-t_{1}}\right)^{2}\left(X-e^{t_{1}}\right) \\
& =X^{3}-\left(2 e^{-t_{1}}+e^{t_{1}}\right) X^{2}+\left(e^{-2 t_{1}}+2\right) X-e^{-t_{1}} .
\end{aligned}
$$

Since $\mu\left(t_{1}\right)$ is conjugate to an integer matrix, we have $P(X), m(X) \in \mathbb{Z}[X]$ by Theorem B.3. This is impossible for $t_{1} \neq 0$.

Proposition 7.2.4 ([28]). The completely solvable Lie group $G_{5.11}^{-3}$ does not admit a lattice.

Proof. If the group admits a lattice, there exists $t_{1} \in \mathbb{R} \backslash\{0\}$ such that the characteristic polynomial of $\mu\left(t_{1}\right)=\left(\begin{array}{cccc}e^{-t_{1}} & -t_{1} e^{-t_{1}} & \frac{t_{1}^{2}}{2} e^{-t_{1}} & 0 \\ 0 & e^{-t_{1}} & -t_{1} e^{-t_{1}} & 0 \\ 0 & 0 & e^{-t_{1}} & 0 \\ 0 & 0 & 0 & e^{3 t_{1}}\end{array}\right)$ is a monic integer polynomial with a three-fold root $e^{-t_{1}}$ and a simple root $e^{3 t_{1}}$. By Proposition B.6, this is impossible for $t_{1} \neq 0$.

Proposition 7.2.5. There are $q, r \in \mathbb{R}$ with $-1 \leq q<0, q \neq-\frac{1}{2}, r \neq 0$ such that $G_{5.13}^{-1-2 q, q, r}$ admits a lattice.

Proof. We have $\mu_{q, r}(t)=\left(\begin{array}{cccc}e^{-t} & 0 & 0 & 0 \\ 0 & e^{t+2 q t} & 0 & 0 \\ 0 & 0 & e^{-q t} \cos (r t) & -e^{-q t} \sin (r t) \\ 0 & 0 & e^{-q t} \sin (r t) & e^{-q t} \cos (r t)\end{array}\right)$ and as claimed in [28], there exist $t_{1} \neq 0, q_{0}, r_{0}$ such that $\mu_{q_{0}, r_{0}}\left(t_{1}\right)$ is conjugate to $A:=\left(\begin{array}{llll}1 & 0 & 0 & 1 \\ 1 & 2 & 0 & 2 \\ 0 & 1 & 3 & 0 \\ 0 & 0 & 1 & 0\end{array}\right)$ which implies the existence of a lattice $\Gamma_{A}$ in $G_{5.13}^{-1-2 q_{0}, q_{0}, r_{0}}$.

If $\lambda_{1} \approx 0,15<\lambda_{2} \approx 3,47$ denote the real roots and $\lambda_{3,4} \approx 1,17 \pm i 0,67$ the non-real roots of $P_{A}(X)=X^{4}-6 X^{3}+11 X^{2}-8 X+1$, then $t_{1}=-\ln \left(\lambda_{1}\right) \approx 1,86$, $q_{0}=\frac{1}{2}\left(\frac{\ln \left(\lambda_{2}\right)}{t_{1}}-1\right) \approx-0,16$ and $r_{0}=\frac{1}{t_{1}} \arccos \left(\operatorname{Re}\left(\lambda_{3}\right) e^{q_{0} t_{1}}\right) \approx 0,27$. 
Remark. If the real number $\frac{\pi}{t_{1} r_{0}}$ is not rational, then Theorems 3.11 (iii) and 4.8 enable us to show that the manifold $G_{5.13}^{-1-2 q_{0}, q_{0}, r_{0}} / \Gamma_{A}$ has $b_{1}=1$ and is formal.

Proposition 7.2.6. There exists $r \in \mathbb{R} \backslash\{0\}$ such that $G_{5.13}^{-1,0, r}$ admits a lattice. Proof. Let $t_{1}=\ln \left(\frac{3+\sqrt{5}}{2}\right), r=\pi / t_{1}$ and $A=\left(\begin{array}{cccc}3 & 1 & 0 & 0 \\ -1 & 0 & 0 & 0 \\ 0 & 0 & -1 & 0 \\ 0 & 0 & 0 & -1\end{array}\right)$. Then $A$ is conjugate to $\mu_{0, r}\left(t_{1}\right)=\left(\begin{array}{cccc}e^{-t_{1}} & 0 & 0 & 0 \\ 0 & e^{t_{1}} & 0 & 0 \\ 0 & 0 & \cos \left(r t_{1}\right) & \sin \left(r t_{1}\right) \\ 0 & 0 & \sin \left(r t_{1}\right) & \cos \left(r t_{1}\right)\end{array}\right)$ and this implies the existence of a lattice.

$$
\text { Note that we have } T A T^{-1}=\mu_{0, r}\left(t_{1}\right) \text {, where } T=\left(\begin{array}{cccc}
1 & \frac{18+8 \sqrt{5}}{7+3 \sqrt{5}} & 0 & 0 \\
1 & \frac{2}{3+\sqrt{5}} & 0 & 0 \\
0 & 0 & 1 & 0 \\
0 & 0 & 0 & 1
\end{array}\right) \text {. }
$$

Remark. Since the abelianisation of the lattice in the last proof is isomorphic to $\mathbb{Z} \oplus \mathbb{Z}_{2}^{2}$, the constructed solvmanifold has $b_{1}=1$.

Proposition 7.2.7. $G_{5.14}^{0}$ admits a lattice.

Proof. We have $\mu(t)=\left(\begin{array}{cccc}1 & -t & 0 & 0 \\ 0 & 1 & 0 & 0 \\ 0 & 0 & \cos (t) & -\sin (t) \\ 0 & 0 & \sin (t) & \cos (t)\end{array}\right)$. Let $t_{1}=\frac{\pi}{3}$, then $\mu\left(t_{1}\right)$ is conjugate to $\left(\begin{array}{cccc}1 & 0 & 0 & 0 \\ 1 & 1 & 0 & 0 \\ 0 & 1 & 1 & -1 \\ 0 & 0 & 1 & 0\end{array}\right)$, so there is a lattice. Note that the matrix $T=\left(\begin{array}{cccc}-1 & 1 & 0 & 0 \\ -\frac{\pi}{3} & 0 & 0 & 0 \\ -\frac{1}{\sqrt{3}} & -\frac{1}{\sqrt{3}} & \frac{2}{\sqrt{3}} & -\frac{1}{\sqrt{3}} \\ 1 & -1 & 0 & 1\end{array}\right) \in \mathrm{GL}(4, \mathbb{R})$ satisfies $T A T^{-1}=\mu\left(t_{1}\right)$.

Remark. The abelianisation of the lattice in the last proof is isomorphic to $\mathbb{Z}^{2}$, i.e. the corresponding solvmanifold has $b_{1}=2$.

Proposition 7.2.8. If there is a lattice $\Gamma$ in the Lie group $G:=G_{5.14}^{0}$ such that $b_{1}(G / \Gamma)=2$, then $G / \Gamma$ is not formal.

Proof. By Theorem 3.11(i), the natural inclusion of the Chevalley-Eilenberg complex $\left(\bigwedge \mathfrak{g}^{*}, \delta\right) \rightarrow(\Omega(G / \Gamma), d)$ induces an injection on cohomology. $\left(\bigwedge \mathfrak{g}^{*}, \delta\right)$ is given by

$$
\delta x_{1}=-x_{25}, \delta x_{2}=0, \delta x_{3}=-x_{45}, \delta x_{4}=x_{35}, \delta x_{5}=0 .
$$


This implies $b_{1}\left(\bigwedge \mathfrak{g}^{*}, \delta\right)=2$, hence $H^{1}(G / \Gamma, d)=\left\langle\left[x_{2}\right],\left[x_{5}\right]\right\rangle$. Therefore

$$
\left[x_{2}\right] \cdot H^{1}(G / \Gamma)+H^{1}(G / \Gamma) \cdot\left[x_{5}\right]=\left\langle\left[x_{25}\right]\right\rangle=\left\langle\left[\delta x_{1}\right]\right\rangle=0,
$$

and in the Massey product $\left\langle\left[x_{2}\right],\left[x_{2}\right],\left[x_{5}\right]\right\rangle=\left[-x_{15}\right]$ is no indeterminacy. Since $x_{15}$ is closed and not exact, $G / \Gamma$ cannot be formal.

Proposition 7.2.9. The completely solvable Lie group $G_{5.15}^{-1}$ admits a lattice. For each lattice the corresponding solvmanifold satisfies $b_{1}=1$ and is non-formal.

Proof. As we have done above, we suppress the sub- and superscripts. First, we follow [28] and consider the matrix $A:=\left(\begin{array}{cccc}2 & 0 & 0 & -1 \\ 1 & 2 & 0 & 2 \\ 0 & 1 & 1 & 2 \\ 0 & 0 & 1 & 1\end{array}\right)$ which is conjugate to $\mu\left(t_{1}\right)=\left(\begin{array}{cccc}e^{-t_{1}} & -t_{1} e^{-t_{1}} & 0 & 0 \\ 0 & e^{-t_{1}} & 0 & 0 \\ 0 & 0 & e^{t_{1}} & -t_{1} e^{t_{1}} \\ 0 & 0 & 0 & e^{t_{1}}\end{array}\right)$ for $t_{1}=\ln \left(\frac{3+\sqrt{5}}{2}\right)$. This implies the existence of a lattice. The transformation matrix $T \in \mathrm{GL}(4, \mathbb{R})$ with $T A T^{-1}=\mu\left(t_{1}\right)$ is

$$
T=\left(\begin{array}{cccc}
\frac{2}{5 \sqrt{5}} & -\frac{1}{5 \sqrt{5}} & -\frac{2}{5 \sqrt{5}} & \frac{1}{2}+\frac{3}{50} \sqrt{5} \\
-\frac{3+\sqrt{5}}{10 \ln \left(\frac{3+\sqrt{5}}{2}\right)} & \frac{2+\sqrt{5}}{5 \ln \left(\frac{3+\sqrt{5}}{2}\right)} & -\frac{(3+\sqrt{5})^{2}}{20 \ln \left(\frac{3+\sqrt{5}}{2}\right)} & \frac{2+\sqrt{5}}{5 \ln \left(\frac{3+\sqrt{5}}{2}\right)} \\
-\frac{2}{5 \sqrt{5}} & \frac{1}{5 \sqrt{5}} & \frac{2}{5 \sqrt{5}} & \frac{1}{2}-\frac{3}{50} \sqrt{5} \\
-\frac{2}{5(3+\sqrt{5}) \ln \left(\frac{3+\sqrt{5}}{2}\right)} & -\frac{-1+\sqrt{5}}{5(3+\sqrt{5}) \ln \left(\frac{3+\sqrt{5}}{2}\right)} & \frac{-3+\sqrt{5}}{5(3+\sqrt{5}) \ln \left(\frac{3+\sqrt{5}}{2}\right)} & -\frac{-1+\sqrt{5}}{5(3+\sqrt{5}) \ln \left(\frac{3+\sqrt{5}}{2}\right)}
\end{array}\right) .
$$

Let $\Gamma$ be an arbitrary lattice in $G$. By completely solvability and Theorem 3.11 (ii), we get the minimal model of $M=G / \Gamma$ as the minimal model $\mathcal{M}$ of the Chevalley-Eilenberg complex $\left(\bigwedge \mathfrak{g}^{*}, \delta\right)$. The latter is given by

$$
\delta x_{1}=-x_{15}-x_{25}, \delta x_{2}=-x_{25}, \delta x_{3}=x_{35}-x_{45}, \delta x_{4}=x_{45}, \delta x_{5}=0,
$$

which implies $b_{1}(M)=1$.

One computes the differential of the non-exact generators of degree two in the Chevalley-Eilenberg complex as

$$
\begin{array}{ccc}
\delta x_{12}=2 x_{125}, & \delta x_{13}=x_{145}+x_{235}, & \delta x_{14}=x_{245}, \\
\delta x_{23}=x_{245}, & \delta x_{24}=0, & \delta x_{34}=-2 x_{345} .
\end{array}
$$

The minimal model $\rho:(\bigwedge V, d) \rightarrow\left(\bigwedge \mathfrak{g}^{*}, \delta\right)$ must contain three closed generators $y, z_{1}, z_{2}$ which map to $x_{5}, x_{14}-x_{23}$ and $x_{24}$. We see $\rho\left(y z_{1}\right)=x_{145}-x_{235}$ is closed and non-exact, $\rho\left(y z_{2}\right)=x_{245}=\delta x_{23}$ and the minimal model's construction in the proof of Theorem 1.1.2 implies that there is another generator $u$ of degree two 
such that $\rho(u)=x_{23}$ and $d u=y z_{2}$. Since $\rho(u y)=x_{235}$ is closed and non-exact, there are no further generators of degree less than or equal to two in $V$. But this implies that $(u+c) y$ is closed and non-exact in $\mathcal{M}$ for each closed element $c$ of degree two. Using the notation of Theorem 1.1.5, we have $u \in N^{2}, y \in V^{1}$ and $\mathcal{M}$ is not formal.

Proposition $7.2 .10([28]) . G_{5.16}^{-1, q}, q \neq 0$, does not admit a lattice.

Proof. If the group admits a lattice, there exists $t_{1} \in \mathbb{R} \backslash\{0\}$ such that the characteristic polynomial of $\mu\left(t_{1}\right)=\left(\begin{array}{cccc}e^{-t_{1}} & -t_{1} e^{-t_{1}} & 0 & 0 \\ 0 & e^{-t_{1}} & 0 & 0 \\ 0 & 0 & e^{t_{1}} \cos \left(t_{1} q\right) & -e^{t_{1}} \sin \left(t_{1} q\right) \\ 0 & 0 & e^{t_{1}} \sin \left(t_{1} q\right) & e^{t_{1}} \cos \left(t_{1} q\right)\end{array}\right)$ is a monic integer polynomial with simple roots $e^{t_{1}}\left(\cos \left(t_{1} q\right) \pm i \sin \left(t_{1} q\right)\right)$ and a double root $e^{-t_{1}}$. By Proposition B.6, this is impossible for $t_{1} \neq 0$.

Proposition 7.2.11. There are $p, r \in \mathbb{R}, p \neq 0, r \notin\{0, \pm 1\}$, such that $G_{5.17}^{p,-p, r}$ admits a lattice.

Proof. We follow [28].

$$
\begin{aligned}
A & :=\left(\begin{array}{cccc}
2 & 0 & 0 & -11 \\
1 & 2 & 0 & -9 \\
0 & 1 & 1 & -1 \\
0 & 0 & 1 & 1
\end{array}\right) \text { is conjugate to } \\
\mu\left(t_{1}\right) & =\left(\begin{array}{cccc}
e^{-t_{1} p} \cos \left(t_{1}\right) & -e^{-t_{1} p} \sin \left(t_{1}\right) & 0 & 0 \\
e^{-t_{1} p} \sin \left(t_{1}\right) & e^{-t_{1} p} \cos \left(t_{1}\right) & 0 & 0 \\
0 & 0 & e^{t_{1} p} \cos \left(t_{1} r\right) & -e^{t_{1} p} \sin \left(t_{1} r\right) \\
0 & 0 & e^{t_{1} p} \sin \left(t_{1} r\right) & e^{t_{1} p} \cos \left(t_{1} r\right)
\end{array}\right)
\end{aligned}
$$

for certain $t_{1}, p, r \neq 0$, i.e. there is a lattice.

If $\lambda_{1,2} \approx 0,306 \pm i 0,025$ and $\lambda_{3,4} \approx 2,694 \pm i 1,83$ denote the roots of $P_{A}(X)=X^{4}-6 X^{3}+14 X^{2}-7 X+1$, one has $t_{1} p=-\ln \left(\left|\lambda_{1}\right|\right) \approx 1,181$, hence $t_{1}=\arccos \left(\operatorname{Re}\left(\lambda_{1}\right) e^{t_{1} p}\right) \approx 0,062, p \approx 14,361 . t_{1} r=\arccos \left(\operatorname{Re}\left(\lambda_{3}\right) e^{t_{1} p}\right) \approx 0,597$ implies $r \approx 7,259$.

Remark. Since the abelianisation of the lattice in the last proof is isomorphic to $\mathbb{Z} \oplus \mathbb{Z}_{3}$, the corresponding solvmanifold has $b_{1}=1$.

Proposition 7.2.12. There exists $p \in \mathbb{R} \backslash\{0\}$ such that $G_{5.17}^{p,-p, \pm 1}$ admits a lattice.

Proof. Let $p:=\frac{1}{\pi} \ln \left(\frac{3+\sqrt{5}}{2}\right), t_{1}:=\pi$ and $A:=\left(\begin{array}{cccc}0 & -1 & 0 & 0 \\ 1 & -3 & 0 & 0 \\ 0 & 0 & 0 & -1 \\ 0 & 0 & 1 & -3\end{array}\right)$. Then 
$\mu\left(t_{1}\right)=\left(\begin{array}{cccc}e^{-t_{1} p} \cos \left(t_{1}\right) & -e^{-t_{1} p} \sin \left(t_{1}\right) & 0 & 0 \\ e^{-t_{1} p} \sin \left(t_{1}\right) & e^{-t_{1} p} \cos \left(t_{1}\right) & 0 & 0 \\ 0 & 0 & e^{t_{1} p} \cos \left( \pm t_{1}\right) & -e^{t_{1} p} \sin \left( \pm t_{1}\right) \\ 0 & 0 & e^{t_{1} p} \sin \left( \pm t_{1}\right) & e^{t_{1} p} \cos \left( \pm t_{1}\right)\end{array}\right)$ is conjugate to $A$ and this implies the existence of a lattice. Note that we have $T A T^{-1}=\mu\left(t_{1}\right)$ with $T:=\left(\begin{array}{cccc}\frac{1}{\sqrt{5}} & \frac{5-3 \sqrt{5}}{10} & 0 & 0 \\ 0 & 0 & \frac{1}{\sqrt{5}} & \frac{5-3 \sqrt{5}}{10} \\ -\frac{1}{\sqrt{5}} & \frac{5+3 \sqrt{5}}{10} & 0 & 0 \\ 0 & 0 & -\frac{1}{\sqrt{5}} & \frac{5+3 \sqrt{5}}{10}\end{array}\right)$.

Remark. The abelianisation of the lattice in the last proof is $\mathbb{Z} \oplus \mathbb{Z}_{3}^{2}$, i.e. the corresponding solvmanifold has $b_{1}=1$.

Proposition 7.2.13. There exists $r \in \mathbb{R} \backslash\{0, \pm 1\}$ such that $G_{5.17}^{0,0, r}$ admits a lattice.

Proof. Let $r \in\{2,3\}$. Then $\mu(t)=\left(\begin{array}{cccc}\cos (t) & \sin (t) & 0 & 0 \\ \sin (t) & \cos (t) & 0 & 0 \\ 0 & 0 & \cos (t r) & \sin (t r) \\ 0 & 0 & \sin (t r) & \cos (t r)\end{array}\right)$ is an integer matrix for $t=\pi$. This implies the existence of a lattice.

Remark. If we chose in the last proof $r=2$, then the corresponding solvmanifold has $b_{1}=3$. For $r=3$ we obtain a solvmanifold with $b_{1}=1$.

Proposition 7.2.14. $G_{5.17}^{0,0, \pm 1}$ admits a lattice.

Proof. $\mu(t)=\left(\begin{array}{cccc}\cos (t) & \sin (t) & 0 & 0 \\ \sin (t) & \cos (t) & 0 & 0 \\ 0 & 0 & \cos ( \pm t) & \sin ( \pm t) \\ 0 & 0 & \sin ( \pm t) & \cos ( \pm t)\end{array}\right)$ is an integer matrix for $t=\pi$. This implies the existence of a lattice.

Remark. The first Betti number of the solvmanifold induced by the lattice of the last proof equals one.

Proposition 7.2.15. $G_{5.18}^{0}$ admits a lattice.

Proof. Again, we follow [28]. The matrix $\left(\begin{array}{cccc}2 & 0 & 0 & -9 \\ 1 & 0 & 0 & -4 \\ 0 & 1 & 0 & -3 \\ 0 & 0 & 1 & 0\end{array}\right)$ is conjugate to

$\mu\left(t_{1}\right)=\left(\begin{array}{cccc}\cos \left(t_{1}\right) & -\sin \left(t_{1}\right) & -t_{1} \cos \left(t_{1}\right) & t_{1} \sin \left(t_{1}\right) \\ \sin \left(t_{1}\right) & \cos \left(t_{1}\right) & -t_{1} \sin \left(t_{1}\right) & -t_{1} \cos \left(t_{1}\right) \\ 0 & 0 & \cos \left(t_{1}\right) & -\sin \left(t_{1}\right) \\ 0 & 0 & \sin \left(t_{1}\right) & \cos \left(t_{1}\right)\end{array}\right)$ for $t_{1}=\frac{\pi}{3}$. This implies

the existence of a lattice. 
Note, $T=\left(\begin{array}{cccc}\frac{4}{3 \sqrt{3}} & -\frac{2}{\sqrt{3}} & 0 & -\frac{1}{\sqrt{3}} \\ 0 & 0 & 0 & 1 \\ \frac{\sqrt{3}}{\pi} & -\frac{2 \sqrt{3}}{\pi} & -\frac{\sqrt{3}}{\pi} & \frac{\sqrt{3}}{\pi} \\ \frac{1}{\pi} & 0 & -\frac{3}{\pi} & -\frac{3}{\pi}\end{array}\right) \in \mathrm{GL}(4, \mathbb{R})$ is the transformation matrix with $T A T^{-1}=\mu\left(t_{1}\right)$.

Remark. The abelianisation of the lattice in the last proof is isomorphic to $\mathbb{Z}$, i.e. the corresponding solvmanifold has $b_{1}=1$.

Algebras with nilradical $\mathfrak{n}:=\mathfrak{g}_{3.1} \oplus \mathfrak{g}_{1}=\left\langle X_{1}, \ldots, X_{4} \mid\left[X_{2}, X_{3}\right]=X_{1}\right\rangle$

We now regard the unimodular almost-nilpotent Lie groups $G_{5 . i}$ with nilradical $N:=U_{3}(\mathbb{R}) \times \mathbb{R}$, i.e. $i \in\{19,20,23,25,26,28\}$. We can identify $N$ with $\mathbb{R}^{4}$ as a manifold and the group law given by

$$
(a, b, c, r) \cdot(x, y, z, w)=(a+x+b z, b+y, c+z, r+w) .
$$

The Lie algebras of the unimodular Lie groups $G_{5 . i}=\mathbb{R} \ltimes_{\mu_{i}} N$ with nilradical $N$ are listed in Table 12. We have $\mu_{i}(t)=\exp ^{N} \circ \exp ^{A(\mathfrak{n})}\left(t \operatorname{ad}\left(X_{5}\right)\right) \circ \log ^{N}$, where $X_{5}$ depends on $i$.

Assume there is a lattice $\Gamma$ in $G_{5 . i}$. By Corollary 4.5, there are $t_{1} \neq 0$ and an inner automorphism $I_{n_{1}}$ of $N$ such that $\nu_{i}:=\mu_{i}\left(t_{1}\right) \circ I_{n_{1}}, \nu_{i}^{-1} \in \mathrm{A}(N)$ preserve the lattice $\Gamma_{N}:=\Gamma \cap N$ in $N$. For $n_{1}=(a, b, c, r)$ one calculates

$$
I_{n_{1}}(x, y, z, w)=(x+b z-y c, y, z, w) .
$$

$\Gamma_{N^{\prime}}:=\Gamma_{N} \cap N^{\prime} \cong \mathbb{Z}$ is a lattice in $N^{\prime}:=[N, N]=\{(x, 0,0,0) \mid x \in \mathbb{R}\} \cong \mathbb{R}$ by Theorem 2.4 and since $\nu_{i}\left(\Gamma_{N^{\prime}}\right), \nu_{i}^{-1}\left(\Gamma_{N^{\prime}}\right) \subset \Gamma_{N^{\prime}}$, we have $\left.\nu_{i}\right|_{\Gamma_{N^{\prime}}} \in \operatorname{Aut}(\mathbb{Z})$. This implies $\left.\nu_{i}\right|_{\Gamma_{N^{\prime}}}= \pm \mathrm{id}$ and hence $\left.\mu_{i}\left(t_{1}\right)\right|_{[N, N]}= \pm \mathrm{id}$ (a cause of (8) and the shape of $[N, N])$. Moreover, we have $[\mathfrak{n}, \mathfrak{n}]=\left\langle X_{1}\right\rangle$ and since $\exp ^{\mathbb{R}}$ is the identity,

$$
\pm \mathrm{id}=\left.\mu_{i}\left(t_{1}\right)\right|_{[N, N]}=\left.\exp ^{A(\mathfrak{n})}\left(\left.t_{1} \operatorname{ad}\left(X_{5}\right)\right|_{\left\langle X_{1}\right\rangle}\right)\right|_{[N, N]}
$$

$\left(\right.$ Note that $\exp ^{N}([\mathfrak{n}, \mathfrak{n}])=[N, N]$ by [59, Theorem 3.6.2].) Therefore, $t_{1}\left[X_{5}, X_{1}\right]$ has no component in $\left\langle X_{1}\right\rangle$ and since $t_{1} \neq 0$, this means that $\left[X_{1}, X_{5}\right]$ has no component in $X_{1}$-direction. The list of Lie algebras in Table 12 implies:

Proposition 7.2.16. The only connected and simply-connected solvable Lie groups with nilradical $U_{3}(\mathbb{R}) \times \mathbb{R}$ that can contain a lattice are $G_{5.20}^{-1}$ and $G_{5.26}^{0, \pm 1}$.

Proposition 7.2.17. The completely solvable Lie group $G_{5.20}^{-1}$ admits a lattice. For each lattice the corresponding solvmanifold admits a contact form, is formal and has $b_{1}=2$. 
Proof. Using Theorem 2.1, one shows that

$$
\begin{aligned}
\gamma_{1} & :=\left(\frac{20+9 \sqrt{5}}{9+4 \sqrt{5}}, 0,0,0\right), \\
\gamma_{2} & :=\left(\frac{181+81 \sqrt{5}}{47+21 \sqrt{5}}, \frac{18+8 \sqrt{5}}{7+3 \sqrt{5}}, \frac{2}{3+\sqrt{5}}, 0\right), \\
\gamma_{3} & :=\left(\frac{181+81 \sqrt{5}}{47+21 \sqrt{5}}, 1,1,0\right), \\
\gamma_{4} & :=\left(0,0,0,-\frac{20+9 \sqrt{5}}{(9+4 \sqrt{5}) \ln \left(\frac{3+\sqrt{5}}{2}\right)}\right)
\end{aligned}
$$

generate a lattice $\Gamma_{N}$ in $N$ with $\left[\gamma_{2}, \gamma_{3}\right]=\gamma_{1}$ and $\gamma_{1}, \gamma_{4}$ central.

A short calculation yields that $\mu(t)((x, y, z, w))=\left(x-t w, e^{-t} y, e^{t} z, w\right)$ defines a one-parameter group in $\mathrm{A}(N)$. Moreover, for $t_{1}=\ln \left(\frac{3+\sqrt{5}}{2}\right)$ holds $\mu\left(t_{1}\right)\left(\gamma_{1}\right)=\gamma_{1}$, $\mu\left(t_{1}\right)\left(\gamma_{2}\right)=\gamma_{3}, \mu\left(t_{1}\right)\left(\gamma_{3}\right)=\gamma_{2}^{-1} \gamma_{3}^{3}$ and $\mu\left(t_{1}\right)\left(\gamma_{4}\right)=\gamma_{1} \gamma_{4}$.

This implies the existence of a lattice in $G:=G_{5.20}^{-1}=\mathbb{R} \ltimes_{\mu} N$.

Let $\Gamma$ be an arbitrary lattice in $G$. By completely solvability and Theorem 3.11 (ii), we get the minimal model of $M=G / \Gamma$ as the minimal model $\mathcal{M}$ of the Chevalley-Eilenberg complex $\left(\bigwedge \mathfrak{g}^{*}, \delta\right)$. The latter is given by

$$
\delta x_{1}=-x_{23}-x_{45}, \delta x_{2}=-x_{25}, \delta x_{3}=x_{35}, \delta x_{4}=\delta x_{5}=0,
$$

which implies $b_{1}(M)=2$. Moreover, $x_{1}$ defines a left-invariant contact form on $G / \Gamma$.

One computes the differential of the non-exact generators of degree two in the Chevalley-Eilenberg complex as

$$
\begin{array}{ccl}
\delta x_{12}=x_{125}-x_{245}, & \delta x_{13}=-x_{135}-x_{345}, & \delta x_{14}=-x_{234}, \\
\delta x_{15}=-x_{235}, & \delta x_{23}=0, & \delta x_{24}=x_{245}, \\
\delta x_{34}=-x_{345}, & \delta x_{45}=0 . &
\end{array}
$$

The minimal model $\rho:(\bigwedge V, d) \rightarrow\left(\bigwedge \mathfrak{g}^{*}, \delta\right)$ must contain two closed generators $y_{1}, y_{2}$ which map to $x_{4}$ and $x_{5}$. We see $\rho\left(y_{1} y_{2}\right)=x_{45}$ is closed and non-exact. Since $b_{2}(G / \Gamma)=1$, the minimal model's construction in the proof of Theorem 1.1.2 implies that there are no further generators of degree less than or equal to two in $V$. This implies that $G / \Gamma$ is formal.

Proposition 7.2.18. $G_{5.26}^{0, \varepsilon}$ admits a lattice for $\varepsilon= \pm 1$. For each lattice the corresponding solvmanifold is contact and has $b_{1} \geq 2$.

Proof. One calculates that $\mu: \mathbb{R} \rightarrow \mathrm{A}(N)$ defined by 


$$
\begin{aligned}
& \mu(t)((x, y, z, w)) \\
& \quad=\left(x+h_{t}(y, z)-\varepsilon t w, \cos (t \pi) y-\sin (t \pi) z, \sin (t \pi) y+\cos (t \pi) z, w\right),
\end{aligned}
$$

where $h_{t}(y, z)=\frac{1}{2} \sin (t \pi)\left(\cos (t \pi)\left(y^{2}-z^{2}\right)-2 \sin (t \pi) y z\right)$, is a one-parameter group.

Then we have $G:=G_{5.26}^{0, \varepsilon}=\mathbb{R} \ltimes_{\mu} N$ and $\mathbb{Z} \ltimes_{\mu}\{(x, y, z, w) \in N \mid x, y, z, w \in \mathbb{Z}\}$ is a lattice in $G$ since $\mu(1)((x, y, z, w))=(x-\varepsilon w,-y,-z, w)$.

Using $d_{e}(\mu(t))=\log ^{N} \circ \mu(t) \circ \exp ^{N}$, we obtain the Lie algebra $\mathfrak{g}$ of $G$ as

$$
\left\langle X_{1}, \ldots X_{5} \mid\left[X_{2}, X_{3}\right]=X_{1},\left[X_{2}, X_{5}\right]=X_{3},\left[X_{3}, X_{5}\right]=-X_{2},\left[X_{4}, X_{5}\right]=\varepsilon X_{1}\right\rangle .
$$

Denote $\left\{x_{1}, \ldots, x_{5}\right\}$ the basis of $\mathfrak{g}^{*}$ which is dual to $\left\{X_{1}, \ldots, X_{5}\right\}$, i.e. the $x_{i}$ are left-invariant 1-forms on $G$. One calculates that $x_{1}$ is a left-invariant contact form on $G$, so it descends to a contact form on the corresponding solvmanifold.

The statement about the first Betti number follows from Theorem 3.11(i).

Remark. Since the abelianisation of the lattice in the last proof is isomorphic to $\mathbb{Z}^{2} \oplus \mathbb{Z}_{2}^{2}$, the corresponding solvmanifold has $b_{1}=2$.

\section{Algebras with nilradical $\mathfrak{g}_{4.1}=\left\langle X_{1}, \ldots, X_{4} \mid\left[X_{2}, X_{4}\right]=X_{1},\left[X_{3}, X_{4}\right]=X_{2}\right\rangle$}

Proposition 7.2.19. No connected and simply-connected solvable Lie group $G_{5 . i}$ with nilradical $N:=G_{4.1}$ admits a lattice.

Proof. There is only one unimodular connected and simply-connected solvable Lie group with nilradical $G_{4.1}$, namely the completely solvable group $G:=G_{5.30}^{-\frac{4}{3}}$. We show that it admits no lattice.

The group $N$ is $\mathbb{R}^{4}$ as a manifold with multiplication given by

$$
(a, b, c, r) \cdot(x, y, z, w)=\left(a+x+w b+\frac{1}{2} w^{2} c, b+y+w c, c+z, r+w\right),
$$

and one calculates for $n_{1}=(a, b, c, r)$

$$
I_{n_{1}}(x, y, z, w)=\left(x+w b+\frac{1}{2} w^{2} c-r y-r w c+\frac{1}{2} r^{2} z, y+w c-r z, z, w\right) .
$$

Let $G=\mathbb{R} \ltimes_{\mu} N$, where $\mu(t)=\exp ^{N} \circ \exp ^{A(\mathfrak{n})}\left(t \operatorname{ad}\left(X_{5}\right)\right) \circ \log ^{N}$ and assume there is a lattice $\Gamma$ in $G$. By Corollary 4.5, there are $t_{1} \neq 0$ and $n_{1} \in N$ such that $\nu:=\mu\left(t_{1}\right) \circ I_{n_{1}} \in \mathrm{A}(N)$ preserves the lattice $\Gamma_{N}:=\Gamma \cap N$ in $N$.

$\Gamma_{N^{\prime}}:=N^{\prime} \cap \Gamma_{N}$ is a lattice in $N^{\prime}:=[N, N]=\{(x, y, 0,0) \in N \mid x, y \in \mathbb{R}\} \cong \mathbb{R}^{2}$ by Theorem 2.4, and since $\nu\left(N^{\prime}\right) \subset N^{\prime}$, this lattice is preserved by $\left.\nu\right|_{N^{\prime}}$. This and $\exp ^{\mathbb{R}^{2}}=$ id imply

$$
\pm 1=\operatorname{det}\left(\left.\nu\right|_{N^{\prime}}\right)=\operatorname{det}\left(\left.\exp ^{A(\mathfrak{n})}\left(\left.t_{1} \operatorname{ad}\left(X_{5}\right)\right|_{[\mathfrak{n}, \mathfrak{n}]}\right)\right|_{[N, N]}\right) \cdot \underbrace{\operatorname{det}\left(\left.I_{n_{1}}\right|_{N^{\prime}}\right)}_{=1},
$$

i.e. $\left.\operatorname{ad}\left(X_{5}\right)\right|_{[\mathfrak{n}, \mathfrak{n}]}$ has trace equal to zero. This contradicts $\mathfrak{g}_{5.30}^{-\frac{4}{3}}$, see Table 13 , 


\section{Non-almost nilpotent algebras}

Now, there remain two unimodular connected and simply-connected solvable Lie groups in dimension five, namely $G_{5.33}^{-1,-1}$ and $G_{5.35}^{-2,0}$. Unfortunately, we do not know whether the former group admits a lattice or not. Note, Harshavardhan's argumentation in [28, p. 33] is not sufficient.

Remark. If the completely solvable Lie group $G_{5.33}^{-1,-1}$ admits a lattice, one easily proves that the corresponding solvmanifold admits a contact form (since $G_{5.33}^{-1,-1}$ possesses the left-invariant contact form $x_{1}+x_{2}+x_{3}$ with $x_{i}$ dual to $X_{i} \in \mathfrak{g}_{5.33}^{-1,-1}$ as in Table 14), is formal and has $b_{1}=2$.

Remark. In April 2009, A. Diatta and B. Foreman proved that $G_{5.33}^{-1,-1}$ possesses a lattice.

Proposition 7.2.20. $G_{5.35}^{-2,0}$ contains a lattice. For each lattice the corresponding solvmanifold is contact and has $b_{1} \geq 2$.

Proof. A lattice and a contact form were constructed by Geiges in [25]. One has the left-invariant contact form $x_{1}+x_{2}$ on the Lie group, where $x_{1}, x_{2}$ are dual to the left-invariant vector fields as in Table 14. Hence the form descends to each compact quotient by a discrete subgroup.

The statement about the first Betti number follows from Theorem 3.11(i).

\section{Conclusion}

We have seen that each connected and simply-connected 5-dimensional solvable Lie group admits a lattice if it is nilpotent or decomposable with the exception of $G_{4.2} \times \mathbb{R}$. If an indecomposable non-nilpotent group $G_{5 . i}$ gives rise to a solvmanifold it is contained in Table 3, Recall, by Theorem 3.11, we always have a lower bound for the solvmanifold's Betti numbers and in some cases the exact value. These can be read of in the second and the third column. The last column refers to the examples that we have constructed above. "yes" means that we have such for certain parameters that satisfy the conditions of the column "Comment". Except for $i=33$ we have examples for all possible values of $i$.

Assuming that there is a lattice in one the non-completely solvable Lie groups $G_{5 . i}$, i.e. $i \in\{13,14,17,18,26,35\}$, such that the inequalities in the above table are equalities, then one can calculate that such quotients are formal for $i \in$ $\{13,17,26,35\}$ and not formal for $i \in\{14,18\}$. The assumptions about the Betti numbers are needed to ensure that the Lie algebra cohomology is isomorphic to the solvmanifold's cohomology.

\subsection{Contact structures}

Some of the connected and simply-connected five-dimensional solvable Lie groups $G_{5 . i}$ which admit a lattice $\Gamma$ possess a left-invariant contact form. Obviously, it 
Table 3: 5-dimensional indecomposable non-nilmanifolds

\begin{tabular}{|c|c|c|c|c|c|}
\hline \hline & $b_{1}$ & $b_{2}$ & formal & Comment & Example \\
\hline \hline$G_{5.7}^{p, q, r}$ & 1 & 0 & yes & $\begin{array}{c}-1<r<p<q<1, \\
p q r \neq 0, \\
p+q+r=-1\end{array}$ & 7.2.1 (i) \\
\hline$G_{5,7}^{p, q,-1}$ & 1 & 2 & yes & $p=-q \in] 0,1[$ & $7.2 .1(\mathrm{ii})$ \\
\hline$G_{5.7}^{1,-1,-1}$ & 1 & 4 & yes & & $7.2 .1(\mathrm{iii})$ \\
\hline$G_{5.8}^{-1}$ & 2 & 3 & no & & 7.2 .2 \\
\hline$G_{5.13}^{-1-2 q, q, r}$ & $\geq 1$ & $\geq 0$ & $?$ & $q \in[-1,0] \backslash\left\{\frac{1}{2}\right\}$, & 7.2 .5 \\
\hline$G_{5.13}^{-1,0, r}$ & $\geq 1$ & $\geq 2$ & $?$ & $r \neq 0$ & \\
\hline$G_{5.14}^{0}$ & $\geq 2$ & $\geq 3$ & $?$ & & 7.2 .6 \\
\hline$G_{5.15}^{-1}$ & 1 & 2 & no & & 7.2 .7 \\
\hline$G_{5.17}^{p,-p}$ & $\geq 1$ & $\geq 0$ & $?$ & $p \neq 0, r \notin\{0, \pm 1\}$ & 7.2 .9 \\
\hline$G_{5.17}^{p,-p, \pm 1}$ & $\geq 1$ & $\geq 2$ & $?$ & $p \neq 0$ & 7.2 .11 \\
\hline$G_{5.17}^{0,0, r}$ & $\geq 1$ & $\geq 2$ & $?$ & $r \notin\{0, \pm 1\}$ & 7.2 .13 \\
\hline$G_{5.17}^{0,0, \pm 1}$ & $\geq 1$ & $\geq 4$ & $?$ & & 7.2 .14 \\
\hline$G_{5.18}^{0}$ & $\geq 1$ & $\geq 2$ & $?$ & & 7.2 .15 \\
\hline$G_{5.20}^{-1}$ & 2 & 1 & yes & & 7.2 .17 \\
\hline$G_{5.26}^{0, \pm 1}$ & $\geq 2$ & $\geq 1$ & $?$ & & 7.2 .18 \\
\hline$G_{5.33}^{-1,-1}$ & 2 & 1 & yes & & no \\
\hline$G_{5.35}^{-2,0}$ & $\geq 2$ & $\geq 1$ & $?$ & & 7.2 .20 \\
\hline
\end{tabular}

also defines a contact form on the corresponding solvmanifold. By this way, we showed that the manifolds $G_{5 . i} / \Gamma$ for $i \in\{4,5,6\}$ and quotients of almost nilpotent groups with non-abelian nilradical (i.e. $i \geq 19$ ) by lattices are contact.

But $\mathbb{R}^{5}, U_{3}(\mathbb{R}) \times \mathbb{R}^{2}, G_{4.1} \times \mathbb{R}$ and $G_{5 . i}$ do not have a left-invariant contact form for $i \in\{1,2,3,7, \ldots, 18\}$, see e.g. [15]. For some of the nilmanifolds, we can provide a contact structure by another approach.

Theorem 7.3.1. Let $G \in\left\{\mathbb{R}^{5}, U_{3}(\mathbb{R}) \times \mathbb{R}^{2}, G_{4.1} \times \mathbb{R}, G_{5.1}, G_{5.3}\right\}$ and $\Gamma$ a lattice $G$. Then $G / \Gamma$ admits a contact structure.

Proof. For $G$ chosen as in the theorem, the dimension of the center is greater than or equal to two. Therefore, we can find a two-dimensional closed normal subgroup that lies in the center such that its intersection with $\Gamma$ is a lattice in it. By Theorem 3.6, $G / \Gamma$ has the structure of a principal $T^{2}$-bundle over a three dimensional closed orientable manifold. Then the following result of Lutz implies the claim.

Theorem 7.3.2 ([37]). The total space of a principal $T^{2}$-bundle over a closed orientable 3-manifold admits a contact form. 
Unfortunately, we did not find a contact structure on the manifold of Proposition 7.2.9. If such exists, this yields a five-dimensional non-formal contact solvmanifold with $b_{1}=1$.

\section{Six-dimensional solvmanifolds}

There are 164 types of connected and simply-connected indecomposable solvable Lie groups in dimension six, most of them depending on parameters. For classifying six-dimensional solvmanifolds, we restrict ourselves to the following types:

(1) nilmanifolds

(2) symplectic solvmanifolds that are quotients of indecomposable non-nilpotent groups

(3) products of lower-dimensional solvmanifolds

Although we have to make some restrictions to get a manageable number of cases, one certainly has to consider types (1) and (3). Concerning the third type, the reader can even ask the legitimate question why we do not consider arbitrary lattices in products of lower dimensional solvable Lie groups $G_{1}, G_{2}$, instead of direct products $\Gamma_{1} \times \Gamma_{2}$ of lattices $\Gamma_{i}$ in the factors $G_{i}$. The reason is that we have no tool to construct arbitrary lattices or disprove their existence, unless we can ensure that they contain the semidirect factor $\mathbb{Z}$. (When we wanted to investigate $G_{5.33}^{-1,-1}$, we already had this problem.)

The further restriction in (2) is justified by the large number of indecomposable non-nilpotent solvable Lie algebras in dimension six: There are 140 types of it. The author has decided to consider the most interesting among them. Since we are not able to refute a symplectic form's existence in the non-completely solvable case, we shall partly make even more restrictions.

\subsection{Nilmanifolds}

There are 34 isomorphism classes of nilpotent Lie algebras in dimension six. Each of them possesses a basis with rational structure constants and therefore determines a nilmanifold. They are listed on page 52 in Table 4 which is taken from [52. The corresponding Lie algebras are listed in Appendix A, Among the 34 classes of nilmanifolds, there are 26 which admit a symplectic form.

Recall that a nilmanifold is formal or Kählerian if and only if the corresponding Lie algebra is abelian. 
Table 4: 6-dimensional nilmanifolds

\begin{tabular}{|c|c|c|c|}
\hline$\overline{b_{1}(G / \Gamma)}$ & $\overline{b_{2}(G / \Gamma)}$ & Comment & $\mathfrak{g}$ \\
\hline 6 & 15 & "Torus, symplectic & $6 \mathfrak{g}_{1}$ \\
\hline 5 & 11 & symplectic & $\mathfrak{g}_{3.1} \oplus 3 \mathfrak{g}_{1}$ \\
\hline 5 & 9 & not symplectic & $\overline{\mathfrak{g}_{5.4} \oplus \mathfrak{g}_{1}}$ \\
\hline$\overline{4}$ & $\overline{99}$ & symplectic & $\mathfrak{g}_{5.1} \oplus \mathfrak{g}_{1}$ \\
\hline 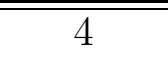 & 8 & symplectic & $2 \mathfrak{g}_{3.1}$ \\
\hline 4 & 8 & symplectic & $\mathfrak{g}_{6 . N 4}$ \\
\hline 4 & 8 & symplectic & $\mathfrak{g}_{6 . N 5}$ \\
\hline$\overline{44}$ & 7 & symplectic & $\mathfrak{g}_{5.5} \oplus \mathfrak{g}_{1}$ \\
\hline 4 & 7 & symplectic & $\mathfrak{g}_{4.1} \oplus 2 \mathfrak{g}_{1}$ \\
\hline 4 & 6 & not symplectic & $\mathfrak{g}_{6 . N 12}$ \\
\hline 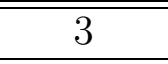 & 8 & symplectic & $\mathfrak{g}_{6 . N 3}$ \\
\hline 3 & 6 & symplectic & $\overline{\mathfrak{g}_{6 . N 1}}$ \\
\hline 3 & 6 & symplectic & $\mathfrak{g}_{6 . N 6}$ \\
\hline 3 & 6 & symplectic & $\mathfrak{g}_{6 . N 7}$ \\
\hline 3 & 5 & symplectic & $\mathfrak{g}_{5.2} \oplus \mathfrak{g}_{1}$ \\
\hline 3 & 5 & not symplectic & $\mathfrak{g}_{5.3} \oplus \mathfrak{g}_{1}$ \\
\hline 3 & 5 & symplectic & $\mathfrak{g}_{5.6} \oplus \mathfrak{g}_{1}$ \\
\hline 3 & 5 & symplectic & $\mathfrak{g}_{6 . N 8}$ \\
\hline 3 & 5 & symplectic & $\mathfrak{g}_{6 . N 9}$ \\
\hline 3 & 5 & symplectic & $\mathfrak{g}_{6 . N 10}$ \\
\hline 3 & 5 & not symplectic & $\mathfrak{g}_{6 . N 13}$ \\
\hline 3 & 5 & not symplectic & $\mathfrak{g}_{6 . N 14}^{1}$ \\
\hline 3 & 5 & not symplectic & $\mathfrak{g}_{6 . N 14}^{-1}$ \\
\hline 3 & 5 & symplectic & $\mathfrak{g}_{6 . N 15}$ \\
\hline 3 & 5 & symplectic & $\mathfrak{g}_{6 . N 17}$ \\
\hline 3 & 4 & symplectic & $\begin{array}{l}\mathfrak{g}_{6 . N 16} \\
\end{array}$ \\
\hline 2 & 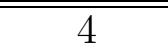 & symplectic & 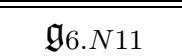 \\
\hline 2 & 4 & symplectic & $\mathfrak{g}_{6 . N 18}^{1}$ \\
\hline 2 & 4 & symplectic & $\mathfrak{g}_{6 . N 18}^{-1}$ \\
\hline 2 & 3 & symplectic & $\mathfrak{g}_{6 . N 2}$ \\
\hline 2 & 3 & symplectic & $\mathfrak{g}_{6 . N 19}$ \\
\hline 2 & 3 & symplectic & $\mathfrak{g}_{6 . N 20}$ \\
\hline 2 & 2 & not symplectic & $\begin{array}{l}\mathfrak{g}_{6 . N 21} \\
\end{array}$ \\
\hline 2 & 2 & not symplectic & $\mathfrak{g}_{6 . N 22}$ \\
\hline
\end{tabular}




\subsection{Candidates for the existence of lattices}

Among the 61 types of indecomposable unimodular almost nilpotent Lie algebras in dimension six that are listed in Tables 17- 30, there are some that cannot be the Lie algebra of a connected and simply-connected Lie group which admits a lattice.

Instead of the small German letters for the Lie algebras in the mentioned tables, we use again capital Latin letters with the same subscripts for the corresponding connected and simply-connected Lie groups. If any, we chose the same designation for the parameters $a, b, c, h, s, \varepsilon$ of $G_{6 . i}$ as for their Lie algebras.

Proposition 8.2.1. Let $i \in\{13, \ldots, 38\}$, i.e. $\operatorname{Nil}\left(G_{6 . i}\right)=U_{3}(\mathbb{R}) \times \mathbb{R}^{2}$. Then it is necessary for $G_{6 . i}$ to contain a lattice that one of the following conditions holds:

$$
\begin{aligned}
& i=15, \quad i=18 \wedge a=-1, \quad i=21 \wedge a=0, \quad i=23 \wedge a=0, \\
& i=25 \wedge b=0, \quad i=26, \quad i=29 \wedge b=0, \quad i=32 \wedge a=0, \\
& i=33 \wedge a=0, \quad i=34 \wedge a=0, \quad i=35 \wedge a=-b, \quad i=36 \wedge a=0, \\
& i=37 \wedge a=0, \quad i=38 \text {. }
\end{aligned}
$$

Proof. This can be seen analogous as in the proof of Proposition 7.2.16. Denote $\left\{X_{1}, \ldots, X_{6}\right\}$ the basis used for the description of the Lie algebra in Appendix $\mathrm{A}$. Then the existence of a lattice implies that $\left[X_{6}, X_{1}\right]$ has no component in $X_{1}$-direction and this yields the claim.

Proposition 8.2.2. Let $i \in\{39, \ldots, 47\}$, i.e. the nilradical of $G_{6 . i}$ is $G_{4.1} \times \mathbb{R}$. If $G_{6 . i}$ admits a lattice, then holds $i=39 \wedge h=-3$ or $i=40$.

Proof. Use the designation $X_{1}, \ldots, X_{6}$ as above. Then $\left\langle X_{1}, X_{2}\right\rangle$ is the commutator of the nilradical of $\mathfrak{g}_{6 . i}$. Analogous as in the proof of Proposition 7.2.19, one shows that $\left.\operatorname{ad}\left(X_{6}\right)\right|_{\left\langle X_{1}, X_{2}\right\rangle}$ has trace equal to zero. This is only satisfied for $i=39 \wedge h=-3$ or $i=40$.

\section{Proposition 8.2.3.}

(i) Let $i \in\{54, \ldots, 70\}$, i.e. the nilradical of $G_{6 . i}$ is $G_{5.1}$. If $G_{6 . i}$ admits a lattice, then holds $i=54 \wedge l=-1, i=63, i=65 \wedge l=0$ or $i=70 \wedge p=0$.

(ii) No connected and simply-connected almost nilpotent Lie group with nilradical $G_{5.2}$ or $G_{5.5}$ admits a lattice.

Proof. This follows in the same manner as the last proposition. The trace of $\operatorname{ad}\left(X_{6}\right)$ restricted to the commutator of the nilradical must be zero. 


\subsection{Symplectic solvmanifolds whose first Betti number equals one}

If we are looking for solvmanifolds with $b_{1}=1$, it is necessary that the corresponding Lie algebra is unimodular, almost nilpotent and has $b_{1}=1$ itself. Note that the latter forces the algebra to be indecomposable. In Tables 34 - 36] on pages 94 - 96 we have listed all possible values that can arise as $b_{1}$ for the classes of unimodular non-nilpotent solvable indecomposable Lie algebras in dimension six.

Since we are mainly interested in symplectic 6-manifolds, we now investigate which Lie algebras contained in Tables $18-30$ that satisfy $b_{1}=1$ are cohomologically symplectic, i.e. there is a closed element $\omega \in \bigwedge^{2} \mathfrak{g}^{*}$ such that $\omega^{3}$ is not exact.

Note, if a unimodular Lie algebra is cohomologically symplectic, then each compact quotient of the corresponding Lie group by a lattice is symplectic. If the Lie algebra is completely solvable, this is even necessary for the quotient to be symplectic.

Proposition 8.3.1. Let $\mathfrak{g}_{6 . i}$ be a unimodular almost-nilpotent Lie algebra with $b_{1}\left(\mathfrak{g}_{6 . i}\right)=1$. Then we have:

$\mathfrak{g}_{6 . i}$ is cohomologically symplectic if and only if $i \in\{15,38,78\}$.

Proof. For $i \in\{15,38,78\}$ one computes all symplectic forms up to exact summands as

$$
\begin{aligned}
& i=15: \omega=(\lambda+\mu) x_{16}+\lambda x_{25}-\mu x_{34}, \quad \lambda, \mu \in \mathbb{R} \backslash\{0\}, \lambda \neq-\mu, \\
& i=38: \omega=\lambda x_{16}+\mu x_{24}+\frac{\lambda}{2} x_{25}-\frac{\lambda}{2} x_{34}+\mu x_{35}, \quad \lambda, \mu \in \mathbb{R}, \lambda \neq 0,-\frac{3}{2} \lambda^{3} \neq 2 \lambda \mu^{2}, \\
& i=78: \omega=\lambda x_{14}+\lambda x_{26}+\lambda x_{35}, \quad \lambda \in \mathbb{R} \backslash\{0\} .
\end{aligned}
$$

If $i \notin\{15,38,78\}$, then the conditions on the parameters of $\mathfrak{g}_{6 . i}$ to ensure its unimodularity and $b_{1}\left(\mathfrak{g}_{6 . i}\right)=1$ imply that there are no closed elements of $\bigwedge^{2} \mathfrak{g}_{6 . i}^{*}$ without exact summands which contain one of the elements $x_{16}, x_{26}, x_{36}, x_{46}$ or $x_{56}$. Therefore, $\mathfrak{g}_{6 . i}$ cannot be cohomologically symplectic.

Remark. We give an explicit example of the argumentation in the last proof for $i=2$ :

$\mathfrak{g}_{6.2}$ depends on three parameters $a, c, d \in \mathbb{R}$ with $0<|d| \leq|c| \leq 1$ and the brackets are given in Table 18 as

$$
\begin{aligned}
& {\left[X_{1}, X_{6}\right]=a X_{1}, \quad\left[X_{2}, X_{6}\right]=X_{1}+a X_{2}, \quad\left[X_{3}, X_{6}\right]=X_{3}} \\
& {\left[X_{4}, X_{6}\right]=c X_{4}, \quad\left[X_{5}, X_{6}\right]=d X_{5} .}
\end{aligned}
$$

The condition of unimodularity implies $2 a+c+d=-1$. Moreover, if first the Betti number equals one, we see in Tabular 34 that $a \neq 0$. 
The Chevalley-Eilenberg complex is given by

$$
\begin{array}{lll}
\delta x_{1}=-a x_{16}-x_{26}, & \delta x_{2}=-a x_{26}, & \delta x_{3}=-x_{36} \\
\delta x_{4}=-c x_{46}, & \delta x_{5}=-d x_{56}, & \delta x_{6}=0
\end{array}
$$

and since $a, c, d \neq 0, x_{26}, x_{36}, x_{46}, x_{56}$ are exact. Moreover, $x_{16}=\delta\left(-\frac{1}{a} x_{1}+\frac{1}{a^{2}} x_{2}\right)$ is exact, too. This implies the claim.

We now examine the three Lie groups that have cohomologically symplectic Lie algebras.

The next theorem was announced in [6]. It provides an example of a symplectic non-formal 6 -manifold with $b_{1}=1$. Since it is a solvmanifold, this manifold is symplectically aspherical. Hence, we found an example for which Kędra, Rudyak and Tralle looked in [35, Remark 6.5].

\section{Theorem 8.3.2.}

(i) The completely solvable Lie group $G_{6.15}^{-1}$ contains a lattice.

(ii) If $\Gamma$ is any lattice in $G:=G_{6.15}^{-1}$, then $M:=G / \Gamma$ is a symplectic and non-formal manifold with $b_{1}(M)=1$ and $b_{2}(M)=2$.

Proof. ad (i): Let $N=U_{3}(\mathbb{R}) \times \mathbb{R}^{2}$ denote the nilradical of $G$. We can identify $N$ with $\mathbb{R}^{5}$ as a manifold and the multiplication given by

$$
(a, b, c, r, s) \cdot(x, y, z, v, w)=(a+x+b z, b+y, c+z, r+v, s+w),
$$

i.e. $[N, N]=\{(x, 0,0,0,0) \mid x \in \mathbb{R}\} \cong \mathbb{R}$ and $\bar{N}:=N /[N, N] \cong \mathbb{R}^{4}$.

By definition of $G$, we have $G=\mathbb{R} \ltimes_{\mu} N$, where

$$
\forall_{t \in \mathbb{R}} \quad \mu(t)=\exp ^{N} \circ \exp ^{A(\mathfrak{n})}\left(t\left(\begin{array}{ccccc}
0 & 0 & 0 & 0 & 0 \\
0 & -1 & 0 & 0 & 0 \\
0 & 0 & 1 & 0 & 0 \\
0 & -1 & 0 & -1 & 0 \\
0 & 0 & -1 & 0 & 1
\end{array}\right)\right) \circ \log ^{N}
$$

and since $\exp ^{\mathbb{R}^{4}}=\mathrm{id}$, the induced maps $\bar{\mu}(t): \bar{N} \rightarrow \bar{N}$ are given by

$$
\begin{aligned}
\bar{\mu}(t)((y, z, v, w)) & =\exp ^{G L(4, \mathbb{R})}\left(t\left(\begin{array}{cccc}
-1 & 0 & 0 & 0 \\
0 & 1 & 0 & 0 \\
-1 & 0 & -1 & 0 \\
0 & -1 & 0 & 1
\end{array}\right)\right)\left(\begin{array}{c}
y \\
z \\
v \\
w
\end{array}\right) \\
& =\left(\begin{array}{cccc}
e^{-t} & 0 & 0 & 0 \\
0 & e^{t} & 0 & 0 \\
-t e^{-t} & 0 & e^{-t} & 0 \\
0 & -t e^{t} & 0 & e^{t}
\end{array}\right)\left(\begin{array}{c}
y \\
z \\
v \\
w
\end{array}\right)
\end{aligned}
$$


One calculates that $\widetilde{\mu}: \mathbb{R} \rightarrow \mathrm{A}(N)$ given by

$$
\forall_{t \in \mathbb{R}} \quad \forall_{(x, y, z, v, w) \in N} \quad \widetilde{\mu}(t)((x, y, z, v, w))=(x, \bar{\mu}(t)((y, z, v, w)))
$$

is a one-parameter group, and since the derivations of (9) and (10) in zero are equal, we have $\mu \equiv \widetilde{\mu}$.

Let $t_{1}=\ln \left(\frac{3+\sqrt{5}}{2}\right)$, then $\bar{\mu}\left(t_{1}\right)$ is conjugate to $A:=\left(\begin{array}{llll}2 & 1 & 0 & 0 \\ 1 & 1 & 0 & 0 \\ 2 & 1 & 2 & 1 \\ 1 & 1 & 1 & 1\end{array}\right)$. The transformation matrix $T \in \mathrm{GL}(4, \mathbb{R})$ with $T A T^{-1}=\bar{\mu}\left(t_{1}\right)$ is

$$
T=\left(\begin{array}{cccc}
1 & -\frac{2(2+\sqrt{5})}{3+\sqrt{5}} & 0 & 0 \\
1 & \frac{1+\sqrt{5}}{3+\sqrt{5}} & 0 & 0 \\
0 & 0 & \ln \left(\frac{2}{3+\sqrt{5}}\right) & \frac{2(2+\sqrt{5}) \ln \left(\frac{3+\sqrt{5}}{2}\right)}{3+\sqrt{5}} \\
0 & 0 & \ln \left(\frac{2}{3+\sqrt{5}}\right) & -\frac{(1+\sqrt{5}) \ln \left(\frac{3+\sqrt{5}}{2}\right)}{3+\sqrt{5}}
\end{array}\right) .
$$

Denote by $\left\{b_{1}, \ldots, b_{4}\right\}$ the basis of $\mathbb{R}^{4}$ for which $\bar{\mu}\left(t_{1}\right)$ is represented by $A$, i.e. $b_{i}$ is the $i$-th column of $T$. One calculates

$$
\begin{aligned}
b_{11} b_{22}-b_{12} b_{21} & =\sqrt{5} \\
b_{i 1} b_{j 2}-b_{i 2} b_{j 1} & =0 \quad \text { for } i<j,(i, j) \neq(1,2) .
\end{aligned}
$$

This implies that we have for $\gamma_{0}:=\left(\sqrt{5}, 0_{\mathbb{R}^{4}}\right), \gamma_{i}:=\left(b_{i 0}, b_{i}\right) \in N$ with arbitrary $b_{i 0} \in \mathbb{R}, i=1, \ldots, 4$,

$$
\left[\gamma_{1}, \gamma_{2}\right]=\gamma_{0},\left[\gamma_{1}, \gamma_{3}\right]=\left[\gamma_{1}, \gamma_{4}\right]=\left[\gamma_{2}, \gamma_{3}\right]=\left[\gamma_{2}, \gamma_{4}\right]=\left[\gamma_{3}, \gamma_{4}\right]=e_{N} .
$$

We can choose the $b_{i 0}$ such that the following equations hold:

$$
\begin{array}{lllllll}
\mu\left(t_{1}\right)\left(\gamma_{0}\right) & = & \gamma_{0}, & & & & \\
\mu\left(t_{1}\right)\left(\gamma_{1}\right) & = & & \gamma_{1}^{2} & \gamma_{2} & \gamma_{3}^{2} & \gamma_{4}, \\
\mu\left(t_{1}\right)\left(\gamma_{2}\right) & = & & \gamma_{1} & \gamma_{2} & \gamma_{3} & \gamma_{4}, \\
\mu\left(t_{1}\right)\left(\gamma_{3}\right) & = & & & & \gamma_{3}^{2} & \gamma_{4}, \\
\mu\left(t_{1}\right)\left(\gamma_{4}\right) & = & & & & \gamma_{3} & \gamma_{4} .
\end{array}
$$

Note that (11) leads to the equation $\left(\mathrm{id}-{ }^{\tau} A\right)\left(\begin{array}{c}b_{10} \\ b_{20} \\ b_{30} \\ b_{40}\end{array}\right)=\left(\begin{array}{c}1+\frac{2(1+\sqrt{5})}{3+\sqrt{5}} \\ \frac{1+\sqrt{5}}{3+\sqrt{5}} \\ 0 \\ 0\end{array}\right)$ which has the (unique) solution $b_{10}=-\frac{1+\sqrt{5}}{3+\sqrt{5}}, b_{20}=-\frac{11+5 \sqrt{5}}{7+3 \sqrt{5}}$ and $b_{30}=b_{40}=0$.

We claim that $t_{1} \mathbb{Z} \ltimes_{\mu}\left\langle\exp ^{N}\left(\operatorname{Span}_{\mathbb{Z}} \log ^{N}\left(\left\{\gamma_{0}, \ldots, \gamma_{4}\right\}\right)\right)\right\rangle$ defines a lattice in $G$ : 
It suffices to show that $\left\langle\exp ^{N}\left(\operatorname{Span}_{\mathbb{Z}} \log ^{N}\left(\left\{\gamma_{0}, \ldots, \gamma_{4}\right\}\right)\right)\right\rangle$ defines a lattice in $N$, so let us prove this assertion. There exist uniquely $Y_{0}, \ldots, Y_{4} \in \mathfrak{n}$ with $\exp ^{N}\left(Y_{i}\right)=\gamma_{i}$ for $i \in\{0, \ldots, 4\}$. If we prove that $\mathfrak{Y}:=\left\{Y_{0}, \ldots, Y_{4}\right\}$ is a basis of $\mathfrak{n}$ with rational structure constants, then Theorem 2.1 (i) implies that $\left\langle\exp ^{N}\left(\operatorname{Span}_{\mathbb{Z}} \mathfrak{Y}\right)\right\rangle$ is a lattice in $N$.

We identify $\mathfrak{n}$ with $\mathbb{R}^{5}$ and brackets given by the Campbell-Hausdorff formula, see e.g. [59, Chapter 2.15]. Since $\mathfrak{n}$ is 2-step nilpotent (and $\exp ^{N}$ is a diffeomorphism), the formula yields for all $V, W \in \mathfrak{n}$

$$
\log ^{N}\left(\exp ^{N}(V) \exp ^{N}(W)\right)=V+W+\frac{1}{2}[V, W]
$$

Since $U_{3}(\mathbb{R})$ can be considered as a group of matrices, one can easily calculate its exponential map. Then, its knowledge implies that the exponential map resp. the logarithm of $N$ is given by

$$
\begin{aligned}
& \exp ^{N}((x, y, z, v, w))=\left(x+\frac{1}{2} y z, y, z, v, w\right) \\
& \log ^{N}((x, y, z, v, w))=\left(x-\frac{1}{2} y z, y, z, v, w\right)
\end{aligned}
$$

and we obtain $Y_{0}=\left(\sqrt{5}, 0_{\mathbb{R}^{4}}\right), Y_{1}=\left(b_{10}-\frac{1}{2}, b_{1}\right), Y_{2}=\left(b_{20}+\frac{(2+\sqrt{5})(1+\sqrt{5})}{(3+\sqrt{5})^{2}}, b_{2}\right)$, $Y_{3}=\left(0, b_{3}\right), Y_{4}=\left(0, b_{4}\right),\left[Y_{1}, Y_{2}\right]=Y_{0}$. The other brackets vanish.

ad (ii): Let $\Gamma$ be an arbitrary lattice in $G$. By completely solvability and Theorem 3.11 (ii), we get the minimal model of $M=G / \Gamma$ as the minimal model $\mathcal{M}$ of the Chevalley-Eilenberg complex $\left(\bigwedge \mathfrak{g}^{*}, \delta\right)$. The latter has the closed generator $x_{6}$ and the non-closed generators satisfy

$$
\delta x_{1}=-x_{23}, \delta x_{2}=-x_{26}, \delta x_{3}=x_{36}, \delta x_{4}=-x_{26}-x_{46}, \delta x_{5}=-x_{36}+x_{56},
$$

which implies $b_{1}(M)=1$.

One computes the differential of the non-exact generators of degree two in the Chevalley-Eilenberg complex as

$$
\begin{array}{lll}
\delta x_{12}=x_{126}, & \delta x_{13}=-x_{136}, & \delta x_{14}=x_{126}+x_{146}-x_{234}, \\
\delta x_{15}=x_{136}-x_{156}-x_{235}, & \delta x_{16}=x_{236}, & \delta x_{24}=2 x_{246} \\
\delta x_{25}=x_{236}, & \delta x_{34}=-x_{236}, & \delta x_{35}=-2 x_{356} \\
\delta x_{45}=x_{256}-x_{346}, & &
\end{array}
$$

i.e. $b_{2}(M)=2$.

The minimal model $\rho:(\bigwedge V, d) \rightarrow\left(\bigwedge \mathfrak{g}^{*}, \delta\right)$ must contain three closed generators $y, z_{1}, z_{2}$ which map to $x_{6}, x_{16}+x_{25}$ and $x_{16}-x_{34} . \quad \rho\left(y z_{1}\right)=x_{256}$ and $\rho\left(y z_{2}\right)=-x_{346}$ are closed and not exact. But in the generation of $y, z_{1}$ and $z_{2}$ is one (and up to a scalar only one) element that maps onto an exact form, namely $\rho\left(y\left(z_{1}+z_{2}\right)\right)=\delta x_{45}$. The minimal model's construction in the proof of Theorem 1.1.2 implies that there is another generator $u$ of degree two such that $\rho(u)=x_{45}$ 
and $d u=y\left(z_{1}+z_{2}\right)$. Since $\rho(y u)=x_{456}$ is closed and non-exact, there are no further generators of degree less than or equal to two in $V$. But this implies for each closed element $c$ of degree two that $y(u+c)$ is closed and non-exact in $\mathcal{M}$. Using the notation of Theorem 1.1.5, we have $u \in N^{2}, y \in V^{1}$ and $\mathcal{M}$ is not formal.

Finally, the existence of a symplectic form on $G / \Gamma$ follows from Proposition 8.3.1.

\section{Proposition 8.3.3.}

(i) Each quotient of the Lie group $G_{6.38}^{0}$ by a lattice is symplectic. $G_{6.38}^{0}$ contains a lattice $\Gamma$ with $b_{1}\left(G_{6.38}^{0} / \Gamma\right)=1$.

(ii) If the Lie group $G_{6.38}^{0}$ contains a lattice $\Gamma$ such that $M:=G_{6.38}^{0} / \Gamma$ satisfies $b_{1}(M)=1$ and $b_{2}(M)=2$, then $M$ is a symplectic and non-formal manifold.

Proof. The proof is similar to that of the last theorem. Therefore, we just give a sketch of the proof.

ad (i): The existence of a symplectic form on each quotient of $G:=G_{6.38}^{0}$ by a lattice follows from Proposition 8.3.1.

The nilradical $N$ of $G$ is the same as in the proof of Theorem $[8.3 .2$, so we have $[N, N]=\mathbb{R}$ and $\bar{N}=N /[N, N]=\mathbb{R}^{4}$. If $\bar{\mu}(t): \bar{N} \rightarrow \bar{N}$ is defined by

$$
\begin{aligned}
\bar{\mu}(t)((y, z, v, w)) & =\exp ^{G L(4, \mathbb{R})}\left(t\left(\begin{array}{cccc}
0 & 1 & 0 & 0 \\
-1 & 0 & 0 & 0 \\
-1 & 0 & 0 & 1 \\
0 & -1 & -1 & 0
\end{array}\right)\right)\left(\begin{array}{c}
y \\
z \\
v \\
w
\end{array}\right) \\
& =\left(\begin{array}{cccc}
\cos (t) & \sin (t) & 0 & 0 \\
-\sin (t) & \cos (t) & 0 & 0 \\
-t \cos (t) & -t \sin (t) & \cos (t) & \sin (t) \\
t \sin (t) & -t \cos (t) & -\sin (t) & \cos (t)
\end{array}\right)\left(\begin{array}{c}
y \\
z \\
v \\
w
\end{array}\right),
\end{aligned}
$$

one calculates that $\mu: \mathbb{R} \rightarrow \mathrm{A}(N)$ given by

$$
\begin{aligned}
\mu(t)((x, y, z, v, w))= & \left(x-\sin ^{2}(t) y z+\frac{\sin (t) \cos (t)}{2}\left(z^{2}-y^{2}\right)+t \frac{\sqrt{3}}{8}(y-z),\right. \\
& \bar{\mu}(t)((y, z, v, w)))
\end{aligned}
$$

is a one-parameter group with $d_{e}(\mu(t))=\exp ^{A(\mathfrak{n})}(t \underbrace{\left(\begin{array}{ccccc}0 & 0 & 0 & 0 & 0 \\ 0 & -1 & 0 & 0 & 0 \\ 0 & 0 & 1 & 0 & 0 \\ 0 & -1 & 0 & -1 & 0 \\ 0 & 0 & -1 & 0 & 1\end{array}\right)}_{=\operatorname{ad}\left(X_{6}\right)})$,

i.e. $G=N \ltimes_{\mu} \mathbb{R}$. (Here $X_{6}$ is chosen as in the last line of Table 21 on page 89.) 
For $t_{1}:=\frac{\pi}{3}$ we have

$$
\mu\left(t_{1}\right)((x, y, z, v, w))=\left(x-\frac{3}{4} y z+\frac{\sqrt{3}}{8}\left(z^{2}-y^{2}\right)+\frac{\pi}{8 \sqrt{3}}(y-z), \bar{\mu}(t)((y, z, v, w))\right),
$$

and in order to construct a lattice in $G$, it is enough to construct a lattice in $N$ that is preserved by $\mu\left(t_{1}\right) . \bar{\mu}\left(t_{1}\right)$ is conjugate to $A:=\left(\begin{array}{cccc}-1 & -3 & 0 & 0 \\ 1 & 2 & 0 & 0 \\ -2 & -3 & -1 & -3 \\ 1 & 1 & 1 & 2\end{array}\right)$ and the transformation matrix $T \in \mathrm{GL}(4, \mathbb{R})$ with $T A T^{-1}=\bar{\mu}\left(t_{1}\right)$ is

$$
T=\left(\begin{array}{cccc}
\frac{\sqrt{3}}{\pi} & 0 & 0 & 0 \\
-\frac{3}{\pi} & -\frac{6}{\pi} & 0 & 0 \\
0 & 0 & -\frac{2}{\sqrt{3}} & -\sqrt{3} \\
0 & 0 & 0 & 1
\end{array}\right)
$$

Denote by $\left\{b_{1}, \ldots, b_{4}\right\}$ the basis of $\mathbb{R}^{4}$ for which $\bar{\mu}\left(t_{1}\right)$ is represented by $A$, i.e. $b_{i}$ is the $i$-th column of $T$. One calculates

$$
\begin{aligned}
b_{11} b_{22}-b_{12} b_{21} & =\frac{-6 \sqrt{3}}{\pi^{2}} \\
b_{i 1} b_{j 2}-b_{i 2} b_{j 1} & =0 \text { for } i<j,(i, j) \neq(1,2) .
\end{aligned}
$$

This implies that we have for $\gamma_{0}:=\left(b_{11} b_{22}-b_{12} b_{21}, 0_{\mathbb{R}^{4}}\right), \gamma_{i}:=\left(b_{i 0}, b_{i}\right) \in N$ with arbitrary $b_{i 0} \in \mathbb{R}, i=1, \ldots, 4$,

$$
\left[\gamma_{1}, \gamma_{2}\right]=\gamma_{0},\left[\gamma_{1}, \gamma_{3}\right]=\left[\gamma_{1}, \gamma_{4}\right]=\left[\gamma_{2}, \gamma_{3}\right]=\left[\gamma_{2}, \gamma_{4}\right]=\left[\gamma_{3}, \gamma_{4}\right]=e_{N}
$$

If we set $b_{10}=\frac{1488 \sqrt{3}+72 \sqrt{3} \pi-19 \sqrt{3} \pi^{2}+4 \pi^{3}}{128 \pi^{2}}, b_{20}=\frac{2736 \sqrt{3}+216 \sqrt{3} \pi-25 \sqrt{3} \pi^{2}+12 \pi^{3}}{128 \pi^{2}}$ and $b_{30}=b_{40}=0$, we obtain

$$
\begin{array}{lllllll}
\mu\left(t_{1}\right)\left(\gamma_{0}\right) & = & \gamma_{0}, & & & & \\
\mu\left(t_{1}\right)\left(\gamma_{1}\right) & = & & \gamma_{1}^{-1} & \gamma_{2} & \gamma_{3}^{-2} & \gamma_{4}, \\
\mu\left(t_{1}\right)\left(\gamma_{2}\right) & = & & \gamma_{1}^{-3} & \gamma_{2}^{2} & \gamma_{3}^{-3} & \gamma_{4}, \\
\mu\left(t_{1}\right)\left(\gamma_{3}\right) & = & & & & \gamma_{3}^{-1} & \gamma_{4}, \\
\mu\left(t_{1}\right)\left(\gamma_{4}\right) & = & & & & \gamma_{3}^{-3} & \gamma_{4}^{2} .
\end{array}
$$

Then $\left\langle\exp ^{N}\left(\operatorname{Span}_{\mathbb{Z}} \log ^{N}\left(\left\{\gamma_{0}, \ldots, \gamma_{4}\right\}\right)\right)\right\rangle$ is a lattice in $N$. This can be seen by a similar computation as in the proof of the last theorem. Finally, one checks that the abelianisation of this lattice is isomorphic to $\mathbb{Z}$, hence the corresponding solvmanifold has $b_{1}=1$.

ad (ii): Let $\Gamma$ be a lattice in $G$ such that $b_{1}(G / \Gamma)=1$ and $b_{2}(G / \Gamma)=2$.

The Chevalley-Eilenberg complex $\left(\bigwedge \mathfrak{g}^{*}, \delta\right)$ has the closed generator $x_{6}$ and $\delta$ is given on the non-closed generators by

$$
\delta x_{1}=-x_{23}, \delta x_{2}=x_{36}, \delta x_{3}=-x_{26}, \delta x_{4}=-x_{26}+x_{56}, \delta x_{5}=-x_{36}-x_{46},
$$


which implies $H^{1}\left(\bigwedge \mathfrak{g}^{*}, \delta\right)=\left\langle\left[x_{6}\right]\right\rangle$.

One computes the differential of the non-exact generators of degree two in the Chevalley-Eilenberg complex as

$$
\begin{array}{llrl}
\delta x_{12}=-x_{136}, & \delta x_{13}=x_{126}, \\
\delta x_{14}=x_{126}-x_{156}-x_{234}, & \delta x_{15}=x_{136}+x_{146}-x_{235}, \\
\delta x_{16}=-x_{236}, & \delta x_{24}=-x_{256}-x_{346}, \\
\delta x_{25}=x_{236}+x_{246}-x_{356}, & \delta x_{34}=-x_{236}+x_{246}-x_{356}, \\
\delta x_{35}=x_{256}+x_{346}, & \delta x_{45}=x_{256}-x_{346},
\end{array}
$$

i.e. $H^{2}\left(\bigwedge \mathfrak{g}^{*}, \delta\right)=\left\langle\left[x_{16}+\frac{1}{2} x_{25}-\frac{1}{2} x_{34}\right],\left[x_{24}+x_{35}\right]\right\rangle$.

This implies that $G / \Gamma$ and $\left(\bigwedge \mathfrak{g}^{*}, \delta\right)$ have the same Betti numbers and therefore, by Theorem 3.11, they share their minimal model.

The minimal model $\rho:(\bigwedge V, d) \rightarrow\left(\bigwedge \mathfrak{g}^{*}, \delta\right)$ must contain three closed generators $y, z_{1}, z_{2}$ which map to $x_{6}, x_{16}+\frac{1}{2} x_{25}-\frac{1}{2} x_{34}$ and $x_{24}+x_{35} . \rho\left(y z_{2}\right)=x_{246}+x_{356}$ is closed and not exact. But $\rho\left(y z_{1}\right)=\frac{1}{2}\left(x_{256}-x_{346}\right)=\frac{1}{2} \delta x_{45}$ is exact. Hence the minimal model's construction in the proof of Theorem 1.1.2 implies that there is another generator $u$ of degree two such that $\rho(u)=\frac{1}{2} x_{45}$ and $d u=y z_{1}$. Since $\rho(y u)=\frac{1}{2} x_{456}$ is closed and non-exact, there are no further generators of degree less than or equal to two in $V$. Using the notation of Theorem 1.1.5, we have $u \in N^{2}, y \in V^{1},(u+c) y$ is closed and not exact for each $c \in C^{2}$ and $(\bigwedge V, d)$ is not formal.

\section{Theorem 8.3.4.}

(i) The completely solvable Lie group $G:=G_{6.78}$ possesses a lattice.

(ii) For each lattice the corresponding quotient is a symplectic and formal manifold with $b_{1}=b_{2}=1$.

Proof. ad (i): By definition, we have $G=\mathbb{R} \ltimes_{\mu} N$ with $N=G_{5.3}$ and $\mu(t)=\exp ^{N} \circ \exp ^{A(\mathfrak{n})}\left(t \operatorname{ad}\left(X_{6}\right)\right) \circ \log ^{N}$, where $\left\{X_{1}, \ldots, X_{6}\right\}$ denotes a basis of $\mathfrak{g}$ as in the second row of Table 27. Note that $\left\{X_{1}, \ldots, X_{5}\right\}$ is a basis for the nilradical $\mathfrak{n}$. One computes

$$
\mu(t)_{*}:=d_{e}(\mu(t))=\exp ^{A(\mathfrak{n})}\left(t \operatorname{ad}\left(X_{6}\right)\right)=\left(\begin{array}{ccccc}
e^{t} & 0 & 0 & 0 & 0 \\
0 & 1 & 0 & 0 & 0 \\
0 & 0 & e^{-t} & -t e^{-t} & 0 \\
0 & 0 & 0 & e^{-t} & 0 \\
0 & 0 & 0 & 0 & e^{t}
\end{array}\right) .
$$

Using

$$
\mathfrak{n}=\left\langle X_{5}\right\rangle \ltimes_{a d\left(X_{5}\right)}\left(\left\langle X_{1}\right\rangle \oplus\left\langle X_{2}, X_{3}, X_{4} \mid\left[X_{2}, X_{4}\right]=X_{3}\right\rangle\right)
$$


with $\operatorname{ad}\left(X_{5}\right)\left(X_{2}\right)=-X_{1}, \operatorname{ad}\left(X_{5}\right)\left(X_{4}\right)=-X_{2}, \operatorname{ad}\left(X_{5}\right)\left(X_{1}\right)=\operatorname{ad}\left(X_{5}\right)\left(X_{3}\right)=0$ and

$$
\left\langle X_{2}, X_{3}, X_{4} \mid\left[X_{2}, X_{4}\right]=X_{3}\right\rangle \cong \mathfrak{g}_{3.1},
$$

we can determine the Lie group $N$.

As a smooth manifold $N$ equals $\mathbb{R}^{5}$, and the multiplication is given by

$$
\begin{aligned}
& (a, b, c, r, s) \cdot(x, y, z, v, w) \\
& \quad=\left(a+x+b w+\frac{r w^{2}}{2}, b+y+r w, c+z+b v+\frac{r^{2} w}{2}+r v w, r+v, s+w\right) .
\end{aligned}
$$

Now, Theorem 4.2 enables us to compute the exponential map of $N$ as

$$
\begin{aligned}
& \exp ^{N}\left(x X_{1}+y X_{2}+z X_{3}+v X_{4}+w X_{5}\right) \\
& =\left(x+\frac{y w}{2}+\frac{v w^{2}}{6}, y+\frac{v w}{2}, z+\frac{y v}{2}+\frac{v^{2} w}{3}, v, w\right),
\end{aligned}
$$

and therefore, we also obtain the logarithm of $N$

$$
\begin{aligned}
& \log ^{N}((x, y, z, v, w)) \\
& \quad=\left(x-\frac{y w}{2}+\frac{v w^{2}}{12}\right) X_{1}+\left(y-\frac{v w}{2}\right) X_{2}+\left(z-\frac{y v}{2}-\frac{v^{2} w}{12}\right) X_{3}+v X_{4}+w X_{5} .
\end{aligned}
$$

Finally, a short computation shows that (12) implies

$$
\mu(t)((x, y, z, v, w))=\left(e^{t} x, y, e^{-t}(z-t w), e^{-t} v, e^{t} w\right) .
$$

Let $t_{1}:=\ln \left(\frac{3+\sqrt{5}}{2}\right), b_{0}:=-\frac{2 t_{1}}{1+\sqrt{5}}$ and consider for $t \in \mathbb{R}$ the automorphisms $I(t): N \rightarrow N$ given by

$$
\begin{aligned}
& I(t)((x, y, z, v, w)) \\
& \quad=\left(0, t b_{0}, 0,0,0\right)(x, y, z, v, w)\left(0, t b_{0}, 0,0,0\right)^{-1}=\left(x+t b_{0} w, y, z+t b_{0} v, v, w\right),
\end{aligned}
$$

and $\nu(t):=\mu(t) \circ I(t): N \rightarrow N$. It is easy to see that $\nu: \mathbb{R} \rightarrow \mathrm{A}(N)$ is a one-parameter group in $N$.

We shall show that there exists a lattice $\Gamma_{N}$ in $N$ preserved by $\nu\left(t_{1}\right)$, and this then implies the existence of a lattice in $G_{6.78}$, namely $t_{1} \mathbb{Z} \ltimes_{\nu} \Gamma_{N}$.

For the remainder of the proof, we identify $\mathfrak{n} \equiv \mathbb{R}^{5}$ with respect to the basis $\left\{X_{1}, \ldots, X_{5}\right\}$ of $\mathfrak{n}$. Under this identification, consider the basis $\left\{Y_{1}, \ldots, Y_{5}\right\}$ of $\mathfrak{n}, Y_{i}$ being the $i$-th column of $T=\left(T_{i j}\right) \in \mathrm{GL}(5, \mathbb{R})$, where $T$ has the following entries:

$$
\begin{aligned}
& T_{11}=\frac{10(161+72 \sqrt{5}) \ln \left(\frac{3+\sqrt{5}}{2}\right)^{2}}{1165+521 \sqrt{5}} \\
& T_{12}=0
\end{aligned}
$$




$$
\begin{aligned}
& T_{13}=\frac{5(2+\sqrt{5})(161+72 \sqrt{5}) \ln \left(\frac{3+\sqrt{5}}{2}\right)^{2}}{1525+682 \sqrt{5}} \\
& T_{14}=\frac{328380+146856 \sqrt{5}-(159975+71543 \sqrt{5}) \ln \left(\frac{3+\sqrt{5}}{2}\right)^{2}}{202950+90762 \sqrt{5}} \\
& T_{15}=1 \\
& T_{21}=0 \\
& T_{22}=-\frac{(5+3 \sqrt{5}) \ln \left(\frac{3+\sqrt{5}}{2}\right)}{3+\sqrt{5}}, \\
& T_{23}=0, \\
& T_{24}=-\frac{(158114965+70711162 \sqrt{5}) \ln \left(\frac{3+\sqrt{5}}{2}\right)}{141422324+63245986 \sqrt{5}}, \\
& T_{25}=\frac{5(3940598+1762585 \sqrt{5}) \ln \left(\frac{3+\sqrt{5}}{2}\right)}{17622890+7881196 \sqrt{5}}, \\
& T_{31}=\frac{1}{2}(5+\sqrt{5}) \ln \left(\frac{3+\sqrt{5}}{2}\right), \\
& T_{32}=0 \\
& T_{33}=T_{22}, \\
& T_{34}=1 \\
& T_{35}=-\frac{597+267 \sqrt{5}+(3808+1703 \sqrt{5}) \ln \left(\frac{3+\sqrt{5}}{2}\right)}{369+165 \sqrt{5}} \\
& T_{41}=0 \\
& T_{42}=0 \\
& T_{43}=0 \\
& T_{44}=1 \\
& T_{45}=-\frac{2(2+\sqrt{5})}{3+\sqrt{5}}, \\
& T_{51}=0 \\
& T_{52}=0 \\
& T_{53}=0 \\
& T_{54}=\ln \left(\frac{2}{3+\sqrt{5})},\right. \\
& T_{55}=-\frac{2 \ln \left(\frac{3+\sqrt{5}}{2}\right)}{1+\sqrt{5}} . \\
& = \\
& =
\end{aligned}
$$


Let $\gamma_{i}:=\exp ^{N}\left(Y_{i}\right)$ for $i \in\{1, \ldots 5\}$ and

$$
\begin{aligned}
S_{1}= & \frac{92880525355200+41537433696024 \sqrt{5}}{57403321562460+25671545829588 \sqrt{5}} \\
& -\frac{(3591421616495+1606132574069 \sqrt{5}) \ln \left(\frac{3+\sqrt{5}}{2}\right)^{2}}{57403321562460+25671545829588 \sqrt{5}}, \\
S_{2}= & -\frac{(228826127+102334155 \sqrt{5}) \ln \left(\frac{3+\sqrt{5}}{2}\right)}{141422324+63245986 \sqrt{5}}, \\
S_{3}= & -\frac{(757189543+338625458 \sqrt{5}) \ln \left(\frac{3+\sqrt{5}}{2}\right)}{848533944+379475916 \sqrt{5}}, \\
S_{4}= & \frac{724734510+324111126 \sqrt{5}-(325041375+145362922 \sqrt{5}) \ln \left(\frac{3+\sqrt{5}}{2}\right)^{2}}{724734510+324111126 \sqrt{5}}, \\
S_{5}= & \frac{(120789085+54018521 \sqrt{5}) \ln \left(\frac{3+\sqrt{5}}{2}\right)}{74651760+33385282 \sqrt{5}}, \\
S_{6}= & -\frac{466724522940+208725552012 \sqrt{5}}{24(12018817440+5374978561 \sqrt{5})} \\
& +\frac{(3393446021605+1517595196457 \sqrt{5}) \ln \left(\frac{3+\sqrt{5}}{2}\right)}{24(12018817440+5374978561 \sqrt{5})} .
\end{aligned}
$$

One computes $\gamma_{1}=\left(T_{11}, 0, T_{31}, 0,0\right), \gamma_{2}=\left(0, T_{22}, 0,0,0\right), \gamma_{3}=\left(T_{13}, 0, T_{33}, 0,0\right)$, $\gamma_{4}=\left(S_{1}, S_{2}, S_{3}, T_{44}, T_{54}\right)$ and $\gamma_{5}=\left(S_{4}, S_{5}, S_{6}, T_{45}, T_{55}\right)$.

Moreover, if $A$ denotes the matrix $\left(\begin{array}{ccccc}1 & 0 & 1 & \frac{13}{6} & \frac{11}{6} \\ 0 & 1 & 0 & 0 & -\frac{1}{2} \\ 1 & 0 & 2 & -\frac{5}{6} & -\frac{1}{3} \\ 0 & 0 & 0 & 2 & 1 \\ 0 & 0 & 0 & 1 & 1\end{array}\right)$, we can calculate $T A T^{-1}=\nu\left(t_{1}\right)_{*}:=d_{e}(\nu(t))$. Since $\nu\left(t_{1}\right)=\exp ^{N} \circ \nu\left(t_{1}\right)_{*} \circ \log ^{N}$, this yields $\nu\left(t_{1}\right)\left(\gamma_{1}\right)=\gamma_{1} \gamma_{3}, \nu\left(t_{1}\right)\left(\gamma_{2}\right)=\gamma_{2}, \nu\left(t_{1}\right)\left(\gamma_{3}\right)=\gamma_{1} \gamma_{3}^{2}, \nu\left(t_{1}\right)\left(\gamma_{4}\right)=\gamma_{1}^{2} \gamma_{2}^{-2} \gamma_{4}^{2} \gamma_{5}$ and $\nu\left(t_{1}\right)\left(\gamma_{5}\right)=\gamma_{1}^{2} \gamma_{2}^{-1} \gamma_{4} \gamma_{5}$.

Therefore, we have shown that $\nu\left(t_{1}\right)$ preserves the subgroup $\Gamma_{N}$ of $N$ which is generated by $\gamma_{1}, \ldots, \gamma_{5}$. In order to complete the proof of (i), it suffices to show that $\Gamma_{N}$ is a lattice in $N$.

Since $\mathfrak{n}$ is 3-step nilpotent, the Baker-Campbell-Hausdorff formula (see e.g. [59, Chapter 2.15]) yields for all $V, W \in \mathfrak{n}$

$$
\log ^{N}\left(\exp ^{N}(V) \exp ^{N}(W)\right)=V+W+\frac{1}{2}[V, W]+\frac{1}{12}([[V, W], W]-[[V, W], V]) .
$$

Therefore, we obtain by a short calculation $\left[Y_{2}, Y_{4}\right]=Y_{3},\left[Y_{2}, Y_{5}\right]=Y_{1}$ and $\left[Y_{4}, Y_{5}\right]=\frac{1}{2} Y_{1}+Y_{2}+\frac{1}{2} Y_{3}$, i.e. the basis $\left\{Y_{1}, \ldots, Y_{5}\right\}$ has rational structure constants. Theorem 2.1 then implies that $\Gamma_{N}$ is a lattice in $N$. 
ad (ii): Let $\Gamma$ be a lattice in $G:=G_{6.78}$. By completely solvability and Theorem 3.11 (ii), the minimal model of $M=G / \Gamma$ is the same as the minimal model $\mathcal{M}$ of the Chevalley-Eilenberg complex $\left(\bigwedge \mathfrak{g}^{*}, \delta\right)$. In view of Theorem 1.1.6, it suffices to prove that the latter is 2 -formal. On the non-closed generators of $\left(\bigwedge \mathfrak{g}^{*}, \delta\right)$ the differential is given by

$$
\delta x_{1}=x_{16}-x_{25}, \delta x_{2}=-x_{45}, \delta x_{3}=-x_{24}-x_{36}-x_{46}, \delta x_{4}=-x_{46}, \delta x_{5}=x_{56},
$$

i.e. $H^{1}\left(\bigwedge \mathfrak{g}^{*}, \delta\right)=\left\langle\left[x_{6}\right]\right\rangle$. Further, one calculates $H^{2}\left(\bigwedge \mathfrak{g}^{*}, \delta\right)=\left\langle x_{14}+x_{26}+x_{35}\right\rangle$. The minimal model $\rho:(\bigwedge V, d) \rightarrow\left(\bigwedge \mathfrak{g}^{*}, \delta\right)$ then must contain two closed generators $y, z$ which map to $x_{6}$ and $x_{14}+x_{26}+x_{35}$. Since $\rho(y z)=x_{146}+x_{356}$ is closed and non-exact, there are no other generators of degree two in $(\bigwedge V, d)$, hence up to degree two, all generators are closed. This implies the minimal model's 2-formality.

Moreover, $x_{14}+x_{26}+x_{35}$ defines a symplectic form.

Remark. In order to determine a lattice in $G_{6.78}$, the author also found a lattice of the completely solvable Lie group $G_{6.76}^{-1}$. One can show that the corresponding solvmanifold is formal and has first Betti number equal to one. Unfortunately, it is not symplectic by Proposition 8.3.1.

Remark. Besides the mentioned groups above, the following non-completely solvable Lie groups $G_{6 . i}$ could give rise to a symplectic solvmanifold $G_{6 . i} / \Gamma$ with $b_{1}\left(G_{6 . i} / \Gamma\right)=1$ :

$$
\begin{array}{lll}
i=8 ; & i=9, \quad b \neq 0 ; & i=10, \quad a \neq 0 ; \\
i=11 ; & i=12 ; & i=32, \quad a=\varepsilon=0<h ; \\
i=37, \quad a=0 ; & i=88, \mu_{0} \nu_{0} \neq 0 ; \quad i=89, \nu_{0} s \neq 0 ; \\
i=90, \quad \nu_{0} \neq 0 ; & i=92, \quad \mu_{0} \nu_{0} \neq 0 ; \quad i=92^{*} ; \\
i=93, \quad\left|\nu_{0}\right|>\frac{1}{2} . & &
\end{array}
$$

But then the cohomology class of the symplectic form cannot lie in the image of the inclusion $H^{*}\left(\bigwedge \mathfrak{g}_{6 . i}^{*}, \delta\right) \hookrightarrow H^{*}\left(G_{6 . i} / \Gamma, d\right)$ by Proposition 8.3.1.

\subsection{Symplectic solvmanifolds whose first Betti number is greater than one}

In this section, we examine which Lie groups $G$ can give rise to a six-dimensional solvmanifold $G / \Gamma$ with $b_{1}(G / \Gamma)>1$. Again, we just consider indecomposable connected and simply-connected solvable Lie groups. The nilradical of such a group has not dimension equal to three, see e.g. [43]. Proposition 3.5 then tells us that indecomposable solvable Lie groups have nilradicals of dimension greater than three. Moreover, the nilpotent ones were considered in Section 8.1, hence we can assume that $G$ is non-nilpotent, i.e. $\operatorname{dim} \operatorname{Nil}(G) \in\{4,5\}$. The corresponding Lie algebras are listed in Tables $17-33$. 
In Section 8.2, we have excluded some groups $G$ since they cannot admit lattices. Clearly, we omit them in the considerations below.

By Theorem 3.11(ii), we have in the completely solvable case an isomorphism from Lie algebra cohomology to the solvmanifold's cohomology, i.e. the Lie algebra $\mathfrak{g}$ must satisfy $b_{1}(\mathfrak{g})>1$, too. In the last section, we saw that $\mathfrak{g}_{6.38}^{0}$ is the only non-completely solvable but cohomologically symplectic Lie algebra with $b_{1}(\mathfrak{g})=1$. Therefore, for each lattice $\Gamma$ in $G_{6.38}^{0}$ with $b_{1}\left(G_{6.38}^{0} / \Gamma\right)>1$, the quotient is symplectic. We now turn to Lie algebras with $b_{1}(\mathfrak{g})>1$. The possible values of $b_{1}$ can be read of in Tables 34- 36.

The remaining algebras to examine are $\mathfrak{g}_{6 . i}$ with

$$
\begin{array}{lll}
i=2, a=0 ; & i=3, d=-1 ; & i=6, a=-\frac{1}{2}, b=0 ; \\
i=9, b=0 ; & i=10, a=0 ; & i=21, a=0 ; \\
i=23, a=0 ; & i=25, b=0 ; & i=26 ; \\
i=29, b=0 ; & i=33, a=0 ; & i=34, a=0 ; \\
i=36, a=0 ; & i=54, l=-1 ; & i=63 ; \\
i=65, l=0 ; & i=70, p=0 ; & i=83, l=0 ; \\
i=84 ; & i=88, \mu_{0}=\nu_{0}=0 ; & i=89, \nu_{0} s=0 ; \\
i=90, \nu_{0}=0 ; & i=92, \nu_{0} \mu_{0}=0 ; & i=93, \nu_{0}=0 ; \\
i=102 ; & i=105 ; & i=107 ; \\
i=113 ; & i=114 ; & i=115 ; \\
i=116 ; & i=118 ; & i=120 ; \\
i=125 ; & i=129 ; & i=135 .
\end{array}
$$

As above, we just consider such Lie algebras that are cohomologically symplectic, although this condition is only in the completely solvable case necessary for the existence of a symplectic form on $G / \Gamma$.

Proposition 8.4.1. Let $\mathfrak{g}_{6 . i}$ be one of the Lie algebras listed in (14).

Then $\mathfrak{g}_{6 . i}$ is cohomologically symplectic if and only if it is contained in the following list:

$$
\begin{array}{rlrl}
b_{1}=2: & i=3 \wedge d=-1, & i=10 \wedge a=0, \quad i=21 \wedge a=0, \\
& i=36 \wedge a=0, & i=54 \wedge l=-1, \\
& i=70 \wedge p=0, & i=118 \wedge b= \pm 1 . & \\
b_{1}=3: & i=23 \wedge a=0 \wedge \varepsilon \neq 0, & i=29 \wedge b=0 .
\end{array}
$$

Proof. This is done by a case by case analysis as described in the proof of Proposition 8.3.1. We list the symplectic forms for the Lie algebras that are cohomologically symplectic. In the cases with $b_{1}=2$, the symplectic forms are 
given by

$$
\begin{array}{lll}
i=3, d=-1 & : \quad \omega=\lambda x_{16}+\mu x_{23}+\nu x_{45}, & \lambda \mu \nu \neq 0, \\
i=10, a=0 \quad: \quad \omega=\lambda x_{16}+\mu x_{23}+\nu x_{45}, & \lambda \mu \nu \neq 0, \\
i=21, a=0 \quad: \quad \omega=\lambda x_{12}+\mu x_{36}+\nu x_{45}, & \lambda \mu \nu \neq 0, \\
i=36, a=0: \quad \omega=\lambda x_{12}+\mu x_{36}+\nu x_{45}, & \lambda \mu \nu \neq 0, \\
i=54, l=-1: & \omega=\lambda\left(x_{12}+x_{23}\right)+\mu x_{34}+\nu x_{56}, & \lambda \nu \neq 0, \\
i=70, p=0: & \omega=\lambda\left(x_{13}+x_{24}\right)+\mu x_{34}+\nu x_{56}, & \lambda \nu \neq 0, \\
i=118, b= \pm 1: & \omega=\lambda\left(x_{13} \pm x_{24}\right)+\mu\left(x_{14}-x_{23}\right)+\nu x_{56}, & \left(\lambda^{2}+\mu^{2}\right) \nu \neq 0 .
\end{array}
$$

In the cases with $b_{1}=3$, we have the symplectic forms

$$
\omega=\lambda\left(x_{12}+\varepsilon x_{35}\right)+\mu\left(x_{16}+x_{24}\right)+\nu\left(x_{23}-\varepsilon x_{56}\right)+\rho x_{25}+\sigma x_{46}
$$

with $\lambda \mu \nu \neq 0$ for $i=23, a=0, \varepsilon \neq 0$,

$$
\omega=\lambda\left(x_{13}+\varepsilon x_{45}\right)+\mu\left(x_{16}+x_{24}\right)+\nu\left(x_{23}-\varepsilon x_{56}\right)+\rho x_{26}+\sigma x_{34}
$$

with $\lambda \neq 0, \rho \neq \frac{(\lambda+\varepsilon) \mu \nu}{\lambda}$ for $i=29, b=0, \varepsilon \neq 0$ and

$$
\omega=\lambda x_{12}+\mu x_{13}+\nu\left(x_{16}+x_{24}\right)+\rho x_{26}+\sigma x_{34}+\tau x_{56}
$$

with $\nu(\nu \sigma+\mu \tau) \neq 0$ for $i=29, b=0, \varepsilon \neq 0$.

Provided there is a lattice in one of the ten Lie groups $G_{6 . i}$ in the last proposition whose Lie algebras are cohomologically symplectic, we can ensure that the corresponding solvmanifold is symplectic. In the completely solvable case, i.e. $i \in\{3,21,23,29,54\}$, we can determine cohomological properties of the potential solvmanifolds.

\section{Proposition 8.4.2.}

(i) There is a lattice in the completely solvable Lie group $G_{6.3}^{0,-1}$.

(ii) For each lattice the corresponding solvmanifold is symplectic, not formal and satisfies $b_{1}=2$ as well as $b_{2}=3$.

Proof. ad (i) : We have $G:=G_{6.3}^{0,-1}=\mathbb{R} \ltimes_{\mu} \mathbb{R}^{4}$ with $\mu(t)=\exp ^{G L(4, \mathbb{R})}\left(t \operatorname{ad}\left(X_{6}\right)\right)$, where $X_{6} \in \mathfrak{g}_{6.3}^{0,-1}$ is chosen as in Table 18 , i.e.

$$
\mu(t)=\left(\begin{array}{ccccc}
1 & -t & \frac{t^{2}}{2} & 0 & 0 \\
0 & 1 & -t & 0 & 0 \\
0 & 0 & 1 & 0 & 0 \\
0 & 0 & 0 & e^{-t} & 0 \\
0 & 0 & 0 & 0 & e^{t}
\end{array}\right)
$$


Set $t_{1}:=\ln \left(\frac{3+\sqrt{5}}{2}\right)$. Then $\mu\left(t_{1}\right)$ is conjugate to $\left(\begin{array}{ccccc}1 & 1 & 0 & 0 & 0 \\ 0 & 1 & 1 & 0 & 0 \\ 0 & 0 & 1 & 0 & 0 \\ 0 & 0 & 0 & 0 & -1 \\ 0 & 0 & 0 & 1 & 3\end{array}\right)$. This follows from (4) and the fact that the Jordan form of the upper left block of $\mu\left(t_{1}\right)$ is $\left(\begin{array}{lll}1 & 1 & 0 \\ 0 & 1 & 1 \\ 0 & 0 & 1\end{array}\right)$. Hence $G$ admits a lattice.

ad (ii): By completely solvability and Theorem 3.11 (ii), the solvmanifold's minimal model is the same as the minimal model of the Chevalley-Eilenberg complex $\left(\bigwedge\left(x_{1}, \ldots, x_{6}\right), \delta\right)$. In view of Theorem 1.1.6, it suffices to prove that the latter is not 2 -formal.

Using the knowledge of the Chevalley-Eilenberg complex, one can compute $\rho:\left(\bigwedge\left(y_{1}, \ldots, y_{4}, z\right), d\right) \rightarrow\left(\bigwedge\left(x_{1}, \ldots, x_{6}\right), \delta\right)$ as the minimal model up to generators of degree two, where

$$
\begin{gathered}
\rho\left(y_{1}\right)=x_{3}, \rho\left(y_{2}\right)=x_{6}, \rho\left(y_{3}\right)=-x_{2}, \rho\left(y_{4}\right)=-x_{1}, \rho(z)=x_{4} x_{5}, \\
d y_{1}=d y_{2}=0, d y_{3}=y_{1} y_{2}, d y_{4}=y_{2} y_{3}, d z=0 .
\end{gathered}
$$

This obviously implies the statement about the Betti numbers. Moreover, using the notation of Theorem 1.1.5, we have $C^{1}=\left\langle y_{1}, y_{2}\right\rangle, N^{1}=\left\langle y_{3}, y_{4}\right\rangle$, and $y_{1}\left(y_{3}+c\right)$ is closed but not exact for each $c \in C^{1}$. Hence the minimal model is not 1formal.

\section{Proposition 8.4.3.}

(i) There is a lattice in the completely solvable Lie group $G_{6.21}^{0}$.

(ii) For each lattice the corresponding solvmanifold is symplectic, not formal and satisfies $b_{1}=2$ as well as $b_{2}=3$.

Proof. The proof of (ii) is completely analogous to that of (ii) in the last proposition. But this time, the minimal model is given by

$$
\begin{gathered}
\rho\left(y_{1}\right)=x_{2}, \rho\left(y_{2}\right)=x_{6}, \rho\left(y_{3}\right)=-x_{3}, \rho\left(y_{4}\right)=x_{1}, \rho(z)=x_{4} x_{5}, \\
d y_{1}=d y_{2}=0, d y_{3}=y_{1} y_{2}, d y_{4}=y_{1} y_{3}, d z=0,
\end{gathered}
$$

and $y_{1}\left(y_{4}+c\right)$ is closed but non-exact for each closed $c$ of degree one.

ad (i): In order to prove the existence of a lattice, we use the same argumentation as in the proof of Theorem 8.3.2 (i). (Note that $G_{6.15}^{-1}$ and $G:=G_{6.21}^{0}$ share their nilradical $N$.) But of course, we now have a different initial data: 


$$
\begin{aligned}
& G=\mathbb{R} \ltimes_{\mu} N \text { with } \mu(t)=\exp ^{N} \circ \exp ^{A(\mathfrak{n})}\left(t\left(\begin{array}{ccccc}
0 & 0 & 0 & 0 & 0 \\
0 & 0 & 0 & 0 & 0 \\
0 & -1 & 0 & 0 & 0 \\
0 & 0 & 0 & -1 & 0 \\
0 & 0 & 0 & 0 & 1
\end{array}\right)\right) \circ \log ^{N} \text { and } \\
& \bar{\mu}(t)((y, z, v, w))=\exp ^{G L(4, \mathbb{R})}\left(t\left(\begin{array}{cccc}
0 & 0 & 0 & 0 \\
-t & 0 & 0 & 0 \\
0 & 0 & -t & 0 \\
0 & 0 & 0 & t
\end{array}\right)\right)\left(\begin{array}{c}
y \\
z \\
v \\
w
\end{array}\right) \\
& =\left(\begin{array}{cccc}
1 & 0 & 0 & 0 \\
-t & 1 & 0 & 0 \\
0 & 0 & e^{-t} & 0 \\
0 & 0 & 0 & e^{t}
\end{array}\right)\left(\begin{array}{c}
y \\
z \\
v \\
w
\end{array}\right)
\end{aligned}
$$

Arguing analogous as in (10), one obtains

$$
\mu(t)((x, y, z, v, w))=\left(x-\frac{t}{2} y^{2}, \bar{\mu}(t)((x, y, z, v, w))\right) .
$$

Let $t_{1}=\ln \left(\frac{3+\sqrt{5}}{2}\right), A:=\left(\begin{array}{cccc}1 & 1 & 0 & 0 \\ 0 & 1 & 0 & 0 \\ 0 & 0 & 0 & -1 \\ 0 & 0 & 1 & 3\end{array}\right)$ and $T=\left(\begin{array}{cccc}0 & -\frac{1}{t_{1}} & 0 & 0 \\ 1 & 0 & 0 & 0 \\ 0 & 0 & \frac{18+8 \sqrt{5}}{7+3 \sqrt{5}} & 1 \\ 0 & 0 & \frac{2}{3+\sqrt{5}} & 1\end{array}\right)$.

Then we have $T A T^{-1}=\bar{\mu}\left(t_{1}\right)$. Denote the $i$-th column of T by $b_{i}$. Analogous calculations as in loc. cit. imply the existence of a lattice generated by $\gamma_{0}:=\left(\frac{1}{t_{1}}, 0_{\mathbb{R}^{4}}\right)$ and $\gamma_{i}:=\left(b_{i 0}, b_{i}\right), i \in\{1, \ldots, 4\}$, where $b_{20} \in \mathbb{R}$ arbitrary and $b_{10}=-\frac{1}{2 t_{1}}$ as well as $b_{30}=b_{40}=0$.

\section{Proposition 8.4.4.}

(i) Let $\varepsilon= \pm 1$. There is a lattice in the completely solvable Lie group $G_{6.23}^{0,0, \varepsilon}$.

(ii) If there is a lattice in $G_{6.23}^{0,0, \varepsilon}, \varepsilon \neq 0$, then the corresponding solvmanifold is symplectic, non-formal and satisfies $b_{1}=3$ as well as $b_{2}=5$.

Proof. ad (i): $G_{6.23}^{0,0, \varepsilon}$ has the same nilradical $N$ as $G_{6.15}^{-1}$ and the latter is described at the beginning of the proof of Theorem 8.3.2.

By definition, $G_{6.23}^{0,0, \varepsilon}=\mathbb{R} \ltimes_{\mu} N$ with

$$
\mu(t)=\exp ^{N} \circ \exp ^{A(\mathfrak{n})}\left(t\left(\begin{array}{ccccc}
0 & 0 & 0 & 0 & -\varepsilon \\
0 & 0 & 0 & 0 & 0 \\
0 & -1 & 0 & 0 & 0 \\
0 & 0 & -1 & 0 & 0 \\
0 & 0 & 0 & 0 & 0
\end{array}\right)\right) \circ \log ^{N}
$$


The functions $\exp ^{N}, \log ^{N}$ also can be found in the proof of Theorem 8.3.2. Using their knowledge, we calculate

$$
\mu(t)((x, y, z, v, w))=\left(x-\frac{t}{2} y^{2}-t \varepsilon, y, z-t y, \frac{t^{2}}{2} y-t z+v, w\right) .
$$

If $\varepsilon= \pm 1$, then the map $\mu(2)$ preserves the lattice

$$
\{(x, y, z, v, w) \in N \mid x, y, z, v, w \in \mathbb{Z}\} \subset N .
$$

Therefore, $G_{6.23}^{0,0, \varepsilon}$ admits a lattice.

ad (ii): By completely solvability, the Betti numbers of the Chevalley-Eilenberg complex coincide with the solvmanifold's Betti numbers. A short calculation yields the first Betti numbers of the former as $b_{1}=3$ and $b_{2}=5$.

As above, the knowledge of the Chevalley-Eilenberg complex $\left(\bigwedge\left(x_{1}, \ldots, x_{6}\right), \delta\right)$ enables us to compute the first stage of the minimal model. It is given by $\rho:\left(\bigwedge\left(y_{1}, \ldots, y_{6}\right), d\right) \rightarrow\left(\bigwedge\left(x_{1}, \ldots, x_{6}\right), \delta\right)$ with

$$
\begin{gathered}
\rho\left(y_{1}\right)=x_{2}, \rho\left(y_{2}\right)=x_{5}, \rho\left(y_{3}\right)=x_{6}, \rho\left(y_{4}\right)=-x_{3}, \rho\left(x_{5}\right)=x_{1}, \rho\left(y_{6}\right)=-x_{4} \\
d y_{1}=d y_{2}=d y_{3}=0, d y_{4}=y_{1} y_{3}, d y_{5}=y_{1} y_{4}-\varepsilon y_{2} y_{3}, d y_{6}=y_{3} y_{4} .
\end{gathered}
$$

Since $y_{3}\left(y_{6}+c\right)$ is closed and non-exact for each closed $c$ of degree one, the minimal model is not 1 -formal.

\section{Proposition 8.4.5.}

(i) Let $\varepsilon \in\{0, \pm 1\}$. There is a lattice in the completely solvable Lie group $G_{6.29}^{0,0, \varepsilon}$.

(ii) If there is a lattice in $G_{6.29}^{0,0, \varepsilon}, \varepsilon \in \mathbb{R}$, then the corresponding solvmanifold is symplectic, non-formal and has $b_{1}=3$ as well as $b_{2}=\left\{\begin{array}{ll}5, & \text { if } \varepsilon \neq 0 \\ 6, & \text { if } \varepsilon=0\end{array}\right\}$.

Proof. The argumentation is analogous to the last proof, but this time we have

$$
\mu(t)((x, y, z, v, w))=\left(x-\frac{\varepsilon}{6} t^{3} z+\frac{\varepsilon}{2} t^{2} v-\varepsilon t w, y, z,-t z+v, \frac{1}{2} t^{2} z-t v+w\right) .
$$

(Note that there is no misprint. The maps $\exp ^{N} \circ \exp ^{A(\mathfrak{n})}\left(t \operatorname{ad}\left(X_{6}\right)\right) \circ \log ^{N}$ and $\exp ^{A(\mathfrak{n})}\left(t \operatorname{ad}\left(X_{6}\right)\right)$ have the same form.) For $\varepsilon \in\{0, \pm 1\}, \mu(6)$ preserves the integer lattice mentioned in the last proof. This implies (i).

In order to prove (ii), we consider the minimal model. Up to generators of degree one, it is given by

$$
\begin{gathered}
\rho\left(y_{1}\right)=x_{2}, \rho\left(y_{2}\right)=x_{3}, \rho\left(y_{3}\right)=x_{6}, \rho\left(y_{4}\right)=-x_{4}, \rho\left(x_{5}\right)=-x_{5}, \rho\left(y_{6}\right)=-x_{1} \\
d y_{1}=d y_{2}=d y_{3}=0, d y_{4}=y_{2} y_{3}, d y_{5}=y_{3} y_{4}, d y_{6}=y_{1} y_{2}+\varepsilon y_{3} y_{5}
\end{gathered}
$$


if $\varepsilon \neq 0$, and

$$
\begin{gathered}
\rho\left(y_{1}\right)=x_{2}, \rho\left(y_{2}\right)=x_{3}, \rho\left(y_{3}\right)=x_{6}, \rho\left(y_{4}\right)=-x_{1}, \rho\left(x_{5}\right)=-x_{4}, \rho\left(y_{6}\right)=-x_{5} \\
d y_{1}=d y_{2}=d y_{3}=0, d y_{4}=y_{1} y_{2}, d y_{5}=y_{2} y_{3}, d y_{6}=y_{3} y_{5}
\end{gathered}
$$

if $\varepsilon=0$. In both cases $y_{2}\left(y_{4}+c\right)$ is closed and non-exact for all closed $c$ of degree one.

The following result is due to Fernández, de Léon and Saralegui. Its proof can be found in [20, Section 3]. Note that the cohomological results are independent of the choice of the lattice, since the Lie group in the proposition is completely solvable.

Proposition 8.4.6. The completely solvable Lie group $G_{6.54}^{0,-1}$ admits a lattice. For each such, the corresponding solvmanifold is symplectic, non-formal and satisfies $b_{1}=2$ as well as $b_{2}=5$.

Summing up the results concerning completely solvable Lie groups that admit symplectic quotients, we obtain:

Theorem 8.4.7. All six-dimensional symplectic solvmanifolds that can be written as quotient of a non-nilpotent completely solvable indecomposable Lie group are contained in one of the last five propositions, Theorem 8.3.2 or Theorem 8.3.4.

To end this section, we consider the four cohomologically symplectic Lie algebras $\mathfrak{g}_{6 . i}$ of Proposition 8.4.1 that are not completely solvable, this means $i=10 \wedge a=0, i=36 \wedge a=0, i=70 \wedge p=0$ or $i=118 \wedge b= \pm 1$. Clearly, the existence of a lattice implies that the corresponding solvmanifold is symplectic. But in order to make a statement about cohomological properties, one needs an assumption about the first two Betti numbers to ensure the knowledge of the cohomology algebra.

\section{Proposition 8.4.8.}

(i) Each quotient of the Lie group $G:=G_{6.10}^{0,0}$ by a lattice is symplectic and $G$ admits a lattice $\Gamma$ with $b_{1}(G / \Gamma)=2$.

(ii) If there is a lattice in $G$ such that the corresponding solvmanifold satisfies $b_{1}=2$ and $b_{2}=3$, then it is symplectic and not formal.

Proof. We have $G=\mathbb{R} \ltimes_{\mu} \mathbb{R}^{4}$ with $\mu(t)=\exp ^{G L(4, \mathbb{R})}\left(t \operatorname{ad}\left(X_{6}\right)\right)$ and $X_{6} \in \mathfrak{g}_{6.10}^{0,0}$ chosen as in Table 18, i.e.

$$
\mu(t)=\left(\begin{array}{ccccc}
1 & -t & \frac{t^{2}}{2} & 0 & 0 \\
0 & 1 & -t & 0 & 0 \\
0 & 0 & 1 & 0 & 0 \\
0 & 0 & 0 & \cos (t) & -\sin (t) \\
0 & 0 & 0 & \sin (t) & \cos (t)
\end{array}\right)
$$


$\mu(\pi)$ is conjugate to $\left(\begin{array}{ccccc}1 & 1 & 0 & 0 & 0 \\ 0 & 1 & 1 & 0 & 0 \\ 0 & 0 & 1 & 0 & 0 \\ 0 & 0 & 0 & -1 & 0 \\ 0 & 0 & 0 & 0 & -1\end{array}\right)$. This follows from the fact that the Jordan form of the upper left block of $\mu(\pi)$ is $\left(\begin{array}{lll}1 & 1 & 0 \\ 0 & 1 & 1 \\ 0 & 0 & 1\end{array}\right)$. Hence $G$ admits a lattice $\Gamma$.

A short calculation yields that the abelianisation of this lattice is isomorphic to $\mathbb{Z}^{2} \oplus \mathbb{Z}_{2}^{2}$, i.e. $b_{1}(G / \Gamma)=2$.

Using the assumptions of (ii), one calculates the minimal model up to generators of degree one as

$$
\begin{gathered}
\rho\left(y_{1}\right)=x_{3}, \rho\left(y_{2}\right)=x_{6}, \rho\left(y_{3}\right)=-x_{2}, \rho\left(y_{4}\right)=-x_{1} \\
d y_{1}=d y_{2}=0, d y_{3}=y_{1} y_{2}, d y_{4}=y_{2} y_{3}
\end{gathered}
$$

and $y_{1}\left(y_{3}+c\right)$ is closed but not exact for each closed $c$ of degree one.

\section{Proposition 8.4.9.}

(i) Each quotient of the Lie group $G:=G_{6.36}^{0,0}$ by a lattice is symplectic and $G$ admits a lattice $\Gamma$ with $b_{1}(G / \Gamma)=2$.

(ii) If there is a lattice in the Lie group $G$ such that the corresponding solvmanifold satisfies $b_{1}=2$ and $b_{2}=3$, then it is symplectic and not formal.

Proof. The proof of (ii) is analogous to the last one. Up to generators of degree one, the minimal model is given by

$$
\begin{gathered}
\rho\left(y_{1}\right)=x_{2}, \rho\left(y_{2}\right)=x_{6}, \rho\left(y_{3}\right)=-x_{3}, \rho\left(y_{4}\right)=x_{1}, \\
d y_{1}=d y_{2}=0, d y_{3}=y_{1} y_{2}, d y_{4}=y_{1} y_{3},
\end{gathered}
$$

and $y_{1}\left(y_{4}+c\right)$ is closed but not exact for each closed $c$ of degree one.

ad (i): Using another initial data, we argue as in the proof of Proposition 8.4.3. We now have $\mu(t)((x, y, z, v, w))=\left(x-\frac{t}{2} y^{2}, \bar{\mu}(t)((x, y, z, v, w))\right)$ with

$$
\begin{aligned}
\bar{\mu}(t)((y, z, v, w)) & =\exp ^{G L(4, \mathbb{R})}\left(t\left(\begin{array}{cccc}
0 & 0 & 0 & 0 \\
-t & 0 & 0 & 0 \\
0 & 0 & 0 & t \\
0 & 0 & -t & 0
\end{array}\right)\right)\left(\begin{array}{c}
y \\
z \\
v \\
w
\end{array}\right) \\
& =\left(\begin{array}{cccc}
1 & 0 & 0 & 0 \\
-t & 1 & 0 & 0 \\
0 & 0 & \cos (t) & \sin (t) \\
0 & 0 & -\sin (t) & \cos (t)
\end{array}\right)\left(\begin{array}{c}
y \\
z \\
v \\
w
\end{array}\right)
\end{aligned}
$$


Let $t_{1}=\pi, A:=\left(\begin{array}{cccc}1 & 1 & 0 & 0 \\ 0 & 1 & 0 & 0 \\ 0 & 0 & -1 & 0 \\ 0 & 0 & 0 & -1\end{array}\right)$ and $T:=\left(\begin{array}{cccc}0 & -\frac{1}{t_{1}} & 0 & 0 \\ 1 & 0 & 0 & 0 \\ 0 & 0 & 1 & 0 \\ 0 & 0 & 0 & 1\end{array}\right)$. Then we have $T A T^{-1}=\bar{\mu}\left(t_{1}\right)$. Denote the $i$-th column of $\mathrm{T}$ by $b_{i}$. Analogous calculations as in loc. cit. lead to a lattice generated by $\gamma_{0}:=\left(\frac{1}{t_{1}}, 0_{\mathbb{R}^{4}}\right)$ and $\gamma_{i}:=\left(b_{i 0}, b_{i}\right)$ for $i \in\{1, \ldots, 4\}$, where $b_{20} \in \mathbb{R}$ arbitrary and $b_{10}=-\frac{1}{2 t_{1}}, b_{30}=b_{40}=0$.

Obviously, this lattice is represented by

$$
\left\langle\tau, \gamma_{0}, \ldots, \gamma_{4} \mid\left[\tau, \gamma_{1}\right]=1,\left[\tau, \gamma_{2}\right]=\gamma_{1},\left[\tau, \gamma_{3}\right]=\gamma_{3}^{-2},\left[\tau, \gamma_{4}\right]=\gamma_{4}^{-2},\left[\gamma_{1}, \gamma_{2}\right]=\gamma_{0}\right\rangle
$$

and its abelianisation is $\mathbb{Z}^{2} \oplus \mathbb{Z}_{2}^{2}$, i.e. the solvmanifold's first Betti number equals two.

\section{Proposition 8.4.10.}

(i) Each quotient of the Lie group $G:=G_{6.70}^{0,0}$ by a lattice is symplectic and $G$ admits a lattice $\Gamma$ with $b_{1}(G / \Gamma)=2$.

(ii) If there is a lattice $\Gamma$ in $G$ such that $b_{1}(G / \Gamma)=2$ and $b_{2}(G / \Gamma)=3$, then $G / \Gamma$ is formal.

Proof. ad (i): By definition, we have $G=\mathbb{R} \ltimes_{\mu} N$ with $N=G_{5.1}$ and $\mu(t)=\exp ^{N} \circ \exp ^{A(\mathfrak{n})}\left(t \operatorname{ad}\left(X_{6}\right)\right) \circ \log ^{N}$, where $\left\{X_{1}, \ldots, X_{6}\right\}$ denotes a basis of $\mathfrak{g}$ as in the second row of Table 25. Note that $\left\{X_{1}, \ldots, X_{5}\right\}$ is a basis of the nilradical $\mathfrak{n}$. One computes

$$
\begin{aligned}
\mu(t)_{*} & :=d_{e}(\mu(t))=\exp ^{A(\mathfrak{n})}\left(t \operatorname{ad}\left(X_{6}\right)\right) \\
= & \left(\begin{array}{ccccc}
\cos (t) & \sin (t) & 0 & 0 & 0 \\
-\sin (t) & \cos (t) & 0 & 0 & 0 \\
0 & 0 & \cos (t) & \sin (t) & 0 \\
0 & 0 & -\sin (t) & \cos (t) & 0 \\
0 & 0 & 0 & 0 & 1
\end{array}\right) .
\end{aligned}
$$

Using $\mathfrak{n}=\left\langle X_{5}\right\rangle \ltimes_{a d}\left\langle X_{1}, \ldots, X_{4} \mid\right\rangle, \operatorname{ad}\left(X_{5}\right)\left(X_{3}\right)=-X_{1}, \operatorname{ad}\left(X_{5}\right)\left(X_{4}\right)=-X_{2}$ and $\operatorname{ad}\left(X_{5}\right)\left(X_{1}\right)=\operatorname{ad}\left(X_{5}\right)\left(X_{2}\right)=0$, we can determine the Lie group $N$.

As a smooth manifold $N$ equals $\mathbb{R}^{5}$, and the multiplication is given by

$$
(a, b, c, r, s) \cdot(x, y, z, v, w)=(a+x+c w, b+y+r w, c+z, r+v, s+w) .
$$

By Theorem 4.2, we can obtain the exponential map of $N$ as

$$
\exp ^{N}\left(x X_{1}+y X_{2}+z X_{3}+v X_{4}+w X_{5}\right)=\left(x+\frac{z w}{2}, y+\frac{v w}{2}, z, v, w\right),
$$

and obviously, this implies

$$
\log ^{N}((x, y, z, v, w))=\left(x-\frac{w z}{2}\right) X_{1}+\left(y-\frac{v w}{2}\right) X_{2}+z X_{3}+v X_{4}+w X_{5} .
$$


From $\mu(t)=\exp ^{N} \circ \mu(t)_{*} \circ \log ^{N}$ we get

$$
\begin{aligned}
\mu(t)((x, y, z, v, w))= & (\cos (t) x+\sin (t) y,-\sin (t) x+\cos (t) y, \\
& \cos (t) z+\sin (t) v,-\sin (t) z+\cos (t) v, w),
\end{aligned}
$$

and $\mu(\pi)$ preserves the lattice $\{(x, y, z, v, w) \in N \mid x, y, z, v, w \in \mathbb{Z}\}$.

The corresponding solvmanifold has $b_{1}=2$ since the abelianisation of this lattice is isomorphic to $\mathbb{Z}^{2} \oplus \mathbb{Z}_{2}{ }^{4}$.

ad (ii): Up to generators of degree two, the minimal model is given by

$$
\begin{gathered}
\rho\left(y_{1}\right)=x_{5}, \rho\left(y_{2}\right)=x_{6}, \rho\left(z_{1}\right)=x_{13}+x_{24}, \rho\left(z_{2}\right)=x_{34}, \\
d y_{1}=d y_{2}=0, d z_{1}=d z_{2}=0,
\end{gathered}
$$

hence the minimal is 2-formal. By Theorem 1.1.6, the solvmanifold is formal.

\section{Proposition 8.4.11.}

(i) $G:=G_{6.118}^{0, \pm 1,-1}$ admits a lattice such that the first Betti number of the corresponding solvmanifold equals two and the second Betti number equals five.

(ii) If there is a lattice $\Gamma$ in $G$ such that $b_{1}(G / \Gamma)=2$ and $b_{2}(G / \Gamma)=3$, then $G / \Gamma$ is symplectic and formal.

Proof. The construction of the lattices mentioned in (i) can be found in 62 . In loc. cit. $G_{6.118}^{0,1,-1}$ is denoted by $G_{3}$ and $G_{6.118}^{0,-1,-1}$ by $G_{1}$, respectively. The Betti numbers of the quotient of $G_{118}^{0,-1,-1}$ are determined explicitly. In the case of $G_{118}^{0,1,-1}$, one can make an analogous computation.

Assume there is a lattice that satisfies the condition of (ii). Up to generators of degree two, the solvmanifold's minimal model is given by

$$
\begin{gathered}
\rho\left(y_{1}\right)=x_{5}, \rho\left(y_{2}\right)=x_{6}, \rho\left(z_{1}\right)=x_{13} \pm x_{24}, \rho\left(z_{2}\right)=x_{14} \mp x_{23}, \\
d y_{1}=d y_{2}=0, d z_{1}=d z_{2}=0,
\end{gathered}
$$

hence it is 2-formal. Theorem 1.1.6 then implies formality.

Remark. $G_{6.118}^{0,-1,-1}$ is the underlying real Lie group of the unique connected and simply-connected complex three-dimensional Lie group that is solvable and not nilpotent. Its compact quotients by lattices are classified in [44, Theorem 1]. They always satisfy $b_{1}=2$ and moreover, for the Hodge number $h^{0,1}$ holds either $h^{0,1}=1$ or $h^{0,1}=3$.

Remark. Besides the groups mentioned in this section, the following solvable but not completely solvable Lie groups $G_{6 . i}$ could give rise to a symplectic solvmanifold with $b_{1}>1$. But then the cohomology class of the symplectic form cannot lie in the image of the inclusion $H^{*}\left(\bigwedge \mathfrak{g}_{6 . i}^{*}, \delta\right) \hookrightarrow H^{*}\left(G_{6 . i} / \Gamma, d\right)$.

There are the sixteen classes of groups in (13) and

$$
\begin{array}{lll}
i=9, b=0 ; & i=33, a=0 ; & i=34, a=0 ; \\
i=35, a=-b ; & i=89, s=0, \nu_{0} \neq 0 ; \quad i=92, \nu_{0} \mu_{0}=0 \\
i \in\{107,113, \ldots, 116,125,135\} . & &
\end{array}
$$




\subsection{Decomposable solvmanifolds}

The six-dimensional decomposable solvmanifolds $G / \Gamma=H_{1} / \Gamma_{1} \times H_{2} / \Gamma_{2}$ being not a nilmanifold are contained in Table 5 on page 75. Using Theorem 3.11, one can deduce the statement about the Betti numbers. The results on the existence of a symplectic form were mostly made by Campoamor-Stursberg in [9]. He examined whether the Lie algebra admits a symplectic form. Note that in [9] the symplectic forms

$$
\lambda x_{12}+\mu x_{15}+\nu x_{26}+\rho x_{34}+\sigma x_{56}, \quad \rho \neq 0, \lambda \sigma \neq \mu \nu,
$$

on $\mathfrak{g}_{5.14}^{0} \oplus \mathfrak{g}_{1}$ are absent.

Since there is a monomorphism from the Lie algebra cohomology to the solvmanifold's cohomology, the existence of a symplectic form with non-exact cubic on the Lie algebra implies the existence of such an on the solvmanifold. Recall that the Lie algebra is generated by the left-invariant one-forms on the Lie group. If the Lie algebra cohomology is isomorphic to the solvmanifold's cohomology 12 , one knows whether the solvmanifold is symplectic or not. Up to exact summands the symplectic forms are listed in Table 6 with respect to the dual of the Lie algebra's bases given in Appendix A. In the column "isom.", we mark whether there is an isomorphism of the cohomology algebras.

We do not claim that Table 5 contains all connected and simply-connected decomposable solvable and non-nilpotent Lie groups which admit a lattice - just those Lie groups admitting a lattice such that the corresponding solvmanifold is a product of lower-dimensional ones.

\section{Relations with the Lefschetz property}

We have seen in Section 1.2 that a compact Kähler manifold is formal, Hard Lefschetz and its odd-degree Betti numbers are even. Even if a manifold has a complex structure, these conditions are not sufficient as the following theorem which is mentioned in [31] shows. Recall, we have seen above that $G_{5.7}^{1,-1,-1}$ admits a lattice.

Theorem 9.1. Let $\Gamma$ be an arbitrary lattice in $G_{5.7}^{1,-1,-1}$. Then the solvmanifold $M:=G_{5.7}^{1,-1,-1} / \Gamma \times S^{1}$ is formal, Hard Lefschetz and has even odd-degree Betti numbers. Moreover, $M$ possesses a complex structure but it cannot be Kählerian.

Proof. From Proposition 7.2 .1 follows that the Lie group $G:=G_{5.7}^{1,-1,-1} \times \mathbb{R}$ possesses a lattice $\Gamma$. The Chevalley-Eilenberg complex of its Lie algebra

$$
\left\langle X_{1}, \ldots, X_{6} \mid\left[X_{1}, X_{5}\right]=X_{1},\left[X_{2}, X_{5}\right]=X_{2},\left[X_{3}, X_{5}\right]=-X_{3},\left[X_{4}, X_{5}\right]=-X_{4}\right\rangle
$$

\footnotetext{
${ }^{12}$ E.g. this happens if the Lie algebra is completely solvable or if the above monomorphism must be an isomorphism by dimension reasons.
} 
Table 5: Decomposable non-nil-solvmanifolds $G / \Gamma=H_{1} / \Gamma_{1} \times H_{2} / \Gamma_{2}$

\begin{tabular}{|c|c|c|c|c|c|}
\hline$G$ & $b_{1}(G / \Gamma)$ & $b_{2}(G / \Gamma)$ & formal & sympl. & Comment \\
\hline$G_{5.7}^{p, q, r} \times \mathbb{R}$ & 2 & 1 & yes & no & $\begin{array}{c}-1<r<q<p<1 \\
p q r \neq 0 \\
p+q+r=-1\end{array}$ \\
\hline$G_{5.7}^{p,-p,-1} \times \mathbb{R}$ & 2 & 3 & yes & yes & $p \in] 0,1[$ \\
\hline$G_{5.7}^{1,-1,-1} \times \mathbb{R}$ & 2 & 5 & yes & yes & \\
\hline$G_{5.8}^{-1} \times \mathbb{R}$ & 3 & 5 & no & yes & \\
\hline$G_{5.13}^{-1-2 q, q, r} \times \mathbb{R}$ & $\geq 2$ & $\geq 1$ & $?$ & $?$ & $\begin{array}{c}q \in[-1,0[ \\
q \neq-\frac{1}{2}, r \neq 0\end{array}$ \\
\hline$G_{5.13}^{-1,0, r} \times \mathbb{R}$ & $\geq 2$ & $\geq 3$ & $?$ & yes & $r \neq 0$ \\
\hline$G_{5.14}^{0} \times \mathbb{R}$ & $\geq 3$ & $\geq 5$ & $?$ & yes & \\
\hline$G_{5.15}^{-1} \times \mathbb{R}$ & 2 & 3 & no & yes & \\
\hline$G_{5.17}^{p,-p, r} \times \mathbb{R}$ & $\geq 2$ & $\geq 1$ & $?$ & $?$ & $p \neq 0, r \notin\{0, \pm 1\}$ \\
\hline$G_{5.17}^{p,-p, r} \times \mathbb{R}$ & $\geq 2$ & $\geq 3$ & $?$ & yes & $\begin{array}{c}(p \neq 0, r= \pm 1) \\
\text { or }(p=0, r \notin\{0, \pm 1\})\end{array}$ \\
\hline$G_{5.17}^{0,0, \pm 1} \times \mathbb{R}$ & $\geq 2$ & $\geq 5$ & $?$ & yes & \\
\hline$G_{5.18}^{0} \times \mathbb{R}$ & $\geq 2$ & $\geq 3$ & $?$ & yes & \\
\hline$G_{5.20}^{-1} \times \mathbb{R}$ & 3 & 3 & yes & no & \\
\hline$G_{5.26}^{0, \pm 1} \times \mathbb{R}$ & $\geq 3$ & $\geq 3$ & $?$ & $?$ & \\
\hline$G_{5.33}^{-1,-1} \times \mathbb{R}$ & 3 & 3 & yes & no & \\
\hline$G_{5.35}^{-2,0} \times \mathbb{R}$ & $\geq 3$ & $\geq 3$ & $?$ & $?$ & \\
\hline $\bar{c}_{4.5}^{p,-p-1} \times \mathbb{R}^{2}$ & 3 & 3 & yes & no & $p \in\left[-\frac{1}{2}, 0[\right.$ \\
\hline$G_{4.6}^{-2 p, p} \times \mathbb{R}^{2}$ & 3 & 3 & yes & no & $p>0$ \\
\hline$G_{4.8}^{-1} \times \mathbb{R}^{2}$ & 3 & 3 & yes & no & \\
\hline$G_{4.9}^{0} \times \mathbb{R}^{2}$ & 3 & 3 & yes & no & \\
\hline$\overline{G_{3.4}^{-1} \times \mathbb{R}^{3}}$ & 4 & 7 & yes & yes & \\
\hline$G_{3.5}^{0} \times \mathbb{R}^{3}$ & 4 & 7 & yes & yes & \\
\hline$G_{3.1} \times G_{3.4}^{-1}$ & 3 & 5 & no & yes & \\
\hline$G_{3.1} \times G_{3.5}^{0}$ & 3 & 5 & no & yes & \\
\hline$G_{3.4}^{-1} \times G_{3.4}^{-1}$ & 2 & 3 & yes & yes & \\
\hline$G_{3.4}^{-1} \times G_{3.5}^{0}$ & 2 & 3 & yes & yes & \\
\hline$G_{3.5}^{0} \times G_{3.5}^{0}$ & 2 & 3 & yes & yes & \\
\hline
\end{tabular}


Table 6: Symplectic forms on $G / \Gamma=H_{1} / \Gamma_{1} \times H_{2} / \Gamma_{2}$

\begin{tabular}{|c|l|c|}
\hline \hline $\mathfrak{g}$ & symplectic forms & isom. \\
\hline \hline $\mathfrak{g}_{5.7}^{p,-p,-1} \oplus \mathfrak{g}_{1}$ & $a x_{14}+b x_{23}+c x_{56}, a b c \neq 0$ & yes \\
\hline $\mathfrak{g}_{5.7}^{1,-1,-1} \oplus \mathfrak{g}_{1}$ & $a x_{13}+b x_{14}+c x_{23}+d x_{24}+e x_{56}, e(b c-a d) \neq 0$ & yes \\
\hline $\mathfrak{g}_{5.8}^{-1} \oplus \mathfrak{g}_{1}$ & $a x_{12}+b x_{15}+c x_{26}+d x_{34}+e x_{56}, d(a e-b c) \neq 0$ & yes \\
\hline $\mathfrak{g}_{5.13}^{-1,0, r} \oplus \mathfrak{g}_{1}$ & $a x_{12}+b x_{34}+c x_{56}, a b c \neq 0$ & $?$ \\
\hline $\mathfrak{g}_{5.14}^{0} \oplus \mathfrak{g}_{1}$ & $a x_{12}+b x_{15}+c x_{26}+d x_{34}+e x_{56}, d(a e-b c) \neq 0$ & $?$ \\
\hline $\mathfrak{g}_{5.15}^{-1} \oplus \mathfrak{g}_{1}$ & $a\left(x_{14}-x_{23}\right)+b x_{24}+c x_{56}, a b c \neq 0$ & yes \\
\hline $\mathfrak{g}_{5.17}^{p,-p, \pm 1} \oplus \mathfrak{g}_{1}$ & $a\left(x_{13} \pm x_{24}\right)+b\left(x_{14} \mp x_{23}\right)+c x_{56}, a b c \neq 0$ & $?$ \\
$p \neq 0$ & & \\
\hline $\mathfrak{g}_{5.17}^{0,0, r} \oplus \mathfrak{g}_{1}$ & $a x_{12}+b x_{34}+c x_{56}, a b c \neq 0$ & $?$ \\
$r \neq \pm 1$ & & $?$ \\
\hline $\mathfrak{g}_{5.17}^{0,0, \pm 1} \oplus \mathfrak{g}_{1}$ & $a x_{12}+b\left(x_{13} \pm x_{24}\right)+c\left(x_{14} \mp x_{23}\right)+d x_{34}+e x_{56}$, & $?$ \\
\hline $\mathfrak{g}_{5.18}^{0} \oplus \mathfrak{g}_{1}$ & $a\left(a d \mp\left(b^{2}+c^{2}\right)\right) \neq 0$ & $?$ \\
\hline \hline $\mathfrak{g}_{3.4}^{-1} \oplus 3 \mathfrak{g}_{1}$ & $\left.a x_{12}+b x_{24}\right)+b x_{24}+c x_{56}, a c \neq 0$ & yes \\
\hline $\mathfrak{g}_{3.5}^{0} \oplus 3 \mathfrak{g}_{1}$ & $a(d e-c f+b g) \neq 0$ & yes \\
\hline $\mathfrak{g}_{3.1} \oplus \mathfrak{g}_{3.4}^{-1}$ & $a\left(d e-c x_{36}+e x_{45}+f x_{46}+g x_{56}\right.$, & \\
\hline $\mathfrak{g}_{3.1} \oplus \mathfrak{g}_{3.5}^{0}$ & $a x_{12}+b x_{13}+c x_{35}+d x_{36}+e x_{45}+f x_{46}+g x_{56}$, & yes \\
\hline $\mathfrak{g}_{3.4}^{-1} \oplus \mathfrak{g}_{3.4}^{-1}$ & $a x_{12}+b x_{36}+c x_{45}, a b c \neq 0$ & \\
\hline $\mathfrak{g}_{3.4}^{-1} \oplus \mathfrak{g}_{3.5}^{0}$ & $a x_{12}+b x_{36}+c x_{45}, a b c \neq 0$ & yes \\
\hline $\mathfrak{g}_{3.5}^{0} \oplus \mathfrak{g}_{3.5}^{0}$ & $a x_{12}+b x_{36}+c x_{45}, a b c \neq 0$ & yes \\
\hline
\end{tabular}


is given by

$$
\delta x_{1}=-x_{15}, \delta x_{2}=-x_{25}, \delta x_{3}=x_{35}, \delta x_{4}=x_{45}, \delta x_{5}=\delta x_{6}=0
$$

where $\left\{x_{1}, \ldots, x_{6}\right\}$ is a basis of the left-invariant one-forms on $G$. Since $G$ is completely solvable, Theorem 3.11 (ii) enables us to compute the cohomology of $M$ as

$$
\begin{aligned}
H^{1}(M, \mathbb{R}) & =\left\langle\left[x_{5}\right],\left[x_{6}\right]\right\rangle \\
H^{2}(M, \mathbb{R}) & =\left\langle\left[x_{13}\right],\left[x_{14}\right],\left[x_{23}\right],\left[x_{24}\right],\left[x_{56}\right]\right\rangle \\
H^{3}(M, \mathbb{R}) & =\left\langle\left[x_{135}\right],\left[x_{136}\right],\left[x_{145}\right],\left[x_{146}\right],\left[x_{235}\right],\left[x_{236}\right],\left[x_{245}\right],\left[x_{246}\right]\right\rangle \\
H^{4}(M, \mathbb{R}) & =\left\langle\left[x_{1234}\right],\left[x_{1356}\right],\left[x_{1456}\right],\left[x_{2356}\right],\left[x_{2456}\right]\right\rangle \\
H^{5}(M, \mathbb{R}) & =\left\langle\left[x_{12345}\right],\left[x_{12346}\right]\right\rangle .
\end{aligned}
$$

Let $[\omega] \in H^{2}(M, \mathbb{R})$ represent a symplectic form on $M$. A short calculation shows that there are $a, b, c, d, e \in \mathbb{R}$ with $e(b c-a d) \neq 0$ and

$$
[\omega]=a\left[x_{13}\right]+b\left[x_{14}\right]+c\left[x_{23}\right]+d\left[x_{24}\right]+e\left[x_{56}\right] .
$$

Since $\left[x_{5}\right] \cup[\omega]^{2}=2(b c-d e)\left[x_{12345}\right] \neq 0$ and $\left[x_{6}\right] \cup[\omega]^{2}=2(b c-d e)\left[x_{12346}\right] \neq 0$, the homomorphism $L^{2}: H^{1}(M, \mathbb{R}) \rightarrow H^{5}(M, \mathbb{R})$ is an isomorphism.

In the basis (15), the homomorphism $L^{1}: H^{2}(M, \mathbb{R}) \rightarrow H^{4}(M, \mathbb{R})$ is represented by the matrix $\left(\begin{array}{ccccc}-d & c & -b & -a & 0 \\ e & 0 & 0 & 0 & a \\ 0 & e & 0 & 0 & b \\ 0 & 0 & e & 0 & c \\ 0 & 0 & 0 & e & d\end{array}\right)$ which has $2 e^{3}(a d-b c) \neq 0$ as determinant, hence $M$ is Hard Lefschetz.

We define an almost complex structure $J$ on $G$ by

$$
J X_{1}=X_{2}, J X_{2}=-X_{1}, J X_{3}=X_{4}, J X_{4}=-X_{3}, J X_{5}=X_{6}, J X_{6}=-X_{5},
$$

which induces an almost complex structure on $M$. It is easy to see that the Nijenhuis tensor vanishes, hence $M$ is a complex manifold.

$M$ is a non-toral solvmanifold which is a quotient of a completely solvable Lie group. Therefore, $M$ cannot be Kählerian by Theorem 3.13 ,

The authors of [34] considered the relations between the above three properties for closed symplectic manifolds. We want to try to complete [34, Theorem 3.1 Table 1] in the case of symplectic solvmanifolds. Actually, the mentioned table deals with symplectically aspherical closed manifolds, but note that symplectic solvmanifolds are symplectically aspherical.

We start our investigations by the examination of the Lefschetz property in dimension four. 
Theorem 9.2. A four-dimensional symplectic solvmanifold is not (Hard) Lefschetz if and only if it is a non-toral nilmanifold. Especially, the (Hard) Lefschetz property is independent of the choice of the symplectic form.

Proof. By Theorem 6.2, there are five classes of four-dimensional symplectic solvmanifolds. Three of them are nilmanifolds and satisfy the claim by Corollary 2.10 .

There remain two non-nilmanifolds to consider. We start with a quotient of the Lie group which has $\mathfrak{g}_{3.1}^{-1} \oplus \mathfrak{g}_{1}$ as Lie algebra, see Table 8. The Lie group is completely solvable, hence the Lie algebra cohomology is isomorphic to the solvmanifold's cohomology. If $x_{1}, \ldots, x_{4}$ denote the left-invariant one-forms which are dual to the basis given in Table 8, one computes

$$
H^{1}=\left\langle\left[x_{3}\right],\left[x_{4}\right]\right\rangle, \quad H^{2}=\left\langle\left[x_{12}\right],\left[x_{34}\right]\right\rangle, \quad H^{3}=\left\langle\left[x_{123}\right],\left[x_{124}\right]\right\rangle .
$$

The class representing a symplectic form must be of the form $\left[a x_{12}+b x_{34}\right]$ with $a, b \neq 0$ and obviously, the Lefschetz map with respect to this class is an isomorphism.

Now, consider a solvmanifold $G / \Gamma$ such that the Lie algebra of $G$ is $\mathfrak{g}_{3.5}^{0} \oplus \mathfrak{g}_{1}$ and $b_{1}(G / \Gamma)=2$. A short computation yields that the Lie algebra cohomology of $\mathfrak{g}_{3.5} \oplus \mathfrak{g}_{1}$ is the same as in (16). Since $G / \Gamma$ is compact and parallelisable, we see further $b_{i}(G / \Gamma)=2$ for $i \in\{1,2,3\}$, and Theorem 3.11 (i) implies that (16) also gives the cohomology of $G / \Gamma$. We have yet seen that a symplectic four-manifold with this cohomology is Hard Lefschetz.

Denote $K T$ "the" four-dimensional symplectic nilmanifold with $b_{1}(K T)=3$. We have seen that $K T$ is not formal and not Lefschetz. Its square has the following properties:

Theorem 9.3 ([34]). KT $\times K T$ is not formal, not Lefschetz and has even odddegree Betti numbers.

Next, we are looking for an example of a formal manifold that is not Lefschetz and has even odd degree Betti numbers resp. an odd odd degree Betti number.

Theorem 9.4. The Lie group $G_{6.78}$ admits a lattice $\Gamma$, see above. $M:=G_{6.78} / \Gamma$ is a formal solvmanifold with $b_{1}(M)=1$ that admits a symplectic form $\omega$ such that $(M, \omega)$ is not Hard Lefschetz. Moreover, $(M \times M, \omega \times \omega)$ is a formal symplectic manifold with even odd degree Betti numbers that is not Hard Lefschetz.

Proof. By Theorem 8.3.4, $M$ is a formal symplectic manifold with Betti numbers $b_{1}(M)=b_{2}(M)=1$. Note that this implies that $M \times M$ is symplectic and formal (the latter property by Proposition 1.1.7).

Corollary 1.2.4 forces $M$ to be not Lefschetz and since [21, Proposition 4.2] says that a product is Lefschetz if and only if both factors are Lefschetz, $M \times M$ is not Lefschetz. 
$M$ is a six-dimensional solvmanifold and so it is parallelisable. Hence the fact $b_{0}(M)=b_{1}(M)=b_{2}(M)=1$ implies $b_{3}(M)=2$. This and Poincaré Duality imply $b_{1}(M \times M)=b_{11}(M \times M)=2, b_{3}(M \times M)=b_{9}(M \times M)=6$ and $b_{5}(M \times M)=b_{7}(M \times M)=4$.

In 1990, Benson and Gordon [4, Example 3] constructed an eight-dimensional non-exact symplectic and completely solvable Lie algebra that does not satisfy the Hard Lefschetz property, but they did not know whether the corresponding connected and simply-connected Lie group $G^{B G}$ admits a lattice.

Fernández, de León and Saralegui computed in [20, Proposition 3.2] the minimal model of the complex of the left-invariant differential forms on $G^{B G}$. It is formal and its cohomology of odd degree is even-dimensional. If $G^{B G}$ admits a lattice, by completely solvability, the corresponding solvmanifold would be a symplectic and formal manifold with even odd degree Betti numbers that violates the Hard Lefschetz property.

In 2000, Tralle [57] claimed that a lattice does not exist but Sawai and Yamada noted 2005 Tralle's proof would contain calculatory errors and constructed a lattice [53, Theorem 1]. This proves the next theorem.

Theorem 9.5. There exists an eight-dimensional symplectic and formal solvmanifold $M^{B G}$ with even odd degree Betti numbers that is not Hard Lefschetz.

We sum up the above results in Table 7 on page 79, It is an enlargement of [34, Theorem 3.1 Table 1].

Table 7: Relations of the Kähler properties

\begin{tabular}{|c|c|c|c|}
\hline \hline Formality & Hard Lefschetz & $b_{2 i+1} \equiv 0(2)$ & Example \\
\hline \hline yes & yes & yes & Kähler \\
\hline yes & yes & no & impossible \\
\hline yes & no & yes & $M^{B G}, G_{6.78} / \Gamma \times G_{6.78} / \Gamma$ \\
\hline yes & no & no & $G_{6.78} / \Gamma$ \\
\hline no & yes & yes & $?$ \\
\hline no & yes & no & impossible \\
\hline no & no & yes & $K T \times K T$ \\
\hline no & no & no & $K T$ \\
\hline
\end{tabular}

Unfortunately, the missing example does not arise among the six-dimensional solvmanifolds. In order to see this, one has to examine which of them satisfy the (Hard) Lefschetz property. We briefly mention the results.

By Corollary 1.2.4, a manifold with odd first Betti number cannot be Lefschetz. We now examine such indecomposable solvmanifolds whose first Betti number is even; in the completely solvable case, these are quotients of $G_{6.3}^{-1}, G_{6.21}^{0}$ and $G_{6.54}^{-1}$. 
The proof of the next two propositions is done analogous as that of Theorem 9.1. By complete solvability, we know the solvmanifolds' cohomology and all possible symplectic forms were determined in the proof of Proposition 8.4.1, Therefore, one can compute the image of the Lefschetz maps.

Proposition 9.6. Let a lattice in $G_{6.3}^{-1}$ or $G_{6.21}^{0}$ be given. (Wee have seen above that such exists.) Then the corresponding (non-formal) symplectic solvmanifold (with $b_{1}=2, b_{2}=3$ ) is not Lefschetz, independent of the choice of the symplectic form.

Proposition 9.7. Let a lattice in $G_{6.54}^{-1}$ be given. (Such exists by Proposition 8.4.6.) The corresponding (non-formal) symplectic solvmanifold (with $b_{1}=2$, $\left.b_{2}=5\right)$ is Lefschetz but not Hard Lefschetz, independent of the choice of the symplectic form.

Remark. The existence of a lattice in $G_{6.54}^{-1}$ was proven by Fernández, de Léon and Saralegui in [20]. They also computed the Betti numbers of the corresponding solvmanifold, showed that it is not formal and does not satisfy the Hard Lefschetz property with respect to a certain symplectic form. Moreover, Fernández and Muñoz proved in [21, Example 3] that the manifold is Lefschetz. (Analogous calculations work for other symplectic forms.)

In the non-completely solvable case, the situation becomes a little more complicated. If we are willing to make a statement about the Lefschetz property, we have to know the cohomology and need therefore assumptions on the Betti numbers.

Proposition 9.8. If there is a lattice in one of the non-completely solvable groups $G_{6 . i}^{0,0}, i \in\{10,36,70\}$ resp. $G_{6.118}^{0, \pm 1,-1}$ such that the cohomology of the corresponding solvmanifold $M_{i}$ is isomorphic to the Lie algebra cohomology of $\mathfrak{g}_{6 . i}$ (i.e. the cohomology is as small as possible), then one computes that the following hold, independent of the choice of the symplectic forms provided by Proposition 8.4.1:

- $M_{10}$ and $M_{36}$ are not formal and not Lefschetz.

- $M_{70}$ is formal and Lefschetz but not Hard Lefschetz.

- $M_{118}$ is formal and Hard Lefschetz.

(The statements on formality follow from the propositions at the end of Section 8.4.)

Finally, we consider the decomposable symplectic solvmanifolds listed in Table 5 
Proposition 9.9. Let $G / \Gamma=H_{1} / \Gamma_{1} \times H_{2} / \Gamma_{2}$ be one of the symplectic solvmanifolds listed in Table 5 such that in the corresponding row of the table arises no $\geq$-sign.

Then $G / \Gamma$ is formal if and only if it is Hard Lefschetz (independent of the special choice of the symplectic form).

Sketch of the proof. One has an isomorphism from the Lie algebra cohomology to the solvmanifold's cohomology for each manifold as in the theorem. Then an explicit calculation as in the proof of Theorem 9.1 yields that the Hard Lefschetz manifolds among the considered are exactly the formal ones.

Note, if $b_{1}$ is not even, the claim follows directly from Theorem 1.2.3.

Remark. $G_{5.15}^{-1} / \Gamma_{1} \times S^{1}$ is Lefschetz. The other manifolds in the last proposition are even not Lefschetz if they are not Hard Lefschetz.

$G_{5.15}^{-1} / \Gamma_{1} \times S^{1}$ is a Lefschetz manifold that is not formal and has even odd degree Betti numbers.

A similar result as the last proposition holds for the manifolds in Table 5 such that in the corresponding row of the table arises a $\geq$-sign. But we again must make an assumption that enables us to compute the whole cohomology.

\section{Proposition 9.10.}

(i) Let $M=G_{5 . i} / \Gamma \times \mathbb{R} / \mathbb{Z}$ be a symplectic manifold such that one of the following conditions holds:

a) $i=13$ with $q=0$ and $b_{1}(M)=2$ as well as $b_{2}(M)=3$,

b) $i=17$ with $p \neq 0, r= \pm 1$ and $b_{1}(M)=2$ as well as $b_{2}(M)=3$,

c) $i=17$ with $p=0, r \notin\{0, \pm 1\}$ and $b_{1}(M)=2$ as well as $b_{2}(M)=3$,

d) $i=17$ with $p=0, r= \pm 1$ and $b_{1}(M)=2$ as well as $b_{2}(M)=5$.

Then $M$ is formal and Hard Lefschetz (independent of the special choice of the symplectic form).

(ii) Let $\Gamma$ be a lattice in $G_{5.14}^{0}$ such that $M=G_{5.14}^{0} / \Gamma \times \mathbb{R} / \mathbb{Z}$ satisfies $b_{1}(M)=3$ and $b_{2}(M)=5$.

Then $M$ is not formal and not Lefschetz (independent of the special choice of the symplectic form).

(iii) Let $\Gamma$ be a lattice in $G_{5.18}^{0}$ such that $M=G_{5.18}^{0} / \Gamma \times \mathbb{R} / \mathbb{Z}$ satisfies $b_{1}(M)=2$ and $b_{2}(M)=3$.

Then $M$ is not formal and Lefschetz but not Hard Lefschetz (independent of the special choice of the symplectic form). 


\section{A Lists of Lie Algebras}

In Table 8, we give the isomorphism classes of Lie algebras of the simplyconnected solvable Lie groups up to dimension four that possesses lattices. The designation $\mathfrak{g}_{i, j}$ means the $j$-th indecomposable solvable Lie algebra of dimension $i$. The choice of the integer $j$ bases on the notation of [41]. The superscripts, if any, give the values of the continuous parameters on which the algebra depends.

Table 8: Solvmanifolds up to dimension four

\begin{tabular}{|c|c|c|}
\hline \hline & {$\left[X_{i}, X_{j}\right]$} & cpl. solv. \\
\hline \hline $\mathfrak{g}_{1}$ & {$\left[X_{2}, X_{3}\right]=X_{1}$} & abelian \\
\hline \hline $2 \mathfrak{g}_{1}$ & {$\left[X_{1}, X_{3}\right]=X_{1},\left[X_{2}, X_{3}\right]=-X_{2}$} & abelian \\
\hline \hline $3 \mathfrak{g}_{1}$ & {$\left[X_{1}, X_{3}\right]=-X_{2},\left[X_{2}, X_{3}\right]=X_{1}$} & abelian \\
\hline $\mathfrak{g}_{3.1}$ & {$\left[X_{2}, X_{3}\right]=X_{1}$} & nilpotent \\
\hline $\mathfrak{g}_{3.4}^{-1}$ & {$\left[X_{1}, X_{3}\right]=X_{1},\left[X_{2}, X_{3}\right]=-X_{2}$} & yes \\
\hline $\mathfrak{g}_{3.5}^{0}$ & {$\left[X_{1}, X_{3}\right]=-X_{2},\left[X_{2}, X_{3}\right]=X_{1}$} & abelian \\
\hline \hline $4 \mathfrak{g}_{1}$ & {$\left[X_{2}, X_{4}\right]=X_{1},\left[X_{3}, X_{4}\right]=X_{2}$} & nilpotent \\
\hline $\mathfrak{g}_{3.1} \oplus \mathfrak{g}_{1}$ & {$\left[X_{1}, X_{4}\right]=X_{1},\left[X_{2}, X_{4}\right]=p X_{2}$,} & nes \\
\hline $\mathfrak{g}_{3.4}^{-1} \oplus \mathfrak{g}_{1}$ & {$\left[X_{3}, X_{4}\right]=(-p-1) X_{3},-\frac{1}{2} \leq p<0$} & nilpotent \\
\hline $\mathfrak{g}_{3.5}^{0} \oplus \mathfrak{g}_{1}$ & {$\left[X_{1}, X_{4}\right]=-2 p X_{1},\left[X_{2}, X_{4}\right]=p X_{2}-X_{3}$,} & yes \\
\hline $\mathfrak{g}_{4.1}$ & {$\left[X_{3}, X_{4}\right]=X_{2}+p X_{3}, p>0$} & no \\
\hline $\mathfrak{g}_{4.5}^{p,-p-1}$ & {$\left[X_{2}, X_{3}\right]=X_{1},\left[X_{2}, X_{4}\right]=X_{2},\left[X_{3}, X_{4}\right]=-X_{3}$} & yes \\
\hline $\mathfrak{g}_{4.6}^{-2 p, p}$ & {$\left[X_{2}, X_{3}\right]=X_{1},\left[X_{2}, X_{4}\right]=-X_{3},\left[X_{3}, X_{4}\right]=X_{2}$} & no \\
\hline $\mathfrak{g}_{4.8}^{-1}$ & & \\
\hline $\mathfrak{g}_{4.9}^{0}$ & & \\
\hline
\end{tabular}

We do not claim that the corresponding Lie groups admit a lattice for all parameters. We just know that there exist such for certain parameters!

The set of non-isomorphic five dimensional nilpotent Lie algebras is exhausted by three types of decomposable algebras and six indecomposables which are listed in the next table. The designation is taken from [42]. 
Table 9: 5-dimensional nilpotent algebras

\begin{tabular}{|c|c|}
\hline \hline & {$\left[X_{i}, X_{j}\right]$} \\
\hline \hline $5 \mathfrak{g}_{1}$ & abelian \\
\hline $\mathfrak{g}_{3.1} \oplus 2 \mathfrak{g}_{1}$ & {$\left[X_{2}, X_{3}\right]=X_{1}$} \\
\hline $\mathfrak{g}_{4.1} \oplus \mathfrak{g}_{1}$ & {$\left[X_{2}, X_{4}\right]=X_{1},\left[X_{3}, X_{4}\right]=X_{2}$} \\
\hline $\mathfrak{g}_{5.1}$ & {$\left[X_{3}, X_{5}\right]=X_{1},\left[X_{4}, X_{5}\right]=X_{2}$} \\
\hline $\mathfrak{g}_{5.2}$ & {$\left[X_{2}, X_{5}\right]=X_{1},\left[X_{3}, X_{5}\right]=X_{2},\left[X_{4}, X_{5}\right]=X_{3}$} \\
\hline $\mathfrak{g}_{5.3}$ & {$\left[X_{2}, X_{4}\right]=X_{3},\left[X_{2}, X_{5}\right]=X_{1},\left[X_{4}, X_{5}\right]=X_{2}$} \\
\hline $\mathfrak{g}_{5.4}$ & {$\left[X_{2}, X_{4}\right]=X_{1},\left[X_{3}, X_{5}\right]=X_{1}$} \\
\hline $\mathfrak{g}_{5.5}$ & {$\left[X_{3}, X_{4}\right]=X_{1},\left[X_{2}, X_{5}\right]=X_{1},\left[X_{3}, X_{5}\right]=X_{2}$} \\
\hline $\mathfrak{g}_{5.6}$ & {$\left[X_{3}, X_{4}\right]=X_{1},\left[X_{2}, X_{5}\right]=X_{1},\left[X_{3}, X_{5}\right]=X_{2},\left[X_{4}, X_{5}\right]=X_{3}$} \\
\hline
\end{tabular}

There are 24 classes of solvable and non-nilpotent decomposable Lie algebras in dimension five. The unimodular among them are the ones in Table 10.

Table 10: 5-dimensional decomposable unimodular non-nilpotent algebras

\begin{tabular}{|c|c|c|}
\hline \hline & {$\left[X_{i}, X_{j}\right]$} & cpl. solv. \\
\hline \hline $\mathfrak{g}_{3.4}^{-1} \oplus 2 \mathfrak{g}_{1}$ & {$\left[X_{1}, X_{3}\right]=X_{1},\left[X_{2}, X_{3}\right]=-X_{2}$} & yes \\
\hline $\mathfrak{g}_{3.5}^{0} \oplus 2 \mathfrak{g}_{1}$ & {$\left[X_{1}, X_{3}\right]=-X_{2},\left[X_{2}, X_{3}\right]=X_{1}$} & no \\
\hline $\mathfrak{g}_{4.2}^{-2} \oplus \mathfrak{g}_{1}$ & {$\left[X_{1}, X_{4}\right]=-2 X_{1},\left[X_{2}, X_{4}\right]=X_{2}$,} & yes \\
& {$\left[X_{3}, X_{4}\right]=X_{2}+X_{3}$} & \\
\hline $\mathfrak{g}_{4.5}^{p,-p-1} \oplus \mathfrak{g}_{1}$ & {$\left[X_{1}, X_{4}\right]=X_{1},\left[X_{2}, X_{4}\right]=p X_{2}$,} & yes \\
& {$\left[X_{3}, X_{4}\right]=(-p-1) X_{3},-\frac{1}{2} \leq p<0$} & \\
\hline $\mathfrak{g}_{4.6}^{-2 p, p} \oplus \mathfrak{g}_{1}$ & {$\left[X_{1}, X_{4}\right]=-2 p X_{1},\left[X_{2}, X_{4}\right]=p X_{2}-X_{3}$,} & no \\
& {$\left[X_{3}, X_{4}\right]=X_{2}+p X_{3}, p>0$} & \\
\hline $\mathfrak{g}_{4.8}^{-1} \oplus \mathfrak{g}_{1}$ & {$\left[X_{2}, X_{3}\right]=X_{1},\left[X_{2}, X_{4}\right]=X_{2},\left[X_{3}, X_{4}\right]=-X_{3}$} & yes \\
\hline $\mathfrak{g}_{4.9}^{0} \oplus \mathfrak{g}_{1}$ & {$\left[X_{2}, X_{3}\right]=X_{1},\left[X_{2}, X_{4}\right]=-X_{3},\left[X_{3}, X_{4}\right]=X_{2}$} & no \\
\hline
\end{tabular}

Except for $\mathfrak{g}_{4.2} \oplus \mathfrak{g}_{1}$, to each class of algebras there is a connected and simplyconnected solvable Lie group admitting a lattice and has a Lie algebra belonging to the class.

Mubarakzjanov's list in [42] contains 33 classes of five-dimensional indecomposable non-nilpotent solvable Lie algebras, namely $\mathfrak{g}_{5.7}, \ldots, \mathfrak{g}_{5.39}$. We list the unimodular among them in Tables 11 to 14.

Note that there is a minor misprint in [42] which has been corrected in the list below. 
Table 11: 5-dimensional indecomposable unimodular almost abelian algebras

\begin{tabular}{|c|c|c|}
\hline & {$\left[X_{i}, X_{j}\right]$} & cpl. solv. \\
\hline $\mathfrak{g}_{5.7}^{p, q, r}$ & $\begin{array}{c}\left.X_{1}, X_{5}\right]=X_{1},\left[X_{2}, X_{5}\right]=p X_{2}, \\
{\left[X_{3}, X_{5}\right]=q X_{3},\left[X_{4}, X_{5}\right]=r X_{4}} \\
-1 \leq r \leq q \leq p \leq 1, p q r \neq 0, p+q+r=-1\end{array}$ & yes \\
\hline $\mathfrak{g}_{5.8}^{-1}$ & {$\left[X_{2}, X_{5}\right]=X_{1},\left[X_{3}, X_{5}\right]=X_{3},\left[X_{4}, X_{5}\right]=-X_{4}$} & yes \\
\hline $\mathfrak{g}_{5.9}^{p,-2-p}$ & $\begin{array}{c}{\left[X_{1}, X_{5}\right]=X_{1},\left[X_{2}, X_{5}\right]=X_{1}+X_{2},\left[X_{3}, X_{5}\right]=p X_{3}} \\
{\left[X_{4}, X_{5}\right]=(-2-p) X_{4}, p \geq-1}\end{array}$ & yes \\
\hline $\mathfrak{g}_{5.11}^{-3}$ & $\begin{array}{c}{\left[X_{1}, X_{5}\right]=X_{1},\left[X_{2}, X_{5}\right]=X_{1}+X_{2}} \\
{\left[X_{3}, X_{5}\right]=X_{2}+X_{3},\left[X_{4}, X_{5}\right]=-3 X_{4}}\end{array}$ & yes \\
\hline $\mathfrak{g}_{5.13}^{-1-2 q, q, r}$ & $\begin{array}{c}{\left[X_{1}, X_{5}\right]=X_{1},\left[X_{2}, X_{5}\right]=(-1-2 q) X_{2}} \\
{\left[X_{3}, X_{5}\right]=q X_{3}-r X_{4},\left[X_{4}, X_{5}\right]=r X_{3}+q X_{4}} \\
-1 \leq q \leq 0, q \neq-\frac{1}{2}, \quad r \neq 0\end{array}$ & no \\
\hline $\mathfrak{g}_{5.14}^{0}$ & $\left.X_{2}, X_{5}\right]=X_{1},\left[X_{3}, X_{5}\right]=-X_{4},\left[X_{4}, X_{5}\right]=X_{3}$ & no \\
\hline $\mathfrak{g}_{5.15}^{-1}$ & $\begin{array}{c}{\left[X_{1}, X_{5}\right]=X_{1},\left[X_{2}, X_{5}\right]=X_{1}+X_{2}} \\
{\left[X_{3}, X_{5}\right]=-X_{3},\left[X_{4}, X_{5}\right]=X_{3}-X_{4}}\end{array}$ & yes \\
\hline $\mathfrak{g}_{5.16}^{-1, q}$ & $\begin{array}{c}{\left[X_{1}, X_{5}\right]=X_{1},\left[X_{2}, X_{5}\right]=X_{1}+X_{2}} \\
{\left[X_{3}, X_{5}\right]=-X_{3}-q X_{4},\left[X_{4}, X_{5}\right]=q X_{3}-X_{4}} \\
q \neq 0\end{array}$ & no \\
\hline $\mathfrak{g}_{5.17}^{p,-p, r}$ & $\begin{array}{c}{\left[X_{1}, X_{5}\right]=p X_{1}-X_{2},\left[X_{2}, X_{5}\right]=X_{1}+p X_{2}} \\
{\left[X_{3}, X_{5}\right]=-p X_{3}-r X_{4},\left[X_{4}, X_{5}\right]=r X_{3}-p X_{4}} \\
r \neq 0\end{array}$ & no \\
\hline $\mathfrak{g}_{5.18}^{0}$ & $\begin{array}{c}{\left[X_{1}, X_{5}\right]=-X_{2},\left[X_{2}, X_{5}\right]=X_{1}} \\
{\left[X_{3}, X_{5}\right]=X_{1}-X_{4},\left[X_{4}, X_{5}\right]=X_{2}+X_{3}}\end{array}$ & no \\
\hline
\end{tabular}

Table 12: 5-dimensional indecomposable unimodular algebras with nilradical $\mathfrak{g}_{3.1} \oplus \mathfrak{g}_{1}$

\begin{tabular}{|c|c|c|}
\hline \hline & {$\left[X_{i}, X_{j}\right]$} & cpl. solv. \\
\hline \hline $\mathfrak{g}_{5.19}^{p,-2 p-2}$ & {$\left[X_{2}, X_{3}\right]=X_{1},\left[X_{1}, X_{5}\right]=(1+p) X_{1},\left[X_{2}, X_{5}\right]=X_{2}$,} & yes \\
& {$\left[X_{3}, X_{5}\right]=p X_{3},\left[X_{4}, X_{5}\right]=(-2 p-2) X_{4}, p \neq-1$} & \\
\hline $\mathfrak{g}_{5.20}^{-1}$ & {$\left[X_{2}, X_{3}\right]=X_{1},\left[X_{2}, X_{5}\right]=X_{2},\left[X_{3}, X_{5}\right]=-X_{3}$,} & yes \\
{$\left[X_{4}, X_{5}\right]=X_{1}$} & \\
\hline $\mathfrak{g}_{5.23}^{-4}$ & {$\left[X_{2}, X_{3}\right]=X_{1},\left[X_{1}, X_{5}\right]=2 X_{1},\left[X_{2}, X_{5}\right]=X_{2}+X_{3}$,} & yes \\
& {$\left[X_{3}, X_{5}\right]=X_{3},\left[X_{4}, X_{5}\right]=-4 X_{4}$} & no \\
\hline $\mathfrak{g}_{5.25}^{p, 4 p}$ & $\begin{array}{c}{\left[X_{2}, X_{3}\right]=X_{1},\left[X_{1}, X_{5}\right]=2 p X_{1},\left[X_{2}, X_{5}\right]=p X_{2}+X_{3},} \\
{\left[X_{3}, X_{5}\right]=-X_{2}+p X_{3},\left[X_{4}, X_{5}\right]=-4 p X_{4}, p \neq 0}\end{array}$ \\
\hline $\mathfrak{g}_{5.26}^{0, \varepsilon}$ & {$\left[X_{2}, X_{3}\right]=X_{1},\left[X_{2}, X_{5}\right]=X_{3},\left[X_{3}, X_{5}\right]=-X_{2}$,} & no \\
\hline $\mathfrak{g}_{5.28}^{-\frac{3}{2}}$ & {$\left[X_{4}, X_{5}\right]=\varepsilon X_{1}, \varepsilon= \pm 1$} & \\
& {$\left[X_{2}, X_{3}\right]=X_{1},\left[X_{1}, X_{5}\right]=-\frac{1}{2} X_{1},\left[X_{2}, X_{5}\right]=-\frac{3}{2} X_{2}$,} & yes \\
{$\left[X_{3}, X_{5}\right]=X_{3}+X_{4},\left[X_{4}, X_{5}\right]=X_{4}$} & \\
\hline
\end{tabular}


Table 13: 5-dimensional indecomposable unimodular algebras with nilradical $\mathfrak{g}_{4.1}$

\begin{tabular}{|c|c|c|}
\hline \hline & {$\left[X_{i}, X_{j}\right]$} & cpl. solv. \\
\hline \hline $\mathfrak{g}_{5.30}^{-\frac{4}{3}}$ & {$\left[X_{2}, X_{4}\right]=X_{1},\left[X_{3}, X_{4}\right]=X_{2},\left[X_{1}, X_{5}\right]=\frac{2}{3} X_{1}$,} & \multirow{2}{*}{ yes } \\
& {$\left[X_{2}, X_{5}\right]=-\frac{1}{3} X_{2},\left[X_{3}, X_{5}\right]=-\frac{4}{3} X_{3},\left[X_{4}, X_{5}\right]=X_{4}$,} & \\
\hline
\end{tabular}

Table 14: 5-dimensional indecomposable unimodular algebras with nilradical $3 \mathfrak{g}_{1}$

\begin{tabular}{|c|c|c|}
\hline \hline & {$\left[X_{i}, X_{j}\right]$} & cpl. solv. \\
\hline \hline $\mathfrak{g}_{5.33}^{-1,-1}$ & {$\left[X_{1}, X_{4}\right]=X_{1},\left[X_{3}, X_{4}\right]=-X_{3}$,} & yes \\
& {$\left[X_{2}, X_{5}\right]=X_{2},\left[X_{3}, X_{5}\right]=-X_{3}$} & \\
\hline $\mathfrak{g}_{5.35}^{-2,0}$ & {$\left[X_{1}, X_{4}\right]=-2 X_{1},\left[X_{2}, X_{4}\right]=X_{2},\left[X_{3}, X_{4}\right]=X_{3}$} & no \\
& {$\left[X_{2}, X_{5}\right]=-X_{3},\left[X_{3}, X_{5}\right]=X_{2}$} & \\
\hline
\end{tabular}

There are ten classes of decomposable nilpotent Lie algebras in dimension six: $6 \mathfrak{g}_{1}, \mathfrak{g}_{3.1} \oplus 3 \mathfrak{g}_{1}, 2 \mathfrak{g}_{3.1}, \mathfrak{g}_{4.1} \oplus 2 \mathfrak{g}_{1}$ and $\mathfrak{g}_{5 . i} \oplus \mathfrak{g}_{1}$ for $i \in\{1, \ldots 6\}$.

Tables 15 and [16] contain the six-dimensional indecomposable nilpotent real Lie algebras. They base on Morozov's classification in [39], where nilpotent algebras over a field of characteristic zero are determined. Note that over $\mathbb{R}$, there is only one isomorphism class of Morozov's indecomposable type 5 resp. type 10 and type 14 resp. 18 splits into two non-isomorphic ones.

Table 15: 6-dimensional indecomposable nilpotent algebras

\begin{tabular}{|c|c|}
\hline \hline & {$\left[X_{i}, X_{j}\right]$} \\
\hline \hline $\mathfrak{g}_{6 . N 1}$ & {$\left[X_{1}, X_{2}\right]=X_{3},\left[X_{1}, X_{3}\right]=X_{4},\left[X_{1}, X_{5}\right]=X_{6}$} \\
\hline $\mathfrak{g}_{6 . N 2}$ & {$\left[X_{1}, X_{2}\right]=X_{3},\left[X_{1}, X_{3}\right]=X_{4},\left[X_{1}, X_{4}\right]=X_{5},\left[X_{1}, X_{5}\right]=X_{6}$} \\
\hline $\mathfrak{g}_{6 . N 3}$ & {$\left[X_{1}, X_{2}\right]=X_{6},\left[X_{1}, X_{3}\right]=X_{4},\left[X_{2}, X_{3}\right]=X_{5}$} \\
\hline $\mathfrak{g}_{6 . N 4}$ & {$\left[X_{1}, X_{2}\right]=X_{5},\left[X_{1}, X_{3}\right]=X_{6},\left[X_{2}, X_{4}\right]=X_{6}$} \\
\hline $\mathfrak{g}_{6 . N 5}$ & {$\left[X_{1}, X_{3}\right]=X_{5},\left[X_{1}, X_{4}\right]=X_{6},\left[X_{2}, X_{3}\right]=-X_{6},\left[X_{2}, X_{4}\right]=X_{5}$} \\
\hline $\mathfrak{g}_{6 . N 6}$ & {$\left[X_{1}, X_{2}\right]=X_{6},\left[X_{1}, X_{3}\right]=X_{4},\left[X_{1}, X_{4}\right]=X_{5},\left[X_{2}, X_{3}\right]=X_{5}$} \\
\hline $\mathfrak{g}_{6 . N 7}$ & {$\left[X_{1}, X_{3}\right]=X_{4},\left[X_{1}, X_{4}\right]=X_{5},\left[X_{2}, X_{3}\right]=X_{6}$} \\
\hline $\mathfrak{g}_{6 . N 8}$ & {$\left[X_{1}, X_{2}\right]=X_{3}+X_{5},\left[X_{1}, X_{3}\right]=X_{4},\left[X_{2}, X_{5}\right]=X_{6}$} \\
\hline $\mathfrak{g}_{6 . N 9}$ & {$\left[X_{1}, X_{2}\right]=X_{3},\left[X_{1}, X_{3}\right]=X_{4},\left[X_{1}, X_{5}\right]=X_{6},\left[X_{2}, X_{3}\right]=X_{5}$} \\
\hline $\mathfrak{g}_{6 . N 10}$ & {$\left[X_{1}, X_{2}\right]=X_{3},\left[X_{1}, X_{3}\right]=X_{5},\left[X_{1}, X_{4}\right]=X_{6}$,} \\
& {$\left[X_{2}, X_{3}\right]=-X_{6},\left[X_{2}, X_{4}\right]=X_{5}$} \\
\hline $\mathfrak{g}_{6 . N 11}$ & {$\left[X_{1}, X_{2}\right]=X_{3},\left[X_{1}, X_{3}\right]=X_{4},\left[X_{1}, X_{4}\right]=X_{5},\left[X_{2}, X_{3}\right]=X_{6}$} \\
\hline $\mathfrak{g}_{6 . N 12}$ & {$\left[X_{1}, X_{3}\right]=X_{4},\left[X_{1}, X_{4}\right]=X_{6},\left[X_{2}, X_{5}\right]=X_{6}$} \\
\hline $\mathfrak{g}_{6 . N 13}$ & {$\left[X_{1}, X_{2}\right]=X_{5},\left[X_{1}, X_{3}\right]=X_{4},\left[X_{1}, X_{4}\right]=X_{6},\left[X_{2}, X_{5}\right]=X_{6}$} \\
\hline $\mathfrak{g}_{6 . N 14}^{ \pm 1}$ & {$\left[X_{1}, X_{3}\right]=X_{4},\left[X_{1}, X_{4}\right]=X_{6},\left[X_{2}, X_{3}\right]=X_{5},\left[X_{2}, X_{5}\right]= \pm X_{6}$} \\
\hline $\mathfrak{g}_{6 . N 15}\left[X_{1}, X_{2}\right]=X_{3}+X_{5},\left[X_{1}, X_{3}\right]=X_{4},\left[X_{1}, X_{4}\right]=X_{6},\left[X_{2}, X_{5}\right]=X_{6}$ \\
\hline
\end{tabular}


Table 16: 6-dimensional indecomposable nilpotent algebras (continued)

\begin{tabular}{|c|c|}
\hline \hline & {$\left[X_{i}, X_{j}\right]$} \\
\hline \hline $\mathfrak{g}_{6 . N 16}$ & {$\left[X_{1}, X_{3}\right]=X_{4},\left[X_{1}, X_{4}\right]=X_{5},\left[X_{1}, X_{5}\right]=X_{6}$,} \\
& {$\left[X_{2}, X_{3}\right]=X_{5},\left[X_{2}, X_{4}\right]=X_{6}$} \\
\hline $\mathfrak{g}_{6 . N 17}$ & {$\left[X_{1}, X_{2}\right]=X_{3},\left[X_{1}, X_{3}\right]=X_{4},\left[X_{1}, X_{4}\right]=X_{6},\left[X_{2}, X_{5}\right]=X_{6}$} \\
\hline $\mathfrak{g}_{6 . N 18}^{ \pm 1}$ & {$\left[X_{1}, X_{2}\right]=X_{3},\left[X_{1}, X_{3}\right]=X_{4},\left[X_{1}, X_{4}\right]=X_{6}$,} \\
& {$\left[X_{2}, X_{3}\right]=X_{5},\left[X_{2}, X_{5}\right]= \pm X_{6}$} \\
\hline $\mathfrak{g}_{6 . N 19}$ & {$\left[X_{1}, X_{2}\right]=X_{3},\left[X_{1}, X_{3}\right]=X_{4},\left[X_{1}, X_{4}\right]=X_{5}$,} \\
& {$\left[X_{1}, X_{5}\right]=X_{6},\left[X_{2}, X_{3}\right]=X_{6}$} \\
\hline $\mathfrak{g}_{6 . N 20}$ & {$\left[X_{1}, X_{2}\right]=X_{3},\left[X_{1}, X_{3}\right]=X_{4},\left[X_{1}, X_{4}\right]=X_{5}$,} \\
& {$\left[X_{1}, X_{5}\right]=X_{6},\left[X_{2}, X_{3}\right]=X_{5},\left[X_{2}, X_{4}\right]=X_{6}$} \\
\hline $\mathfrak{g}_{6 . N 21}$ & {$\left[X_{1}, X_{2}\right]=X_{3},\left[X_{1}, X_{5}\right]=X_{6},\left[X_{2}, X_{3}\right]=X_{4}$,} \\
& {$\left[X_{2}, X_{4}\right]=X_{5},\left[X_{3}, X_{4}\right]=X_{6}$} \\
\hline $\mathfrak{g}_{6 . N 22}$ & {$\left[X_{1}, X_{2}\right]=X_{3},\left[X_{1}, X_{3}\right]=X_{5},\left[X_{1}, X_{5}\right]=X_{6}$,} \\
& {$\left[X_{2}, X_{3}\right]=X_{4},\left[X_{2}, X_{4}\right]=X_{5},\left[X_{3}, X_{4}\right]=X_{6}$} \\
\hline
\end{tabular}

Mubarakzjanov's list in [43] contains 99 classes of six-dimensional indecomposable almost nilpotent Lie algebras, namely $\mathfrak{g}_{6.1}, \ldots, \mathfrak{g}_{6.99}$.

As first remarked by Turkowski, there is one algebra missing. The complete (and partly corrected) list can be found in the article [10] of Campoamor-Stursberg ${ }^{13}$, where the missing algebra is denoted by $\mathfrak{g}_{6.92}^{*}$.

We list the unimodular among this 100 algebras in Tables 17 to 30 (where some minor misprints have been corrected). Note that there is no table with Lie algebras with nilradical $\mathfrak{g}_{5.6}$ since the only such algebra is not unimodular.

Table 17: 6-dimensional indecomposable unimodular almost abelian algebras

\begin{tabular}{|c|c|c|}
\hline \hline & {$\left[X_{i}, X_{j}\right]$} & cpl. solv. \\
\hline \hline $\mathfrak{g}_{6.1}^{a, b, c, d}$ & {$\left[X_{1}, X_{6}\right]=X_{1},\left[X_{2}, X_{6}\right]=a X_{2},\left[X_{3}, X_{6}\right]=b X_{3}$,} & yes \\
& {$\left[X_{4}, X_{6}\right]=c X_{4},\left[X_{5}, X_{6}\right]=d X_{5}$} & \\
& $0<|d| \leq|c| \leq|b| \leq|a| \leq 1, a+b+c+d=-1$ & \\
\hline
\end{tabular}

\footnotetext{
${ }^{13}$ The author wishes to express his gratitude to R. Campoamor-Stursberg for providing him with copies of [10] and [43].
} 
Table 18: 6-dimensional indecomposable unimodular almost abelian algebras (continued)

\begin{tabular}{|c|c|c|}
\hline & $\overline{[}\left[X_{i}, X_{j}\right]$ & c. s. \\
\hline $\mathfrak{g}_{6.2}^{a, c, d}$ & $\begin{aligned} {\left[X_{1}, X_{6}\right]=} & a X_{1},\left[X_{2}, X_{6}\right]=X_{1}+a X_{2},\left[X_{3}, X_{6}\right]=X_{3}, \\
& {\left[X_{4}, X_{6}\right]=c X_{4},\left[X_{5}, X_{6}\right]=d X_{5} } \\
& 0<|d| \leq|c| \leq 1,2 a+c+d=-1\end{aligned}$ & 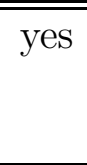 \\
\hline $\mathfrak{g}_{6.3}^{-\frac{d+1}{3}, d}$ & $\begin{array}{c}{\left[X_{1}, X_{6}\right]=-\frac{d+1}{3} X_{1},\left[X_{2}, X_{6}\right]=X_{1}-\frac{d+1}{3} X_{2}} \\
{\left[X_{3}, X_{6}\right]=X_{2}-\frac{d+1}{3} X_{3},\left[X_{4}, X_{6}\right]=X_{4},\left[X_{5}, X_{6}\right]=d X_{5}} \\
0<|d| \leq 1\end{array}$ & yes \\
\hline $\mathfrak{g}_{6.4}^{-\frac{1}{4}}$ & $\begin{array}{c}{\left[X_{1}, X_{6}\right]=-\frac{1}{4} X_{1},\left[X_{2}, X_{6}\right]=X_{1}-\frac{1}{4} X_{2}} \\
{\left[X_{3}, X_{6}\right]=X_{2}-\frac{1}{4} X_{3},\left[X_{4}, X_{6}\right]=X_{3}-\frac{1}{4} X_{4},\left[X_{5}, X_{6}\right]=X_{5}}\end{array}$ & yes \\
\hline $\mathfrak{g}_{6.6}^{a, b}$ & $\begin{array}{c}{\left[X_{1}, X_{6}\right]=X_{1},\left[X_{2}, X_{6}\right]=a X_{2},\left[X_{3}, X_{6}\right]=X_{2}+a X_{3}} \\
{\left[X_{4}, X_{6}\right]=b X_{4},\left[X_{5}, X_{6}\right]=X_{4}+b X_{5}, a \leq b, a+b=-\frac{1}{2}}\end{array}$ & yes \\
\hline $\mathfrak{g}_{6.7}^{a,-\frac{2}{3} a}$ & $\begin{array}{c}{\left[X_{1}, X_{6}\right]=a X_{1},\left[X_{2}, X_{6}\right]=X_{1}+a X_{2},\left[X_{3}, X_{6}\right]=X_{2}+a X_{3},} \\
{\left[X_{4}, X_{6}\right]=-\frac{2}{3} a X_{4},\left[X_{5}, X_{6}\right]=X_{4}-\frac{2}{3} a X_{5}, a \neq 0}\end{array}$ & yes \\
\hline $\mathfrak{g}_{6.8}^{a, b, c, p}$ & $\begin{array}{c}{\left[X_{1}, X_{6}\right]=a X_{1},\left[X_{2}, X_{6}\right]=b X_{2},\left[X_{3}, X_{6}\right]=c X_{3}} \\
{\left[X_{4}, X_{6}\right]=p X_{4}-X_{5},\left[X_{5}, X_{6}\right]=X_{4}+p X_{5}} \\
0<|c| \leq|b| \leq|a|, a+b+c+2 p=0\end{array}$ & no \\
\hline $\mathfrak{g}_{6.9}^{a, b, p}$ & $\begin{array}{c}{\left[X_{1}, X_{6}\right]=a X_{1},\left[X_{2}, X_{6}\right]=b X_{2},\left[X_{3}, X_{6}\right]=X_{2}+b X_{3},} \\
{\left[X_{4}, X_{6}\right]=p X_{4}-X_{5},\left[X_{5}, X_{6}\right]=X_{4}+p X_{5},} \\
a \neq 0, a+2 b+2 p=0\end{array}$ & no \\
\hline $\mathfrak{g}_{6.10}^{a,-\frac{3}{2} a}$ & $\begin{array}{c}{\left[X_{1}, X_{6}\right]=a X_{1},\left[X_{2}, X_{6}\right]=X_{1}+a X_{2},\left[X_{3}, X_{6}\right]=X_{2}+a X_{3},} \\
{\left[X_{4}, X_{6}\right]=-\frac{3}{2} a X_{4}-X_{5},\left[X_{5}, X_{6}\right]=X_{4}-\frac{3}{2} a X_{5}}\end{array}$ & no \\
\hline $\mathfrak{g}_{6.11}^{a, p, q, s}$ & $\begin{array}{c}{\left[X_{1}, X_{6}\right]=a X_{1},\left[X_{2}, X_{6}\right]=p X_{2}-X_{3},\left[X_{3}, X_{6}\right]=X_{2}+p X_{3},} \\
{\left[X_{4}, X_{6}\right]=q X_{4}-s X_{5},\left[X_{5}, X_{6}\right]=s X_{4}+q X_{5},} \\
a s \neq 0, a+2 p+2 q=0\end{array}$ & no \\
\hline $\mathfrak{g}_{6.12}^{-4 p, p}$ & $\begin{array}{c}{\left[X_{1}, X_{6}\right]=-4 p X_{1},\left[X_{2}, X_{6}\right]=p X_{2}-X_{3}} \\
{\left[X_{3}, X_{6}\right]=X_{2}+p X_{3},\left[X_{4}, X_{6}\right]=X_{2}+p X_{4}-X_{5}} \\
{\left[X_{5}, X_{6}\right]=X_{3}+X_{4}+p X_{5}, p \neq 0}\end{array}$ & no \\
\hline
\end{tabular}

Table 19: 6-dimensional indecomposable unimodular algebras with nilradical $\mathfrak{g}_{3.1} \oplus 2 \mathfrak{g}_{1}$

\begin{tabular}{|c|c|c|}
\hline \hline & {$\left[X_{i}, X_{j}\right]$} & cpl. solv. \\
\hline \hline $\mathfrak{g}_{6.13}^{a, b, h}$ & {$\left[X_{2}, X_{3}\right]=X_{1},\left[X_{1}, X_{6}\right]=(a+b) X_{1},\left[X_{2}, X_{6}\right]=a X_{2}$,} & yes \\
& {$\left[X_{3}, X_{6}\right]=b X_{3},\left[X_{4}, X_{6}\right]=X_{4},\left[X_{5}, X_{6}\right]=h X_{5}$,} & \\
& $a \neq 0,2 a+2 b+h=-1$ & \\
\hline $\mathfrak{g}_{6.14}^{a, b}$ & {$\left[X_{2}, X_{3}\right]=X_{1},\left[X_{1}, X_{6}\right]=(a+b) X_{1},\left[X_{2}, X_{6}\right]=a X_{2}$,} & yes \\
& {$\left[X_{3}, X_{6}\right]=b X_{3},\left[X_{4}, X_{6}\right]=X_{4},\left[X_{5}, X_{6}\right]=X_{1}+(a+b) X_{5}$,} & \\
& $a \neq 0, a+b=-\frac{1}{3}$ & \\
\hline
\end{tabular}


Table 20: 6-dimensional indecomposable unimodular algebras with nilradical $\mathfrak{g}_{3.1} \oplus 2 \mathfrak{g}_{1}$ (continued)

\begin{tabular}{|c|c|c|}
\hline & {$\left[X_{i}, X_{j}\right]$} & c. s. \\
\hline $\mathfrak{g}_{6.15}^{-1}$ & $\begin{array}{c}{\left[X_{2}, X_{3}\right]=X_{1},\left[X_{2}, X_{6}\right]=X_{2}+X_{4},} \\
{\left[X_{3}, X_{6}\right]=-X_{3}+X_{5},\left[X_{4}, X_{6}\right]=X_{4},\left[X_{5}, X_{6}\right]=-X_{5},}\end{array}$ & $\overline{\text { yes }}$ \\
\hline $\mathfrak{g}_{6.17}^{-\frac{1}{2}, 0}$ & $\begin{aligned} {\left[X_{2}, X_{3}\right]=} & X_{1},\left[X_{1}, X_{6}\right]=-\frac{1}{2} X_{1},\left[X_{2}, X_{6}\right]=-\frac{1}{2} X_{2} \\
& {\left[X_{3}, X_{6}\right]=X_{4},\left[X_{5}, X_{6}\right]=X_{5} }\end{aligned}$ & yes \\
\hline $\mathfrak{g}_{6.18}^{a,-2 a-3}$ & $\begin{array}{c}{\left[X_{2}, X_{3}\right]=X_{1},\left[X_{1}, X_{6}\right]=(1+a) X_{1},\left[X_{2}, X_{6}\right]=a X_{2}} \\
{\left[X_{3}, X_{6}\right]=X_{3}+X_{4},\left[X_{4}, X_{6}\right]=X_{4}} \\
{\left[X_{5}, X_{6}\right]=-(2 a+3) X_{5}, a \neq-\frac{3}{2}}\end{array}$ & yes \\
\hline $\mathfrak{g}_{6.19}^{-\frac{4}{3}}$ & $\begin{array}{c}{\left[X_{2}, X_{3}\right]=X_{1},\left[X_{1}, X_{6}\right]=-\frac{1}{3} X_{1},\left[X_{2}, X_{6}\right]=-\frac{4}{3} X_{2},} \\
{\left[X_{3}, X_{6}\right]=X_{3}+X_{4},\left[X_{4}, X_{6}\right]=X_{4},\left[X_{5}, X_{6}\right]=X_{1}-\frac{1}{3} X_{5}}\end{array}$ & yes \\
\hline$\frac{\mathfrak{g}_{6.20}^{-3}}{4}$ & $\begin{array}{c}{\left[X_{2}, X_{3}\right]=X_{1},\left[X_{1}, X_{6}\right]=X_{1},\left[X_{3}, X_{6}\right]=X_{3}+X_{4}} \\
{\left[X_{4}, X_{6}\right]=X_{1}+X_{4},\left[X_{5}, X_{6}\right]=-3 X_{5}}\end{array}$ & yes \\
\hline $\mathfrak{g}_{6.21}^{a}$ & $\begin{array}{c}{\left[X_{2}, X_{3}\right]=X_{1},\left[X_{1}, X_{6}\right]=2 a X_{1},\left[X_{2}, X_{6}\right]=a X_{2}+X_{3}} \\
{\left[X_{3}, X_{6}\right]=a X_{3},\left[X_{4}, X_{6}\right]=X_{4},\left[X_{5}, X_{6}\right]=-(4 a+1) X_{5}} \\
a \neq-\frac{1}{4}\end{array}$ & yes \\
\hline $\mathfrak{g}_{6.22}^{-\frac{1}{6}}$ & $\begin{array}{c}{\left[X_{2}, X_{3}\right]=X_{1},\left[X_{1}, X_{6}\right]=-\frac{1}{3} X_{1},\left[X_{2}, X_{6}\right]=-\frac{1}{6} X_{2}+X_{3},} \\
{\left[X_{3}, X_{6}\right]=-\frac{1}{6} X_{3},\left[X_{4}, X_{6}\right]=X_{4},\left[X_{5}, X_{6}\right]=X_{1}-\frac{1}{3} X_{5}}\end{array}$ & yes \\
\hline $\mathfrak{g}_{6.23}^{a,-7 a, \varepsilon}$ & $\begin{array}{c}{\left[X_{2}, X_{3}\right]=X_{1},\left[X_{1}, X_{6}\right]=2 a X_{1},\left[X_{2}, X_{6}\right]=a X_{2}+X_{3}} \\
{\left[X_{3}, X_{6}\right]=a X_{3}+X_{4},\left[X_{4}, X_{6}\right]=a X_{4}} \\
{\left[X_{5}, X_{6}\right]=\varepsilon X_{1}-5 a X_{5}, \varepsilon a=0}\end{array}$ & yes \\
\hline $\mathfrak{g}_{6.25}^{b,-1-b}$ & $\begin{array}{c}{\left[X_{2}, X_{3}\right]=X_{1},\left[X_{1}, X_{6}\right]=-b X_{1}} \\
{\left[X_{2}, X_{6}\right]=X_{2},\left[X_{3}, X_{6}\right]=-(1+b) X_{3}} \\
{\left[X_{4}, X_{6}\right]=b X_{4}+X_{5},\left[X_{5}, X_{6}\right]=b X_{5}}\end{array}$ & yes \\
\hline $\mathfrak{g}_{6.26}^{-1}$ & $\begin{array}{c}{\left[X_{2}, X_{3}\right]=X_{1},\left[X_{2}, X_{6}\right]=X_{2},\left[X_{3}, X_{6}\right]=-X_{3}} \\
{\left[X_{4}, X_{6}\right]=X_{5},\left[X_{5}, X_{6}\right]=X_{1}}\end{array}$ & yes \\
\hline $\mathfrak{g}_{6.27}^{-26, b}$ & $\begin{array}{c}{\left[X_{2}, X_{3}\right]=X_{1},\left[X_{1}, X_{6}\right]=-b X_{1},\left[X_{2}, X_{6}\right]=-2 b X_{2}} \\
{\left[X_{3}, X_{6}\right]=b X_{3}+X_{4},\left[X_{4}, X_{6}\right]=b X_{4}+X_{5}} \\
{\left[X_{5}, X_{6}\right]=b X_{5}, b \neq 0}\end{array}$ & yes \\
\hline $\mathfrak{g}_{6.28}^{-2}$ & $\begin{array}{c}{\left[X_{2}, X_{3}\right]=X_{1},\left[X_{1}, X_{6}\right]=2 X_{1},\left[X_{2}, X_{6}\right]=X_{2}+X_{3},} \\
{\left[X_{3}, X_{6}\right]=X_{3},\left[X_{4}, X_{6}\right]=-2 X_{4}+X_{5},\left[X_{5}, X_{6}\right]=-2 X_{5}}\end{array}$ & yes \\
\hline $\mathfrak{g}_{6.29}^{-2 b, b, \varepsilon}$ & $\begin{array}{c}{\left[X_{2}, X_{3}\right]=X_{1},\left[X_{1}, X_{6}\right]=-b X_{1},\left[X_{2}, X_{6}\right]=-2 b X_{2}} \\
{\left[X_{3}, X_{6}\right]=b X_{3}+X_{4},\left[X_{4}, X_{6}\right]=b X_{4}+X_{5}} \\
{\left[X_{5}, X_{6}\right]=\varepsilon X_{1}+b X_{5}, \varepsilon b=0(?)}\end{array}$ & yes \\
\hline $\mathfrak{g}_{6.32}^{a,-6 a-h, h, \varepsilon}$ & $\begin{array}{c}{\left[X_{2}, X_{3}\right]=X_{1},\left[X_{1}, X_{6}\right]=2 a X_{1},\left[X_{2}, X_{6}\right]=a X_{2}+X_{3},} \\
{\left[X_{3}, X_{6}\right]=-X_{2}+a X_{3},\left[X_{4}, X_{6}\right]=\varepsilon X_{1}+(2 a+h) X_{4},} \\
{\left[X_{5}, X_{6}\right]=-(6 a+h) X_{5}, a>-\frac{1}{4} h, \varepsilon h=0}\end{array}$ & no \\
\hline
\end{tabular}


Table 21: 6-dimensional indecomposable unimodular algebras with nilradical $\mathfrak{g}_{3.1} \oplus 2 \mathfrak{g}_{1}$ (continued)

\begin{tabular}{|c|c|c|}
\hline \hline & {$\left[X_{i}, X_{j}\right]$} & c. s. \\
\hline \hline $\mathfrak{g}_{6.33}^{a,-6 a}$ & {$\left[X_{2}, X_{3}\right]=X_{1},\left[X_{1}, X_{6}\right]=2 a X_{1},\left[X_{2}, X_{6}\right]=a X_{2}+X_{3}$,} & no \\
& {$\left[X_{3}, X_{6}\right]=-X_{2}+a X_{3},\left[X_{4}, X_{6}\right]=-6 a X_{4}$,} & \\
& {$\left[X_{5}, X_{6}\right]=X_{1}+2 a X_{5}, a \geq 0$} & \\
\hline $\mathfrak{g}_{6.34}^{a,-4 a, \varepsilon}$ & {$\left[X_{2}, X_{3}\right]=X_{1},\left[X_{1}, X_{6}\right]=2 a X_{1},\left[X_{2}, X_{6}\right]=a X_{2}+X_{3}$,} & no \\
& {$\left[X_{3}, X_{6}\right]=-X_{2}+a X_{3},\left[X_{4}, X_{6}\right]=-2 a X_{4}$,} & \\
& {$\left[X_{5}, X_{6}\right]=\varepsilon X_{1}-2 a X_{5}, \varepsilon a=0$} & \\
\hline $\mathfrak{g}_{6.35}^{a, b, c}$ & {$\left[X_{2}, X_{3}\right]=X_{1},\left[X_{1}, X_{6}\right]=(a+b) X_{1},\left[X_{2}, X_{6}\right]=a X_{2}$,} & no \\
& {$\left[X_{3}, X_{6}\right]=b X_{3},\left[X_{4}, X_{6}\right]=c X_{4}+X_{5}$,} & \\
& {$\left[X_{5}, X_{6}\right]=-X_{4}+c X_{5}, a+b+c=0, a^{2}+b^{2} \neq 0$} & \\
\hline $\mathfrak{g}_{6.36}^{a,-2 a}$ & {$\left[X_{2}, X_{3}\right]=X_{1},\left[X_{1}, X_{6}\right]=2 a X_{1},\left[X_{2}, X_{6}\right]=a X_{2}+X_{3}$,} & no \\
& {$\left[X_{3}, X_{6}\right]=a X_{3},\left[X_{4}, X_{6}\right]=-2 a X_{4}+X_{5}$,} & \\
& {$\left[X_{5}, X_{6}\right]=-X_{4}-2 a X_{5}$} & no \\
\hline $\mathfrak{g}_{6.37}^{-a,-2 a, s}$ & {$\left[X_{2}, X_{3}\right]=X_{1},\left[X_{1}, X_{6}\right]=2 a X_{1},\left[X_{2}, X_{6}\right]=a X_{2}+X_{3}$,} & no \\
& {$\left[X_{3}, X_{6}\right]=-X_{2}+a X_{3},\left[X_{4}, X_{6}\right]=-2 a X_{4}+s X_{5}$,} & \\
& {$\left[X_{5}, X_{6}\right]=-s X_{4}-2 a X_{5}, s \neq 0$} & \\
\hline $\mathfrak{g}_{6.38}^{0}$ & {$\left[X_{2}, X_{3}\right]=X_{1},\left[X_{2}, X_{6}\right]=X_{3}+X_{4}$,} & \\
& {$\left[X_{3}, X_{6}\right]=-X_{2}+X_{5},\left[X_{4}, X_{6}\right]=X_{5},\left[X_{5}, X_{6}\right]=-X_{4}$} & \\
\hline
\end{tabular}

Table 22: 6-dimensional indecomposable unimodular algebras with nilradical $\mathfrak{g}_{4.1} \oplus \mathfrak{g}_{1}$

\begin{tabular}{|c|c|c|}
\hline \hline & {$\left[X_{i}, X_{j}\right]$} & c. s. \\
\hline \hline $\mathfrak{g}_{6.39}^{-4-3 h, h}$ & {$\left[X_{1}, X_{5}\right]=X_{2},\left[X_{4}, X_{5}\right]=X_{1},\left[X_{1}, X_{6}\right]=(1+h) X_{1}$,} & yes \\
& {$\left[X_{2}, X_{6}\right]=(2+h) X_{2},\left[X_{3}, X_{6}\right]=-(4+3 h) X_{3}$,} & \\
& {$\left[X_{4}, X_{6}\right]=h X_{4},\left[X_{5}, X_{6}\right]=X_{5}, h \neq-\frac{4}{3}$} & \\
\hline $\mathfrak{g}_{6.40}^{-\frac{3}{2}}$ & {$\left[X_{1}, X_{5}\right]=X_{2},\left[X_{4}, X_{5}\right]=X_{1}$} & yes \\
& {$\left[X_{1}, X_{6}\right]=-\frac{1}{2} X_{1},\left[X_{2}, X_{6}\right]=\frac{1}{2} X_{2}$,} & \\
& {$\left[X_{3}, X_{6}\right]=X_{2}+\frac{1}{2} X_{3},\left[X_{4}, X_{6}\right]=-\frac{3}{2} X_{4},\left[X_{5}, X_{6}\right]=X_{5}$} & \\
\hline $\mathfrak{g}_{6.41}^{-1}$ & {$\left[X_{1}, X_{5}\right]=X_{2},\left[X_{4}, X_{5}\right]=X_{1}$} & yes \\
& {$\left[X_{2}, X_{6}\right]=X_{2},\left[X_{3}, X_{6}\right]=-X_{3}$,} & \\
& {$\left[X_{4}, X_{6}\right]=X_{3}-X_{4},\left[X_{5}, X_{6}\right]=X_{5}$} & \\
\hline
\end{tabular}


Table 23: 6-dimensional indecomposable unimodular algebras with nilradical $\mathfrak{g}_{4.1} \oplus \mathfrak{g}_{1}$ (continued)

\begin{tabular}{|c|c|c|}
\hline \hline & {$\left[X_{i}, X_{j}\right]$} & c. s. \\
\hline \hline $\mathfrak{g}_{6.42}^{-\frac{5}{3}}$ & {$\left[X_{1}, X_{5}\right]=X_{2},\left[X_{4}, X_{5}\right]=X_{1}$,} & yes \\
& {$\left[X_{1}, X_{6}\right]=-\frac{2}{3} X_{1},\left[X_{2}, X_{6}\right]=\frac{1}{3} X_{2},\left[X_{3}, X_{6}\right]=X_{3}$} & \\
& {$\left[X_{4}, X_{6}\right]=-\frac{5}{3} X_{4},\left[X_{5}, X_{6}\right]=X_{3}+X_{5}$} & \\
\hline $\mathfrak{g}_{6.44}^{-7}$ & {$\left[X_{1}, X_{5}\right]=X_{2},\left[X_{4}, X_{5}\right]=X_{1}$,} & yes \\
& {$\left[X_{1}, X_{6}\right]=2 X_{1},\left[X_{2}, X_{6}\right]=3 X_{2},\left[X_{3}, X_{6}\right]=-7 X_{3}$} & \\
& {$\left[X_{4}, X_{6}\right]=X_{4},\left[X_{5}, X_{6}\right]=X_{4}+X_{5}$} & \\
\hline $\mathfrak{g}_{6.47}^{-3, \varepsilon}$ & {$\left[X_{1}, X_{5}\right]=X_{2},\left[X_{4}, X_{5}\right]=X_{1}$,} & yes \\
& {$\left[X_{1}, X_{6}\right]=X_{1},\left[X_{2}, X_{6}\right]=X_{2},\left[X_{3}, X_{6}\right]=-3 X_{3}$} & \\
& {$\left[X_{4}, X_{6}\right]=\varepsilon X_{2}+X_{4}, \varepsilon \in\{0, \pm 1\}$} & \\
\hline
\end{tabular}

Table 24: 6-dimensional indecomposable unimodular algebras with nilradical $\mathfrak{g}_{5.1}$

\begin{tabular}{|c|c|c|}
\hline & {$\left[X_{i}, X_{j}\right]$} & c. s. \\
\hline$\overline{\mathfrak{g}_{6.54}^{2(1+l), l}}$ & $\begin{array}{c}{\left[X_{3}, X_{5}\right]=X_{1},\left[X_{4}, X_{5}\right]=X_{2}} \\
{\left[X_{1}, X_{6}\right]=X_{1},\left[X_{2}, X_{6}\right]=l X_{2},\left[X_{3}, X_{6}\right]=(-1-2 l) X_{3}} \\
{\left[X_{4}, X_{6}\right]=(-2-l) X_{4},\left[X_{5}, X_{6}\right]=2(1+l) X_{5}}\end{array}$ & yes \\
\hline $\mathfrak{g}_{6.55}^{-4}$ & $\begin{array}{c}{\left[X_{3}, X_{5}\right]=X_{1},\left[X_{4}, X_{5}\right]=X_{2}} \\
{\left[X_{1}, X_{6}\right]=X_{1},\left[X_{2}, X_{6}\right]=-3 X_{2},\left[X_{3}, X_{6}\right]=4 X_{3}} \\
{\left[X_{4}, X_{6}\right]=X_{1}+X_{4},\left[X_{5}, X_{6}\right]=-3 X_{5}}\end{array}$ & yes \\
\hline $\mathfrak{g}_{6.56}^{\frac{4}{3}}$ & $\begin{array}{c}{\left[X_{3}, X_{5}\right]=X_{1},\left[X_{4}, X_{5}\right]=X_{2}} \\
{\left[X_{1}, X_{6}\right]=X_{1},\left[X_{2}, X_{6}\right]=-\frac{1}{3} X_{2},\left[X_{3}, X_{6}\right]=X_{2}-\frac{1}{3} X_{3}} \\
{\left[X_{4}, X_{6}\right]=-\frac{5}{3} X_{4},\left[X_{5}, X_{6}\right]=\frac{4}{3} X_{5}}\end{array}$ & yes \\
\hline $\mathfrak{g}_{6.57}^{-\frac{2}{3}}$ & $\begin{array}{c}{\left[X_{3}, X_{5}\right]=X_{1},\left[X_{4}, X_{5}\right]=X_{2}} \\
{\left[X_{1}, X_{6}\right]=X_{1},\left[X_{2}, X_{6}\right]=-\frac{4}{3} X_{2},\left[X_{3}, X_{6}\right]=\frac{5}{3} X_{3}} \\
{\left[X_{4}, X_{6}\right]=-\frac{2}{3} X_{4},\left[X_{5}, X_{6}\right]=X_{4}-\frac{2}{3} X_{5}}\end{array}$ & yes \\
\hline $\mathfrak{g}_{6.61}^{-\frac{3}{4}}$ & $\begin{array}{c}{\left[X_{3}, X_{5}\right]=X_{1},\left[X_{4}, X_{5}\right]=X_{2},} \\
{\left[X_{1}, X_{6}\right]=2 X_{1},\left[X_{2}, X_{6}\right]=-\frac{3}{2} X_{2},\left[X_{3}, X_{6}\right]=X_{3}} \\
{\left[X_{4}, X_{6}\right]=-\frac{5}{2} X_{4},\left[X_{5}, X_{6}\right]=X_{3}+X_{5}}\end{array}$ & yes \\
\hline $\mathfrak{g}_{6.63}^{-1}$ & $\begin{array}{c}{\left[X_{3}, X_{5}\right]=X_{1},\left[X_{4}, X_{5}\right]=X_{2}} \\
{\left[X_{1}, X_{6}\right]=X_{1},\left[X_{2}, X_{6}\right]=-X_{2},\left[X_{3}, X_{6}\right]=X_{3}} \\
{\left[X_{4}, X_{6}\right]=X_{2}-X_{4}}\end{array}$ & yes \\
\hline $\mathfrak{g}_{6.65}^{4 l, l}$ & $\begin{array}{c}{\left[X_{3}, X_{5}\right]=X_{1},\left[X_{4}, X_{5}\right]=X_{2}} \\
{\left[X_{1}, X_{6}\right]=l X_{1}+X_{2},\left[X_{2}, X_{6}\right]=l X_{2},\left[X_{3}, X_{6}\right]=-3 l X_{3}+X_{4}} \\
{\left[X_{4}, X_{6}\right]=-3 l X_{4},\left[X_{5}, X_{6}\right]=4 l X_{5}}\end{array}$ & yes \\
\hline
\end{tabular}


Table 25: 6-dimensional indecomposable unimodular algebras with nilradical $\mathfrak{g}_{5.1}$ (continued)

\begin{tabular}{|c|c|c|}
\hline \hline & {$\left[X_{i}, X_{j}\right]$} & cpl. solv. \\
\hline \hline $\mathfrak{g}_{6.70}^{4 p, p}$ & {$\left[X_{3}, X_{5}\right]=X_{1},\left[X_{4}, X_{5}\right]=X_{2}$,} & no \\
& {$\left[X_{1}, X_{6}\right]=p X_{1}+X_{2},\left[X_{2}, X_{6}\right]=-X_{1}+p X_{2}$,} & \\
& {$\left[X_{3}, X_{6}\right]=-3 p X_{3}+X_{4},\left[X_{4}, X_{6}\right]=-X_{3}-3 p X_{4}$,} & \\
& {$\left[X_{5}, X_{6}\right]=4 p X_{5}$} & \\
\hline
\end{tabular}

Table 26: 6-dimensional indecomposable unimodular algebras with nilradical $\mathfrak{g}_{5.2}$

\begin{tabular}{|c|c|c|}
\hline \hline & {$\left[X_{i}, X_{j}\right]$} & cpl. solv. \\
\hline \hline $\mathfrak{g}_{6.71}^{-\frac{7}{4}}$ & {$\left[X_{2}, X_{5}\right]=X_{1},\left[X_{3}, X_{5}\right]=X_{2},\left[X_{4}, X_{5}\right]=X_{3}$,} & yes \\
& {$\left[X_{1}, X_{6}\right]=\frac{5}{4} X_{1},\left[X_{2}, X_{6}\right]=\frac{1}{4} X_{2},\left[X_{3}, X_{6}\right]=-\frac{3}{4} X_{3}$} & \\
& {$\left[X_{4}, X_{6}\right]=-\frac{7}{4} X_{4},\left[X_{5}, X_{6}\right]=X_{5}$} & \\
\hline
\end{tabular}

Table 27: 6-dimensional indecomposable unimodular algebras with nilradical $\mathfrak{g}_{5.3}$

\begin{tabular}{|c|c|c|}
\hline \hline & {$\left[X_{i}, X_{j}\right]$} & cpl. solv. \\
\hline \hline $\mathfrak{g}_{6.76}^{-1}$ & {$\left[X_{2}, X_{4}\right]=X_{3},\left[X_{2}, X_{5}\right]=X_{1},\left[X_{4}, X_{5}\right]=X_{2}$} & yes \\
& {$\left[X_{1}, X_{6}\right]=-X_{1},\left[X_{3}, X_{6}\right]=X_{3}$,} & \\
& {$\left[X_{4}, X_{6}\right]=X_{4},\left[X_{5}, X_{6}\right]=-X_{5}$} & \\
\hline $\mathfrak{g}_{6.78}$ & {$\left[X_{2}, X_{4}\right]=X_{3},\left[X_{2}, X_{5}\right]=X_{1},\left[X_{4}, X_{5}\right]=X_{2}$} & yes \\
& {$\left[X_{1}, X_{6}\right]=-X_{1},\left[X_{3}, X_{6}\right]=X_{3}$,} & \\
& {$\left[X_{4}, X_{6}\right]=X_{3}+X_{4},\left[X_{5}, X_{6}\right]=-X_{5}$} & \\
\hline
\end{tabular}

Table 28: 6-dimensional indecomposable unimodular algebras with nilradical $\mathfrak{g}_{5.4}$

\begin{tabular}{|c|c|c|}
\hline \hline & {$\left[X_{i}, X_{j}\right]$} & cpl. solv. \\
\hline \hline $\mathfrak{g}_{6.83}^{0, l}$ & {$\left[X_{2}, X_{4}\right]=X_{1},\left[X_{3}, X_{5}\right]=X_{1}$,} & yes \\
& {$\left[X_{2}, X_{6}\right]=l X_{2},\left[X_{3}, X_{6}\right]=l X_{3}$,} & \\
& {$\left[X_{4}, X_{6}\right]=-l X_{4},\left[X_{5}, X_{6}\right]=-X_{4}-l X_{5}$} & \\
\hline $\mathfrak{g}_{6.84}$ & {$\left[X_{2}, X_{4}\right]=X_{1},\left[X_{3}, X_{5}\right]=X_{1}$,} & yes \\
& {$\left[X_{2}, X_{6}\right]=X_{2},\left[X_{4}, X_{6}\right]=-X_{4},\left[X_{5}, X_{6}\right]=X_{3}$} & \\
\hline
\end{tabular}


Table 29: 6-dimensional indecomposable unimodular algebras with nilradical $\mathfrak{g}_{5.4}$ (continued)

\begin{tabular}{|c|c|c|}
\hline & {$\left[X_{i}, X_{j}\right]$} & cpl. solv. \\
\hline $\mathfrak{g}_{6.88}^{0, \mu_{0}, \nu_{0}}$ & $\begin{array}{c}{\left[X_{2}, X_{4}\right]=X_{1},\left[X_{3}, X_{5}\right]=X_{1},} \\
{\left[X_{2}, X_{6}\right]=\mu_{0} X_{2}+\nu_{0} X_{3},\left[X_{3}, X_{6}\right]=-\nu_{0} X_{2}+\mu_{0} X_{3},} \\
{\left[X_{4}, X_{6}\right]=-\mu_{0} X_{4}+\nu_{0} X_{5},\left[X_{5}, X_{6}\right]=-\nu_{0} X_{4}-\mu_{0} X_{5}}\end{array}$ & $\begin{array}{c}\text { cpl. solv. } \\
\qquad \begin{array}{l}\mathbb{1} \\
\nu_{0}=0\end{array}\end{array}$ \\
\hline $\begin{array}{l}\mathfrak{g}_{6.89}^{0, \nu_{0}, s} \\
\end{array}$ & $\begin{array}{c}{\left[X_{2}, X_{4}\right]=X_{1},\left[X_{3}, X_{5}\right]=X_{1}} \\
{\left[X_{2}, X_{6}\right]=s X_{2},\left[X_{3}, X_{6}\right]=\nu_{0} X_{5}} \\
{\left[X_{4}, X_{6}\right]=-s X_{4},\left[X_{5}, X_{6}\right]=-\nu_{0} X_{3}}\end{array}$ & $\begin{array}{l}\text { cpl. solv. } \\
\quad \Uparrow \\
\quad \nu_{0}=0\end{array}$ \\
\hline $\mathfrak{g}_{6.90}^{0, \nu_{0}}$ & $\begin{array}{c}{\left[X_{2}, X_{4}\right]=X_{1},\left[X_{3}, X_{5}\right]=X_{1}} \\
{\left[X_{2}, X_{6}\right]=X_{4},\left[X_{3}, X_{6}\right]=\nu_{0} X_{5}} \\
{\left[X_{4}, X_{6}\right]=X_{2},\left[X_{5}, X_{6}\right]=-\nu_{0} X_{3}, \nu_{0} \neq 1}\end{array}$ & $\begin{array}{l}\text { cpl. solv. } \\
\qquad \begin{array}{l}\mathbb{1} \\
\nu_{0}=0\end{array}\end{array}$ \\
\hline $\mathfrak{g}_{6.91}$ & $\begin{array}{c}{\left[X_{2}, X_{4}\right]=X_{1},\left[X_{3}, X_{5}\right]=X_{1}} \\
{\left[X_{2}, X_{6}\right]=X_{4},\left[X_{3}, X_{6}\right]=X_{5}} \\
{\left[X_{4}, X_{6}\right]=X_{2},\left[X_{5}, X_{6}\right]=-X_{3}}\end{array}$ & no \\
\hline $\mathfrak{g}_{6.92}^{0, \mu_{0}, \nu_{0}}$ & $\begin{array}{c}{\left[X_{2}, X_{4}\right]=X_{1},\left[X_{3}, X_{5}\right]=X_{1}} \\
{\left[X_{2}, X_{6}\right]=\nu_{0} X_{3},\left[X_{3}, X_{6}\right]=-\mu_{0} X_{2}} \\
{\left[X_{4}, X_{6}\right]=\mu_{0} X_{5},\left[X_{5}, X_{6}\right]=-\nu_{0} X_{4}}\end{array}$ & no \\
\hline $\mathfrak{g}_{6.92^{*}}^{0}$ & $\begin{array}{c}{\left[X_{2}, X_{4}\right]=X_{1},\left[X_{3}, X_{5}\right]=X_{1}} \\
{\left[X_{2}, X_{6}\right]=X_{4},\left[X_{3}, X_{6}\right]=X_{5}} \\
{\left[X_{4}, X_{6}\right]=-X_{2},\left[X_{5}, X_{6}\right]=-X_{3}}\end{array}$ & no \\
\hline $\mathfrak{g}_{6.93}^{0, \nu_{0}}$ & $\begin{array}{c}{\left[X_{2}, X_{4}\right]=X_{1},\left[X_{3}, X_{5}\right]=X_{1}} \\
{\left[X_{2}, X_{6}\right]=X_{4}+\nu_{0} X_{5},\left[X_{3}, X_{6}\right]=\nu_{0} X_{4}} \\
{\left[X_{4}, X_{6}\right]=X_{2}-\nu_{0} X_{3},\left[X_{5}, X_{6}\right]=-\nu_{0} X_{2}}\end{array}$ & $\begin{array}{l}\text { cpl. solv. } \\
\text { \ } \\
\left|\nu_{0}\right| \leq \frac{1}{2}\end{array}$ \\
\hline
\end{tabular}

Table 30: 6-dimensional indecomposable unimodular algebras with nilradical $\mathfrak{g}_{5.5}$

\begin{tabular}{|c|c|c|}
\hline \hline & {$\left[X_{i}, X_{j}\right]$} & cpl. solv. \\
\hline \hline $\mathfrak{g}_{6.94}^{-2}$ & {$\left[X_{3}, X_{4}\right]=X_{1},\left[X_{2}, X_{5}\right]=X_{1},\left[X_{3}, X_{5}\right]=X_{2}$} & yes \\
& {$\left[X_{2}, X_{6}\right]=-X_{2},\left[X_{3}, X_{6}\right]=-2 X_{3}$,} & \\
& {$\left[X_{4}, X_{6}\right]=2 X_{4},\left[X_{5}, X_{6}\right]=X_{5}$} & \\
\hline
\end{tabular}

The six-dimensional solvable Lie algebras with four-dimensional nilradical were classified by Turkowski in [58. We list the unimodular among them in Tables 31 - 33. Note that there is no table with Lie algebras with nilradical $\mathfrak{g}_{4.1}$ since the only such algebra is not unimodular.

The equations for the twenty-fifth algebra in Turkowoski's list contain a minor misprint that we have corrected here. 
Table 31: 6-dimensional indecomposable unimodular algebras with nilradical $4 \mathfrak{g}_{1}$

\begin{tabular}{|c|c|c|}
\hline & {$\left[X_{i}, X_{j}\right]$} & C. S. \\
\hline $\begin{array}{l}\mathfrak{g}_{6.101}^{a, b, c, d} \\
\end{array}$ & $\begin{array}{c}{\left[X_{5}, X_{1}\right]=a X_{1},\left[X_{5}, X_{2}\right]=c X_{2},\left[X_{5}, X_{4}\right]=X_{4}} \\
{\left[X_{6}, X_{1}\right]=b X_{1},\left[X_{6}, X_{2}\right]=d X_{2},\left[X_{6}, X_{3}\right]=X_{3}} \\
a+c=-1, b+d=-1, a b \neq 0, c^{2}+d^{2} \neq 0\end{array}$ & yes \\
\hline $\mathfrak{g}_{6.102}^{-1, b,-2-b}$ & $\begin{array}{c}{\left[X_{5}, X_{1}\right]=-X_{1},\left[X_{5}, X_{2}\right]=X_{2},\left[X_{5}, X_{3}\right]=X_{4}} \\
{\left[X_{6}, X_{1}\right]=b X_{1},\left[X_{6}, X_{2}\right]=(-2-b) X_{2}} \\
{\left[X_{6}, X_{3}\right]=X_{3},\left[X_{6}, X_{4}\right]=X_{4}}\end{array}$ & yes \\
\hline $\mathfrak{g}_{6.105}^{-2,-1}$ & $\begin{array}{c}{\left[X_{5}, X_{1}\right]=-2 X_{1},\left[X_{5}, X_{3}\right]=X_{3}+X_{4}} \\
{\left[X_{5}, X_{4}\right]=X_{4},\left[X_{6}, X_{1}\right]=-X_{1},\left[X_{6}, X_{2}\right]=X_{2}}\end{array}$ & yes \\
\hline $\mathfrak{g}_{6.107}^{-1, b, 0}$ & $\begin{array}{c}{\left[X_{5}, X_{1}\right]=-X_{1},\left[X_{5}, X_{2}\right]=-X_{2},\left[X_{5}, X_{3}\right]=X_{3}+X_{4}} \\
{\left[X_{5}, X_{4}\right]=X_{4},\left[X_{6}, X_{1}\right]=X_{2},\left[X_{6}, X_{2}\right]=-X_{1}}\end{array}$ & no \\
\hline $\mathfrak{g}_{6.113}^{a, b,-a, d}$ & $\begin{array}{c}{\left[X_{5}, X_{1}\right]=a X_{1},\left[X_{5}, X_{2}\right]=-a X_{2},\left[X_{5}, X_{3}\right]=X_{4}} \\
{\left[X_{6}, X_{1}\right]=b X_{1},\left[X_{6}, X_{2}\right]=d X_{2},\left[X_{6}, X_{3}\right]=X_{3}} \\
{\left[X_{6}, X_{4}\right]=X_{4}, a^{2}+b^{2} \neq 0, a^{2}+d^{2} \neq 0, b+d=-2}\end{array}$ & no \\
\hline $\mathfrak{g}_{6.114}^{a,-1,-\frac{u}{2}}$ & $\begin{array}{c}{\left[X_{5}, X_{1}\right]=a X_{1},\left[X_{5}, X_{3}\right]=-\frac{a}{2} X_{3}+X_{4}} \\
{\left[X_{5}, X_{4}\right]=-X_{3}+\frac{a}{2} X_{4},\left[X_{6}, X_{1}\right]=-X_{1}} \\
{\left[X_{6}, X_{2}\right]=X_{2}, a \neq 0}\end{array}$ & no \\
\hline $\mathfrak{g}_{6.115}^{-1, b, c,-c}$ & $\begin{array}{c}{\left[X_{5}, X_{1}\right]=X_{1},\left[X_{5}, X_{2}\right]=X_{2}} \\
{\left[X_{5}, X_{3}\right]=-X_{3}+b X_{4},\left[X_{5}, X_{4}\right]=-b X_{3}-X_{4}} \\
{\left[X_{6}, X_{1}\right]=c X_{1}+X_{2},\left[X_{6}, X_{2}\right]=-X_{1}+c X_{2}} \\
{\left[X_{6}, X_{3}\right]=-c X_{3},\left[X_{6}, X_{4}\right]=-c X_{4}, b \neq 0}\end{array}$ & no \\
\hline $\mathfrak{g}_{6.116}^{0,-1}$ & $\begin{array}{c}{\left[X_{5}, X_{1}\right]=X_{2},\left[X_{5}, X_{3}\right]=X_{4},\left[X_{5}, X_{4}\right]=-X_{3}} \\
{\left[X_{6}, X_{1}\right]=X_{1},\left[X_{6}, X_{2}\right]=X_{2}} \\
{\left[X_{6}, X_{3}\right]=-X_{3},\left[X_{6}, X_{4}\right]=-X_{4}}\end{array}$ & no \\
\hline $\mathfrak{g}_{6.118}^{0, b,-1}$ & $\begin{array}{c}{\left[X_{5}, X_{1}\right]=X_{2},\left[X_{5}, X_{2}\right]=-X_{1},\left[X_{5}, X_{3}\right]=b X_{4}} \\
{\left[X_{5}, X_{4}\right]=-b X_{3},\left[X_{6}, X_{1}\right]=X_{1},\left[X_{6}, X_{2}\right]=X_{2}} \\
{\left[X_{6}, X_{3}\right]=-X_{3},\left[X_{6}, X_{4}\right]=-X_{4}, b \neq 0}\end{array}$ & no \\
\hline $\mathfrak{g}_{6.120}^{-1,-1}$ & $\begin{array}{c}{\left[X_{5}, X_{2}\right]=-X_{2},\left[X_{5}, X_{4}\right]=X_{4},\left[X_{5}, X_{6}\right]=X_{1}} \\
{\left[X_{6}, X_{2}\right]=-X_{1},\left[X_{6}, X_{3}\right]=X_{3}}\end{array}$ & yes \\
\hline $\mathfrak{g}_{6.125}^{0,-2}$ & $\begin{array}{l}{\left[X_{5}, X_{3}\right]=X_{4},\left[X_{5}, X_{4}\right]=-X_{3},\left[X_{5}, X_{6}\right]=X_{1}} \\
{\left[X_{6}, X_{2}\right]=-2 X_{2},\left[X_{6}, X_{3}\right]=X_{3},\left[X_{6}, X_{4}\right]=X_{4}}\end{array}$ & no \\
\hline
\end{tabular}

Table 32: 6-dimensional indecomposable unimodular algebras with nilradical $\mathfrak{g}_{3.1} \oplus \mathfrak{g}_{1}$

\begin{tabular}{|c|c|c|}
\hline \hline & {$\left[X_{i}, X_{j}\right]$} & c. s. \\
\hline \hline $\mathfrak{g}_{6.129}^{-2,-2}$ & {$\left[X_{2}, X_{3}\right]=X_{1},\left[X_{5}, X_{1}\right]=X_{1},\left[X_{5}, X_{2}\right]=X_{2}$,} & yes \\
& {$\left[X_{5}, X_{4}\right]=-2 X_{4},\left[X_{6}, X_{1}\right]=X_{1}$,} & \\
& {$\left[X_{6}, X_{3}\right]=X_{3},\left[X_{6}, X_{4}\right]=-2 X_{4}$} & \\
\hline
\end{tabular}


Table 33: 6-dimensional indecomposable unimodular algebras with nilradical $\mathfrak{g}_{3.1} \oplus \mathfrak{g}_{1}$ (continued)

\begin{tabular}{|c|c|c|}
\hline \hline & {$\left[X_{i}, X_{j}\right]$} & c. s. \\
\hline \hline $\mathfrak{g}_{6.135}^{0,-4}$ & {$\left[X_{2}, X_{3}\right]=X_{1},\left[X_{5}, X_{2}\right]=X_{3},\left[X_{5}, X_{3}\right]=-X_{1}$,} & no \\
& {$\left[X_{6}, X_{1}\right]=2 X_{1},\left[X_{6}, X_{2}\right]=X_{2}$,} & \\
& {$\left[X_{6}, X_{3}\right]=X_{3},\left[X_{6}, X_{4}\right]=-4 X_{4}$} & \\
\hline
\end{tabular}

In the introduction of [43], Mubarakzjanov quotes his own result that a sixdimensional solvable Lie algebra with three-dimensional nilradical is decomposable. Therefore, by Proposition 3.5, we have listed all unimodular indecomposable solvable Lie algebras of dimension six.

The first Betti numbers of the six-dimensional unimodular indecomposable Lie algebras are listed in Tables 34-36. The word "always" means that the certain value arises independent of the parameters on which the Lie algebra depends, but we suppose that the parameters are chosen such that Lie algebra is unimodular. The word "otherwise" in the tables means that this value arises for all parameters such that the Lie algebra is unimodular and the parameters are not mentioned in another column of the Lie algebra's row.

Table 34: $b_{1}\left(\mathfrak{g}_{6 . i}\right)$ for $\mathfrak{g}_{6 . i}$ unimodular

\begin{tabular}{|c|c|c|c|}
\hline \hline$i$ & $b_{1}=1$ & $b_{1}=2$ & $b_{1}=3$ \\
\hline \hline 1 & always & - & - \\
\hline 2 & $a \neq 0$ & $a=0$ & - \\
\hline 3 & $d \neq-1$ & $d=-1$ & - \\
\hline 4 & always & - & - \\
\hline 6 & $a, b \neq 0$ & $a=-\frac{1}{2} \wedge b=0$ & - \\
\hline 7 & always & - & - \\
\hline 8 & always & - & - \\
\hline 9 & $b \neq 0$ & $b=0$ & - \\
\hline 10 & $a \neq 0$ & $a=0$ & - \\
\hline 11 & always & - & - \\
\hline 12 & always & - & - \\
\hline 13 & $b \neq 0 \wedge h \neq 0$ & otherwise & $a=-\frac{1}{2} \wedge b=h=0$ \\
\hline 14 & otherwise & $a=-\frac{1}{3} \wedge b=0$ & - \\
\hline 15 & always & - & - \\
\hline 17 & - & always & - \\
\hline 18 & $a \neq 0$ & $a=0$ & - \\
\hline 19 & always & - & - \\
\hline 20 & - & always & - \\
\hline
\end{tabular}


Table 35: $b_{1}\left(\mathfrak{g}_{6 . i}\right)$ for $\mathfrak{g}_{6 . i}$ unimodular (continued)

\begin{tabular}{|c|c|c|c|c|c|}
\hline$i$ & $b_{1}=1$ & $b_{1}=2$ & $b_{1}=3$ & $b_{1}=4$ & $b_{1}=5$ \\
\hline 21 & $a \neq 0$ & $\bar{a} a=0$ & - & - & - \\
\hline 22 & always & - & - & - & - \\
\hline 23 & $a \neq 0$ & - & $a=0$ & - & - \\
\hline 25 & $b \notin\{-1,0\}$ & $b \in\{-1,0\}$ & - & - & - \\
\hline 26 & - & always & - & - & - \\
\hline 27 & always & - & - & - & - \\
\hline 28 & always & - & - & - & - \\
\hline 29 & $b \neq 0$ & - & $b=0$ & - & - \\
\hline 32 & $h \notin\{-2 a,-6 a\}$ & otherwise & - & - & - \\
\hline 33 & $a \neq 0$ & - & $a=0$ & - & - \\
\hline 34 & $a \neq 0$ & - & $a=0$ & - & - \\
\hline 35 & $a, b \neq 0$ & otherwise & - & - & - \\
\hline 36 & $a \neq 0$ & $a=0$ & - & - & - \\
\hline 37 & always & - & - & - & - \\
\hline 38 & always & - & - & - & - \\
\hline 39 & $h \neq 0$ & $h=0$ & - & - & - \\
\hline 40 & always & - & - & - & - \\
\hline 41 & always & - & - & - & - \\
\hline 42 & always & - & - & - & - \\
\hline 44 & always & - & - & - & - \\
\hline 47 & - & always & - & - & - \\
\hline 54 & $l \notin\left\{-2,-1,-\frac{1}{2}\right\}$ & $l \in\left\{-2,-1,-\frac{1}{2}\right\}$ & - & - & - \\
\hline 55 & always & - & - & - & - \\
\hline 56 & always & - & - & - & - \\
\hline 57 & always & - & - & - & - \\
\hline 61 & always & - & - & - & - \\
\hline 63 & - & always & - & - & - \\
\hline 65 & $l \neq 0$ & - & $l=0$ & - & - \\
\hline 70 & $p \neq 0$ & $p=0$ & - & - & - \\
\hline 71 & always & - & - & - & - \\
\hline 76 & always & - & - & - & - \\
\hline 78 & always & - & - & - & - \\
\hline 83 & $l \neq 0$ & - & - & $l=0$ & - \\
\hline 84 & - & always & - & - & - \\
\hline 88 & $\mu_{0} \neq 0 \vee \nu_{0} \neq 0$ & - & - & - & $\mu_{0}=\nu_{0}=0$ \\
\hline 89 & $\nu_{0} \neq 0 \wedge s \neq 0$ & - & otherwise & - & $\nu_{0}=s=0$ \\
\hline 90 & $\nu_{0} \neq 0$ & - & $\nu_{0}=0$ & - & - \\
\hline
\end{tabular}


Table 36: $b_{1}\left(\mathfrak{g}_{6 . i}\right)$ for $\mathfrak{g}_{6 . i}$ unimodular (continued)

\begin{tabular}{|c|c|c|c|c|c|}
\hline \hline$i$ & $b_{1}=1$ & $b_{1}=2$ & $b_{1}=3$ & $b_{1}=4$ & $b_{1}=5$ \\
\hline \hline 91 & always & - & - & - & - \\
\hline 92 & $\mu_{0} \neq 0 \wedge \nu_{0} \neq 0$ & - & otherwise & - & $\mu_{0}=\nu_{0}=0$ \\
\hline $92 *$ & always & - & - & - & - \\
\hline 93 & $\nu_{0} \neq 0$ & - & $\nu_{0}=0$ & - & - \\
\hline 94 & always & - & - & - & - \\
\hline 101 & - & always & - & - & - \\
\hline 102 & - & always & - & - & - \\
\hline 105 & - & always & - & - & - \\
\hline 107 & - & always & - & - & - \\
\hline 113 & - & always & - & - & - \\
\hline 114 & - & always & - & - & - \\
\hline 115 & - & always & - & - & - \\
\hline 116 & - & always & - & - & - \\
\hline 118 & - & always & - & - & - \\
\hline 120 & - & always & - & - & - \\
\hline 125 & - & always & - & - & - \\
\hline 129 & - & always & - & - & - \\
\hline 135 & - & always & - & - & - \\
\hline
\end{tabular}




\section{B Integer Polynomials}

In this article, we often try to use necessary conditions for a matrix to be conjugated to an integer matrix. We state briefly the used results. Vice versa, we sometimes want to find integer matrices with given minimal polynomial. We also present a few constructions.

Let be $n \in \mathbb{N}_{+}, \mathbb{K}$ a field and $A \in \mathrm{M}(n, n ; \mathbb{K})$. The characteristic polynomial of $A$ is the monic polynomial

$$
P_{A}(X):=\operatorname{det}(X \text { id }-A) \in \mathbb{K}[X],
$$

and the minimal polynomial $m_{A}(X)$ is the unique monic divisor of lowest degree of $P_{A}(X)$ in $\mathbb{K}[X]$ such that $m_{A}(A)=0$. (Note, by the theorem of Cayley-Hamilton, one has $P_{A}(A)=0$.)

If two matrices are conjugated, then they have the same characteristic resp. minimal polynomials.

$\lambda \in \overline{\mathbb{K}}$ is called root of $A$ if $\lambda$ is a root of the characteristic polynomial, considered as polynomial in $\overline{\mathbb{K}}[X]$, where $\overline{\mathbb{K}}$ denotes the algebraic closure of $\mathbb{K}$.

The next proposition follows directly from [36, Corollaries XIV.2.2, XIV.2.3].

Proposition B.1. Let $n \in \mathbb{N}_{+}$. If $A \in \mathrm{M}(n, n ; \mathbb{C})$ and $B \in \mathrm{M}(n, n ; \mathbb{Q})$ are conjugated via an element of $\mathrm{GL}(n, \mathbb{C})$, then holds $P_{A}(X)=P_{B}(X) \in \mathbb{Q}[X]$, $m_{A}(X)=m_{B}(X) \in \mathbb{Q}[X]$ and $m_{A}(X)$ divides $P_{A}(X)$ in $\mathbb{Q}[X]$.

Proposition B.2. If $P(X) \in \mathbb{Z}[X], m(X) \in \mathbb{Q}[X]$ are monic polynomials and $m(X)$ divides $P(X)$ in $\mathbb{Q}[X]$, then holds $m(X) \in \mathbb{Z}[X]$.

Proof. Let $P(X), m(X)$ be as in the proposition and $f(X) \in \mathbb{Q}[X]$ nonconstant with $P(X)=f(X) m(X)$. There exist $k, l \in \mathbb{Z} \backslash\{0\}$ such that

$$
k f(X)=\sum_{i} a_{i} X^{i}, \operatorname{lm}(X)=\sum_{j} b_{j} X^{j} \in \mathbb{Z}[X]
$$

are primitive. (An integer polynomial is called primitive if its coefficients are relatively prime.) We have

$$
k l P(X)=\left(\sum_{i} a_{i} X^{i}\right)\left(\sum_{j} b_{j} X^{j}\right)
$$

and claim $k l= \pm 1$.

Otherwise, there is a prime $p \in \mathbb{N}$ that divides $k l$. Since the coefficients of $k f(X)$ resp. $\operatorname{lm}(X)$ are relatively prime, there are minimal $i_{0}, j_{0} \in \mathbb{N}$ such that $p$ does not divide $a_{i_{0}}$ resp. $b_{j_{0}}$.

The coefficient of $X^{i_{0}+j_{0}}$ of $k l f(X) m(X)$ is

$$
a_{i_{0}} b_{j_{0}}+a_{i_{0}-1} b_{j_{0}+1}+a_{i_{0}+1} b_{j_{0}-1}+\ldots
$$


and $p$ divides each summand except the first. But since $p \mid k l, p$ divides the whole sum. This is a contradiction.

Theorem B.3. Let $n \in \mathbb{N}_{+}$and $A \in \mathrm{M}(n, n ; \mathbb{C})$ be conjugated to an integer matrix. Then holds $P_{A}(X), m_{A}(X) \in \mathbb{Z}[X]$.

Proof. This follows from the preceding two propositions.

Lemma B.4 ([30, Lemma 2.2]). Let $P(X)=X^{3}-k X^{2}+l X-1 \in \mathbb{Z}[X]$.

Then $P$ has a double root $X_{0} \in \mathbb{R}$ if and only if $X_{0}=1$ or $X_{0}=-1$ for which $P(X)=X^{3}-3 X^{2}+3 X-1$ or $P(X)=X^{3}+X^{2}-X-1$ respectively.

Proposition B.5 ([28, Proposition 5]). Let $\lambda_{i} \in \mathbb{R}_{+}$with $\lambda_{i}+\frac{1}{\lambda_{i}}=m_{i} \in \mathbb{N}_{+}$and $m_{i}>2$ for $i \in\{1,2\}$.

Then there exists no element in $\mathrm{SL}(3, \mathbb{Z})$ with roots $\lambda_{1}, \lambda_{2}, \frac{1}{\lambda_{1} \lambda_{2}}$.

Proposition B.6. Let $P(X)=X^{4}-m X^{3}+p X^{2}-n X+1 \in \mathbb{Z}[X]$.

Then $P$ has a root with multiplicity $>1$ if and only if the zero set of $P$ equals $\left\{1,1, a, a^{-1}\right\},\left\{-1,-1, a, a^{-1}\right\},\left\{a, a^{-1}, a, a^{-1}\right\}$ or $\left\{a,-a^{-1}, a,-a^{-1}\right\}$ for fixed $a \in \mathbb{C}$.

Proof. The most part of the proof was done by Harshavardhan in the proof of [28, Propositon 2].

We set $S:=m^{2}+n^{2}$ and $T:=m n$ and get the discriminant $D$ of $P(X)$ as

$$
\begin{aligned}
D= & 16 p^{4}-4 S p^{3}+\left(T^{2}-80 T-128\right) p^{2}+18 S(T+8) p \\
& +256-192 T+48 T^{2}-4 T^{3}-27 S^{2} .
\end{aligned}
$$

Note that $P(X)$ has a root of multiplicity $>1$ if and only if $D=0$. Solving $D=0$ for $S$, we see

$$
S=-\frac{2}{27} p^{3}+\frac{1}{3} p T+\frac{8}{3} p \pm \frac{2}{27} \sqrt{\left(p^{2}-3 T+12\right)^{3}}
$$

and since $S$ and $T$ are integers, there is $q \in \mathbb{Z}$ with

$$
p^{2}-3 T+12=q^{2}
$$

which implies

$$
\begin{aligned}
S & =4 p+\frac{1}{27}\left(p^{3}-3 p q^{2} \pm 2 q^{3}\right) \\
T & =\frac{1}{3}\left(p^{2}-q^{2}+12\right) .
\end{aligned}
$$

We first consider the plus sign in equation (18). Then one has

$$
(m+n)^{2}=S+2 T=\frac{1}{27}(p+2 q+6)(p-q+6)^{2},
$$




$$
(m-n)^{2}=S-2 T=\frac{1}{27}(p+2 q-6)(p-q-6)^{2},
$$

and this implies the existence of $k_{i}, l_{i} \in \mathbb{N}, i=1,2$, such that

$$
\begin{aligned}
3 k_{1}^{2} & =(p+2 q+6) k_{2}^{2}, \\
3 l_{1}^{2} & =(p+2 q-6) l_{2}^{2} .
\end{aligned}
$$

We shall show: $|m|=|n|$

[If $l_{2}=0$, the claim is proved. Therefore, we can assume $l_{2} \neq 0$.

Case 1: $k_{2}=0$

Then holds $k_{1}=0$ and this means $S+2 T=0$, i.e. $(m+n)^{2}=0$, so we have $m=-n$.

Case 2: $k_{2} \neq 0$

We write $k:=\frac{k_{1}}{k_{2}}$ and $l:=\frac{l_{1}}{l_{2}}$. Then holds

$$
\begin{aligned}
3 k^{2} & =p+2 q+6 \in \mathbb{Z}, \\
3 l^{2} & =p+2 q-6 \in \mathbb{Z},
\end{aligned}
$$

and $3\left(k^{2}-l^{2}\right)=12$. Therefore, we have $k^{2}-l^{2}=4$, so $k^{2}=4, l^{2}=0$, i.e. $l_{1}=0$, $S-2 T=0$ and $m=n$. ]

Now, consider the minus sign in equation (18). Then one has

$$
\begin{aligned}
& (m+n)^{2}=S+2 T=\frac{1}{27}(p-2 q+6)(p+q+6)^{2}, \\
& (m-n)^{2}=S-2 T=\frac{1}{27}(p-2 q-6)(p+q-6)^{2},
\end{aligned}
$$

and shows analogously as above $|m|=|n|$.

We have shown: If $P(X)$ has a multiple root, then holds $m= \pm n$.

If $m=n$, then one calculates the solutions of $D=0$ in (17) as the following

(i) $p=-2+2 m$,

(ii) $p=-2-2 m$,

(iii) $p=2+\frac{m^{2}}{4}$,

and if $m=-n$, then the real solution of $D=0$ in (17) is

(iv) $p=-2+\frac{m^{2}}{4}$.

Moreover, a short computation yields the zero set of $P(X)$ in the cases (i) (iv) as $\left\{1,1, a, a^{-1}\right\},\left\{-1,-1, a, a^{-1}\right\},\left\{a, a^{-1}, a, a^{-1}\right\},\left\{a,-a^{-1}, a,-a^{-1}\right\}$, respectively. 
Proposition B.7 ([1, Proposition 4.4.14]). Let $\mathbb{K}$ be a field and

$$
m(X)=X^{n}+a_{n-1} X^{n-1}+\ldots+a_{1} X^{1}+a_{0} \in \mathbb{K}[X]
$$

a monic polynomial. Then $\left(\begin{array}{cccccc}0 & 0 & \ldots & 0 & 0 & -a_{0} \\ 1 & 0 & \ldots & 0 & 0 & -a_{1} \\ 0 & 1 & \ldots & 0 & 0 & -a_{2} \\ \vdots & \vdots & \ddots & \vdots & \vdots & \vdots \\ 0 & 0 & \ldots & 1 & 0 & -a_{n-2} \\ 0 & 0 & \ldots & 0 & 1 & -a_{n-1}\end{array}\right)$ has minimal polynomial $m(X)$.

If one is willing to construct an integer matrix with given characteristic and minimal polynomial, one always can chose any matrix $M$ which has the desired polynomials and try to find an invertible matrix $T$ such that $T^{-1} M T$ has integer entries. Of course, this can be difficult. In the case of $4 \times 4$ - matrices we have the following easy construction.

Proposition B.8 ([28, Section 2.3.1]).

(i) Let integers $m, n, p \in \mathbb{Z}$ be given.

Choose $m_{1}, \ldots, m_{4} \in \mathbb{Z}$ such that $\sum_{i=1}^{4} m_{i}=m$ and set

$$
\begin{aligned}
a:= & -m_{1}^{2} p+m_{1}^{3} m_{2}+m_{1}^{3} m_{3}+m_{1}^{3} m_{4}+m_{1} n-1, \\
b:= & \left(-m_{2}-m_{1}\right) p+m_{1} m_{2}^{2}+m_{1} m_{2} m_{3}+m_{1} m_{2} m_{4}+m_{2}^{2} m_{3}+m_{2}^{2} m_{4} \\
& +m_{1}^{2} m_{2}+m_{1}^{2} m_{3}+m_{1}^{2} m_{4}+n, \\
c:= & m_{1} m_{2}+m_{1} m_{3}+m_{1} m_{4}+m_{2} m_{3}+m_{2} m_{4}+m_{3} m_{4}-p .
\end{aligned}
$$

Then the matrix $\left(\begin{array}{cccc}m_{1} & 0 & 0 & a \\ 1 & m_{2} & 0 & b \\ 0 & 1 & m_{3} & c \\ 0 & 0 & 1 & m_{4}\end{array}\right)$ has $X^{4}-m X^{3}+p X^{2}-n X+1$ as characteristic polynomial.

(ii) Let $m \in 2 \mathbb{Z}$ be an even integer. Then the matrix $\left(\begin{array}{cccc}\frac{m}{2} & 0 & -1 & 0 \\ 0 & \frac{m}{2} & 0 & -1 \\ 1 & 0 & 0 & 0 \\ 0 & 1 & 0 & 0\end{array}\right)$ has the characteristic polynomial $\left(X^{2}-\frac{m}{2} X+1\right)^{2}$, and $\left(X^{2}-\frac{m}{2} X+1\right)$ as minimal polynomial.

Acknowledgement. The results presented in this paper were obtained in my dissertation under the supervision of Prof. H. Geiges. I wish to express my sincerest gratitude for his support during the last four years. 


\section{References}

[1] W. A. Adkins, St. H. Weintraub: Algebra, Springer (1992).

[2] L. Auslander: An exposition of the structure of solvmanifolds - part I, Bull. Amer. Math. Soc. 79 (1973), no. 2, 227-261.

[3] L. Auslander, L. Green, F. Hahn: Flows on Homogeneous Spaces, Princeton University Press (1963).

[4] Ch. Benson, C. S. Gordon: Kähler and symplectic structures on nilmanifolds, Topology 27 (1988), no. 4, 513-518.

[5] Ch. Benson, C. S. Gordon: Kähler structures on compact solvmanifolds, Proc. Amer. Math. Soc. 108 (1990), no. 4, 971-980.

[6] Ch. Bock: Geography of non-formal symplectic and contact manifolds, arXiv:0812.1447.

[7] S. Bosch: Lineare Algebra, Springer (2006).

[8] K. S. Brown: Cohomology of Groups, Springer (1982).

[9] R. Campoamor-Stursberg: Symplectic forms on six dimensional real solvable Lie algebras, arXiv:math.DG/0507499.

[10] R. Campoamor-Stursberg: Some remarks concerning the invariants of rank one solvable real Lie algebras, Algebra Colloq. 12 (2005), no. 3, 497-518.

[11] B.-Y. Chu: Symplectic homogeneous spaces, Trans. Amer. Math. Soc. 197 (1974), 145-159.

[12] L. Corwin, F. P. Greenleaf: Representations of Nilpotent Lie Groups and Their Applications, Cambridge University Press (1990).

[13] K. Dekimpe: Semi-simple splittings for solvable Lie groups and polynomial structures, Forum Math. 12 (2000), no. 1, 77-96.

[14] P. Deligne, P. Griffiths, J. Morgan, D. Sullivan: Real homotopy theory of Kähler manifolds, Invent. Math. 29 (1975), no. 3, 245-274.

[15] A. Diatta: Left invariant contact structures on Lie groups, Differential Geom. Appl. 26 (2008), no. 5, 544-552.

[16] J. Dixmier: Sur les algèbres dérivées d'une algèbre de Lie, Proc. Cambride Philos. Soc. 51 (1955), 541-544. 
[17] J. Dixmier: L'application exponentielle dans les groupes de Lie résolubles, Bull. Soc. Math. France 85 (1957), 113-121.

[18] N. Dungey, A. F. M. ter Elst, D. W. Robinson: Analysis on Lie Groups with Polynomial Growth, Birkhäuser (2003).

[19] M. Fernández, A. Gray: Compact symplectic solvmanifolds not admitting complex structures, Geom. Dedicata 34 (1990), no. 3, 295-299.

[20] M. Fernández, M. de León, M. Saralegui: A six-dimensional compact symplectic solvmanifold without Kähler structures, Osaka J. Math. 33 (1996), no. 1, 19-35.

[21] M. Fernández, V. Muñoz: Formality of Donaldson submanifolds, Math. Z. 250 (2005), no. 1, 149-175.

[22] M. Fernández, V. Muñoz: The geography of non-formal manifolds in O. Kowalski, E. Musso, D. Perrone: Complex, Contact and Symmetric Manifolds, 121-129, Prog. Math. vol. 234, Birkhäuser (2005).

[23] S. Fukuhara, K. Sakamoto: Classification of $T^{2}$-bundles over $T^{2}$, Tokyo J. Math. 6 (1983), no. 2, 311-327.

[24] H. Geiges: Symplectic structures on $T^{2}$-bundles over $T^{2}$, Duke Math. J. 67 (1992), no. 3, 539-555.

[25] H. Geiges: Symplectic manifolds with disconnected boundary of contact type, Internat. Math. Res. Notices (1994), no. 1, 23-30.

[26] V. V. Gorbatsevich: Symplectic structures and cohomologies on some solvmanifolds, Sibiran Math. J. 44 (2003), no. 2, 260-274.

[27] P. Griffiths, J. Harris: Principles of Algebraic Geometry, Wiley (1994).

[28] R. Harshavardhan: Geometric structures of Lie type on 5-manifolds, Ph.D. Thesis, Cambridge University (1996).

[29] K. Hasegawa: Minimal models of nilmanifolds, Proc. Amer. Math. Soc. 106 (1989), no. 1, 65-71.

[30] K. Hasegawa: Four-dimensional compact solvmanifolds with and without complex analytic structures, arXiv:math.CV/0401413.

[31] K. Hasegawa: Complex and Kähler structures on compact solvmanifolds, J. Symplectic Geom. 3 (2005), no. 4, 749-767.

[32] K. Hasegawa: A note on compact solvmanifolds with Kähler structure, Osaka J. Math. 43 (2006), no. 1, 131-135. 
[33] A. Hattori: Spectral sequence in the de Rham cohomology of fibre bundles, J. Fac. Sci. Univ. Tokyo Sect. I 8 (1960), 289-331.

[34] R. Ibáñez, Y. Rudiak, A. Tralle, L. Ugarte: On certain geometric and homotopy properties of closed symplectic manifolds, Topology Appl. 127 (2003), no. $1-2,33-45$.

[35] J. Kȩdra, Y. Rudyak, A. Tralle: Symplectically aspherical manifolds, J. Fixed Point Theory Appl. 3 (2008), no. 1, 1-21.

[36] S. Lang: Algebra, 3. ed., Addison-Wesley (1993).

[37] R. Lutz: Sur la géométrie des structures de contact invariantes, Ann. Inst. Fourier (Grenoble), 29 (1979), no. 1, xvii, 283-306.

[38] J. Milnor: Curvature of left invariant metrics on Lie groups, Advances in Math. 21 (1976), no. 3, 293-329.

[39] V. V. Morozov: Classifikaziya nilpotentnych algebrach Lie shestovo poryadka, Izv. Vyš̆. Učebn. Zaved. Mathematika 5 (1958), no. 4, 161-171.

[40] G. D. Mostow: Factor spaces of solvable groups, Ann. of Math. (2) 60 (1954), $1-27$.

[41] G. M. Mubarakzjanov: O rasreshimych algebrach Lie, Izv. Vyš̆. Učebn. Zaved. Mathematika 32 (1963), no. 1, 114-123.

[42] G. M. Mubarakzjanov: Classifkaziya vehestvennych structur algebrach Lie pyatovo poryadko, Izv. Vyš̆. Učebn. Zaved. Mathematika 34 (1963), no. 3, 99-106.

[43] G. M. Mubarakzjanov: Classifkaziya rasreshimych structur algebrach Lie shestovo poryadka a odnim nenilpoentnym bazisnym elementom, Izv. Vyš̆. Učebn. Zaved. Mathematika 35 (1963), no. 4, 104-116.

[44] I. Nakamura: Complex parallelisable manifolds and their small deformations, J. Differential Geometry 10 (1975), 85-112.

[45] A. Newlander, L. Nirenberg: Complex analytic coordinates in almost complex manifolds, Ann. of Math. (2) 65 (1957), 391-404.

[46] K. Nomizu: On the cohomology of homogeneous spaces of nilpotent Lie Groups, Ann. of Math. (2) 59 (1954), 531-538.

[47] T. Nôno: On the singularity of general linear groups, J. Sci. Hiroshima Univ. Ser. A 20 (1956/1957), 115-123. 
[48] J. Oprea, A. Tralle: Symplectic Manifolds with no Kähler Structure, Lecture Notes in Math. 1661, Springer (1997).

[49] A. L. Onishchik, E. B. Vinberg: Lie Groups and Lie Algebras III, Springer (1994).

[50] M. S. Raghunathan: Discrete Subgroups of Lie Groups, Springer (1972).

[51] M. Saitô: Sur certaines groupes de Lie résolubles - parties I et II, Sci. Papers Coll. Gen. Ed. Univ. Tokyo 7 (1957), 1-11, 157-168.

[52] S. M. Salamon: Complex structures on nilpotent Lie algebras, J. Pure Appl. Algebra 157 (2001), no. 2-3, 311-333.

[53] H. Sawai, T. Yamada: Lattices on Benson-Gordon type solvable Lie groups, Topology Appl. 149 (2005), no. 1-3, 85-95.

[54] N. Steenrod: The Topology of Fibre Bundles, Princeton University Press (1951).

[55] D. Sullivan: Infinitesimal computations in topology, Inst. Hautes Études Sci. Publ. Math. 47 (1977), 269-331.

[56] W. P. Thurston: Some simple examples of compact symplectic manifolds, Proc. Amer. Math. Soc. 55 (1976), no. 2, 467-468.

[57] A. Tralle: On solvable Lie groups without lattices in M. Fernández, J. A. Wolf: Global Differential Geometry, 437-441, Contemp. Math. 288, Amer. Math. Soc. (2001).

[58] P. Turkowski: Solvable Lie algebras of dimension six, J. Math. Phys. 31 (1990), no. 6, 1344-1350.

[59] V. S. Vararadarajan: Lie Groups, Lie Algebras, and Their Representations, Springer (1984).

[60] F. W. Warner: Foundations of Differentiable Manifolds and Lie Groups, Glenview (1971).

[61] Ch. A. Weibel: An Introduction to Homological Algebra, Cambridge University Press (1997).

[62] T. Yamada: A pseudo-Kähler structure on a nontoral compact complex parallelizable solvmanifold, Geom. Dedicata 112 (2005), 115-122.

Christoph Bock, Universität ZU KÖln, Mathematisches Institut, Weyertal 86-90, 50931 Köln, Germany

e-mail: bock@math.uni-koeln.de 